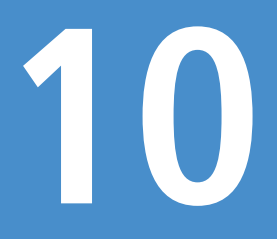

\title{
Detection and Attribution of Climate Change: from Global to Regional
}

\author{
Coordinating Lead Authors: \\ Nathaniel L. Bindoff (Australia), Peter A. Stott (UK)
}

\section{Lead Authors:}

Krishna Mirle AchutaRao (India), Myles R. Allen (UK), Nathan Gillett (Canada), David Gutzler (USA), Kabumbwe Hansingo (Zambia), Gabriele Hegerl (UK/Germany), Yongyun Hu (China), Suman Jain (Zambia), Igor I. Mokhov (Russian Federation), James Overland (USA), Judith Perlwitz (USA), Rachid Sebbari (Morocco), Xuebin Zhang (Canada)

\section{Contributing Authors:}

Magne Aldrin (Norway), Beena Balan Sarojini (UK/India), Jürg Beer (Switzerland), Olivier Boucher (France), Pascale Braconnot (France), Oliver Browne (UK), Ping Chang (USA), Nikolaos Christidis (UK), Tim DelSole (USA), Catia M. Domingues (Australia/Brazil), Paul J. Durack (USA/ Australia), Alexey Eliseev (Russian Federation), Kerry Emanuel (USA), Graham Feingold (USA), Chris Forest (USA), Jesus Fidel González Rouco (Spain), Hugues Goosse (Belgium), Lesley Gray (UK), Jonathan Gregory (UK), Isaac Held (USA), Greg Holland (USA), Jara Imbers Quintana (UK), William Ingram (UK), Johann Jungclaus (Germany), Georg Kaser (Austria), Veli-Matti Kerminen (Finland), Thomas Knutson (USA), Reto Knutti (Switzerland), James Kossin (USA), Mike Lockwood (UK), Ulrike Lohmann (Switzerland), Fraser Lott (UK), Jian Lu (USA/Canada), Irina Mahlstein (Switzerland), Valérie Masson-Delmotte (France), Damon Matthews (Canada), Gerald Meehl (USA), Blanca Mendoza (Mexico), Viviane Vasconcellos de Menezes (Australia/ Brazil), Seung-Ki Min (Republic of Korea), Daniel Mitchell (UK), Thomas Mölg (Germany/ Austria), Simone Morak (UK), Timothy Osborn (UK), Alexander Otto (UK), Friederike Otto (UK), David Pierce (USA), Debbie Polson (UK), Aurélien Ribes (France), Joeri Rogelj (Switzerland/ Belgium), Andrew Schurer (UK), Vladimir Semenov (Russian Federation), Drew Shindell (USA), Dmitry Smirnov (Russian Federation), Peter W. Thorne (USA/Norway/UK), Muyin Wang (USA), Martin Wild (Switzerland), Rong Zhang (USA)

\section{Review Editors:}

Judit Bartholy (Hungary), Robert Vautard (France), Tetsuzo Yasunari (Japan)

This chapter should be cited as:

Bindoff, N.L., P.A. Stott, K.M. AchutaRao, M.R. Allen, N. Gillett, D. Gutzler, K. Hansingo, G. Hegerl, Y. Hu, S. Jain, I.I. Mokhov, J. Overland, J. Perlwitz, R. Sebbari and X. Zhang, 2013: Detection and Attribution of Climate Change: from Global to Regional. In: Climate Change 2013: The Physical Science Basis. Contribution of Working Group I to the Fifth Assessment Report of the Intergovernmental Panel on Climate Change [Stocker, T.F., D. Qin, G.-K. Plattner, M. Tignor, S.K. Allen, J. Boschung, A. Nauels, Y. Xia, V. Bex and P.M. Midgley (eds.)]. Cambridge University Press, Cambridge, United Kingdom and New York, NY, USA. 


\section{Table of Contents}

Executive Summary

10.1 Introduction

872

\subsection{Evaluation of Detection and Attribution} Methodologies

10.2.1 The Context of Detection and Attribution 872

10.2.2 Time Series Methods, Causality and Separating Signal from Noise 874

Box 10.1: How Attribution Studies Work 875

10.2.3 Methods Based on General Circulation Models and Optimal Fingerprinting

10.2.4 Single-Step and Multi-Step Attribution and the Role of the Null Hypothesis.

10.3 Atmosphere and Surface 878

10.3.1 Temperature... 878

Box 10.2: The Sun's Influence on the Earth's Climate ........... 885

10.3.2 Water Cycle 895

10.3.3

Atmospheric Circulation and Patterns of Variability

10.4 Changes in Ocean Properties 901

10.4

Ocean Temperature and Heat Content 901

10.4.2 Ocean Salinity and Freshwater Fluxes ....................... 903

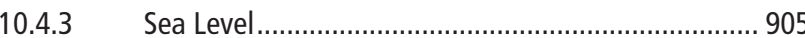

10.4.4 Oxygen and Ocean Acidity ....................................... 905

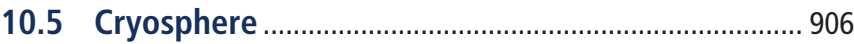

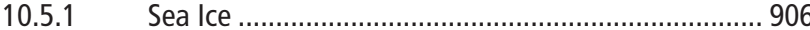

10.5.2 Ice Sheets, Ice Shelves and Glaciers ......................... 909

10.5.3 Snow Cover.............................................................. 910

10.6 Extremes 910

10.6.1 Attribution of Changes in Frequency/Occurrence and Intensity of Extremes.. 910

10.6.2 Attribution of Weather and Climate Events.... 914
10.7 Multi-century to Millennia Perspective 917

10.7.1 Causes of Change in Large-Scale Temperature over the Past Millennium ..................................................... 917

10.7.2 Changes of Past Regional Temperature ..................... 919

10.7.3 Summary: Lessons from the Past.............................. 919

10.8 Implications for Climate System Properties and Projections ............................................................. 920

10.8.1 Transient Climate Response ..................................... 920

10.8.2 Constraints on Long-Term Climate Change and the Equilibrium Climate Sensitivity................................. 921

10.8.3 Consequences for Aerosol Forcing and Ocean Heat Uptake ............................................................. 926

10.8.4 Earth System Properties ......................................... 926

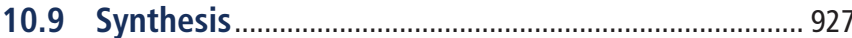

10.9.1 Multi-variable Approaches ...................................... 927

10.9.2 Whole Climate System ............................................ 927

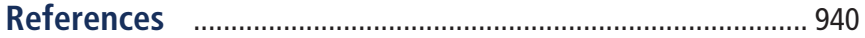

\section{Frequently Asked Questions}

FAQ 10.1 Climate Is Always Changing. How Do We Determine the Causes of Observed Changes?

FAQ 10.2 When Will Human Influences on Climate Become Obvious on Local Scales? 928

\section{Supplementary Material}

Supplementary Material is available in online versions of the report. 


\section{Executive Summary}

\section{Atmospheric Temperatures}

More than half of the observed increase in global mean surface temperature (GMST) from 1951 to 2010 is very likely due to the observed anthropogenic increase in greenhouse gas (GHG) concentrations. The consistency of observed and modeled changes across the climate system, including warming of the atmosphere and ocean, sea level rise, ocean acidification and changes in the water cycle, the cryosphere and climate extremes points to a large-scale warming resulting primarily from anthropogenic increases in GHG concentrations. Solar forcing is the only known natural forcing acting to warm the climate over this period but it has increased much less than GHG forcing, and the observed pattern of long-term tropospheric warming and stratospheric cooling is not consistent with the expected response to solar irradiance variations. The Atlantic Multi-decadal Oscillation (AMO) could be a confounding influence but studies that find a significant role for the AMO show that this does not project strongly onto 1951-2010 temperature trends. \{10.3.1, Table 10.1\}

It is extremely likely that human activities caused more than half of the observed increase in GMST from 1951 to 2010. This assessment is supported by robust evidence from multiple studies using different methods. Observational uncertainty has been explored much more thoroughly than previously and the assessment now considers observations from the first decade of the 21st century and simulations from a new generation of climate models whose ability to simulate historical climate has improved in many respects relative to the previous generation of models considered in AR4. Uncertainties in forcings and in climate models' temperature responses to individual forcings and difficulty in distinguishing the patterns of temperature response due to GHGs and other anthropogenic forcings prevent a more precise quantification of the temperature changes attributable to GHGs. \{9.4.1, 9.5.3, 10.3.1, Figure 10.5, Table 10.1\}

GHGs contributed a global mean surface warming likely to be between $0.5^{\circ} \mathrm{C}$ and $1.3^{\circ} \mathrm{C}$ over the period $1951-2010$, with the contributions from other anthropogenic forcings likely to be between $-0.6^{\circ} \mathrm{C}$ and $0.1^{\circ} \mathrm{C}$, from natural forcings likely to be between $-0.1^{\circ} \mathrm{C}$ and $0.1^{\circ} \mathrm{C}$, and from internal variability likely to be between $-0.1^{\circ} \mathrm{C}$ and $0.1^{\circ} \mathrm{C}$. Together these assessed contributions are consistent with the observed warming of approximately $0.6^{\circ} \mathrm{C}$ over this period. $\{10.3 .1$, Figure 10.5$\}$

It is virtually certain that internal variability alone cannot account for the observed global warming since 1951. The observed global-scale warming since 1951 is large compared to climate model estimates of internal variability on 60 -year time scales. The
Northern Hemisphere $(\mathrm{NH})$ warming over the same period is far outside the range of any similar length trends in residuals from reconstructions of the past millennium. The spatial pattern of observed warming differs from those associated with internal variability. The model-based simulations of internal variability are assessed to be adequate to make this assessment. $\{9.5 .3,10.3 .1,10.7 .5$, Table 10.1\}

It is likely that anthropogenic forcings, dominated by GHGs, have contributed to the warming of the troposphere since 1961 and very likely that anthropogenic forcings, dominated by the depletion of the ozone layer due to ozone-depleting substances, have contributed to the cooling of the lower stratosphere since 1979. Observational uncertainties in estimates of tropospheric temperatures have now been assessed more thoroughly than at the time of AR4. The structure of stratospheric temperature trends and multi-year to decadal variations are well represented by models and physical understanding is consistent with the observed and modelled evolution of stratospheric temperatures. Uncertainties in radiosonde and satellite records make assessment of causes of observed trends in the upper troposphere less confident than an assessment of the overall atmospheric temperature changes. $\{2.4 .4,9.4 .1,10.3 .1$, Table 10.1 $\}$

Further evidence has accumulated of the detection and attribution of anthropogenic influence on temperature change in different parts of the world. Over every continental region, except Antarctica, it is likely that anthropogenic influence has made a substantial contribution to surface temperature increases since the mid20th century. The robust detection of human influence on continental scales is consistent with the global attribution of widespread warming over land to human influence. It is likely that there has been an anthropogenic contribution to the very substantial Arctic warming over the past 50 years. For Antarctica large observational uncertainties result in low confidence ${ }^{2}$ that anthropogenic influence has contributed to the observed warming averaged over available stations. Anthropogenic influence has likely contributed to temperature change in many sub-continental regions. $\{2.4 .1,10.3 .1$, Table 10.1\}

Robustness of detection and attribution of global-scale warming is subject to models correctly simulating internal variability. Although estimates of multi-decadal internal variability of GMST need to be obtained indirectly from the observational record because the observed record contains the effects of external forcings (meaning the combination of natural and anthropogenic forcings), the standard deviation of internal variability would have to be underestimated in climate models by a factor of at least three to account for the observed warming in the absence of anthropogenic influence. Comparison with observations provides no indication of such a large difference between climate models and observations. \{9.5.3, Figures 9.33, 10.2, 10.3.1, Table 10.1\}

1 In this Report, the following terms have been used to indicate the assessed likelihood of an outcome or a result: Virtually certain 99-100\% probability, Very likely 90-100\%, Likely $66-100 \%$, About as likely as not 33-66\%, Unlikely 0-33\%, Very unlikely 0-10\%, Exceptionally unlikely 0-1\%. Additional terms (Extremely likely: 95-100\%, More likely than not $>50-100 \%$, and Extremely unlikely 0-5\%) may also be used when appropriate. Assessed likelihood is typeset in italics, e.g., very likely (see Section 1.4 and Box TS.1 for more details).

2 In this Report, the following summary terms are used to describe the available evidence: limited, medium, or robust; and for the degree of agreement: low, medium, or high. A level of confidence is expressed using five qualifiers: very low, low, medium, high, and very high, and typeset in italics, e.g., medium confidence. For a given evidence and agreement statement, different confidence levels can be assigned, but increasing levels of evidence and degrees of agreement are correlated with increasing confidence (see Section 1.4 and Box TS.1 for more details). 
The observed recent warming hiatus, defined as the reduction in GMST trend during 1998-2012 as compared to the trend during 1951-2012, is attributable in roughly equal measure to a cooling contribution from internal variability and a reduced trend in external forcing (expert judgement, medium confidence). The forcing trend reduction is primarily due to a negative forcing trend from both volcanic eruptions and the downward phase of the solar cycle. However, there is low confidence in quantifying the role of forcing trend in causing the hiatus because of uncertainty in the magnitude of the volcanic forcing trends and low confidence in the aerosol forcing trend. Many factors, in addition to GHGs, including changes in tropospheric and stratospheric aerosols, stratospheric water vapour, and solar output, as well as internal modes of variability, contribute to the year-to-year and decade- to-decade variability of GMST. \{Box 9.2, 10.3.1, Figure 10.6\}

\section{Ocean Temperatures and Sea Level Rise}

It is very likely that anthropogenic forcings have made a substantial contribution to upper ocean warming (above $700 \mathrm{~m}$ ) observed since the 1970s. This anthropogenic ocean warming has contributed to global sea level rise over this period through thermal expansion. New understanding since AR4 of measurement errors and their correction in the temperature data sets have increased the agreement in estimates of ocean warming. Observations of ocean warming are consistent with climate model simulations that include anthropogenic and volcanic forcings but are inconsistent with simulations that exclude anthropogenic forcings. Simulations that include both anthropogenic and natural forcings have decadal variability that is consistent with observations. These results are a major advance on AR4. \{3.2.3, 10.4.1, Table 10.1\}

It is very likely that there is a substantial contribution from anthropogenic forcings to the global mean sea level rise since the 1970s. It is likely that sea level rise has an anthropogenic contribution from Greenland melt since 1990 and from glacier mass loss since 1960s. Observations since 1971 indicate with high confidence that thermal expansion and glaciers (excluding the glaciers in Antarctica) explain $75 \%$ of the observed rise. $\{10.4 .1,10.4 .3,10.5 .2$, Table 10.1, 13.3.6\}

\section{Ocean Acidification and Oxygen Change}

It is very likely that oceanic uptake of anthropogenic carbon dioxide has resulted in acidification of surface waters which is observed to be between -0.0014 and $-0.0024 \mathrm{pH}$ units per year. There is medium confidence that the observed global pattern of decrease in oxygen dissolved in the oceans from the 1960s to the 1990s can be attributed in part to human influences. \{3.8.2, Box 3.2, 10.4.4, Table 10.1\}

\section{The Water Cycle}

New evidence is emerging for an anthropogenic influence on global land precipitation changes, on precipitation increases in high northern latitudes, and on increases in atmospheric humidity. There is medium confidence that there is an anthropogenic contribution to observed increases in atmospheric specific humidity since 1973 and to global scale changes in precipitation patterns over land since 1950, including increases in NH mid to high latitudes. Remaining observational and modelling uncertainties, and the large internal variability in precipitation, preclude a more confident assessment at this stage. $\{2.5 .1,2.5 .4,10.3 .2$, Table 10.1$\}$

It is very likely that anthropogenic forcings have made a discernible contribution to surface and subsurface oceanic salinity changes since the 1960s. More than 40 studies of regional and global surface and subsurface salinity show patterns consistent with understanding of anthropogenic changes in the water cycle and ocean circulation. The expected pattern of anthropogenic amplification of climatological salinity patterns derived from climate models is detected in the observations although there remains incomplete understanding of the observed internal variability of the surface and sub-surface salinity fields. $\{3.3 .2,10.4 .2$, Table 10.1$\}$

It is likely that human influence has affected the global water cycle since 1960. This assessment is based on the combined evidence from the atmosphere and oceans of observed systematic changes that are attributed to human influence in terrestrial precipitation, atmospheric humidity and oceanic surface salinity through its connection to precipitation and evaporation. This is a major advance since AR4. $\{3.3 .2,10.3 .2,10.4 .2$, Table 10.1\}

\section{Cryosphere}

Anthropogenic forcings are very likely to have contributed to Arctic sea ice loss since 1979. There is a robust set of results from simulations that show the observed decline in sea ice extent is simulated only when models include anthropogenic forcings. There is low confidence in the scientific understanding of the observed increase in Antarctic sea ice extent since 1979 owing to the incomplete and competing scientific explanations for the causes of change and low confidence in estimates of internal variability. $\{10.5 .1$, Table 10.1\}

Ice sheets and glaciers are melting, and anthropogenic influences are likely to have contributed to the surface melting of Greenland since 1993 and to the retreat of glaciers since the 1960s. Since 2007, internal variability is likely to have further enhanced the melt over Greenland. For glaciers there is a high level of scientific understanding from robust estimates of observed mass loss, internal variability and glacier response to climatic drivers. Owing to a low level of scientific understanding there is low confidence in attributing the causes of the observed loss of mass from the Antarctic ice sheet since 1993. $\{4.3 .3,10.5 .2$, Table 10.1\}

It is likely that there has been an anthropogenic component to observed reductions in NH snow cover since 1970. There is high agreement across observations studies and attribution studies find a human influence at both continental and regional scales. $\{10.5 .3$, Table $10.1\}$ 


\section{Climate Extremes}

There has been a strengthening of the evidence for human influence on temperature extremes since the AR4 and IPCC Special Report on Managing the Risks of Extreme Events and Disasters to Advance Climate Change Adaptation (SREX) reports. It is very likely that anthropogenic forcing has contributed to the observed changes in the frequency and intensity of daily temperature extremes on the global scale since the mid-20th century. Attribution of changes in temperature extremes to anthropogenic influence is robustly seen in independent analyses using different methods and different data sets. It is likely that human influence has substantially increased the probability of occurrence of heatwaves in some locations. \{10.6.1, 10.6.2, Table 10.1\}

In land regions where observational coverage is sufficient for assessment, there is medium confidence that anthropogenic forcing has contributed to a global-scale intensification of heavy precipitation over the second half of the 20th century. There is low confidence in attributing changes in drought over global land areas since the mid-20th century to human influence owing to observational uncertainties and difficulties in distinguishing decadal-scale variability in drought from long-term trends. \{10.6.1, Table $10.1\}$

There is low confidence in attribution of changes in tropical cyclone activity to human influence owing to insufficient observational evidence, lack of physical understanding of the links between anthropogenic drivers of climate and tropical cyclone activity and the low level of agreement between studies as to the relative importance of internal variability, and anthropogenic and natural forcings. This assessment is consistent with that of SREX. \{10.6.1, Table 10.1\}

\section{Atmospheric Circulation}

It is likely that human influence has altered sea level pressure patterns globally. Detectable anthropogenic influence on changes in sea level pressure patterns is found in several studies. Changes in atmospheric circulation are important for local climate change since they could lead to greater or smaller changes in climate in a particular region than elsewhere. There is medium confidence that stratospheric ozone depletion has contributed to the observed poleward shift of the southern Hadley Cell border during austral summer. There are large uncertainties in the magnitude of this poleward shift. It is likely that stratospheric ozone depletion has contributed to the positive trend in the Southern Annular Mode seen in austral summer since the mid20th century which corresponds to sea level pressure reductions over the high latitudes and an increase in the subtropics. There is medium confidence that GHGs have also played a role in these trends of the southern Hadley Cell border and the Southern Annular Mode in Austral summer. $\{10.3 .3$, Table 10.1\}

\section{A Millennia to Multi-Century Perspective}

Taking a longer term perspective shows the substantial role played by anthropogenic and natural forcings in driving climate variability on hemispheric scales prior to the twentieth century. It is very unlikely that $\mathrm{NH}$ temperature variations from 1400 to 1850 can be explained by internal variability alone. There is medium confidence that external forcing contributed to $\mathrm{NH}$ temperature variability from 850 to 1400 and that external forcing contributed to European temperature variations over the last five centuries. $\{10.7 .2,10.7 .5$, Table 10.1\}

\section{Climate System Properties}

The extended record of observed climate change has allowed a better characterization of the basic properties of the climate system that have implications for future warming. New evidence from 21st century observations and stronger evidence from a wider range of studies have strengthened the constraint on the transient climate response (TCR) which is estimated with high confidence to be likely between $1{ }^{\circ} \mathrm{C}$ and $2.5^{\circ} \mathrm{C}$ and extremely unlikely to be greater than $3^{\circ} \mathrm{C}$. The Transient Climate Response to Cumulative $\mathrm{CO}_{2}$ Emissions (TCRE) is estimated with high confidence to be likely between $0.8^{\circ} \mathrm{C}$ and $2.5^{\circ} \mathrm{C}$ per $1000 \mathrm{PgC}$ for cumulative $\mathrm{CO}_{2}$ emissions less than about $2000 \mathrm{PgC}$ until the time at which temperatures peak. Estimates of the Equilibrium Climate Sensitivity (ECS) based on multiple and partly independent lines of evidence from observed climate change indicate that there is high confidence that ECS is extremely unlikely to be less than $1^{\circ} \mathrm{C}$ and medium confidence that the ECS is likely to be between $1.5^{\circ} \mathrm{C}$ and $4.5^{\circ} \mathrm{C}$ and very unlikely greater than $6^{\circ} \mathrm{C}$. These assessments are consistent with the overall assessment in Chapter 12, where the inclusion of additional lines of evidence increases confidence in the assessed likely range for ECS. \{10.8.1, 10.8.2, 10.8.4, Box 12.2\}

\section{Combination of Evidence}

Human influence has been detected in the major assessed components of the climate system. Taken together, the combined evidence increases the level of confidence in the attribution of observed climate change, and reduces the uncertainties associated with assessment based on a single climate variable. From this combined evidence it is virtually certain that human influence has warmed the global climate system. Anthropogenic influence has been identified in changes in temperature near the surface of the Earth, in the atmosphere and in the oceans, as well as changes in the cryosphere, the water cycle and some extremes. There is strong evidence that excludes solar forcing, volcanoes and internal variability as the strongest drivers of warming since 1950. \{10.9.2, Table 10.1\} 


\subsection{Introduction}

This chapter assesses the causes of observed changes assessed in Chapters 2 to 5 and uses understanding of physical processes, climate models and statistical approaches. The chapter adopts the terminology for detection and attribution proposed by the IPCC good practice guidance paper on detection and attribution (Hegerl et al., 2010) and for uncertainty Mastrandrea et al. (2011). Detection and attribution of impacts of climate changes are assessed by Working Group II, where Chapter 18 assesses the extent to which atmospheric and oceanic changes influence ecosystems, infrastructure, human health and activities in economic sectors.

Evidence of a human influence on climate has grown stronger over the period of the four previous assessment reports of the IPCC. There was little observational evidence for a detectable human influence on climate at the time of the First IPCC Assessment Report. By the time of the second report there was sufficient additional evidence for it to conclude that 'the balance of evidence suggests a discernible human influence on global climate'. The Third Assessment Report found that a distinct greenhouse gas (GHG) signal was robustly detected in the observed temperature record and that 'most of the observed warming over the last fifty years is likely to have been due to the increase in greenhouse gas concentrations.'

With the additional evidence available by the time of the Fourth Assessment Report, the conclusions were further strengthened. This evidence included a wider range of observational data, a greater variety of more sophisticated climate models including improved representations of forcings and processes and a wider variety of analysis techniques. This enabled the AR4 report to conclude that 'most of the observed increase in global average temperatures since the mid-20th century is very likely due to the observed increase in anthropogenic greenhouse gas concentrations'. The AR4 also concluded that 'discernible human influences now extend to other aspects of climate, including ocean warming, continental-average temperatures, temperature extremes and wind patterns.'

A number of uncertainties remained at the time of AR4. For example, the observed variability of ocean temperatures appeared inconsistent with climate models, thereby reducing the confidence with which observed ocean warming could be attributed to human influence. Also, although observed changes in global rainfall patterns and increases in heavy precipitation were assessed to be qualitatively consistent with expectations of the response to anthropogenic forcings, detection and attribution studies had not been carried out. Since the AR4, improvements have been made to observational data sets, taking more complete account of systematic biases and inhomogeneities in observational systems, further developing uncertainty estimates, and correcting detected data problems (Chapters 2 and 3). A new set of simulations from a greater number of AOGCMs have been performed as part of the Coupled Model Intercomparison Project Phase 5 (CMIP5). These new simulations have several advantages over the CMIP3 simulations assessed in the AR4 (Hegerl et al., 2007b). They incorporate some moderate increases in resolution, improved parameterizations, and better representation of aerosols (Chapter 9). Importantly for attri- bution, in which it is necessary to partition the response of the climate system to different forcings, most CMIP5 models include simulations of the response to natural forcings only, and the response to increases in well mixed GHGs only (Taylor et al., 2012).

The advances enabled by this greater wealth of observational and model data are assessed in this chapter. In this assessment, there is increased focus on the extent to which the climate system as a whole is responding in a coherent way across a suite of climate variables such as surface mean temperature, temperature extremes, ocean heat content, ocean salinity and precipitation change. There is also a global to regional perspective, assessing the extent to which not just global mean changes but also spatial patterns of change across the globe can be attributed to anthropogenic and natural forcings.

\subsection{Evaluation of Detection and Attribution Methodologies}

Detection and attribution methods have been discussed in previous assessment reports (Hegerl et al., 2007b) and the IPCC Good Practice Guidance Paper (Hegerl et al., 2010), to which we refer. This section reiterates key points and discusses new developments and challenges.

\subsubsection{The Context of Detection and Attribution}

In IPCC Assessments, detection and attribution involve quantifying the evidence for a causal link between external drivers of climate change and observed changes in climatic variables. It provides the central, although not the only (see Section 1.2.3) line of evidence that has supported statements such as 'the balance of evidence suggests a discernible human influence on global climate' or 'most of the observed increase in global average temperatures since the mid-20th century is very likely due to the observed increase in anthropogenic greenhouse gas concentrations.'

The definition of detection and attribution used here follows the terminology in the IPCC guidance paper (Hegerl et al., 2010). 'Detection of change is defined as the process of demonstrating that climate or a system affected by climate has changed in some defined statistical sense without providing a reason for that change. An identified change is detected in observations if its likelihood of occurrence by chance due to internal variability alone is determined to be small' (Hegerl et al., 2010). Attribution is defined as 'the process of evaluating the relative contributions of multiple causal factors to a change or event with an assignment of statistical confidence'. As this wording implies, attribution is more complex than detection, combining statistical analysis with physical understanding (Allen et al., 2006; Hegerl and Zwiers, 2011). In general, a component of an observed change is attributed to a specific causal factor if the observations can be shown to be consistent with results from a process-based model that includes the causal factor in question, and inconsistent with an alternate, otherwise identical, model that excludes this factor. The evaluation of this consistency in both of these cases takes into account internal chaotic variability and known uncertainties in the observations and responses to external causal factors. 
Attribution does not require, and nor does it imply, that every aspect of the response to the causal factor in question is simulated correctly. Suppose, for example, the global cooling following a large volcano matches the cooling simulated by a model, but the model underestimates the magnitude of this cooling: the observed global cooling can still be attributed to that volcano, although the error in magnitude would suggest that details of the model response may be unreliable. Physical understanding is required to assess what constitutes a plausible discrepancy above that expected from internal variability. Even with complete consistency between models and data, attribution statements can never be made with $100 \%$ certainty because of the presence of internal variability.

This definition of attribution can be extended to include antecedent conditions and internal variability among the multiple causal factors contributing to an observed change or event. Understanding the relative importance of internal versus external factors is important in the analysis of individual weather events (Section 10.6.2), but the primary focus of this chapter will be on attribution to factors external to the climate system, like rising GHG levels, solar variability and volcanic activity.

There are four core elements to any detection and attribution study:

1. Observations of one or more climate variables, such as surface temperature, that are understood, on physical grounds, to be relevant to the process in question

2. An estimate of how external drivers of climate change have evolved before and during the period under investigation, including both the driver whose influence is being investigated (such as rising GHG levels) and potential confounding influences (such as solar activity)

3. A quantitative physically based understanding, normally encapsulated in a model, of how these external drivers are thought to have affected these observed climate variables

4. An estimate, often but not always derived from a physically based model, of the characteristics of variability expected in these observed climate variables due to random, quasi-periodic and chaotic fluctuations generated in the climate system that are not due to externally driven climate change

A climate model driven with external forcing alone is not expected to replicate the observed evolution of internal variability, because of the chaotic nature of the climate system, but it should be able to capture the statistics of this variability (often referred to as 'noise'). The reliability of forecasts of short-term variability is also a useful test of the representation of relevant processes in the models used for attribution, but forecast skill is not necessary for attribution: attribution focuses on changes in the underlying moments of the 'weather attractor', meaning the expected weather and its variability, while prediction focuses on the actual trajectory of the weather around this attractor.

In proposing that 'the process of attribution requires the detection of a change in the observed variable or closely associated variables' (Hegerl et al., 2010), the new guidance recognized that it may be possible, in some instances, to attribute a change in a particular variable to some external factor before that change could actually be detected in the variable itself, provided there is a strong body of knowledge that links a change in that variable to some other variable in which a change can be detected and attributed. For example, it is impossible in principle to detect a trend in the frequency of 1-in-100-year events in a 100-year record, yet if the probability of occurrence of these events is physically related to large-scale temperature changes, and we detect and attribute a large-scale warming, then the new guidance allows attribution of a change in probability of occurrence before such a change can be detected in observations of these events alone. This was introduced to draw on the strength of attribution statements from, for example, time-averaged temperatures, to attribute changes in closely related variables.

Attribution of observed changes is not possible without some kind of model of the relationship between external climate drivers and observable variables. We cannot observe a world in which either anthropogenic or natural forcing is absent, so some kind of model is needed to set up and evaluate quantitative hypotheses: to provide estimates of how we would expect such a world to behave and to respond to anthropogenic and natural forcings (Hegerl and Zwiers, 2011). Models may be very simple, just a set of statistical assumptions, or very complex, complete global climate models: it is not necessary, or possible, for them to be correct in all respects, but they must provide a physically consistent representation of processes and scales relevant to the attribution problem in question.

One of the simplest approaches to detection and attribution is to compare observations with model simulations driven with natural forcings alone, and with simulations driven with all relevant natural and anthropogenic forcings. If observed changes are consistent with simulations that include human influence, and inconsistent with those that do not, this would be sufficient for attribution providing there were no other confounding influences and it is assumed that models are simulating the responses to all external forcings correctly. This is a strong assumption, and most attribution studies avoid relying on it. Instead, they typically assume that models simulate the shape of the response to external forcings (meaning the large-scale pattern in space and/or time) correctly, but do not assume that models simulate the magnitude of the response correctly. This is justified by our fundamental understanding of the origins of errors in climate modelling. Although there is uncertainty in the size of key forcings and the climate response, the overall shape of the response is better known: it is set in time by the timing of emissions and set in space (in the case of surface temperatures) by the geography of the continents and differential responses of land and ocean (see Section 10.3.1.1.2).

So-called 'fingerprint' detection and attribution studies characterize their results in terms of a best estimate and uncertainty range for 'scaling factors' by which the model-simulated responses to individual forcings can be scaled up or scaled down while still remaining consistent with the observations, accounting for similarities between the patterns of response to different forcings and uncertainty due to internal climate variability. If a scaling factor is significantly larger than zero (at some significance level), then the response to that forcing, as simulated by 
that model and given that estimate of internal variability and other potentially confounding responses, is detectable in these observations, whereas if the scaling factor is consistent with unity, then that model-simulated response is consistent with observed changes. Studies do not require scaling factors to be consistent with unity for attribution, but any discrepancy from unity should be understandable in terms of known uncertainties in forcing or response: a scaling factor of 10, for example, might suggest the presence of a confounding factor, calling into question any attribution claim. Scaling factors are estimated by fitting model-simulated responses to observations, so results are unaffected, at least to first order, if the model has a transient climate response, or aerosol forcing, that is too low or high. Conversely, if the spatial or temporal pattern of forcing or response is wrong, results can be affected: see Box 10.1 and further discussion in Section 10.3.1.1 and Hegerl and Zwiers (2011) and Hegerl et al. (2011b). Sensitivity of results to the pattern of forcing or response can be assessed by comparing results across multiple models or by representing pattern uncertainty explicitly (Huntingford et al., 2006), but errors that are common to all models (through limited vertical resolution, for example) will not be addressed in this way and are accounted for in this assessment by downgrading overall assessed likelihoods to be generally more conservative than the quantitative likelihoods provided by individual studies.

10 Attribution studies must compromise between estimating responses to different forcings separately, which allows for the possibility of different errors affecting different responses (errors in aerosol forcing that do not affect the response to GHGs, for example), and estimating responses to combined forcings, which typically gives smaller uncertainties because it avoids the issue of 'degeneracy': if two responses have very similar shapes in space and time, then it may be impossible to estimate the magnitude of both from a single set of observations because amplification of one may be almost exactly compensated for by amplification or diminution of the other (Allen et al., 2006). Many studies find it is possible to estimate the magnitude of the responses to GHG and other anthropogenic forcings separately, particularly when spatial information is included. This is important, because it means the estimated response to GHG increase is not dependent on the uncertain magnitude of forcing and response due to aerosols (Hegerl et al., 2011b).

The simplest way of fitting model-simulated responses to observations is to assume that the responses to different forcings add linearly, so the response to any one forcing can be scaled up or down without affecting any of the others and that internal climate variability is independent of the response to external forcing. Under these conditions, attribution can be expressed as a variant of linear regression (see Box 10.1). The additivity assumption has been tested and found to hold for large-scale temperature changes (Meehl et al., 2003; Gillett et al., 2004) but it might not hold for other variables like precipitation (Hegerl et al., 2007b; Hegerl and Zwiers, 2011; Shiogama et al., 2012), nor for regional temperature changes (Terray, 2012). In principle, additivity is not required for detection and attribution, but to date non-additive approaches have not been widely adopted.

The estimated properties of internal climate variability play a central role in this assessment. These are either estimated empirically from the observations (Section 10.2.2) or from paleoclimate reconstructions
(Section 10.7.1) (Esper et al., 2012) or derived from control simulations of coupled models (Section 10.2.3). The majority of studies use modelled variability and routinely check that the residual variability from observations is consistent with modelled internal variability used over time scales shorter than the length of the instrumental record (Allen and Tett, 1999). Assessing the accuracy of model-simulated variability on longer time scales using paleoclimate reconstructions is complicated by the fact that some reconstructions may not capture the full spectrum of variability because of limitations of proxies and reconstruction methods, and by the unknown role of external forcing in the pre-instrumental record. In general, however, paleoclimate reconstructions provide no clear evidence either way whether models are over- or underestimating internal variability on time scales relevant for attribution (Esper et al., 2012; Schurer et al., 2013).

\subsubsection{Time Series Methods, Causality and Separating Signal from Noise}

Some studies attempt to distinguish between externally driven climate change and changes due to internal variability minimizing the use of climate models, for example, by separating signal and noise by time scale (Schneider and Held, 2001), spatial pattern (Thompson et al., 2009) or both. Other studies use model control simulations to identify patterns of maximum predictability and contrast these with the forced component in climate model simulations (DelSole et al., 2011): see Section 10.3.1. Conclusions of most studies are consistent with those based on fingerprint detection and attribution, while using a different set of assumptions (see review in Hegerl and Zwiers, 2011).

A number of studies have applied methods developed in the econometrics literature (Engle and Granger, 1987) to assess the evidence for a causal link between external drivers of climate and observed climate change, using the observations themselves to estimate the expected properties of internal climate variability (e.g., Kaufmann and Stern, 1997). The advantage of these approaches is that they do not depend on the accuracy of any complex global climate model, but they nevertheless have to assume some kind of model, or restricted class of models, of the properties of the variables under investigation. Attribution is impossible without a model: although this model may be implicit in the statistical framework used, it is important to assess its physical consistency (Kaufmann et al., 2013). Many of these time series methods can be cast in the overall framework of co-integration and error correction (Kaufmann et al., 2011), which is an approach to analysing relationships between stationary and non-stationary time series. If there is a consistent causal relationship between two or more possibly non-stationary time series, then it should be possible to find a linear combination such that the residual is stationary (contains no stochastic trend) over time (Kaufmann and Stern, 2002; Kaufmann et al., 2006; Mills, 2009). Co-integration methods are thus similar in overall principle to regression-based approaches (e.g., Douglass et al., 2004; Stone and Allen, 2005; Lean, 2006) to the extent that regression studies take into account the expected time series properties of the data-the example described in Box 10.1 might be characterized as looking for a linear combination of anthropogenic and natural forcings such that the observed residuals were consistent with internal climate variability as simulated by the CMIP5 models. Co-integration and error correction methods, however, generally make more explicit use of time 


\section{Box 10.1 | How Attribution Studies Work}

This box presents an idealized demonstration of the concepts underlying most current approaches to detection and attribution of climate change and how these relate to conventional linear regression. The coloured dots in Box 10.1a, Figure 1 show observed annual GMST from 1861 to 2012, with warmer years coloured red and colder years coloured blue. Observations alone indicate, unequivocally, that the Earth has warmed, but to quantify how different external factors have contributed to this warming, studies must compare such observations with the expected responses to these external factors. The orange line shows an estimate of the GMST response to anthropogenic (GHG and aerosol) forcing obtained from the mean of the CMIP3 and CMIP5 ensembles, while the blue line shows the CMIP3/CMIP5 ensemble mean response to natural (solar and volcanic) forcing.

In statistical terms, attribution involves finding the combination of these anthropogenic and natural responses that best fits these observations: this is shown by the black line in panel (a). To show how this fit is obtained in non-technical terms, the data are plotted against model-simulated anthropogenic warming, instead of time, in panel (b). There is a strong correlation between observed temperatures and model-simulated anthropogenic warming, but because of the presence of natural factors and internal climate variability, correlation alone is not enough for attribution.

To quantify how much of the observed warming is attributable to human influence, panel (c) shows observed temperatures plotted against the model-simulated response to anthropogenic forcings in one direction and natural forcings in the other. Observed temperatures increase with both natural and anthropogenic model-simulated warming: the warmest years are in the far corner of the box. A flat surface through these points (here obtained by an ordinary least-squares fit), indicated by the coloured mesh, slopes up away from the viewer.

The orientation of this surface indicates how model-simulated responses to natural and anthropogenic forcing need to be scaled to reproduce the observations. The best-fit gradient in the direction of anthropogenic warming (visible on the rear left face of the box) is 0.9 , indicating the CMIP3/CMIP5 ensemble average overestimates the magnitude of the observed response to anthropogenic forcing by about $10 \%$. The best-fit gradient in the direction of natural changes (visible on the rear right face) is 0.7 , indicating that the observed response to natural forcing is $70 \%$ of the average model-simulated response. The black line shows the points on this flat surface that are directly above or below the observations: each 'pin' corresponds to a different year. When re-plotted against time, indicated by the years on the rear left face of the box, this black line gives the black line previously seen in panel (a). The length of the pins indicates 'residual' temperature fluctuations due to internal variability.

The timing of these residual temperature fluctuations is unpredictable, representing an inescapable source of uncertainty. We can quantify this uncertainty by asking how the gradients of the best-fit surface might vary if El Niño events, for example, had occurred in different years in the observed temperature record. To do this, we repeat the analysis in panel (c), replacing observed temperatures with samples of simulated internal climate variability from control runs of coupled climate models. Grey diamonds in panel (d) show the results: these gradients cluster around zero, because control runs have no anthropogenic or natural forcing, but there is still some scatter. Assuming that internal variability in global temperature simply adds to the response to external forcing, this scatter provides an estimate of uncertainty in the gradients, or scaling factors, required to reproduce the observations, shown by the red cross and ellipse.

The red cross and ellipse are clearly separated from the origin, which means that the slope of the best-fit surface through the observations cannot be accounted for by internal variability: some climate change is detected in these observations. Moreover, it is also separated from both the vertical and horizontal axes, which means that the responses to both anthropogenic and natural factors are individually detectable.

The magnitude of observed temperature change is consistent with the CMIP3/CMIP5 ensemble average response to anthropogenic forcing (uncertainty in this scaling factor spans unity) but is significantly lower than the model-average response to natural forcing (this 5 to $95 \%$ confidence interval excludes unity). There are, however, reasons why these models may be underestimating the response to volcanic forcing (e.g., Driscoll et al, 2012), so this discrepancy does not preclude detection and attribution of both anthropogenic and natural influence, as simulated by the CMIP3/CMIP5 ensemble average, in the observed GMST record.

The top axis in panel (d) indicates the attributable anthropogenic warming over 1951-2010, estimated from the anthropogenic warming in the CMIP3/CMIP5 ensemble average, or the gradient of the orange line in panel (a) over this period. Because the model-simulated responses have been scaled to fit the observations, the attributable anthropogenic warming in this example is $0.6^{\circ} \mathrm{C}$ to $0.9^{\circ} \mathrm{C}$ and does not depend on the magnitude of the raw model-simulated changes. Hence an attribution statement based on such an analysis, 


\section{Box 10.1 (continued)}

such as 'most of the warming over the past 50 years is attributable to anthropogenic drivers', depends only on the shape, or time history, not the size, of the model-simulated warming, and hence does not depend on the models' sensitivity to rising GHG levels.

Formal attribution studies like this example provide objective estimates of how much recent warming is attributable to human influence. Attribution is not, however, a purely statistical exercise. It also requires an assessment that there are no confounding factors that could have caused a large part of the 'attributed' change. Statistical tests can be used to check that observed residual temperature fluctuations (the lengths and clustering of the pins in panel (c)) are consistent with internal variability expected from coupled models, but ultimately these tests must complement physical arguments that the combination of responses to anthropogenic and natural forcing is the only available consistent explanation of recent observed temperature change.

This demonstration assumes, for visualization purposes, that there are only two candidate contributors to the observed warming, anthropogenic and natural, and that only GMST is available. More complex attribution problems can be undertaken using the same principles, such as aiming to separate the response to GHGs from other anthropogenic factors by also including spatial information. These require, in effect, an extension of panel (c), with additional dimensions corresponding to additional causal factors, and additional points corresponding to temperatures in different regions.
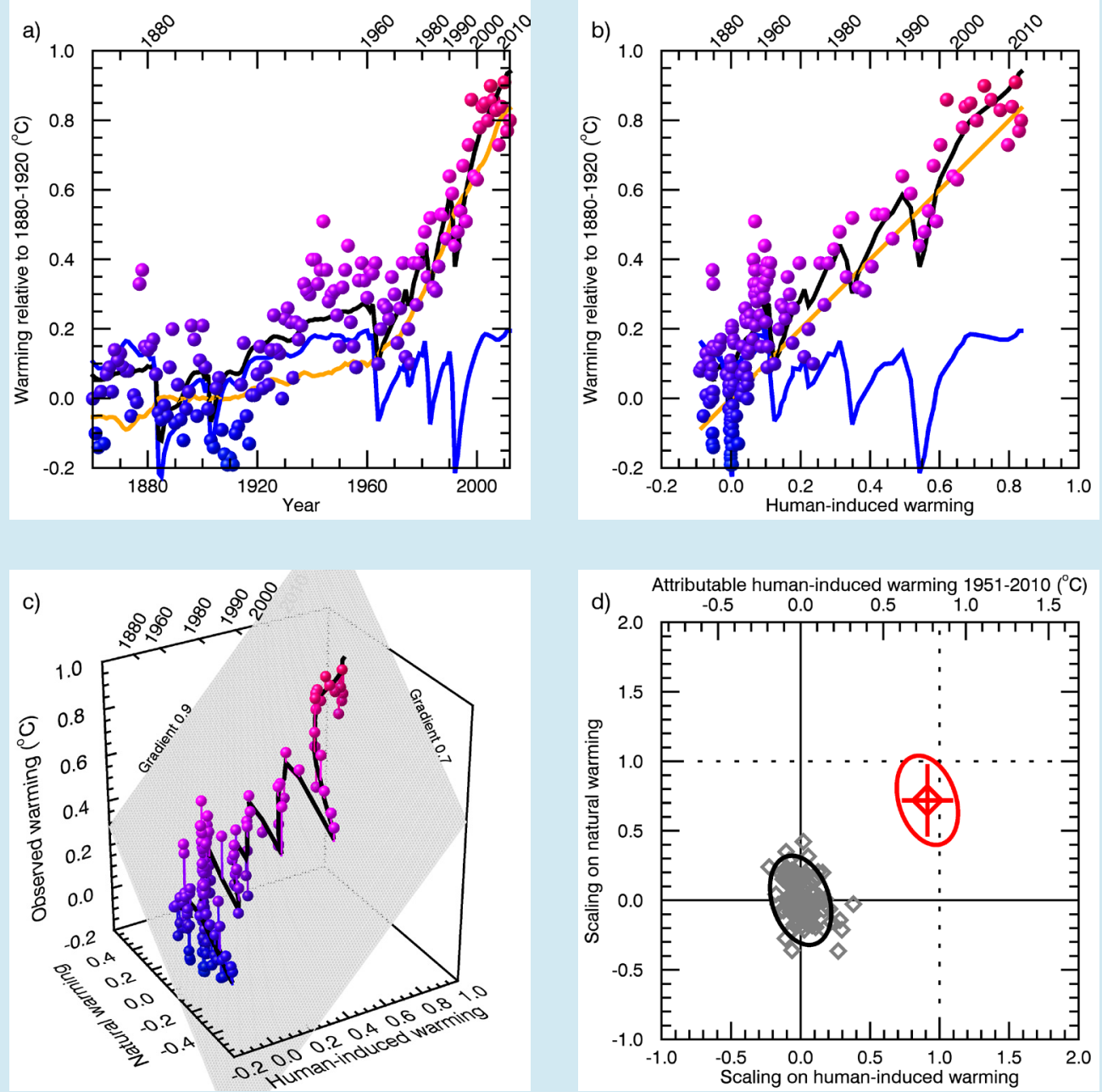

Box 10.1, Figure 1 | Example of a simplified detection and attribution study. (a) Observed global annual mean temperatures relative to 1880-1920 (coloured dots) compared with CMIP3/CMIP5 ensemble-mean response to anthropogenic forcing (orange), natural forcing (blue) and best-fit linear combination (black). (b) As (a) but all data plotted against model-simulated anthropogenic warming in place of time. Selected years (increasing nonlinearly) shown on top axis. (c) Observed temperatures versus model-simulated anthropogenic and natural temperature changes, with best-fit plane shown by coloured mesh. (d) Gradient of best-fit plane in (c), or scaling on model-simulated responses required to fit observations (red diamond) with uncertainty estimate (red ellipse and cross) based on CMIP5 control integrations (grey diamonds). Implied attributable anthropogenic warming over the period 1951-2010 is indicated by the top axis. Anthropogenic and natural responses are noise-reduced with 5-point running means, with no smoothing over years with major volcanoes. 
series properties (notice how date information is effectively discarded in panel (b) of Box 10.1, Figure 1) and require fewer assumptions about the stationarity of the input series.

All of these approaches are subject to the issue of confounding factors identified by Hegerl and Zwiers (2011). For example, Beenstock et al. (2012) fail to find a consistent co-integrating relationship between atmospheric carbon dioxide $\left(\mathrm{CO}_{2}\right)$ concentrations and GMST using polynomial cointegration tests, but the fact that $\mathrm{CO}_{2}$ concentrations are derived from different sources in different periods (ice cores prior to the mid-20th-century, atmospheric observations thereafter) makes it difficult to assess the physical significance of their result, particularly in the light of evidence for co-integration between temperature and radiative forcing (RF) reported by Kaufmann et al. (2011) using tests of linear cointegration, and also the results of Gay-Garcia et al. (2009), who find evidence for external forcing of climate using time series properties.

The assumptions of the statistical model employed can also influence results. For example, Schlesinger and Ramankutty (1994) and Zhou and Tung (2013a) show that GMST are consistent with a linear anthropogenic trend, enhanced variability due to an approximately 70-year Atlantic Meridional Oscillation (AMO) and shorter-term variability. If, however, there are physical grounds to expect a nonlinear anthropogenic trend (see Box 10.1 Figure 1a), the assumption of a linear trend can itself enhance the variance assigned to a low-frequency oscillation. The fact that the AMO index is estimated from detrended historical temperature observations further increases the risk that its variance may be overestimated, because regressors and regressands are not independent. Folland et al. (2013), using a physically based estimate of the anthropogenic trend, find a smaller role for the AMO in recent warming.

Time series methods ultimately depend on the structural adequacy of the statistical model employed. Many such studies, for example, use models that assume a single exponential decay time for the response to both external forcing and stochastic fluctuations. This can lead to an overemphasis on short-term fluctuations, and is not consistent with the response of more complex models (Knutti et al., 2008). Smirnov and Mokhov (2009) propose an alternative characterization that allows them to distinguish a 'long-term causality' that focuses on low-frequency changes. Trends that appear significant when tested against an $A R(1)$ model may not be significant when tested against a process that supports this 'long-range dependence' (Franzke, 2010). Although the evidence for long-range dependence in global temperature data remains a topic of debate (Mann, 2011; Rea et al., 2011) , it is generally desirable to explore sensitivity of results to the specification of the statistical model, and also to other methods of estimating the properties of internal variability, such as more complex climate models, discussed next. For example, Imbers et al. (2013) demonstrate that the detection of the influence of increasing GHGs in the global temperature record is robust to the assumption of a Fractional Differencing (FD) model of internal variability, which supports long-range dependence.

\subsubsection{Methods Based on General Circulation Models and Optimal Fingerprinting}

Fingerprinting methods use climate model simulations to provide more complete information about the expected response to different external drivers, including spatial information, and the properties of internal climate variability. This can help to separate patterns of forced change both from each other and from internal variability. The price, however, is that results depend to some degree on the accuracy of the shape of model-simulated responses to external factors (e.g., North and Stevens, 1998), which is assessed by comparing results obtained with expected responses estimated from different climate models. When the signal-to-noise $(S / N)$ ratio is low, as can be the case for some regional indicators and some variables other than temperature, the accuracy of the specification of variability becomes a central factor in the reliability of any detection and attribution study. Many studies of such variables inflate the variability estimate from models to determine if results are sensitive to, for example, doubling of variance in the control (e.g., Zhang et al., 2007), although Imbers et al. (2013) note that errors in the spectral properties of simulated variability may also be important.

A full description of optimal fingerprinting is provided in Appendix 9.A of Hegerl et al. (2007b) and further discussion is to be found in Hasselmann (1997), Allen and Tett (1999), Allen et al. (2006), and Hegerl and Zwiers (2011). Box 10.1 provides a simple example of 'fingerprinting' based on GMST alone. In a typical fingerprint analysis, model-simulated spatio-temporal patterns of response to different combinations of external forcings, including segments of control integrations with no forcing, are 'observed' in a similar manner to the historical record (masking out times and regions where observations are absent). The magnitudes of the model-simulated responses are then estimated in the observations using a variant of linear regression, possibly allowing for signals being contaminated by internal variability (Allen and Stott, 2003) and structural model uncertainty (Huntingford et al., 2006).

In 'optimal' fingerprinting, model-simulated responses and observations are normalized by internal variability to improve the $S / N$ ratio. This requires an estimate of the inverse noise covariance estimated from the sample covariance matrix of a set of unforced (control) simulations (Hasselmann, 1997), or from variations within an initial-condition ensemble. Because these control runs are generally too short to estimate the full covariance matrix, a truncated version is used, retaining only a small number, typically of order 10 to 20 , of high-variance principal components. Sensitivity analyses are essential to ensure results are robust to this, relatively arbitrary, choice of truncation (Allen and Tett, 1999; Ribes and Terray, 2013; Jones et al., 2013 ). Ribes et al. (2009) use a regularized estimate of the covariance matrix, meaning a linear combination of the sample covariance matrix and a unit matrix that has been shown (Ledoit and Wolf, 2004) to provide a more accurate estimate of the true covariance, thereby avoiding dependence on truncation. Optimization of $\mathrm{S} / \mathrm{N}$ ratio is not, however, essential for many attribution results (see, e.g., Box 10.1) and uncertainty analysis in conventional optimal fingerprinting does not require the covariance matrix to be inverted, so although regularization may help in some cases, it is not essential. Ribes et al. (2010) also propose a hybrid of the model-based optimal fingerprinting and time series approaches, referred to as 'temporal optimal detection', under which each signal is assumed to consist of a single spatial pattern modulated by a smoothly varying time series estimated from a climate model (see also Santer et al., 1994). 
The final statistical step in an attribution study is to check that the residual variability, after the responses to external drivers have been estimated and removed, is consistent with the expected properties of internal climate variability, to ensure that the variability used for uncertainty analysis is realistic, and that there is no evidence that a potentially confounding factor has been omitted. Many studies use a standard $F$-test of residual consistency for this purpose (Allen and Tett, 1999). Ribes et al. (2013) raise some issues with this test, but key results are not found to be sensitive to different formulations. A more important issue is that the F-test is relatively weak (Berliner et al., 2000; Allen et al., 2006; Terray, 2012), so 'passing' this test is not a safeguard against unrealistic variability, which is why estimates of internal variability are discussed in detail in this chapter and in Chapter 9.

A further consistency check often used in optimal fingerprinting is whether the estimated magnitude of the externally driven responses are consistent between model and observations (scaling factors consistent with unity in Box 10.1): if they are not, attribution is still possible provided the discrepancy is explicable in terms of known uncertainties in the magnitude of either forcing or response. As is emphasized in Section 10.2.1 and Box 10.1, attribution is not a purely statistical assessment: physical judgment is required to assess whether the combination of responses considered allows for all major potential confounding factors and whether any remaining discrepancies are consistent with a physically based understanding of the responses to external forcing and internal climate variability.

\subsubsection{Single-Step and Multi-Step Attribution and the Role of the Null Hypothesis}

Attribution studies have traditionally involved explicit simulation of the response to external forcing of an observable variable, such as surface temperature, and comparison with corresponding observations of that variable. This so-called 'single-step attribution' has the advantage of simplicity, but restricts attention to variables for which long and consistent time series of observations are available and that can be simulated explicitly in current models driven solely with external climate forcing.

To address attribution questions for variables for which these conditions are not satisfied, Hegerl et al. (2010) introduced the notation of 'multi-step attribution', formalizing existing practice (e.g., Stott et al., 2004). In a multi-step attribution study, the attributable change in a variable such as large-scale surface temperature is estimated with a single-step procedure, along with its associated uncertainty, and the implications of this change are then explored in a further (physically or statistically based) modelling step. Overall conclusions can only be as robust as the least certain link in the multi-step procedure. As the focus shifts towards more noisy regional changes, it can be difficult to separate the effect of different external forcings. In such cases, it can be useful to detect the response to all external forcings, and then determine the most important factors underlying the attribution results by reference to a closely related variable for which a full attribution analysis is available (e.g., Morak et al., 2011).

Attribution results are typically expressed in terms of conventional 'frequentist' confidence intervals or results of hypothesis tests: when it is reported that the response to anthropogenic GHG increase is very likely greater than half the total observed warming, it means that the null hypothesis that the GHG-induced warming is less than half the total can be rejected with the data available at the $10 \%$ significance level. Expert judgment is required in frequentist attribution assessments, but its role is limited to the assessment of whether internal variability and potential confounding factors have been adequately accounted for, and to downgrade nominal significance levels to account for remaining uncertainties. Uncertainties may, in some cases, be further reduced if prior expectations regarding attribution results themselves are incorporated, using a Bayesian approach, but this not currently the usual practice.

This traditional emphasis on single-step studies and placing lower bounds on the magnitude of signals under investigation means that, very often, the communication of attribution results tends to be conservative, with attention focussing on whether or not human influence in a particular variable might be zero, rather than the upper end of the confidence interval, which might suggest a possible response much bigger than current model-simulated changes. Consistent with previous Assessments and the majority of the literature, this chapter adopts this conservative emphasis. It should, however, be borne in mind that this means that positive attribution results will tend to be biased towards well-observed, well-modelled variables and regions, which should be taken into account in the compilation of global impact assessments (Allen, 2011; Trenberth, 2011a).

\subsection{Atmosphere and Surface}

This section assesses causes of change in the atmosphere and at the surface over land and ocean.

\subsubsection{Temperature}

Temperature is first assessed near the surface of the Earth in Section 10.3.1.1 and then in the free atmosphere in Section 10.3.1.2.

\subsubsection{Surface (Air Temperature and Sea Surface Temperature)}

\subsection{Observations of surface temperature change}

GMST warmed strongly over the period 1900-1940, followed by a period with little trend, and strong warming since the mid-1970s (Section 2.4.3, Figure 10.1). Almost all observed locations have warmed since 1901 whereas over the satellite period since 1979 most regions have warmed while a few regions have cooled (Section 2.4.3; Figure 10.2). Although this picture is supported by all available global near-surface temperature data sets, there are some differences in detail between them, but these are much smaller than both interannual variability and the long-term trend (Section 2.4.3). Since 1998 the trend in GMST has been small (see Section 2.4.3, Box 9.2). Urbanization is unlikely to have caused more than $10 \%$ of the measured centennial trend in land mean surface temperature, though it may have contributed substantially more to regional mean surface temperature trends in rapidly developing regions (Section 2.4.1.3). 


\subsection{Simulations of surface temperature change}

As discussed in Section 10.1, the CMIP5 simulations have several advantages compared to the CMIP3 simulations assessed by (Hegerl et al., 2007b) for the detection and attribution of climate change. Figure 10.1a shows that when the effects of anthropogenic and natural external forcings are included in the CMIP5 simulations the spread of sim-
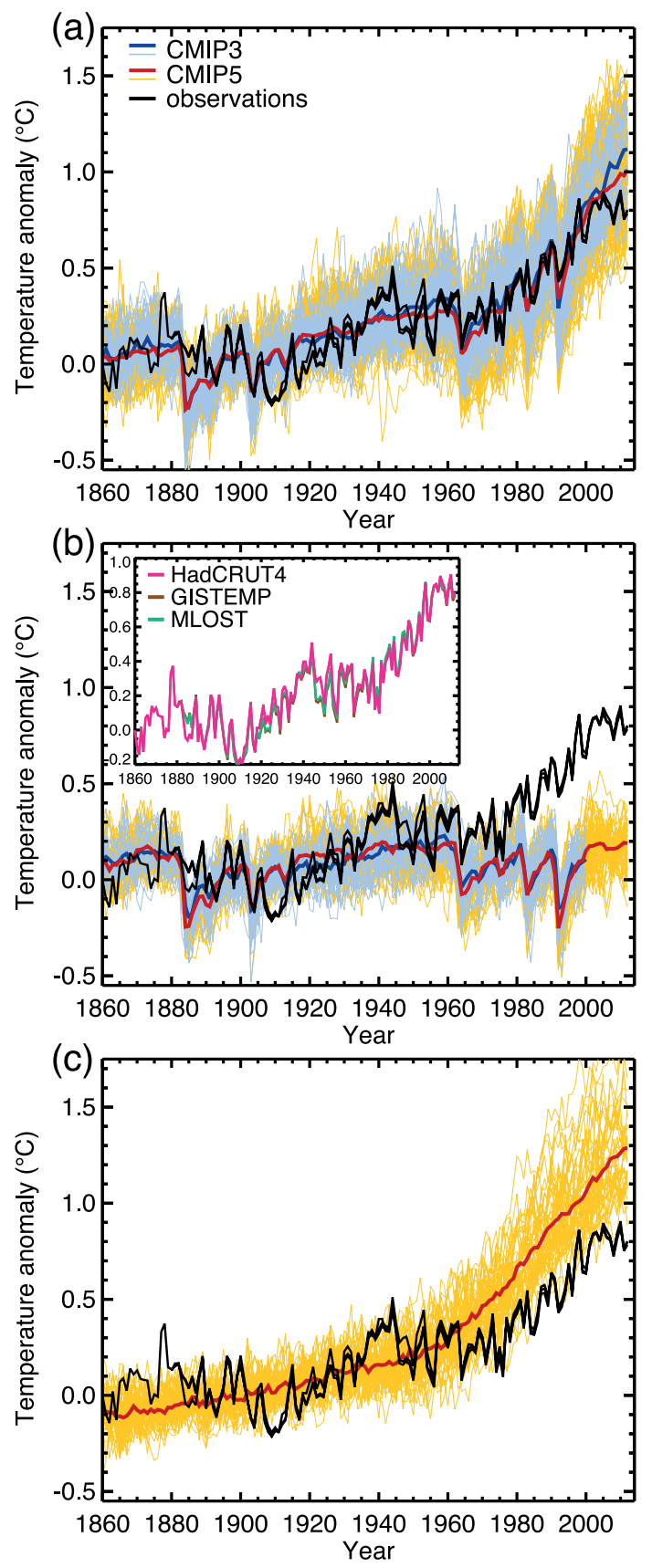

ulated GMST anomalies spans the observational estimates of GMST anomaly in almost every year whereas this is not the case for simulations in which only natural forcings are included (Figure 10.1b) (see also Jones et al., 2013; Knutson et al., 2013). Anomalies are shown relative to 1880-1919 rather than absolute temperatures. Showing anomalies is necessary to prevent changes in observational coverage being reflected in the calculated global mean and is reasonable
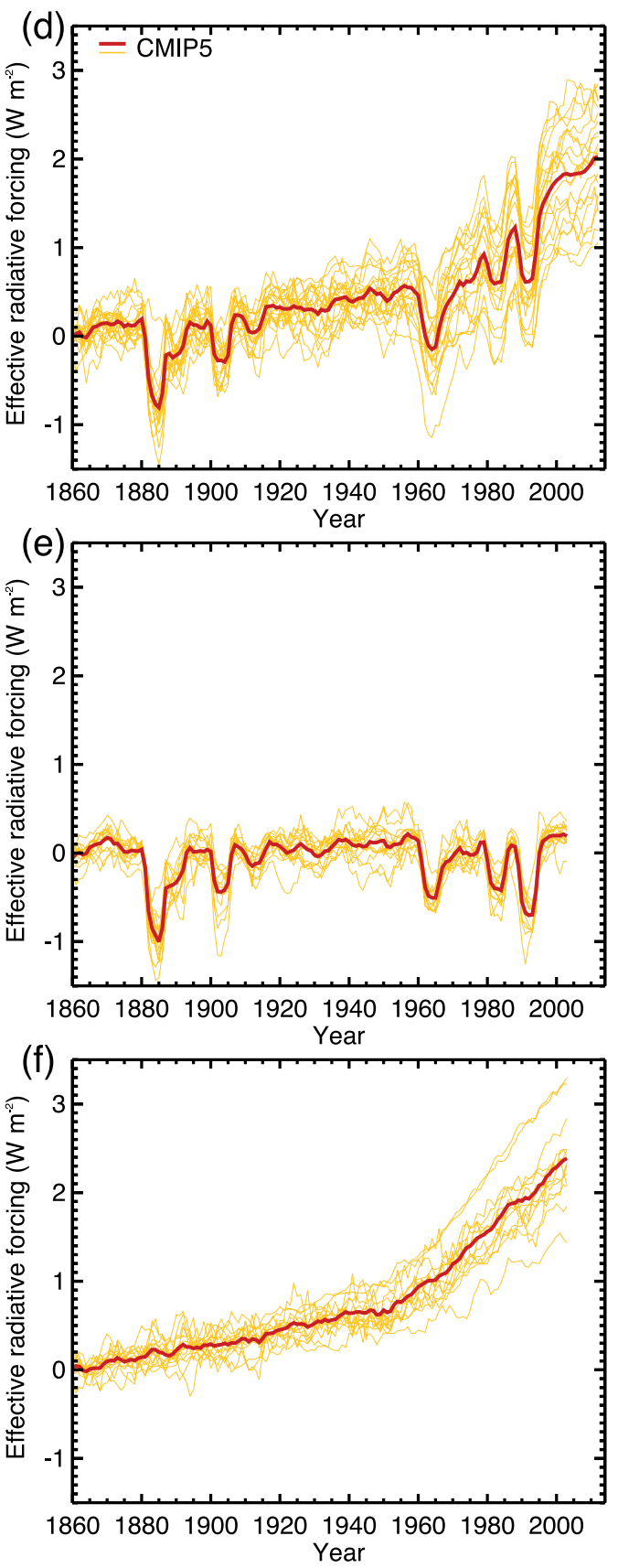

Figure 10.1 | (Left-hand column) Three observational estimates of global mean surface temperature (GMST, black lines) from Hadley Centre/Climatic Research Unit gridded surface temperature data set 4 (HadCRUT4), Goddard Institute of Space Studies Surface Temperature Analysis (GISTEMP), and Merged Land-Ocean Surface Temperature Analysis (MLOST), compared to model simulations [CMIP3 models - thin blue lines and CMIP5 models - thin yellow lines] with anthropogenic and natural forcings (a), natural forcings only (b) and greenhouse gas (GHG) forcing only (c). Thick red and blue lines are averages across all available CMIP5 and CMIP3 simulations respectively. CMIP3 simulations were not available for GHG forcing only (c). All simulated and observed data were masked using the HadCRUT4 coverage (as this data set has the most restricted spatial coverage), and global average anomalies are shown with respect to 1880-1919, where all data are first calculated as anomalies relative to 1961-1990 in each grid box. Inset to (b) shows the three observational data sets distinguished by different colours. (Adapted from Jones et al., 2013.) (Right-hand column) Net adjusted forcing in CMIP5 models due to anthropogenic and natural forcings (d), natural forcings only (e) and GHGs only (f). (From Forster et al., 2013.) Individual ensemble members are shown by thin yellow lines, and CMIP5 multi-model means are shown as thick red lines. 
because climate sensitivity is not a strong function of the bias in GMST in the CMIP5 models (Section 9.7.1; Figure 9.42). Simulations with GHG changes only, and no changes in aerosols or other forcings, tend to simulate more warming than observed (Figure 10.1c), as expected. Better agreement between models and observations when the models include anthropogenic forcings is also seen in the CMIP3 simulations (Figure 10.1, thin blue lines). RF in the simulations including anthropogenic and natural forcings differs considerably among models (Figure 10.1d), and forcing differences explain much of the differences in temperature response between models over the historical period (Forster et al., 2013 ). Differences between observed GMST based on three observational data sets are small compared to forced changes (Figure 10.1).

As discussed in Section 10.2, detection and attribution assessments are more robust if they consider more than simple consistency arguments. Analyses that allow for the possibility that models might be consistently over- or underestimating the magnitude of the response to climate forcings are assessed in Section 10.3.1.1.3, the conclusions from which are not affected by evidence that model spread in GMST in CMIP3, is smaller than implied by the uncertainty in RF (Schwartz et al., 2007). Although there is evidence that CMIP3 models with a higher climate sensitivity tend to have a smaller increase in RF over the historical period (Kiehl, 2007; Knutti, 2008; Huybers, 2010), no such relationship was found in CMIP5 (Forster et al., 2013 ) which may explain the wider spread of the CMIP5 ensemble compared to the CMIP3 ensemble (Figure 10.1a). Climate model parameters are typically chosen primarily to reproduce features of the mean climate and variability (Box 9.1), and CMIP5 aerosol emissions are standardized across models and based on historical emissions (Lamarque et al., 2010; Section 8.2.2), rather than being chosen by each modelling group independently (Curry and Webster, 2011; Hegerl et al., 2011c).

Figure 10.2a shows the pattern of annual mean surface temperature trends observed over the period 1901-2010, based on Hadley Centre/ Climatic Research Unit gridded surface temperature data set 4 ( HadCRUT4). Warming has been observed at almost all locations with sufficient observations available since 1901. Rates of warming are generally higher over land areas compared to oceans, as is also apparent over the 1951-2010 period (Figure 10.2c), which simulations indicate is due mainly to differences in local feedbacks and a net anomalous heat transport from oceans to land under GHG forcing, rather than differences in thermal inertia (e.g., Boer, 2011). Figure 10.2e demonstrates that a similar pattern of warming is simulated in the CMIP5 simulations with natural and anthropogenic forcing over the 1901-2010 period. Over most regions, observed trends fall between the 5th and 95th percentiles of simulated trends, and van Oldenborgh et al. (2013) find that over the 1950-2011 period the pattern of observed grid cell trends agrees with CMIP5 simulated trends to within a combination of

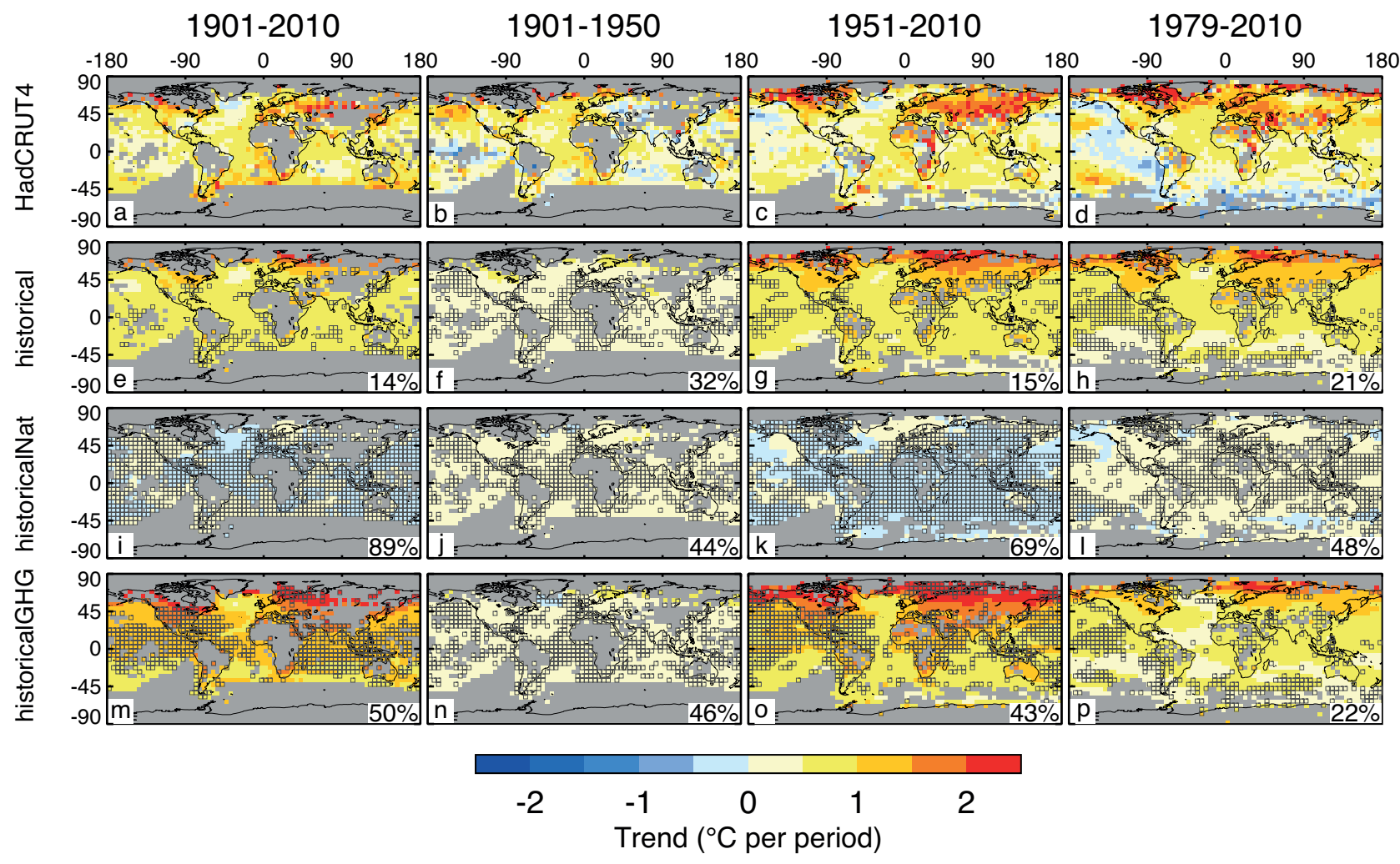

Figure 10.2 Trends in observed and simulated temperatures (K over the period shown) over the 1901-2010 (a, e, i, $m), 1901-1950(b, f, j, n), 1951-2010(c, g, k, 0)$ and 1979-2010 (d, h, l, p) periods. Trends in observed temperatures from the Hadley Centre/Climatic Research Unit gridded surface temperature data set 4 (HadCRUT4) (a-d), CMIP3 and CMIP5 model simulations including anthropogenic and natural forcings (e-h), CMIP3 and CMIP5 model simulations including natural forcings only (i-I) and CMIP3 and CMIP5 model simulations including greenhouse gas forcing only $(\mathrm{m}-\mathrm{p})$. Trends are shown only where sufficient observational data are available in the HadCRUT4 data set, and grid cells with insufficient observations to derive trends are shown in grey. Boxes in (e-p) show where the observed trend lies outside the 5 to 95 th percentile range of simulated trends, and the ratio of the number of such grid cells to the total number of grid cells with sufficient data is shown as a percentage in the lower right of each panel. (Adapted from Jones et al., 2013.) 
model spread and internal variability. Areas of disagreement over the 1901-2010 period include parts of Asia and the Southern Hemisphere (SH) mid-latitudes, where the simulations warm less than the observations, and parts of the tropical Pacific, where the simulations warm more than the observations (Jones et al., 2013; Knutson et al., 2013). Stronger warming in observations than models over parts of East Asia could in part be explained by uncorrected urbanization influence in the observations (Section 2.4.1.3), or by an overestimate of the response to aerosol increases. Trends simulated in response to natural forcings only are generally close to zero, and inconsistent with observed trends in most locations (Figure 10.2i) (see also Knutson et al., 2013). Trends simulated in response to GHG changes only over the 1901-2010 period are larger than those observed at most locations, and in many cases significantly so (Figure $10.2 \mathrm{~m}$ ). This is expected because these simulations do not include the cooling effects of aerosols. Differences in patterns of simulated and observed seasonal mean temperature trends and possible causes are considered in more detail in Box 11.2.

Over the period 1979-2010 most observed regions exhibited warming (Figure 10.2d), but much of the eastern Pacific and Southern Oceans cooled. These regions of cooling are not seen in the simulated trends over this period in response to anthropogenic and natural forcing (Figure 10.2h), which show significantly more warming in much of these regions (Jones et al., 2013; Knutson et al., 2013). This cooling and reduced warming in observations over the Southern Hemisphere mid-latitudes over the 1979-2010 period can also be seen in the zonal mean trends (Figure 10.3d), which also shows that the models tend to warm too much in this region over this period. However, there is no discrepancy in zonal mean temperature trends over the longer 1901-2010 period in this region (Figure 10.3a), suggesting that the discrepancy over the 1979-2010 period either may be an unusually strong manifestation of internal variability in the observations or relate to regionally important forcings over the past three decades which are not included in most CMIP5 simulations, such as sea salt aerosol increases due to strengthened high latitude winds (Korhonen et al., 2010), or sea ice extent increases driven by freshwater input from ice shelf melting (Bintanja et al., 2013). Except at high latitudes, zonal mean trends over the 1901-2010 period in all three data sets are inconsistent with naturally forced trends, indicating a detectable anthropogenic signal in most zonal means over this period (Figure 10.3a). McKitrick and Tole (2012) find that few CMIP3 models have significant explanatory power when fitting the spatial pattern of 1979-2002 trends in surface temperature over land, by which they mean that these models add little or no skill to a fit including the spatial pattern of tropospheric temperature trends as well as the major atmospheric oscillations. This is to be expected, as temperatures in the troposphere are well correlated in the vertical, and local temperature trends over so short a period are dominated by internal variability.

CMIP5 models generally exhibit realistic variability in GMST on decadal to multi-decadal time scales (Jones et al., 2013; Knutson et al., 2013; Section 9.5.3.1, Figure 9.33), although it is difficult to evaluate internal variability on multi-decadal time scales in observations given the shortness of the observational record and the presence of external forcing. The observed trend in GMST since the 1950s is very large compared to model estimates of internal variability (Stott et al., 2010; Drost et al., 2012; Drost and Karoly, 2012). Knutson et al. (2013) compare observed

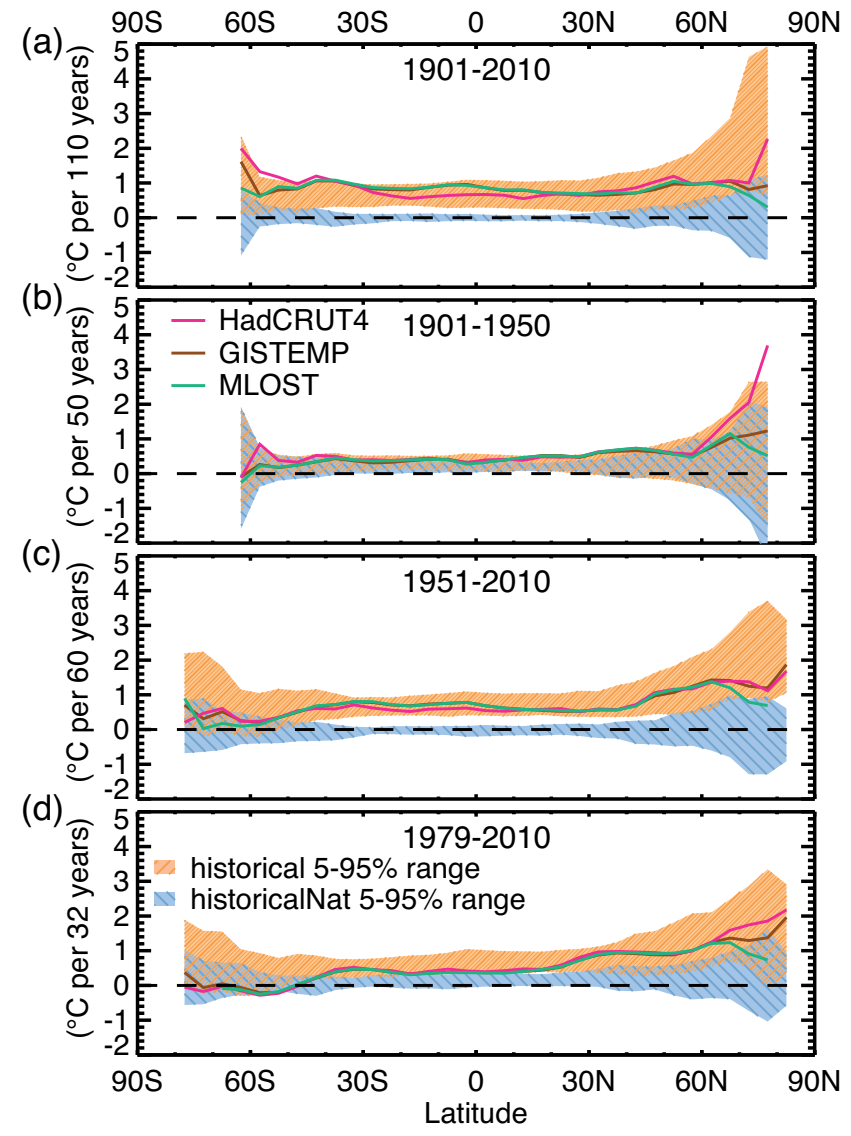

Figure 10.3 | Zonal mean temperature trends over the 1901-2010 (a), 1901-1950 (b), 1951-2010 (c) and 1979-2010 (d) periods. Solid lines show Hadley Centre/Climatic Research Unit gridded surface temperature data set 4 (HadCRUT4, red), Goddard Institute of Space Studies Surface Temperature Analysis (GISTEMP, brown) and Merged Land-Ocean Surface Temperature Analysis (MLOST, green) observational data sets, orange hatching represents the $90 \%$ central range of CMIP3 and CMIP5 simulations with anthropogenic and natural forcings, and blue hatching represents the $90 \%$ central range of CMIP3 and CMIP5 simulations with natural forcings only. All model and observations data are masked to have the same coverage as HadCRUT4. (Adapted from Jones et al., 2013.)

trends in GMST with a combination of simulated internal variability and the response to natural forcings and find that the observed trend would still be detected for trends over this period even if the magnitude of the simulated natural variability (i.e., the standard deviation of trends) were tripled.

\subsection{Attribution of observed global-scale temperature changes}

\section{The evolution of temperature since the start of the global instrumental record}

Since the AR4, detection and attribution studies have been carried out using new model simulations with more realistic forcings, and new observational data sets with improved representation of uncertainty (Christidis et al., 2010; Jones et al., 2011, 2013; Gillett et al., 2012, 2013; Stott and Jones, 2012; Knutson et al., 2013; Ribes and Terray, 2013). Although some inconsistencies between the simulated and observed responses to forcings in individual models were identified ( Gillett et al., 2013; Jones et al., 2013; Ribes and Terray, 2013) over- 
all these results support the AR4 assessment that GHG increases very likely caused most ( $>50 \%$ ) of the observed GMST increase since the mid-20th century (Hegerl et al., 2007b).

The results of multiple regression analyses of observed temperature changes onto the simulated responses to GHG, other anthropogenic and natural forcings are shown in Figure 10.4 (Gillett et al., 2013; Jones et al., 2013; Ribes and Terray, 2013). The results, based on HadCRUT4 and a multi-model average, show robustly detected responses to GHG in the observational record whether data from 1861-2010 or only from 1951-2010 are analysed (Figure 10.4b). The advantage of analysing the longer period is that more information on observed and modelled changes is included, while a disadvantage is that it is difficult to validate climate models' estimates of internal variability over such a long period. Individual model results exhibit considerable spread among scaling factors, with estimates of warming attributable to each forcing sensitive to the model used for the analsys (Figure 10.4; Gillett et al., 2013; Jones et al., 2013; Ribes and Terray, 2013), the period over which the analysis is applied (Figure 10.4; Gillett et al., 2013; Jones et al., 2013), and the Empirical Orthogonal Function (EOF) truncation or degree of spatial filtering (Jones et al., 2013; Ribes and Terray, 2013). In some cases the GHG response is not detectable in regressions using individual models (Figure 10.4; Gillett et al., 2013; Jones et al., 2013; Ribes and Terray, 2013), or a residual test is failed (Gillett et al., 2013; Jones et al., 2013; Ribes and Terray, 2013), indicating a poor fit between the simulated response and observed changes. Such cases are probably due largely to errors in the spatio-temporal pattern of responses to forcings simulated in individual models (Ribes and Terray, 2013), although observational error and internal variability errors could also play a role. Nonetheless, analyses in which responses are averaged across multiple models generally show much less sensitivity to period and EOF trucation (Gillett et al., 2013; Jones et al., 2013), and more consistent residuals (Gillett et al., 2013), which may be because model response errors are smaller in a multi-model mean.

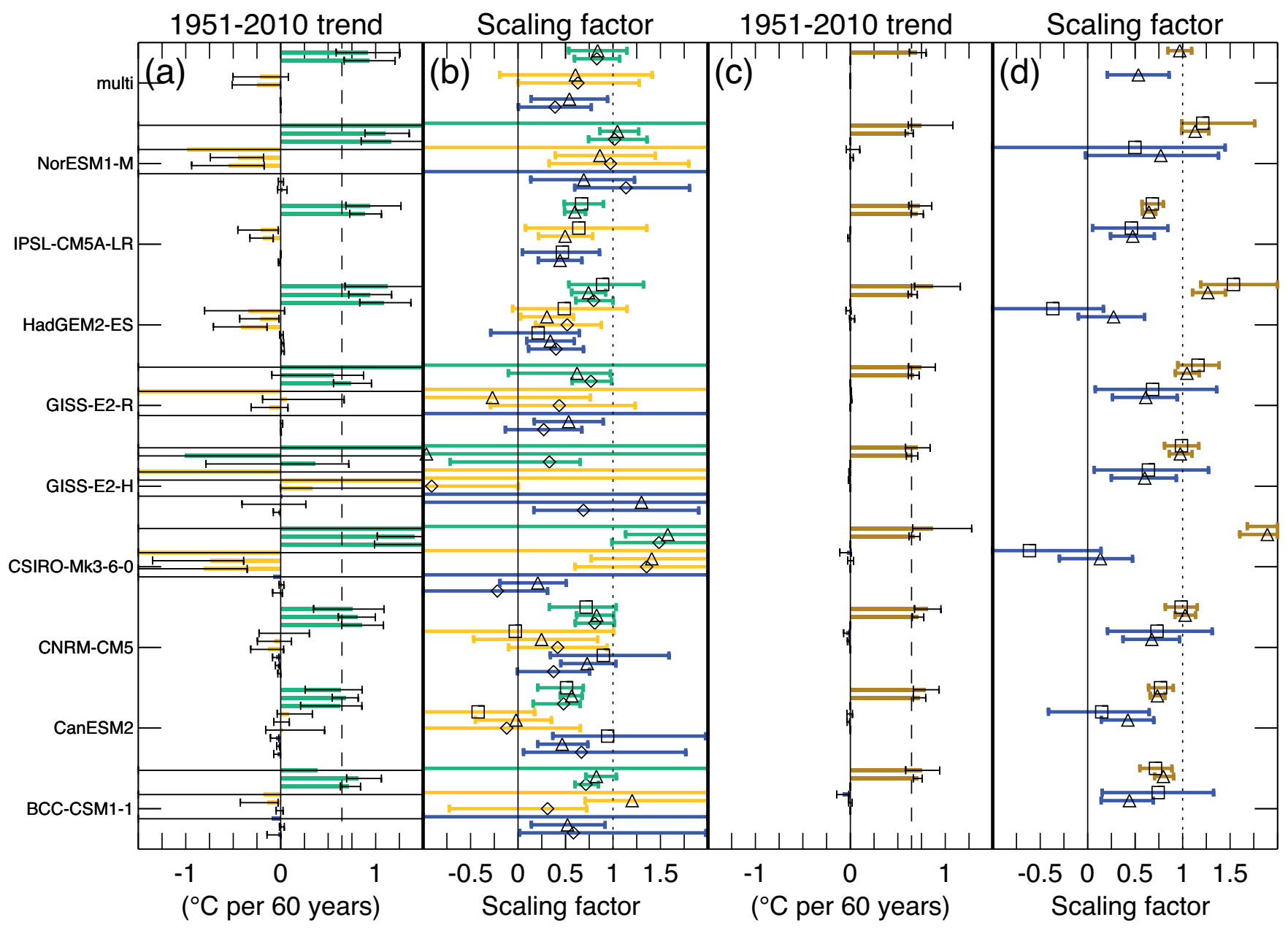

Figure 10.4 | (a) Estimated contributions of greenhouse gas (GHG, green), other anthropogenic (yellow) and natural (blue) forcing components to observed global mean surface temperature (GMST) changes over the 1951-2010 period. (b) Corresponding scaling factors by which simulated responses to GHG (green), other anthropogenic (yellow) and natural forcings (blue) must be multiplied to obtain the best fit to Hadley Centre/Climatic Research Unit gridded surface temperature data set 4 (HadCRUT4; Morice et al., 2012) observations based on multiple regressions using response patterns from nine climate models individually and multi-model averages (multi). Results are shown based on an analysis over the 1901-2010 period (squares, Ribes and Terray, 2013), an analysis over the 1861-2010 period (triangles, Gillett et al., 2013) and an analysis over the 1951-2010 period (diamonds, Jones et al., 2013). (c, d) As for (a) and (b) but based on multiple regressions estimating the contributions of total anthropogenic forcings (brown) and natural forcings (blue) based on an analysis over 1901-2010 period (squares, Ribes and Terray, 2013) and an analysis over the 1861-2010 period (triangles, Gillett et al., 2013). Coloured bars show best estimates of the attributable trends ( $a$ and $c$ ) and 5 to $95 \%$ confidence ranges of scaling factors ( $b$ and $d)$. Vertical dashed lines in (a) and (c) show the best estimate HadCRUT4 observed trend over the period concerned. Vertical dotted lines in (b) and d) denote a scaling factor of unity. 
We derive assessed ranges for the attributable contributions of GHGs, other anthropogenic forcings and natural forcings by taking the smallest ranges with a precision of one decimal place that span the 5 to 95\% ranges of attributable trends over the 1951-2010 period from the Jones et al. (2013) weighted multi-model analysis and the Gillett et al. (2013) multi-model analysis considering observational uncertainty (Figure 10.4a). The assessed range for the attributable contribution of combined anthropogenic forcings was derived in the same way from the Gillett et al. (2013) multi-model attributable trend and shown in Figure 10.4c. We moderate our likelihood assessment and report likely ranges rather than the very likely ranges directly implied by these studies in order to account for residual sources of uncertainty including sensitivity to EOF truncation and analysis period (e.g., Ribes and Terray, 2013). In this context, GHGs means well-mixed greenhouse gases (WMGHGs), other anthropogenic forcings means aerosol changes, and in most models ozone changes and land use changes, and natural forcings means solar irradiance changes and volcanic aerosols. Over the 1951-2010 period, the observed GMST increased by approximately $0.6^{\circ} \mathrm{C}$. GHG increases likely contributed $0.5^{\circ} \mathrm{C}$ to $1.3^{\circ} \mathrm{C}$, other anthropogenic forcings likely contributed $-0.6^{\circ} \mathrm{C}$ to $0.1^{\circ} \mathrm{C}$ and natural forcings likely contributed $-0.1^{\circ} \mathrm{C}$ to $0.1^{\circ} \mathrm{C}$ to observed GMST trends over this period. Internal variability likely contributed $-0.1^{\circ} \mathrm{C}$ to $0.1^{\circ} \mathrm{C}$ to observed trends over this period (Knutson et al., 2013). This assessment is shown schematically in Figure 10.5. The assessment is supported additionally by a complementary analysis in which the parameters of an Earth System Model of Intermediate Complexity (EMIC) were constrained using observations of near-surface temperature and ocean heat content, as well as prior information on the magnitudes of forcings, and which concluded that GHGs have caused $0.6^{\circ} \mathrm{C}$ to $1.1^{\circ} \mathrm{C}(5$ to $95 \%$ uncertainty) warming since the mid-20th century (Huber and Knutti, 2011); an analysis by Wigley and Santer (2013), who used an energy balance model and RF and climate sensitivity estimates from AR4, and they concluded that there was about a 93\% chance that GHGs caused a warming greater than observed over the 1950-2005 period; and earlier detection and attribution studies assessed in the AR4 (Hegerl et al., 2007b).

The inclusion of additional data to 2010 (AR4 analyses stopped at 1999; Hegerl et al. (2007b)) helps to better constrain the magnitude of the GHG-attributable warming (Drost et al., 2012; Gillett et al., 2012; Stott and Jones, 2012; Gillett et al., 2013), as does the inclusion of spatial information (Stott et al., 2006; Gillett et al., 2013), though Ribes and Terray (2013) caution that in some cases there are inconsistencies between observed spatial patterns of response and those simulated in indvidual models. While Hegerl et al. (2007b) assessed that a significant cooling of about $0.2{ }^{\circ} \mathrm{C}$ was attributable to natural forcings over the 1950-1999 period, the temperature trend attributable to natural forcings over the $1951-2010$ period is very small $\left(<0.1^{\circ} \mathrm{C}\right)$. This is because, while Mt Pinatubo cooled global temperatures in the early 1990s, there have been no large volcanic eruptions since, resulting in small simulated trends in response to natural forcings over the 1951-2010 period (Figure 10.1b). Regression coefficients for natural forcings tend to be smaller than one, suggesting that the response to natural forcings may be overestimated by the CMIP5 models on average (Figure 10.4; Gillett et al., 2013; Knutson et al., 2013). Attribution of observed changes is robust to observational uncertainty which is comparably important to internal climate variability as a source of uncertainty in
GHG-attributable warming and aerosol-attributable cooling (Jones and Stott, 2011; Gillett et al., 2013; Knutson et al., 2013). The response to GHGs was detected using Hadley Centre new Global Environmental Model 2-Earth System (HadGEM2-ES; Stott and Jones, 2012), Canadian Earth System Model 2 (CanESM2; Gillett et al., 2012) and other CMIP5 models except for Goddard Institute for Space Studies-E2-H (GISSE2-H; Gillett et al., 2013; Jones et al., 2013) (Figure 10.4). However, the influence of other anthropogenic forcings was detected only in some CMIP5 models (Figure 10.4). This lack of detection of other anthropogenic forcings compared to detection of an aerosol response using four CMIP3 models over the period 1900-1999 (Hegerl et al., 2007b) does not only relate to the use of data to 2010 rather than 2000 (Stott and Jones, 2012), although this could play a role (Gillett et al., 2013; Ribes and Terray, 2013). Whether it is associated with a cancellation of aerosol cooling by ozone and black carbon (BC) warming in the CMIP5 simulations, making the signal harder to detect, or by some aspect of the response to other anthropogenic forcings that is less realistic in these models is not clear.

Although closely constraining the GHG and other anthropogenic contributions to observed warming remains challenging owing to their degeneracy and sensitivity to methodological choices (Jones et al., 2013; Ribes and Terray, 2013), a total anthropogenic contribution to warming can be much more robustly constrained by a regression of observed temperature changes onto the simulated responses to all anthropogenic forcings and natural forcings (Figure 10.4; Gillett et al., 2013; Ribes and Terray, 2013). Robust detection of anthropogenic influence is also found if a new optimal detection methodology, the Regularised Optimal Fingerprint approach (see Section 10.2; Ribes et al., 2013), is applied (Ribes and Terray, 2013). A better constrained estimate of the total anthropogenic contribution to warming since the mid-20th century than the GHG contribution is also found by Wigley and Santer (2013). Knutson et al. (2013) demonstrate that observed trends in GMST are inconsistent with the simulated response to natural forcings alone, but consistent with the simulated response to natural and anthropogenic forcings for all periods beginning between 1880 and 1990 and ending in 2010, which they interpret as evidence that warming is in part attributable to anthropogenic influence over these periods. Based on the well-constrained attributable anthropogenic trends shown in Figure 10.4 we assess that anthropogenic forcings likely contributed $0.6^{\circ} \mathrm{C}$ to $0.8^{\circ} \mathrm{C}$ to the observed warming over the 1951-2010 period (Figure 10.5).

There are some inconsistencies in the simulated and observed magnitudes of responses to forcing for some CMIP5 models (Figure 10.4); for example, CanESM2 has a GHG regression coefficient significantly less than 1 and a regression coefficient for other anthropogenic forcings also significantly less than 1 (Gillett et al., 2012; Gillett et al., 2013; Jones et al., 2013; Ribes and Terray, 2013), indicating that this model overestimates the magnitude of the response to GHGs and to other anthropogenic forcings. Averaged over the ensembles of models considered by Gillett et al. (2013) and Jones et al. (2013), the best-estimate GHG and OA scaling factors are less than 1 (Figure 10.4), indicating that the model mean GHG and OA responses should be scaled down to best match observations. The best-estimate GHG scaling factors are larger than the best-estimate OA scaling factors, although the discrepancy from 1 is not significant in either case and the ranges of the GHG 


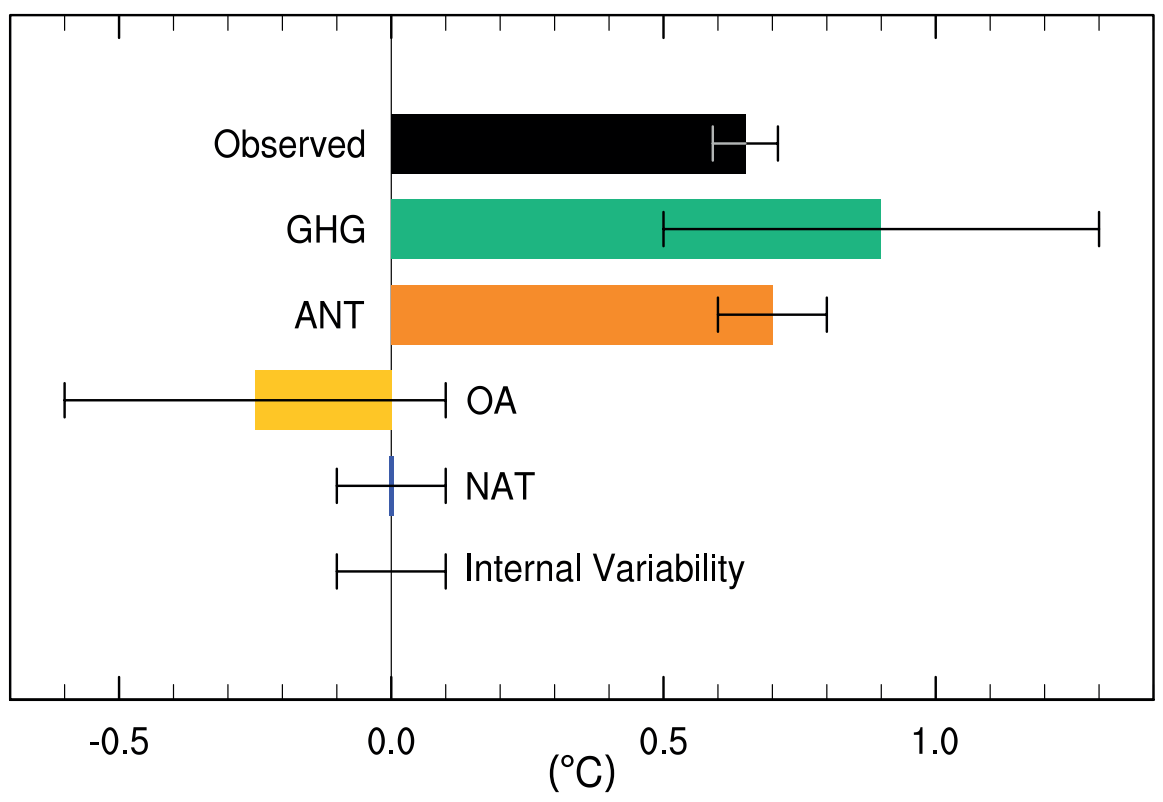

Figure 10.5 | Assessed likely ranges (whiskers) and their mid-points (bars) for attributable warming trends over the 1951-2010 period due to well-mixed greenhouse gases, other anthropogenic forcings (OA), natural forcings (NAT), combined anthropogenic forcings (ANT) and internal variability. The Hadley Centre/Climatic Research Unit gridded surface temperature data set 4 (HadCRUT4) observations are shown in black with the 5 to $95 \%$ uncertainty range due to observational uncertainty in this record (Morice et al., 2012).

and OA scaling factors are overlapping. Overall there is some evidence that some CMIP5 models have a higher transient response to GHGs and a larger response to other anthropogenic forcings (dominated by the effects of aerosols) than the real world (medium confidence). Inconsistencies between simulated and observed trends in GMST were also identified in several CMIP3 models by Fyfe et al. (2010) after removing volcanic, El Niño-Southern Oscillation (ENSO), and Cold Ocean/ Warm Land pattern (COWL) signals from GMST, although uncertainties may have been underestimated because residuals were modelled by a first-order autoregressive processes. A longer observational record and a better understanding of the temporal changes in forcing should make it easier to identify discrepancies between the magnitude of the observed response to a forcing, and the magnitude of the response simulated in individual models. To the extent that inconsistencies between simulated and observed changes are independent between models, this issue may be addressed by basing our assessment on attribution analyses using the mean response from multiple models, and by accounting for model uncertainty when making such assessments.

In conclusion, although some inconsistencies in the forced responses of individual models and observations have been identified, the detection of the global temperature response to GHG increases using average responses from multiple models is robust to observational uncertainty and methodological choices. It is supported by basic physical arguments. We conclude, consistent with Hegerl et al. (2007b), that more than half of the observed increase in GMST from 1951 to 2010 is very likely due to the observed anthropogenic increase in GHG concentrations.

The influence of BC aerosols (from fossil and biofuel sources) has been detected in the recent global temperature record in one analysis, although the warming attributable to BC by Jones et al. (2011) is small compared to that attributable to GHG increases. This warming is simulated mainly over the Northern Hemisphere (NH) with a sufficiently distinct spatio-temporal pattern that it could be separated from the response to other forcings in this study.

Several recent studies have used techniques other than regression-based detection and attribution analyses to address the causes of recent global temperature changes. Drost and Karoly (2012) demonstrated that observed GMST, land-ocean temperature contrast, meridional temperature gradient and annual cycle amplitude exhibited trends over the period 1956-2005 that were outside the 5 to $95 \%$ range of simulated internal variability in eight CMIP5 models, based on three different observational data sets. They also found that observed trends in GMST and land-ocean temperature contrast were larger than those simulated in any of 36 CMIP5 simulations with natural forcing only. Drost et al. (2012) found that 1961-2010 trends in GMST and land-ocean temperature contrast were significantly larger than simulated internal variability in eight CMIP3 models. By comparing observed GMST with simple statistical models, Zorita et al. (2008) concluded that there is a very low probability that observed clustering of very warm years in the last decade occurred by chance. Smirnov and Mokhov (2009), adopting an approach that allowed them to distinguish between conventional Granger causality and a 'long-term causality' that focuses on low-frequency changes (see Section 10.2), found that increasing $\mathrm{CO}_{2}$ concentrations are the principal determining factor in the rise of GMST over recent decades. Sedlacek and Knutti (2012) found that the spatial patterns of sea surface temperature (SST) trends from simulations forced with increases in GHGs and other anthropogenic forcings agree well with observations but differ from warming patterns associated with internal variability.

Several studies that have aimed to separate forced surface temperature variations from those associated with internal variability have identified the North Atlantic as a dominant centre of multi-decadal 
internal variability, and in particular modes of variability related to the Atlantic Multi-decadal Oscillation (AMO; Section 14.7.6). The AMO index is defined as an area average of North Atlantic SSTs, and it has an apparent period of around 70 years, which is long compared to the length of observational record making it difficult to deduce robust conclusions about the role of the AMO from only two cycles. Nevertheless, several studies claim a role for internal variability associated with the AMO in driving enhanced warming in the 1980s and 1990s as well as the recent slow down in warming (Box 9.2), while attributing long-term warming to anthropogenically forced variations either by analysing time series of GMST, forcings and indices of the AMO (Rohde et al., 2013; Tung and Zhou, 2013; Zhou and Tung, 2013a) or by analysing both spatial and temporal patterns of temperature (Swanson et al., 2009; DelSole et al., 2011; Wu et al., 2011). Studies based on global mean time series could risk falsely attributing variability to the AMO when variations in external forcings, for example, associated with aerosols, could also cause similar variability. In contrast, studies using space-time patterns seek to distinguish the spatial structure of temperature anomalies associated with the AMO from those associated with forced variability. Unforced climate simulations indicate that internal multi-decadal variability in the Atlantic is characterized by surface anomalies of the same sign from the equator to the high latitudes, with maximum amplitudes in subpolar regions (Delworth and Mann, 2000; Latif et al., 2004; Knight et al., 2005; DelSole et al., 2011) while the net response to anthropogenic and natural forcing over the 20th century, such as observed temperature change, is characterized by warming nearly everywhere on the globe, but with minimum warming or even cooling in the subpolar regions of the North Atlantic (Figure 10.2; Ting et al., 2009; DelSole et al., 2011).
Some studies implicate tropospheric aerosols in driving decadal variations in Atlantic SST (Evan et al., 2011; Booth et al., 2012; Terray, 2012), and temperature variations in eastern North America (Leibensperger et al., 2012). Booth et al. (2012) find that most multi-decadal variability in North Atlantic SSTs is simulated in one model mainly in response to aerosol variations, although its simulated changes in North Atlantic ocean heat content and salinity have been shown to be inconsistent with observations (Zhang et al., 2012). To the extent that climate models simulate realistic internal variability in the AMO (Section 9.5.3.3.2), AMO variability is accounted for in uncertainty estimates from regression-based detection and attribution studies (e.g., Figure 10.4).

To summarize, recent studies using spatial features of observed temperature variations to separate AMO variability from externally forced changes find that detection of external influence on global temperatures is not compromised by accounting for AMO-congruent variability (high confidence). There remains some uncertainty about how much decadal variability of GMST that is attributed to AMO in some studies is actually related to forcing, notably from aerosols. There is agreement among studies that the contribution of the AMO to global warming since 1951 is very small (considerably less than $0.1^{\circ} \mathrm{C}$; see also Figure 10.6) and given that observed warming since 1951 is very large compared to climate model estimates of internal variability (Section 10.3.1.1.2), which are assessed to be adequate at global scale (Section 9.5.3.1), we conclude that it is virtually certain that internal variability alone cannot account for the observed global warming since 1951.

\section{Box 10.2 | The Sun's Influence on the Earth's Climate}

A number of studies since AR4 have addressed the possible influences of long-term fluctuations of solar irradiance on past climates, particularly related to the relative warmth of the Medieval Climate Anomaly (MCA) and the relative coolness in the Little Ice Age (LIA). There is medium confidence that both external solar and volcanic forcing, and internal variability, contributed substantially to the spatial patterns of surface temperature changes between the MCA and the LIA, but very low confidence in quantitative estimates of their relative contributions (Sections 5.3.5.3 and 5.5.1). The combined influence of volcanism, solar forcing and a small drop in greenhouse gases (GHGs) likely contributed to Northern Hemisphere cooling during the LIA (Section 10.7.2). Solar radiative forcing (RF) from the Maunder Minimum (1745) to the satellite era (average of 1976-2006) has been estimated to be +0.08 to $+0.18 \mathrm{~W} \mathrm{~m}^{-2}$ (low confidence, Section 8.4.1.2). This may have contributed to early 20th century warming (Iow confidence, Section 10.3.1).

More recently, it is extremely unlikely that the contribution from solar forcing to the observed global warming since 1950 was larger than that from GHGs (Section 10.3.1.1.3). It is very likely that there has been a small decrease in solar forcing of -0.04 [ -0.08 to 0.00 ] $\mathrm{W} \mathrm{m} \mathrm{m}^{-2}$ over a period with direct satellite measurements of solar output from 1986 to 2008 (Section 8.4.1.1). There is high confidence that changes in total solar irradiance have not contributed to global warming during that period.

Since AR4, there has been considerable new research that has connected solar forcing to climate. The effect of solar forcing on GMST trends has been found to be small, with less than $0.1^{\circ} \mathrm{C}$ warming attributable to combined solar and volcanic forcing over the $1951-$ 2010 period (Section 10.3.1), although the 11-year cycle of solar variability has been found to have some influence on GMST variability over the 20th century. GMST changes between solar maxima and minima are estimated to be of order $0.1^{\circ} \mathrm{C}$ from some regression studies of GMST and forcing estimates (Figure 10.6), although several studies have suggested these results may be too large owing to issues including degeneracy between forcing and with internal variability, overfitting of forcing indices and underestimated uncertainties in responses (Ingram, 2007; Benestad and Schmidt, 2009; Stott and Jones, 2009). Climate models generally show less than half this variability (Jones et al., 2012). (continued on next page) 
Variability associated with the 11-year solar cycle has also been shown to produce measurable short-term regional and seasonal climate anomalies (Miyazaki and Yasunari, 2008; Gray et al., 2010; Lockwood, 2012; National Research Council, 2012) particularly in the Indo-Pacific, Northern Asia and North Atlantic regions (medium evidence). For example, studies have suggested an 11-year solar response in the Indo-Pacific region in which the equatorial eastern Pacific sea surface temperatures (SSTs) tend to be below normal, the sea level pressure (SLP) in the Gulf of Alaska and the South Pacific above normal, and the tropical convergence zones on both hemispheres strengthened and displaced polewards under solar maximum conditions, although it can be difficult to discriminate the solar-forced signal from the El Niño-Southern Oscillation (ENSO) signal (van Loon et al., 2007; van Loon and Meehl, 2008; White and Liu, 2008; Meehl and Arblaster, 2009; Roy and Haigh, 2010, 2012; Tung and Zhou, 2010; Bal et al., 2011; Haam and Tung, 2012; Hood and Soukharev, 2012; Misios and Schmidt, 2012). For northern summer, there is evidence that for peaks in the 11-year solar cycle, the Indian monsoon is intensified (Kodera, 2004; van Loon and Meehl, 2012), with solar variability affecting interannual connections between the Indian and Pacific sectors due to a shift in the location of the descending branch of the Walker Circulation (Kodera et al., 2007). In addition, model sensitivity experiments (Ineson et al., 2011) suggest that the negative phase of the North Atlantic Oscillation (NAO) is more prevalent during solar minima and there is some evidence of this in observations, including an indication of increased frequency of high-pressure 'blocking' events over Europe in winter (Barriopedro et al., 2008; Lockwood et al., 2010; Woollings et al., 2010).

Two mechanisms have been identified in observations and simulated with climate models that could explain these low amplitude regional responses (Gray et al., 2010; medium evidence). These mechanisms are additive and may reinforce one another so that the response to an initial small change in solar irradiance is amplified regionally (Meehl et al., 2009). The first mechanism is a top-down mechanism first noted by Haigh (1996) where greater solar ultraviolet radiation (UV) in peak solar years warms the stratosphere directly via increased radiation and indirectly via increased ozone production. This can result in a chain of processes that influences deep tropical convection (Balachandran et al., 1999; Shindell et al., 1999; Kodera and Kuroda, 2002; Haigh et al., 2005; Kodera, 2006; Matthes et al., 2006). In addition, there is less heating than average in the tropical upper stratosphere under solar minimum conditions which weakens the equator-to-pole temperature gradient. This signal can propagate downward to weaken the tropospheric mid-latitude westerlies, thus favoring a negative phase of the Arctic Oscillation (AO) or NAO. This response has been shown in several models (e.g., Shindell et al., 2001; Ineson et al., 2011) though there is no significant AO or NAO response to solar irradiance variations on average in the CMIP5 models (Gillett and Fyfe, 2013).

The second mechanism is a bottom-up mechanism that involves coupled air-sea radiative processes in the tropical and subtropical Pacific that also influence convection in the deep tropics (Meehl et al., 2003, 2008; Rind et al., 2008; Bal et al., 2011; Cai and Tung, 2012; Zhou and Tung, 2013b). Such mechanisms have also been shown to influence regional temperatures over longer time scales (decades to centuries), and can help explain patterns of regional temperature changes seen in paleoclimate data (e.g., Section 10.7.2; Mann et al., 2009; Goosse et al., 2012b) although they have little effect on global or hemispheric mean temperatures at either short or long time scales.

A possible amplifying mechanism linking solar variability and the Earth's climate system via cosmic rays has been postulated. It is proposed that variations in the cosmic ray flux associated with changes in solar magnetic activity affect ion-induced aerosol nucleation and cloud condensation nuclei (CCN) production in the troposphere (Section 7.4.6). A strong solar magnetic field would deflect cosmic rays and lead to fewer CCN and less cloudiness, thereby allowing for more solar energy into the system. Since AR4, there has been further evidence to disprove the importance of this amplifying mechanism. Correlations between cosmic ray flux and observed aerosol or cloud properties are weak and local at best, and do not prove to be robust on the regional or global scale (Section 7.4.6). Although there is some evidence that ionization from cosmic rays may enhance aerosol nucleation in the free troposphere, there is medium evidence and high agreement that the cosmic ray-ionization mechanism is too weak to influence global concentrations of CCN or their change over the last century or during a solar cycle in any climatically significant way (Sections 7.4.6 and 8.4.1.5). The lack of trend in cosmic ray intensity over the 1960-2005 period (McCracken and Beer, 2007) provides another argument against the hypothesis of a major contribution of cosmic ray variations to the observed warming over that period given the existence of short time scales in the climate system response.

Thus, although there is medium confidence that solar variability has made contributions to past climate fluctuations, since the mid20th century there has been little trend in solar forcing. There are at least two amplifying mechanisms that have been proposed and simulated in some models that could explain small observed regional and seasonal climate anomalies associated with the 11-year solar cycle, mostly in the Indo-Pacific region and northern mid to high latitudes.

Regarding possible future influences of the sun on the Earth's climate, there is very low confidence in our ability to predict future solar output, but there is high confidence that the effects from solar irradiance variations will be much smaller than the projected climate changes from increased RF due to GHGs (Sections 8.4.1.3 and 11.3.6.2.2). 
Based on a range of detection and attribution analyses using multiple solar irradiance reconstructions and models, Hegerl et al. (2007b) concluded that it is very likely that GHGs caused more global warming than solar irradiance variations over the 1950-1999 period. Detection and attribution analyses applied to the CMIP5 simulations (Figure 10.4) indicate less than $0.1^{\circ} \mathrm{C}$ temperature change attributable to combined solar and volcanic forcing over the 1951-2010 period. Based on a regression of paleo temperatures onto the response to solar forcing simulated by an energy balance model, Scafetta and West (2007) find that up to $50 \%$ of the warming since 1900 may be solar-induced, but Benestad and Schmidt (2009) show this conclusion is not robust, being based on disregarding forcings other than solar in the preindustrial period, and assuming a high and precisely known value for climate sensitivity. Despite claims that more than half the warming since 1970 can be ascribed to solar variability (Loehle and Scaffetta, 2011) , a conclusion based on an incorrect assumption of no anthropogenic influence before 1950 and a 60-year solar cycle influence on global temperature (see also Mazzarella and Scafetta, 2012), several studies show that solar variations cannot explain global mean surface warming over the past 25 years, because solar irradiance has declined over this period (Lockwood and Fröhlich, 2007, 2008; Lockwood, 2008, 2012 ). Lean and Rind (2008) conclude that solar forcing explains only $10 \%$ of the warming over the past 100 years, while contributing a small cooling over the past 25 years. Thus while there is some evidence for solar influences on regional climate variability (Box 10.2) solar forcing has only had a small effect on GMST. Overall, we conclude that it is extremely unlikely that the contribution from solar forcing to the warming since 1950 was larger than that from GHGs.

A range of studies have used statistical methods to separate out the influence of known sources of internal variability, including ENSO and, in some cases, the AMO, from the response to external drivers, including volcanoes, solar variability and anthropogenic influence, in the recent GMST record: see, for example, Lockwood (2008), Lean and Rind (2009), Folland et al. (2013), Foster and Rahmstorf (2011) and Kaufmann et al. (2011). Representative results, as summarized in Imbers et al. (2013), are shown in Figure 10.6. These consistently attribute most of the warming over the past 50 years to anthropogenic influence, even allowing for potential confounding factors like the AMO. While results of such statistical approaches are sensitive to assumptions regarding the properties of both responses to external drivers and internal variability (Imbers et al., 2013), they provide a complementary approach to attribution studies based on global climate models.

Overall, given that the anthropogenic increase in GHGs likely caused $0.5^{\circ} \mathrm{C}$ to $1.3^{\circ} \mathrm{C}$ warming over $1951-2010$, with other anthropogenic forcings probably contributing counteracting cooling, that the effects of natural forcings and natural internal variability are estimated to be small, and that well-constrained and robust estimates of net anthropogenic warming are substantially more than half the observed warming (Figure 10.4) we conclude that it is extremely likely that human activities caused more than half of the observed increase in GMST from 1951 to 2010.

\section{The early 20th century warming}

The instrumental GMST record shows a pronounced warming during the first half of the 20th century (Figure 10.1a). Correction of residual biases in SST observations leads to a higher estimate of 1950s temperatures, but does not substantially change the warming between 1900 and 1940 (Morice et al., 2012). The AR4 concluded that 'the early 20th century warming is very likely in part due to external forcing' (Hegerl et al., 2007b), and that it is likely that anthropogenic forcing contributed to this warming. This assessment was based on studies including Shiogama et al. (2006) who find a contribution from solar and volcanic forcing to observed warming to 1949 , and Min and Hense (2006), who find strong evidence for a forced (either natural or combined natural and anthropogenic) contribution to global warming from 1900 to 1949. Ring et al. (2012) estimate, based on time series analysis, that part of the early 20th century warming was due to GHG increases (see also Figure 10.6), but find a dominant contribution by internal variability. CMIP5 model simulations of the historical period show forced warming over the early 20th century (Figure 10.1a), consistent with earlier detection and attribution analyses highlighted in the AR4 and TAR. The early 20th century contributes to the detection of external forcings over the 20th century estimated by detection and attribution results (Figure 10.4; Gillett et al., 2013; Ribes and Terray, 2013) and to the detected change over the last millennium to 1950 (see Figure 10.19; Schurer et al., 2013).

The pattern of warming and residual differences between models and observations indicate a role for circulation changes as a contributor to early 20th cenury warming (Figure 10.2), and the contribution of internal variability to the early 20th century warming has been analysed in several publications since the AR4. Crook and Forster (2011) find that the observed 1918-1940 warming was significantly greater than that simulated by most of the CMIP3 models. A distinguishing feature of the early 20th century warming is its pattern (Brönnimann, 2009) which shows the most pronounced warming in the Arctic during the cold season, followed by North America during the warm season, the North Atlantic Ocean and the tropics. In contrast, there was no unusual warming in Australia among other regions (see Figure 10.2b). Such a pronounced pattern points to a role for circulation change as a contributing factor to the regional anomalies contributing to this warming. Some studies have suggested that the warming is a response to the AMO (Schlesinger and Ramankutty, 1994; Polyakov et al., 2005; Knight et al., 2006; Tung and Zhou, 2013), or a large but random expression of internal variability (Bengtsson et al., 2006; Wood and Overland, 2010). Knight et al. (2009) diagnose a shift from the negative to the positive phase of the AMO from 1910 to 1940, a mode of circulation that is estimated to contribute approximately $0.1^{\circ} \mathrm{C}$, trough to peak, to GMST (Knight et al., 2005). Nonetheless, these studies do not challenge the AR4 assessment that external forcing very likely made a contribution to the warming over this period. In conclusion, the early 20th century warming is very unlikely to be due to internal variability alone. It remains difficult to quantify the contribution to this warming from internal variability, natural forcing and anthropogenic forcing, due to forcing and response uncertainties and incomplete observational coverage.

\section{Year-to-year and decade-to-decade variability of global mean surface temperature}

Time series analyses, such as those shown in Figure 10.6, seek to partition the variability of GMST into components attributable to anthropogenic and natural forcings and modes of internal variability such as ENSO and the AMO. Although such time series analyses support 
the major role of anthropogenic forcings, particularly due to increasing GHG concentrations, in contributing to the overall warming over the last 60 years, many factors, in addition to GHGs, including changes in tropospheric and stratospheric aerosols, stratospheric water vapour and solar output, as well as internal modes of variability, contribute to the year-to-year and decade-to-decade variability of GMST (Figure 10.6). Detailed discussion of the evolution of GMST of the past 15 years since 1998 is contained in Box 9.2.

\subsection{Attribution of regional surface temperature change}

Anthropogenic influence on climate has been robustly detected on the global scale, but for many applications an estimate of the anthropogenic contribution to recent temperature trends over a particular region is more useful. However, detection and attribution of climate change at continental and smaller scales is more difficult than on the global scale for several reasons (Hegerl et al., 2007b; Stott et al., 2010).

Estimated contributions to global mean temperature change
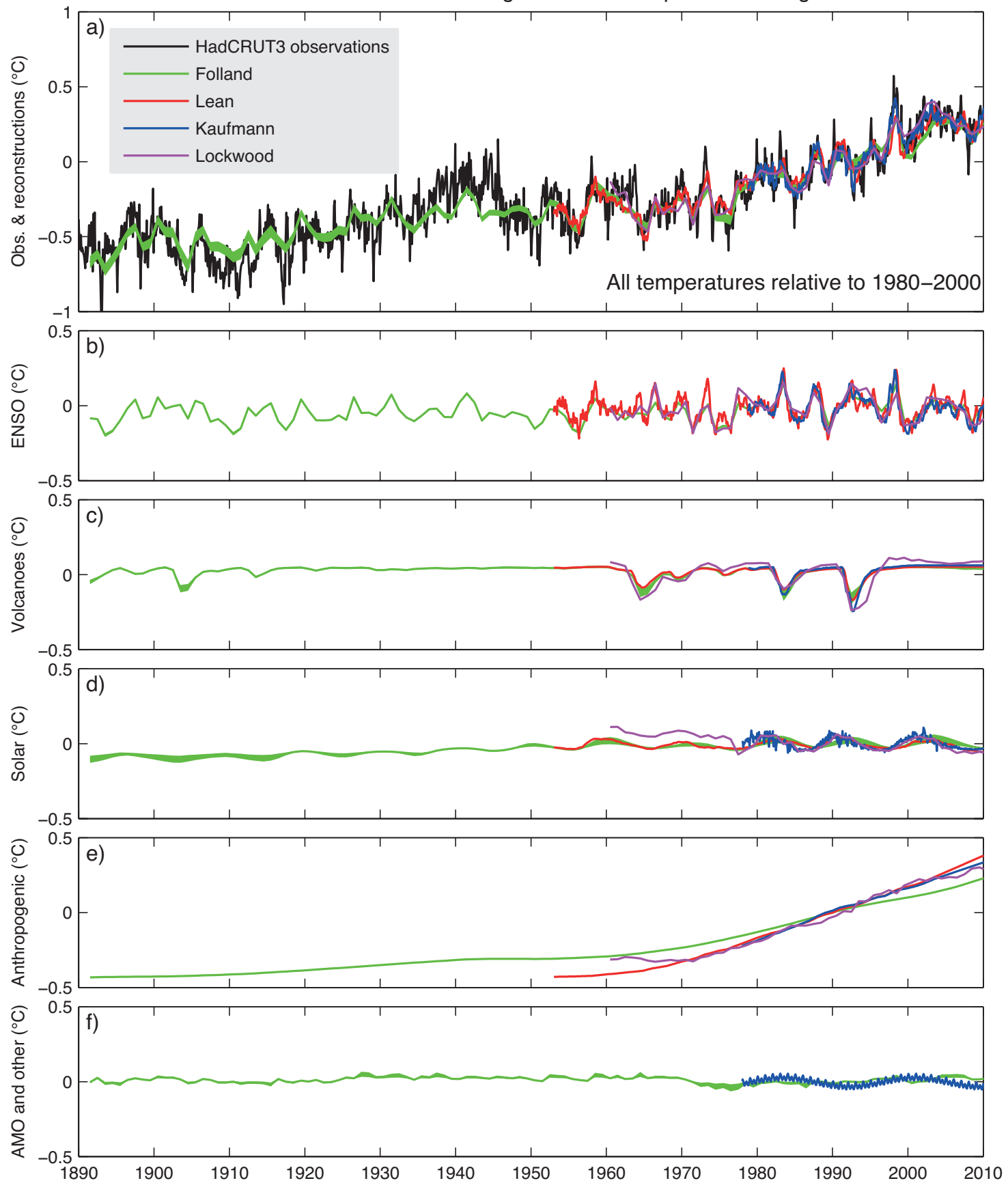

Figure 10.6 (Top) The variations of the observed global mean surface temperature (GMST) anomaly from Hadley Centre/Climatic Research Unit gridded surface temperature data set version 3 (HadCRUT3, black line) and the best multivariate fits using the method of Lean (red line), Lockwood (pink line), Folland (green line) and Kaufmann (blue line). (Below) The contributions to the fit from (a) El Niño-Southern Oscillation (ENSO), (b) volcanoes, (c) solar forcing, (d) anthropogenic forcing and (e) other factors (Atlantic Multi-decadal Oscillation (AMO) for Folland and a 17.5-year cycle, semi-annual oscillation (SAO), and Arctic Oscillation (AO) from Lean). (From Lockwood (2008), Lean and Rind (2009), Folland et al. (2013) and Kaufmann et al. (2011), as summarized in Imbers et al. (2013).) 


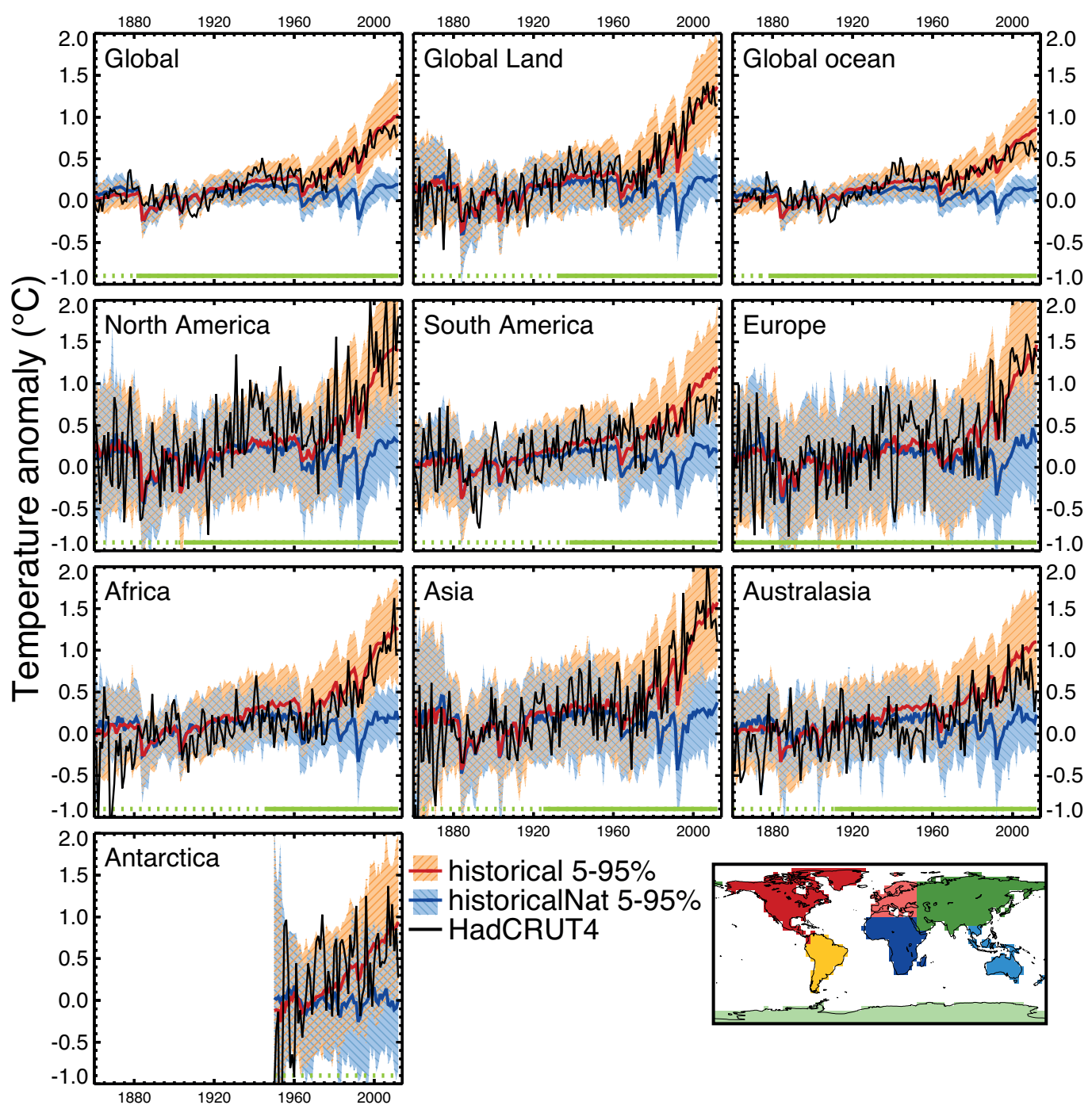

Figure 10.7 | Global, land, ocean and continental annual mean temperatures for CMIP3 and CMIP5 historical (red) and historicalNat (blue) simulations (multi-model means shown as thick lines, and 5 to 95\% ranges shown as thin light lines) and for Hadley Centre/Climatic Research Unit gridded surface temperature data set 4 (HadCRUT4, black). Mean temperatures are shown for Antarctica and six continental regions formed by combining the sub-continental scale regions defined by Seneviratne et al. (2012). Temperatures are shown with respect to 1880-1919 for all regions apart from Antarctica where temperatures are shown with respect to 1950-2010. (Adapted from Jones et al., 2013.)

First, the relative contribution of internal variability compared to the forced response to observed changes tends to be larger on smaller scales, as spatial differences in internal variations are averaged out in large-scale means. Second, because the patterns of response to climate forcings tend to be large scale, there is less spatial information to help distinguish between the responses to different forcings when attention is restricted to a sub-global area. Third, forcings omitted in some global climate model simulations may be important on regional scales, such as land use change or BC aerosol. Lastly, simulated internal variability and responses to forcings may be less reliable on smaller scales than on the global scale. Knutson et al. (2013) find a tendency for CMIP5 models to overestimate decadal variability in the $\mathrm{NH}$ extratropics in individual grid cells and underestimate it elsewhere, although Karoly and Wu (2005) and Wu and Karoly (2007) find that variability is not generally underestimated in earlier generation models.

Based on several studies, Hegerl et al. (2007b) concluded that 'it is likely that there has been a substantial anthropogenic contribution to surface temperature increases in every continent except Antarctica since the middle of the 20th century'. Figure 10.7 shows comparisons of observed continental scale temperatures (Morice et al., 2012) with CMIP5 simulations including both anthropogenic and natural forcings (red lines) and including just natural forcings (blue lines). Observed temperatures are largely within the range of simulations with anthropogenic forcings for all regions and outside the range of simulations with only natural forcings for all regions except Antarctica (Jones et al., 2013 ). Averaging over all observed locations, Antarctica has warmed over the 1950-2008 period (Section 2.4.1.1; Gillett et al., 2008b; Jones et al., 2013 ), even though some individual locations have cooled, particularly in summer and autumn, and over the shorter 1960-1999 period (Thompson and Solomon, 2002; Turner et al., 2005). When temperature changes associated with changes in the Southern Annular Mode are removed by regression, both observations and model simulations indicate warming at all observed locations except the South Pole over the 1950-1999 period (Gillett et al., 2008b). An analysis of Antarctic land temperatures over the period 1950-1999 
detected separate natural and anthropogenic responses of consistent magnitude in simulations and observations (Gillett et al., 2008b). Thus anthropogenic influence on climate has now been detected on all seven continents. However the evidence for human influence on Antarctic temperature is much weaker than for the other six continental regions. There is only one attribution study for this region, and there is greater observational uncertainty than the other regions, with very few data before 1950, and sparse coverage that is mainly limited to the coast and the Antarctic Peninsula. As a result of the observational uncertainties, there is low confidence in Antarctic region land surface air temperatures changes (Section 2.4.1.1) and we conclude for Antarctica there is low confidence that anthropogenic influence has contributed to the observed warming averaged over available stations.

Since the publication of the AR4 several other studies have applied attribution analyses to continental and sub-continental scale regions. Min and Hense (2007) applied a Bayesian decision analysis technique to continental-scale temperatures using the CMIP3 multi-model ensemble and concluded that forcing combinations including GHG increases provide the best explanation of 20th century observed changes in temperature on every inhabited continent except Europe, where the observational evidence is not decisive in their analysis. Jones et al. (2008) detected anthropogenic influence on summer temperatures over all $\mathrm{NH}$ continents and in many subcontinental $\mathrm{NH}$ land regions in an optimal detection analysis that considered the temperature responses to anthropogenic and natural forcings. Christidis et al. (2010) used a multi-model ensemble constrained by global-scale observed temperature changes to estimate the changes in probability of occurrence of warming or cooling trends over the 1950-1997 period over various sub-continental scale regions. They concluded that the probability of occurrence of warming trends had been at least doubled by anthropogenic forcing over all such regions except Central North America. The estimated distribution of warming trends over the Central North America region was approximately centred on the observed trend, so no inconsistency between simulated and observed trends was identified there. Knutson et al. (2013) demonstrated that observed temperature trends from the beginning of the observational record to 2010 averaged over Europe, Africa, Northern Asia, Southern Asia, Australia and South America are all inconsistent with the simulated response to natural forcings alone, and consistent with the simulated response to combined natural and anthropogenic forcings in the CMIP5 models. They reached a similar conclusion for the major ocean basins with the exception of the North Atlantic, where variability is high.

Several recent studies have applied attribution analyses to specific sub-continental regions. Anthropogenic influence has been found in winter minimum temperature over the western USA (Bonfils et al., 2008; Pierce et al., 2009), a conclusion that is found to be robust to weighting models according to various aspects of their climatology (Pierce et al., 2009); anthropogenic influence has been found in temperature trends over New Zealand (Dean and Stott, 2009) after circulation-related variability is removed as in Gillett et al. (2000); and anthropogenic influence has been found in temperature trends over France, using a first-order autoregressive model of internal variability (Ribes et al., 2010). Increases in anthropogenic GHGs were found to be the main driver of the 20th-century SST increases in both Atlantic and Pacific tropical cyclogenesis regions (Santer et al., 2006; Gillett et al., 2008a). Over both regions, the response to anthropogenic forcings is detected when the response to natural forcings is also included in the analysis (Gillett et al., 2008a). Knutson et al. (2013) detect an anthropogenic influence over Canada, but not over the continental USA, Alaska or Mexico.

Gillett et al. (2008b) detect anthropogenic influence on near-surface Arctic temperatures over land, with a consistent magnitude in simulations and observations. Wang et al. (2007) also find that observed Arctic warming is inconsistent with simulated internal variability. Both studies ascribe Arctic warmth in the 1930s and 1940s largely to internal variability. Shindell and Faluvegi (2009) infer a large contribution to both mid-century Arctic cooling and late century warming from aerosol forcing changes, with GHGs the dominant driver of long-term warming, though they infer aerosol forcing changes from temperature changes using an inverse approach which may lead to some changes associated with internal variability being attributed to aerosol forcing. We therefore conclude that despite the uncertainties introduced by limited observational coverage, high internal variability, modelling uncertainties (Crook et al., 2011) and poorly understood local forcings, such as the effect of BC on snow, there is sufficiently strong evidence to conclude that it is likely that there has been an anthropogenic contribution to the very substantial warming in Arctic land surface temperatures over the past 50 years.

Some attribution analyses have considered temperature trends at the climate model grid box scale. At these spatial scales robust attribution is difficult to obtain, since climate models often lack the processes needed to simulate regional details realistically, regionally important forcings may be missing in some models and observational uncertainties are very large for some regions of the world at grid box scale (Hegerl et al., 2007b; Stott et al., 2010). Nevertheless an attribution analysis has been carried out on Central England temperature, a record that extends back to 1659 and is sufficiently long to demonstrate that the representation of multi-decadal variability in the single grid box in the model used, Hadley Centre climate prediction model 3 (HadCM3) is adequate for detection (Karoly and Stott, 2006). The observed trend in Central England Temperature is inconsistent with either internal variability or the simulated response to natural forcings, but is consistent with the simulated response when anthropogenic forcings are included (Karoly and Stott, 2006).

Observed 20th century grid cell trends from Hadley Centre/Climatic Research Unit gridded surface temperature data set 2v (HadCRUT2v; Jones et al., 2001) are inconsistent with simulated internal variability at the $10 \%$ significance level in around $80 \%$ of grid cells even using HadCM2 which was found to overestimate variability in 5 -year mean temperatures at most latitudes (Karoly and Wu, 2005). Sixty percent of grid cells were found to exhibit significant warming trends since 1951, a much larger number than expected by chance (Karoly and Wu, 2005; Wu and Karoly, 2007), and similar results apply when circulation-related variability is first regressed out (Wu and Karoly, 2007). However, as discussed in the AR4 (Hegerl et al., 2007b), when a global field significance test is applied, this becomes a global detection study; since not all grid cells exhibit significant warming trends the overall interpretation of the results in terms of attribution at individual locations remains problematic. Mahlstein et al. (2012) find significant changes in summer season temperatures in about $40 \%$ of low-latitude and about $20 \%$ of 
extratropical land grid cells with sufficient observations, when testing against the null hypothesis of no change in the distribution of summer temperatures. Observed grid cell trends are compared with CMIP5 simulated trends in Figure 10.2i, which shows that in the great majority $(89 \%)$ of grid cells with sufficient observational coverage, observed trends over the 1901-2010 period are inconsistent with a combination of simulated internal variability and the response to natural forcings (Jones et al., 2013). Knutson et al. (2013) find some deficiencies in the simulation of multi-decadal variability at the grid cell scale in CMIP5 models, but demonstrate that trends at more than $75 \%$ of individual grid cells with sufficient observational coverage in HadCRUT4 are inconsistent with the simulated response to natural forcings alone, and consistent or larger than the simulated response to combined anthropogenic and natural forcings in CMIP5 models.

In summary, it is likely that anthropogenic forcing has made a substantial contribution to the warming of each of the inhabited continents since 1950. For Antarctica large observational uncertainties result in low confidence that anthropogenic influence has contributed to the observed warming averaged over available stations. Anthropogenic influence has likely contributed to temperature change in many sub-continental regions. Detection and attribution of climate change at continental and smaller scales is more difficult than at the global scale due to the greater contribution of internal variability, the greater difficulty of distinguishing between different causal factors, and greater errors in climate models' representation of regional details. Nevertheless, statistically significant warming trends are observed at a majority of grid cells, and the observed warming is inconsistent with estimates of possible warming due to natural causes at the great majority of grid cells with sufficient observational coverage.

\subsubsection{Atmosphere}

This section presents an assessment of the causes of global and regional temperature changes in the free atmosphere. In AR4, Hegerl et al. (2007b) concluded that 'the observed pattern of tropospheric warming and stratospheric cooling is very likely due to the influence of anthropogenic forcing, particularly greenhouse gases and stratospheric ozone depletion.' Since AR4, insight has been gained into regional aspects of free tropospheric trends and the causes of observed changes in stratospheric temperature.

Atmospheric temperature trends through the depth of the atmosphere offer the possibility of separating the effects of multiple climate forcings, as climate model simulations indicate that each external forcing produces a different characteristic vertical and zonal pattern of temperature response (Hansen et al., 2005b; Hegerl et al., 2007b; Penner et al., 2007; Yoshimori and Broccoli, 2008). GHG forcing is expected to warm the troposphere and cool the stratosphere. Stratospheric ozone depletion cools the stratosphere, with the cooling being most pronounced in the polar regions. Its effect on tropospheric temperatures is small, which is consistent with a small estimated RF of stratospheric ozone changes (SPARC CCMVal, 2010; McLandress et al., 2012). Tropospheric ozone increase, on the other hand, causes tropospheric warming. Reflective aerosols like sulphate cool the troposphere while absorbing aerosols like $\mathrm{BC}$ have a warming effect. Free atmosphere temperatures are also affected by natural forcings: solar irradiance increases cause a general warming of the atmosphere and volcanic aerosol ejected into the stratosphere causes tropospheric cooling and stratospheric warming (Hegerl et al., 2007b).

\subsection{Tropospheric temperature change}

Chapter 2 concludes that it is virtually certain that globally the troposphere has warmed since the mid-twentieth century with only medium (NH extratropics) to low confidence (tropics and SH extratropics) in the rate and vertical structure of these changes. During the satellite era CMIP3 and CMIP5 models tend to warm faster than observations specifically in the tropics (McKitrick et al., 2010; Fu et al., 2011; Po-Chedley and Fu, 2012; Santer et al., 2013); however, because of the large uncertainties in observed tropical temperature trends (Section 2.4.4; Seidel et al. (2012); Figures 2.26 and Figure 2.27) there is only low confidence in this assessment (Section 9.4.1.4.2). Outside the tropics, and over the period of the radiosonde record beginning in 1961, the discrepancy between simulated and observed trends is smaller (Thorne et al., 2011; Lott et al., 2013; Santer et al., 2013). Specifically there is better agreement between observed trends and CMIP5 model trends for the NH extratropics (Lott et al., 2013). Factors other than observational uncertainties that contribute to inconsistencies between observed and simulated free troposphere warming include specific manifestation of natural variability in the observed coupled atmosphere-ocean system, forcing errors incorporated in the historical simulations and model response errors (Santer et al., 2013).

Utilizing a subset of CMIP5 models with single forcing experiments extending until 2010, Lott et al. (2013) detect influences of both human induced GHG increase and other anthropogenic forcings (e.g., ozone and aerosols) in the spatio-temporal changes in tropospheric temperatures from 1961 to 2010 estimated from radiosonde observations. Figure 10.8 illustrates that a subsample of CMIP5 models (see Supplementary Material for model selection) forced with both anthropogenic and natural climate drivers (red profiles) exhibit trends that are consistent with radiosonde records in the troposphere up to about $300 \mathrm{hPa}$, albeit with a tendency for this subset of models to warm more than the observations. This finding is seen in near-globally averaged data (where there is sufficient observational coverage to make a meaningful comparison: $60^{\circ} \mathrm{S}$ to $60^{\circ} \mathrm{N}$ ) (right panel), as well as in latitudinal bands of the SH extratropics (Figure 10.8, first panel), tropics (Figure 10.8, second panel) and the NH extratropics (Figure 10.8, third panel). Figure 10.8 also illustrates that it is very unlikely that natural forcings alone could have caused the observed warming of tropospheric temperatures (blue profiles). The ensembles with both anthropogenic and natural forcings (red) and with GHG forcings only (green) are not clearly separated. This could be due to cancellation of the effects of increases in reflecting aerosols, which cool the troposphere, and absorbing aerosol (Penner et al., 2007) and tropospheric ozone, which both warm the troposphere. Above $300 \mathrm{hPa}$ the three radiosonde data sets exhibit a larger spread as a result of larger uncertainties in the observational record (Thorne et al., 2011; Section 2.4.4). In this region of the upper troposphere simulated CMIP5 temperature trends tend to be more positive than observed trends (Figure 10.8). Further, an assessment of causes of observed trends in the upper troposphere is less confident than an assessment of overall atmospheric temperature changes because of observational uncertainties and potential remain- 

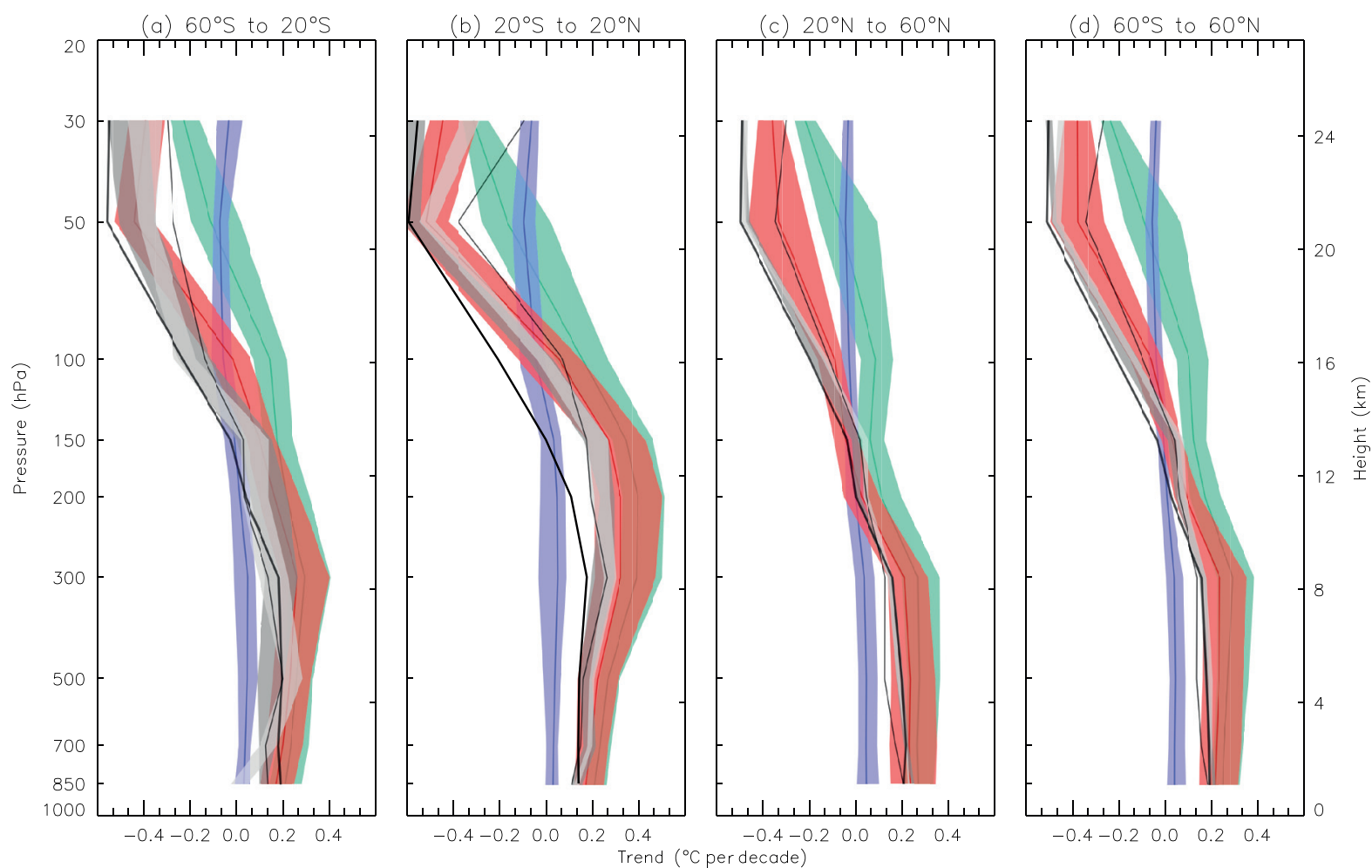

Figure 10.8 Observed and simulated zonal mean temperatures trends from 1961 to 2010 for CMIP5 simulations containing both anthropogenic and natural forcings (red), natural forcings only (blue) and greenhouse gas forcing only (green) where the 5 to 95 th percentile ranges of the ensembles are shown. Three radiosonde observations are shown (thick black line: Hadley Centre Atmospheric Temperature data set 2 (HadAT2), thin black line: RAdiosone OBservation COrrection using REanalyses 1.5 (RAOBCORE 1.5), dark grey band: Radiosonde Innovation Composite Homogenization (RICH)-obs 1.5 ensemble and light grey: RICH- $\tau$ 1.5 ensemble. (After Lott et al., 2013.)

ing systematic biases in observational data sets in this region (Thorne et al., 2011; Haimberger et al., 2012). An analysis of contributions of natural and anthropogenic forcings to more recent trends from 1979 to 2010 (Supplementary Material, Figure S.A.1) is less robust because of increased uncertainty in observed trends (consistent with Seidel et al. (2012)) as well as decreased capability to separate between individual forcings ensembles.

One approach to identify a climate change signal in a time series is the analysis of the ratio between the amplitude of the observed signal of change divided by the magnitude of internal variability, in other words the $S / N$ ratio of the data record. The $S / N$ ratio represents the result of a non-optimal fingerprint analysis (in contrast to optimal fingerprint analyses where model-simulated responses and observations are normalized by internal variability to improve the $\mathrm{S} / \mathrm{N}$ ratio (see Section 10.2.3). For changes in the lower stratospheric temperature between 1979 and 2011, S/N ratios vary from 26 to 36, depending on the choice of observational data set. In the lower troposphere, the fingerprint strength in observations is smaller, but $\mathrm{S} / \mathrm{N}$ ratios are still significant at the $1 \%$ level or better, and range from 3 to 8 . There is no evidence that these ratios are spuriously inflated by model variability errors. After all global mean signals are removed, model fingerprints remain identifiable in $70 \%$ of the tests involving tropospheric temperature changes (Santer et al., 2013).

Hegerl et al. (2007a) concluded that increasing GHGs are the main cause for warming of the troposphere. This result is supported by a subsample of CMIP5 models that also suggest that the warming effect of well mixed GHGs is partly offset by the combined effects of reflecting aerosols and other forcings. Our understanding has been increased regarding the time scale of detectability of global scale troposphere temperature. Taken together with increased understanding of the uncertainties in observational records of tropospheric temperatures (including residual systematic biases; Section 2.4.4) the assessment remains as it was for AR4 that it is likely that anthropogenic forcing has led to a detectable warming of tropospheric temperatures since 1961.

\subsection{Stratospheric temperature change}

Lower stratospheric temperatures have not evolved uniformly over the period since 1958 when the stratosphere has been observed with sufficient regularity and spatial coverage. A long-term global cooling trend is interrupted by three 2-year warming episodes following large volcanic eruptions (Section 2.4.4). During the satellite period the cooling evolved mainly in two steps occurring in the aftermath of the El Chichón eruption in 1982 and the Mt Pinatubo eruption of 1991, with each cooling transition being followed by a period of relatively steady temperatures (Randel et al., 2009; Seidel et al., 2011). Since the mid1990s little net change has occurred in lower stratospheric temperatures (Section 2.4.4).

Since AR4, progress has been made in simulating the observed evolution of global mean lower stratospheric temperature. On the one hand, this has been achieved by using models with an improved 
representation of stratospheric processes (chemistry-climate models and some CMIP5 models). It is found that in these models which have an upper boundary above the stratopause with an altitude of about $50 \mathrm{~km}$ (so-called high-top models) and improved stratospheric physics, variability of lower stratosphere climate in general is well simulated (Butchart et al., 2011; Gillett et al., 2011; Charlton-Perez et al., 2013) whereas in so-called low-top models (including models participating in CMIP3) it is generally underestimated (Cordero and Forster, 2006; Charlton-Perez et al., 2013). On the other hand, CMIP5 models all include changes in stratospheric ozone (Eyring et al., 2013) whereas only about half of the models participating in CMIP3 include stratospheric ozone changes (Section 9.4.1.4.5). A comparison of a low-top and high-top version of the HadGEM2 model shows detectable differences in modelled temperature changes, particularly in the lower tropical stratosphere, with the high-top version's simulation of temperature trends in the tropical troposphere in better agreement with radiosondes and reanalyses over 1981-2010 (Mitchell et al., 2013).

CMIP5 models forced with changes in WMGHGs and stratospheric ozone as well as with changes in solar irradiance and volcanic aerosol forcings simulate the evolution of observed global mean lower stratospheric temperatures over the satellite era reasonably well although they tend to underestimate the long-term cooling trend (Charlton-Perez et al., 2013; Santer et al., 2013). Compared with radiosonde data the cooling trend is also underestimated in a subset of CMIP5 simulations over the period 1961-2010 (Figure 10.8) and in CMIP3 models over the 1958-1999 period (Cordero and Forster, 2006). Potential causes for biases in lower stratosphere temperature trends are observational uncertainties (Section 2.4.4) and forcing errors related to prescribed stratospheric aerosol loadings and stratospheric ozone changes affecting the tropical lower stratosphere (Free and Lanzante, 2009; Solomon et al., 2012; Santer et al., 2013).

Since AR4, attribution studies have improved our knowledge of the role of anthropogenic and natural forcings in observed lower stratospheric temperature change. Gillett et al. (2011) use the suite of chemistry climate model simulations carried out as part of the Chemistry Climate Model Validation (CCMVal) activity phase 2 for an attribution study of observed changes in stratospheric zonal mean temperatures. Chemistry-climate models prescribe changes in ozone-depleting substances (ODS) and ozone changes are calculated interactively. Gillett et al. (2011) partition 1979-2005 Microwave Sounding Unit (MSU) lower stratospheric temperature trends into ODS-induced and GHG-induced changes and find that both ODSs and natural forcing contributed to the observed stratospheric cooling in the lower stratosphere with the impact of ODS dominating. The influence of GHGs on stratospheric temperature could not be detected independently of ODSs.

The step-like cooling of the lower stratosphere can only be explained by the combined effects of changes in both anthropogenic and natural factors (Figure 10.9; Eyring et al., 2006; Ramaswamy et al., 2006). Although the anthropogenic factors (ozone depletion and increases in WMGHGs) cause the overall cooling, the natural factors (solar irradiance variations and volcanic aerosols) modulate the evolution of the cooling (Figure 10.9; Ramaswamy et al., 2006; Dall'Amico et al., 2010) with temporal variability of global mean ozone contributing to the step-like temperature evolution (Thompson and Solomon, 2009).

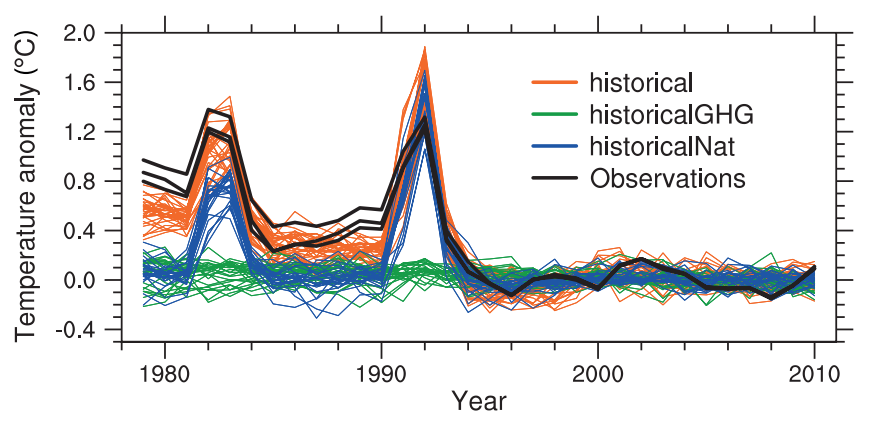

Figure 10.9 | Time series (1979-2010) of observed (black) and simulated global mean $\left(82.5^{\circ} \mathrm{S}\right.$ to $82.5^{\circ} \mathrm{N}$ ) Microwave Sounding Unit (MSU) lower stratosphere temperature anomalies in a subset of CMIP5 simulations (simulations with both anthropogenic and natural forcings (red), simulations with well-mixed greenhouse gases (green), simulations with natural forcings (blue)). Anomalies are calculated relative to 1996-2010. (Adapted from Ramaswamy et al., 2006.)

Models disagree with observations for seasonally varying changes in the strength of the Brewer-Dobson circulation in the lower stratosphere (Ray et al., 2010) which has been linked to zonal and seasonal patterns of changes in lower stratospheric temperatures (Thompson and Solomon, 2009; Fu et al., 2010; Lin et al., 2010b; Forster et al., 2011; Free, 2011). One robust feature is the observed cooling in spring over the Antarctic, which is simulated in response to stratospheric ozone depletion in climate models (Young et al., 2012), although this has not been the subject of a formal detection and attribution study.

Since AR4, progress has been made in simulating the response of global mean lower stratosphere temperatures to natural and anthropogenic forcings by improving the representation of climate forcings and utilizing models that include more stratospheric processes. New detection and attribution studies of lower stratospheric temperature changes made since AR4 support an assessment that it is very likely that anthropogenic forcing, dominated by stratospheric ozone depletion due to ozone-depleting substances, has led to a detectable cooling of the lower stratosphere since 1979.

\subsection{Overall atmospheric temperature change}

When temperature trends from the troposphere and stratosphere are analysed together, detection and attribution studies using CMIP5 models show robust detections of the effects of GHGs and other anthropogenic forcings on the distinctive fingerprint of tropospheric warming and stratospheric cooling seen since 1961 in radiosonde data (Lott et al., 2013; Mitchell et al., 2013). Combining the evidence from free atmosphere changes from both troposphere and stratosphere shows an increased confidence in the attribution of free atmosphere temperature changes compared to AR4 owing to improved understanding of stratospheric temperature changes. There is therefore stronger evidence than at the time of AR4 to support the conclusion that it is very likely that anthropogenic forcing, particularly GHGs and stratospheric ozone depletion, has led to a detectable observed pattern of tropospheric warming and lower stratospheric cooling since 1961. 
Frequently Asked Questions

\section{FAQ 10.1 | Climate Is Always Changing. How Do We Determine the Causes of Observed Changes?}

The causes of observed long-term changes in climate (on time scales longer than a decade) are assessed by determining whether the expected 'fingerprints' of different causes of climate change are present in the historical record. These fingerprints are derived from computer model simulations of the different patterns of climate change caused by individual climate forcings. On multi-decade time scales, these forcings include processes such as greenhouse gas increases or changes in solar brightness. By comparing the simulated fingerprint patterns with observed climate changes, we can determine whether observed changes are best explained by those fingerprint patterns, or by natural variability, which occurs without any forcing.

The fingerprint of human-caused greenhouse gas increases is clearly apparent in the pattern of observed 20th century climate change. The observed change cannot be otherwise explained by the fingerprints of natural forcings or natural variability simulated by climate models. Attribution studies therefore support the conclusion that 'it is extremely likely that human activities have caused more than half of the observed increase in global mean surface temperatures from 1951 to 2010.'

The Earth's climate is always changing, and that can occur for many reasons. To determine the principal causes of observed changes, we must first ascertain whether an observed change in climate is different from other fluctuations that occur without any forcing at all. Climate variability without forcing — called internal variability-is the consequence of processes within the climate system. Large-scale oceanic variability, such as El Niño-Southern Oscillation (ENSO) fluctuations in the Pacific Ocean, is the dominant source of internal climate variability on decadal to centennial time scales.

Climate change can also result from natural forcings external to the climate system, such as volcanic eruptions, or changes in the brightness of the sun. Forcings such as these are responsible for the huge changes in climate that are clearly documented in the geological record. Human-caused forcings include greenhouse gas emissions or atmospheric particulate pollution. Any of these forcings, natural or human caused, could affect internal variability as well as causing a change in average climate. Attribution studies attempt to determine the causes of a detected change in observed climate. Over the past century we know that global average temperature has increased, so if the observed change is forced then the principal forcing must be one that causes warming, not cooling.

Formal climate change attribution studies are carried out using controlled experiments with climate models. The model-simulated responses to specific climate forcings are often called the fingerprints of those forcings. A climate model must reliably simulate the fingerprint patterns associated with individual forcings, as well as the patterns of unforced internal variability, in order to yield a meaningful climate change attribution assessment. No model can perfectly reproduce all features of climate, but many detailed studies indicate that simulations using current models are indeed sufficiently reliable to carry out attribution assessments.

FAQ 10.1, Figure 1 illustrates part of a fingerprint assessment of global temperature change at the surface during the late 20th century. The observed change in the latter half of the 20th century, shown by the black time series in the left panels, is larger than expected from just internal variability. Simulations driven only by natural forcings (yellow and blue lines in the upper left panel) fail to reproduce late 20th century global warming at the surface with a spatial pattern of change (upper right) completely different from the observed pattern of change (middle right). Simulations including both natural and human-caused forcings provide a much better representation of the time rate of change (lower left) and spatial pattern (lower right) of observed surface temperature change.

Both panels on the left show that computer models reproduce the naturally forced surface cooling observed for a year or two after major volcanic eruptions, such as occurred in 1982 and 1991. Natural forcing simulations capture the short-lived temperature changes following eruptions, but only the natural + human caused forcing simulations simulate the longer-lived warming trend.

A more complete attribution assessment would examine temperature above the surface, and possibly other climate variables, in addition to the surface temperature results shown in FAQ 10.1, Figure 1. The fingerprint patterns associated with individual forcings become easier to distinguish when more variables are considered in the assessment. 
Overall, FAQ 10.1, Figure 1 shows that the pattern of observed temperature change is significantly different than the pattern of response to natural forcings alone. The simulated response to all forcings, including human-caused forcings, provides a good match to the observed changes at the surface. We cannot correctly simulate recent observed climate change without including the response to human-caused forcings, including greenhouse gases, stratospheric ozone, and aerosols. Natural causes of change are still at work in the climate system, but recent trends in temperature are largely attributable to human-caused forcing.
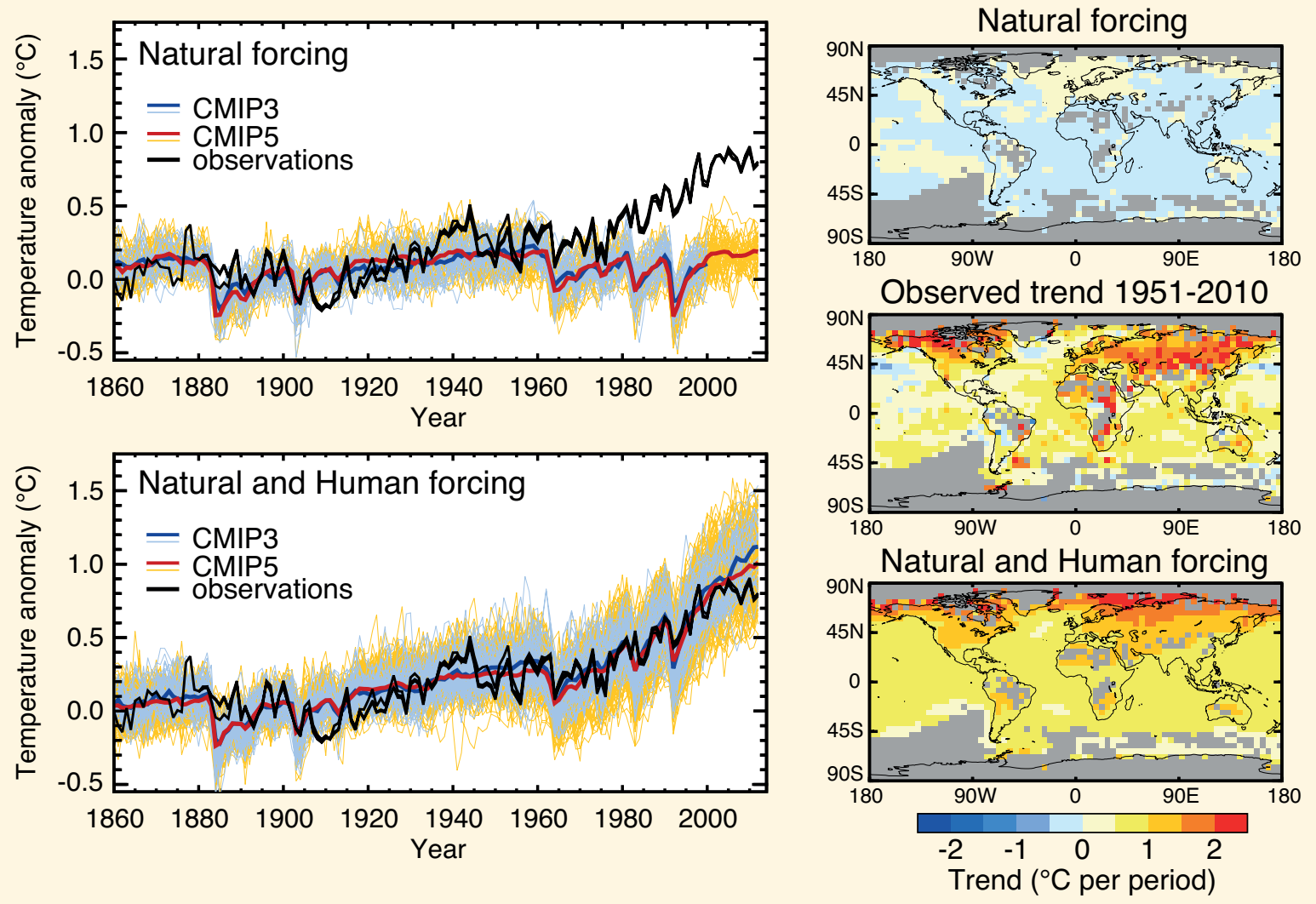

FAQ 10.1, Figure 1| (Left) Time series of global and annual-averaged surface temperature change from 1860 to 2010. The top left panel shows results from two ensemble of climate models driven with just natural forcings, shown as thin blue and yellow lines; ensemble average temperature changes are thick blue and red lines. Three different observed estimates are shown as black lines. The lower left panel shows simulations by the same models, but driven with both natural forcing and human-induced changes in greenhouse gases and aerosols. (Right) Spatial patterns of local surface temperature trends from 1951 to 2010. The upper panel shows the pattern of trends from a large ensemble of Coupled Model Intercomparison Project Phase 5 (CMIP5) simulations driven with just natural forcings. The bottom panel shows trends from a corresponding ensemble of simulations driven with natural + human forcings. The middle panel shows the pattern of observed trends from the Hadley Centre/Climatic Research Unit gridded surface temperature data set 4 (HadCRUT4) during this period.

\subsubsection{Water Cycle}

Detection and attribution studies of anthropogenic change in hydrologic variables are challenged by the length and quality of observed data sets, and by the difficulty in simulating hydrologic variables in dynamical models. AR4 cautiously noted that the observed increase in atmospheric water vapour over oceans was consistent with warming of SSTs attributed to anthropogenic influence, and that observed changes in the latitudinal distribution of precipitation, and increased incidence of drought, were suggestive of a possible human influence. Many of the published studies cited in AR4, and some of the studies cited in this section, use less formal detection and attribution criteria than are often used for assessments of temperature change, owing to difficulties defining large-scale fingerprint patterns of hydrologic change in models and isolating those fingerprints in data. For example, correlations between observed hydrologic changes and the patterns of change in models forced by increasing GHGs can provide suggestive evidence towards attribution of change.

Since the publication of AR4, in situ hydrologic data sets have been reanalysed with more stringent quality control. Satellite-derived data records of worldwide water vapour and precipitation variations have 
lengthened. Formal detection and attribution studies have been carried out with newer models that potentially offer better simulations of natural variability. Reviews of detection and attribution of trends in various components of the water cycle have been published by Stott et al. (2010) and Trenberth (2011b).

\subsubsection{Changes in Atmospheric Water Vapour}

In situ surface humidity measurements have been reprocessed since AR4 to create new gridded analyses for climatic research, as discussed in Chapter 2. The HadCRUH Surface Humidity data set (Willett et al., 2008) indicates significant increases in surface specific humidity between 1973 and 2003 averaged over the globe, the tropics, and the $\mathrm{NH}$, with consistently larger trends in the tropics and in the $\mathrm{NH}$ during summer, and negative or non significant trends in relative humidity. These results are consistent with the hypothesis that the distribution of relative humidity should remain roughly constant under climate change (see Section 2.5). Simulations of the response to historical anthropogenic and natural forcings robustly generate an increase in atmospheric humidity consistent with observations (Santer et al., 2007; Willett et al., 2007; Figure 9.9). A recent cessation of the upward trend in specific humidity is observed over multiple continental areas in HadCRUH and is also found in the European Centre for Medium range Weather Forecast (ECMWF) interim reanalysis of the global atmosphere and surface conditions (ERA-Interim; Simmons et al. 2010). This change in the specific humidity trend is temporally correlated with a levelling off of global ocean temperatures following the 1997-1998 El Niño event (Simmons et al., 2010).

The anthropogenic water vapour fingerprint simulated by an ensemble of 22 climate models has been identified in lower tropospheric moisture content estimates derived from Special Sensor Microwave/lmager (SSM/I) data covering the period 1988-2006 (Santer et al., 2007). Santer et al. (2009) find that detection of an anthropogenic response in column water vapour is insensitive to the set of models used. They rank models based on their ability to simulate the observed mean total column water vapour, and its annual cycle and variability associated with ENSO. They report no appreciable differences between the fingerprints or detection results derived from the best or worst performing models, and so conclude that attribution of water vapour changes to anthropogenic forcing is not sensitive to the choice of models used for the assessment.

In summary, an anthropogenic contribution to increases in specific humidity at and near the Earth's surface is found with medium confidence. Evidence of a recent levelling off of the long-term surface atmospheric moistening trend over land needs to be better understood and simulated as a prerequisite to increased confidence in attribution studies of water vapour changes. Length and quality of observational humidity data sets, especially above the surface, continue to limit detection and attribution studies of atmospheric water vapour.

\subsubsection{Changes in Precipitation}

Analysis of CMIP5 model simulations yields clear global and regional scale changes associated with anthropogenic forcing (e.g., Scheff and Frierson, 2012a, 2012b), with patterns broadly similar to those identified from CMIP3 models (e.g., Polson et al., 2013). The AR4 concluded that 'the latitudinal pattern of change in land precipitation and observed increases in heavy precipitation over the 20th century appear to be consistent with the anticipated response to anthropogenic forcing'. Detection and attribution of regional precipitation changes generally focuses on continental areas using in situ data because observational coverage over oceans is limited to a few island stations (Arkin et al., 2010; Liu et al., 2012; Noake et al., 2012) , although model-data comparisons over continents also illustrate large observational uncertainties (Tapiador, 2010; Noake et al., 2012; Balan Sarojini et al., 2012; Polson et al., 2013). Available satellite data sets that could supplement oceanic studies are short and their long-term homogeneity is still unclear (Chapter 2); hence they have not yet been used for detection and attribution of changes. Continuing uncertainties in climate model simulations of precipitation make quantitative model/data comparisons difficult (e.g., Stephens et al., 2010), which also limits confidence in detection and attribution. Furthermore, sparse observational coverage of precipitation across much of the planet makes the fingerprint of precipitation change challenging to isolate in observational records (Balan Sarojini et al., 2012; Wan et al., 2013).

Considering just land regions with sufficient observations, the largest signal of differences between models with and without anthropogenic forcings is in the high latitudes of the $\mathrm{NH}$, where increases in precipitation are a robust feature of climate model simulations (Scheff and Frierson, 2012a, 2012b). Such increases have been observed (Figure 10.10) in several different observational data sets (Min et al., 2008a; Noake et al., 2012; Polson et al., 2013), although high-latitude trends vary between data sets and with coverage (e.g., Polson et al., 2013).

Attribution of zonally averaged precipitation trends has been attempted using different observational products and ensembles of forced simulations from both the CMIP3 and CMIP5 archives, for annual-averaged (Zhang et al., 2007; Min et al., 2008a) and season-specific (Noake et al., 2012; Polson et al., 2013) results (Figure 10.11). Zhang et al. (2007) identify the fingerprint of anthropogenic changes in observed annual zonal mean precipitation averaged over the periods 1925-1999 and 1950-1999, and separate the anthropogenic fingerprint from the influence of natural forcing. The fingerprint of external forcing is also detected in seasonal means for boreal spring in all data sets assessed by Noake et al. (2012), and in all but one data set assessed by Polson et al. (2013) (Figure 10.11), and in boreal winter in all but one data set (Noake et al., 2012), over the period 1951-1999 and to 2005. The fingerprint features increasing high-latitude precipitation, and decreasing precipitation trends in parts of the tropics that are reasonably robustly observed in all four data sets considered albeit with large observational uncertainties north of $60^{\circ} \mathrm{N}$ (Figure 10.11). Detection of seasonal-average precipitation change is less convincing for June, July, August (JJA) and September, October, November (SON) and results vary with observation data set (Noake et al., 2012; Polson et al., 2013). Although Zhang et al. (2007) detect anthropogenic changes even if a separate fingerprint for natural forcings is considered, Polson et al. (2013) find that this result is sensitive to the data set used and that the fingerprints can be separated robustly only for the data set most closely constrained by station data. The analysis also finds that model simulated precipitation variability is smaller than observed variability in the tropics (Zhang et al., 2007; 
Polson et al., 2013) which is addressed by increasing the estimate of variance from models (Figure 10.11).

Another detection and attribution study focussed on precipitation in the NH high latitudes and found an attributable human influence (Min et al., 2008a). Both Min et al. (2008a) and Zhang et al. (2007) find that the observed changes are significantly larger than the model simulated changes. However, Noake et al. (2012) and Polson et al. (2013) find that the difference between models and observations decreases if changes

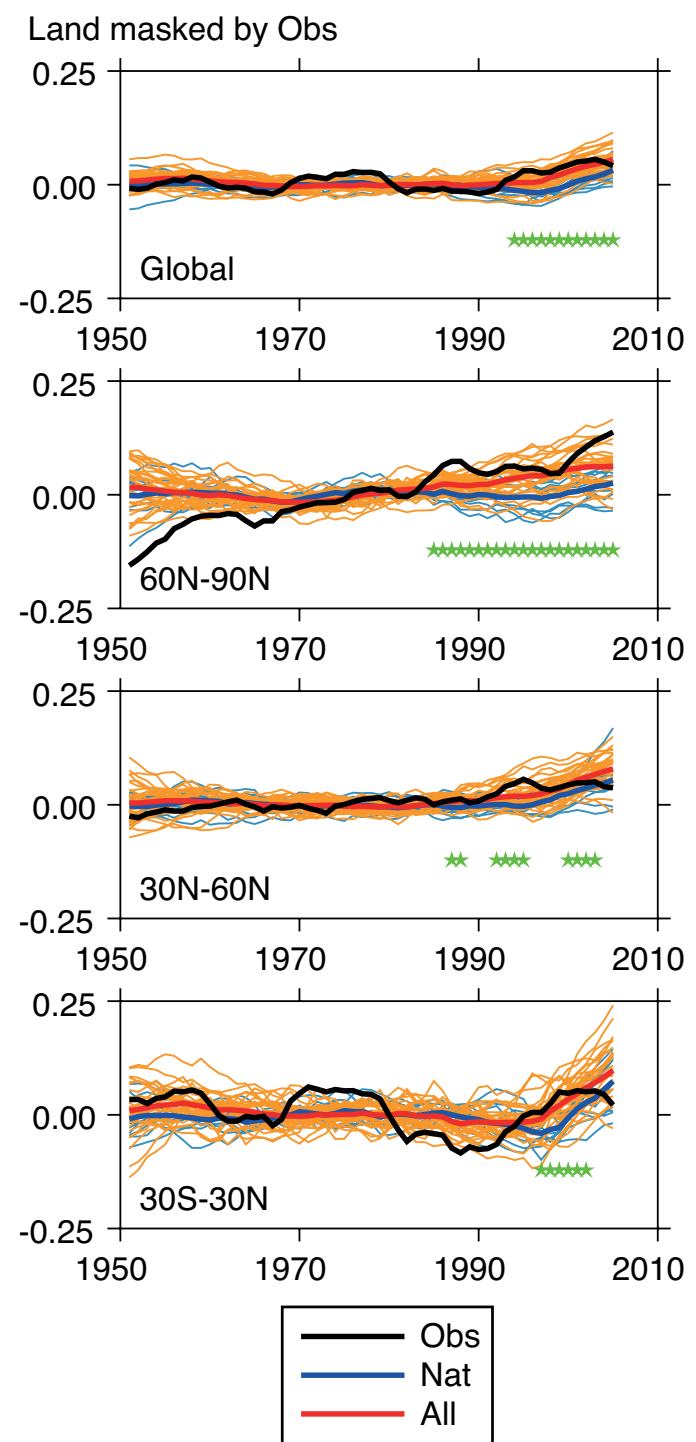

Figure 10.10 | Global and zonal average changes in annual mean precipitation (mm day $^{-1}$ ) over areas of land where there are observations, expressed relative to the baseline period of 1961-1990, simulated by CMIP5 models forced with both anthropogenic and natural forcings (red lines) and natural forcings only (blue lines) for the global mean and for three latitude bands. Multi-model means are shown in thick solid lines. Observations (gridded values derived from Global Historical Climatology Network station data, updated from Zhang et al. (2007) are shown as a black solid line. An 11-year smoothing is applied to both simulations and observations. Green stars show statistically significant changes at $5 \%$ level ( $p$ value $<0.05$ ) between the ensemble of runs with both anthropogenic and natural forcings (red lines) and the ensemble of runs with just natural forcings (blue lines) using a two-sample two-tailed $t$-test for the last 30 years of the time series. (From Balan Sarojini et al., 2012.) Results for the Climate Research Unit (CRU) TS3.1 data set are shown in Figure 10.A.2. are expressed as a percentage of climatological precipitation and that the observed and simulated changes are largely consistent between CMIP5 models and observations given data uncertainty. Use of additional data sets illustrates remaining observational uncertainty in high latitudes of the NH (Figure 10.11). Regional-scale attribution of precipitation change is still problematic although regional climate models have yielded simulations consistent with observed wintertime changes for northern Europe (Bhend and von Storch, 2008; Tapiador, 2010).

Precipitation change over ocean has been attributed to human influence by Fyfe et al. (2012) for the high-latitude SH in austral summer, where zonally averaged precipitation has declined around $45^{\circ} \mathrm{S}$ and increased around $60^{\circ} \mathrm{S}$ since 1957, consistent with CMIP5 historical simulations, with the magnitude of the half-century trend outside the range of simulated natural variability. Confidence in this attribution result, despite limitations in precipitation observations, is enhanced by its consistency with trends in large-scale sea level pressure data (see Section 10.3.3).

In summary, there is medium confidence that human influence has contributed to large-scale changes in precipitation patterns over land. The expected anthropogenic fingerprints of change in zonal mean precipitation-reductions in low latitudes and increases in $\mathrm{NH}$ mid to high latitudes-have been detected in annual and some seasonal data. Observational uncertainties including limited global coverage and large natural variability, in addition to challenges in precipitation modeling, limit confidence in assessment of climatic changes in precipitation.

\subsubsection{Changes in Surface Hydrologic Variables}

This subsection assesses recent research on detection and attribution of long-term changes in continental surface hydrologic variables, including soil moisture, evapotranspiration and streamflow. Streamflows are often subject to large non-climatic human influence, such as diversions and land use changes, that must be accounted for in order to attribute detected hydrologic changes to climate change. Cryospheric aspects of surface hydrology are discussed in Section 10.5; extremes in surface hydrology (such as drought) and precipitation are covered in Section 10.6.1. The variables discussed here are subject to large modeling uncertainties (Chapter 9) and observational challenges (Chapter 2), which in combination place severe limits on climate change detection and attribution.

Direct observational records of soil moisture and surface fluxes tend to be sparse and/or short, thus limiting recent assessments of change in these variables (Jung et al., 2010). Assimilated land surface data sets and new satellite observations (Chapter 2) are promising tools, but assessment of past and future climate change of these variables (Hoekema and Sridhar, 2011) is still generally carried out on derived quantities such as the Palmer Drought Severity Index, as discussed more fully in Section 10.6.1. Recent observations (Jung et al., 2010) show regional trends towards drier soils. An optimal detection analysis of reconstructed evapotranspiration identifies the effects of anthropogenic forcing on evapotranspiration, with the Centre National de Recherches Météorologiques (CNRM)-CM5 model simulating changes consistent with those estimated to have occurred (Douville et al., 2013). 

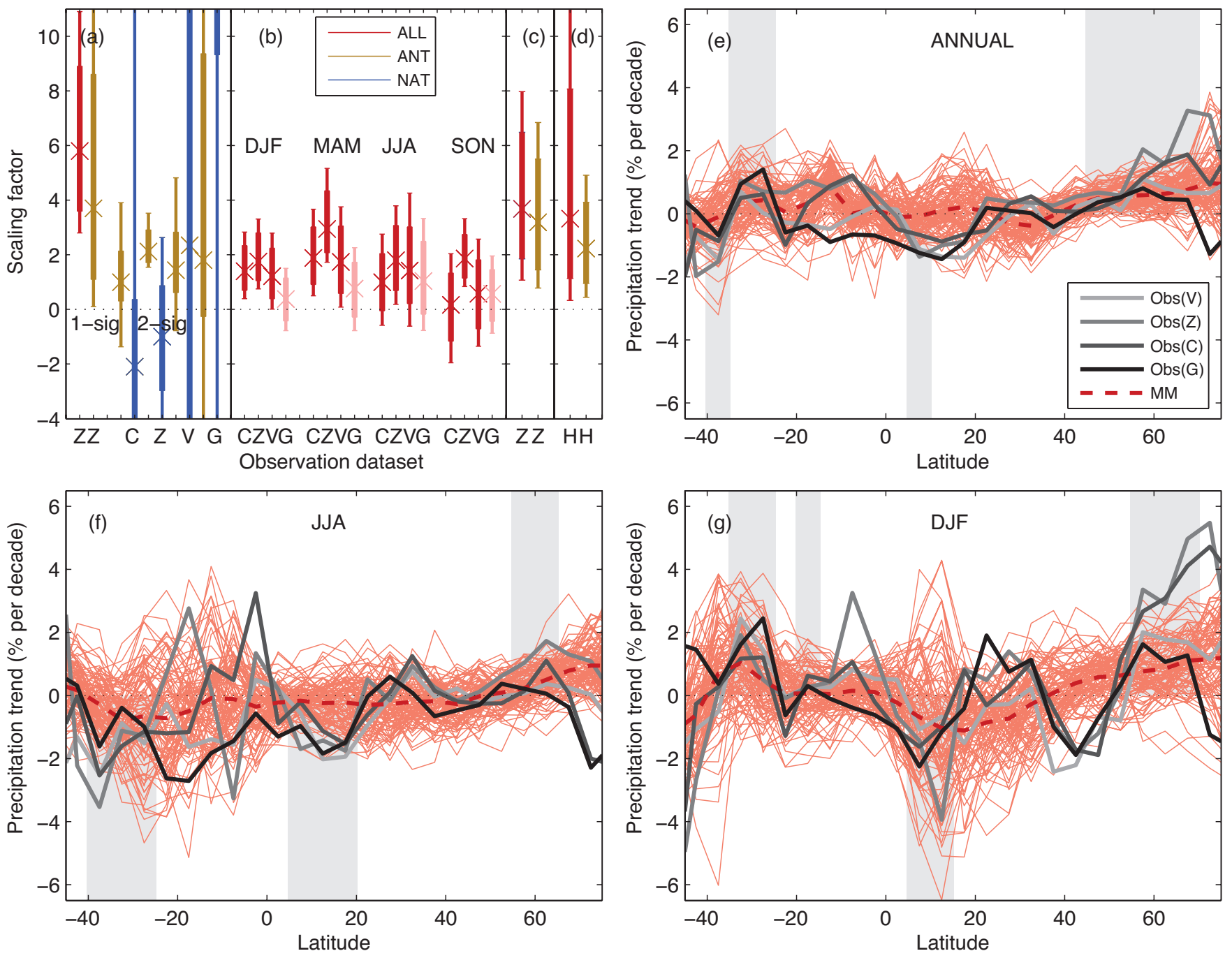

Figure 10.11 | Detection and attribution results for zonal land precipitation trends in the second half of the 20th century. (Top left) Scaling factors for precipitation changes. (Top right and bottom) Zonally averaged precipitation changes over continents from models and observations. (a) Crosses show the best-guess scaling factor derived from multi-model means. Thick bars show the 5 to $95 \%$ uncertainty range derived from model-simulated variability, and thin bars show the uncertainty range if doubling the multi-model variance. Red bars indicate scaling factors for the estimated response to all forcings, blue bars for natural-only forcing and brown bars for anthropogenic-only forcing. Labels on the $x$-axis identify results from four different observational data sets (Z is Zhang et al. (2007), C is Climate Research Unit (CRU), V is Variability Analyses of Surface Climate Observations (VasClim0), G is Global Precipitation Climatology Centre (GPCC), H is Hadley Centre gridded data set of temperature and precipitation extremes (HadEX)). (a) Detection and attribution results for annual averages, both single fingerprint ("1-sig"; 1950-1999) and two fingerprint results ("2-sig"; Z, C, G (1951-2005), V (1952-2000)). (b) Scaling factors resulting from single-fingerprint analyses for seasonally averaged precipitation (Z, C, G (1951-2005), V (1952-2000); the latter in pink as not designed for long-term homogeneity) for four different seasons. (c) Scaling factors for spatial pattern of Arctic precipitation trends (1951-1999). (d) Scaling factors for changes in large-scale intense precipitation (1951-1999). (e) Thick solid lines show observed zonally and annually averaged trends (\% per decade) for four different observed data sets. Corresponding results from individual simulations from 33 different climate models are shown as thin solid lines, with the multimodel mean shown as a red dashed line. Model results are masked to match the spatial and temporal coverage of the GPCC data set (denoted G in the seasonal scaling factor panel). Grey shading indicates latitude bands within which $>75 \%$ of simulations yield positive or negative trends. (f, g) Like (e) but showing zonally averaged precipitation changes for (f) June, July, August (JJA) and (g) December, January, February (DJF) seasons. Scaling factors (c) and (d) adapted from Min et al. (2008a) and Min et al. (2011), respectively; other results adapted from Zhang et al. (2007) and Polson et al. (2013).

Trends towards earlier timing of snowmelt-driven streamflows in western North America since 1950 have been demonstrated to be different from natural variability (Hidalgo et al., 2009). Similarly, internal variability associated with natural decade-scale fluctuations could not account for recent observed declines of northern Rocky Mountain streamflow (St Jacques et al., 2010). Statistical analyses of streamflows demonstrate regionally varying changes that are consistent with changes expected from increasing temperature, in Scandinavia (Wilson et al., 2010), Europe (Stahl et al., 2010) and the USA (Krakauer and Fung, 2008; Wang and Hejazi, 2011). Observed increases in Arctic river discharge, which could be a good integrator for monitoring changes in precipitation in high latitudes, are found to be explainable only if model simulations include anthropogenic forcings (Min et al., 2008a).

Barnett et al. (2008) analysed changes in the surface hydrology of the western USA, considering snow pack (measured as snow water equivalent), the seasonal timing of streamflow in major rivers, and average January to March daily minimum temperature over the region, the two hydrological variables they studied being closely related to temperature. Observed changes were compared with the output of a 
regional hydrologic model forced by the Parallel Climate Model (PCM) and Model for Interdisciplinary Research On Climate (MIROC) climate models. They derived a fingerprint of anthropogenic changes from the two climate models and found that the observations, when projected onto the fingerprint of anthropogenic changes, show a positive signal strength consistent with the model simulations that falls outside the range expected from internal variability as estimated from 1600 years of downscaled climate model data. They conclude that there is a detectable and attributable anthropogenic signature on the hydrology of this region.

In summary, there is medium confidence that human influence on climate has affected stream flow and evapotranspiration in limited regions of middle and high latitudes of the NH. Detection and attribution studies have been applied only to limited regions and using a few models. Observational uncertainties are large and in the case of evapotranspiration depend on reconstructions using land surface models.

\subsubsection{Atmospheric Circulation and Patterns of Variability}

The atmospheric circulation is driven by various processes including the uneven heating of the Earth's surface by solar radiation, land-sea contrast and orography. The circulation transports heat from warm to cold regions and thereby acts to reduce temperature contrasts. Thus, changes in circulation and in patterns of variability are of critical importance for the climate system, influencing regional climate and regional climate variability. Any such changes are important for local climate change because they could act to reinforce or counteract the effects of external forcings on climate in a particular region. Observed changes in atmospheric circulation and patterns of variability are assessed in Section 2.7.5. Although new and improved data sets are now available, changes in patterns of variability remain difficult to detect because of large variability on interannual to decadal time scales (Section 2.7).

Since AR4, progress has been made in understanding the causes of changes in circulation-related climate phenomena and modes of variability such as the width of the tropical circulation, and the Southern Annular Mode (SAM). For other climate phenomena, such as ENSO, Indian Ocean Dipole (IOD), Pacific Decadal Oscillation (PDO), and monsoons, there are large observational and modelling uncertainties (see Section 9.5 and Chapter 14), and there is low confidence that changes in these phenomena, if observed, can be attributed to human-induced influence.

\subsubsection{Tropical Circulation}

Various indicators of the width of the tropical belt based on independent data sets suggest that the tropical belt as a whole has widened since 1979; however, the magnitude of this change is very uncertain (Fu et al., 2006; Hudson et al., 2006; Hu and Fu, 2007; Seidel and Randel, 2007; Seidel et al., 2008; Lu et al., 2009; Fu and Lin, 2011; Hu et al., 2011; Davis and Rosenlof, 2012; Lucas et al., 2012; Wilcox et al., 2012; Nguyen et al., 2013) (Section 2.7.5). CMIP3 and CMIP5 simulations suggest that anthropogenic forcings have contributed to the observed widening of the tropical belt since 1979 (Johanson and Fu, 2009; Hu et al., 2013). On average the poleward expansion of the Hadley circulation and other indicators of the width of the tropical belt is greater than determined from CMIP3 and CMIP5 simulations (Seidel et al., 2008; Johanson and Fu, 2009; Hu et al., 2013; Figure 10.12). The causes as to why models underestimate the observed poleward expansion of the tropical belt are not fully understood. Potential factors are lack of understanding of the magnitude of natural variability as well as changes in observing systems that also affect reanalysis products (Thorne and Vose, 2010; Lucas et al., 2012; Box 2.3).

Climate model simulations suggest that Antarctic ozone depletion is a major factor in causing poleward expansion of the southern Hadley cell during austral summer over the last three to five decades with GHGs also playing a role (Son et al., 2008, 2009, 2010; McLandress et al., 2011; Polvani et al., 2011; Hu et al., 2013). In reanalysis data a detectable signal of ozone forcing is separable from other external forcing including GHGs when utilizing both CMIP5 and CMIP3 simulations combined (Min and Son, 2013). An analysis of CMIP3 simulations suggests that $\mathrm{BC}$ aerosols and tropospheric ozone were the main drivers of the observed poleward expansion of the northern Hadley cell in boreal summer (Allen et al., 2012). It is found that global greenhouse warming causes increase in static stability, such that the onset of baroclinicity is shifted poleward, leading to poleward expansion of the Hadley circulation (Frierson, 2006; Frierson et al., 2007; Hu and Fu, 2007; Lu et al., 2007, 2008). Tropical SST increase may also contribute to a widening of the Hadley circulation (Hu et al., 2011; Staten et al., 2012). Althoughe some Atmospheric General Circulation Model (AGCM) simulations forced by observed time-varying SSTs yield a widening by about $1^{\circ}$ in latitude over 1979-2002 (Hu et al., 2011), other simulations suggest that SST changes have little effect on the tropical expansion when based on the tropopause metric of the tropical width (Lu et al., 2009). However, it is found that the tropopause metric is not

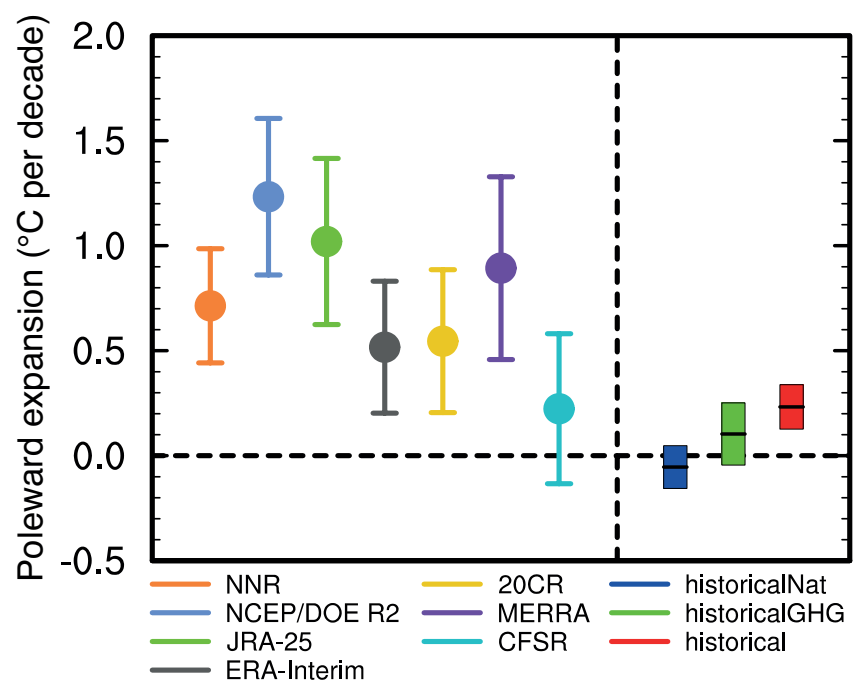

Figure 10.12 | December to February mean change of southern border of the Hadley circulation. Unit is degree in latitude per decade. Reanalysis data sets (see also Box 2.3) are marked with different colours. Trends are all calculated over the period of 1979 2005. The terms historicalNAT, historicalGHG, and historical denote CMIP5 simulations with natural forcing, with greenhouse gas forcing and with both anthropogenic and natural forcings, respectively. For each reanalysis data set, the error bars indicate the 95\% confidence level of the standard $t$-test. For CMIP5 simulations, trends are first calculated for each model, and all ensemble members of simulations are used. Then, trends are averaged for multi-model ensembles. Trend uncertainty is estimated from multi-model ensembles, as twice the standard error. (Updated from Hu et al., 2013.) 
very reliable because of the use of arbitrary thresholds (Birner, 2010; Davis and Rosenlof, 2012).

In summary, there are multiple lines of evidence that the Hadley cell and the tropical belt as a whole have widened since at least 1979; however, the magnitude of the widening is very uncertain. Based on modelling studies there is medium confidence that stratospheric ozone depletion has contributed to the observed poleward shift of the southern Hadley cell border during austral summer, with GHGs also playing a role. The contribution of internal climate variability to the observed poleward expansion of the Hadley circulation remains very uncertain.

\subsubsection{Northern Annular Mode/North Atlantic Oscillation}

The NAO, which exhibited a positive trend from the 1960s to the 1990s, has since exhibited lower values, with exceptionally low anomalies in the winters of 2009/2010 and 2010/2011 (Section 2.7.8). This means that the positive trend in the NAO discussed in the AR4 has considerably weakened when evaluated up to 2011. Similar results apply to the closely related Northern Annular Mode (NAM), with its upward trend over the past 60 years in the 20th Century Reanalysis (Compo et al., 2011) and in Hadley Centre Sea Level Pressure data set 2r (HadSLP2r; Allan and Ansell, 2006) not being significant compared to internal variability (Figure 10.13). An analysis of CMIP5 models shows that they simulate positive trends in NAM in the DJF season over this period, albeit not as large as those observed which are still within the range of natural internal variability (Figure 10.13).

Other work (Woollings, 2008) demonstrates that while the NAM is largely barotropic in structure, the simulated response to anthropogenic forcing has a strong baroclinic component, with an opposite geopotential height trends in the mid-troposphere compared to the surface in many models. Thus while the circulation response to anthropogenic forcing may project onto the NAM, it is not entirely captured by the NAM index.

Consistent with previous findings (Hegerl et al., 2007b), Gillett and Fyfe (2013) find that GHGs tend to drive a positive NAM response in the CMIP5 models. Recent modelling work also indicates that ozone changes drive a small positive NAM response in spring (Morgenstern et al., 2010; Gillett and Fyfe, 2013).

\subsubsection{Southern Annular Mode}

The Southern Annular Mode (SAM) index has remained mainly positive since the publication of the AR4, although it has not been as strongly positive as in the late 1990s. Nonetheless, an index of the SAM shows a significant positive trend in most seasons and data sets over the 1951-2011 period (Figure 10.13; Table 2.14). Recent modelling studies confirm earlier findings that the increase in GHG concentrations tends to lead to a strengthening and poleward shift of the SH eddy-driven polar jet (Karpechko et al., 2008; Son et al., 2008, 2010; Sigmond et al., 2011; Staten et al., 2012; Swart and Fyfe, 2012; Eyring et al., 2013; Gillett and Fyfe, 2013) which projects onto the positive phase of the SAM. Stratospheric ozone depletion also induces a strengthening and poleward shift of the polar jet in models, with the largest response in austral summer (Karpechko et al., 2008; Son et al., 2008, 2010; McLandress
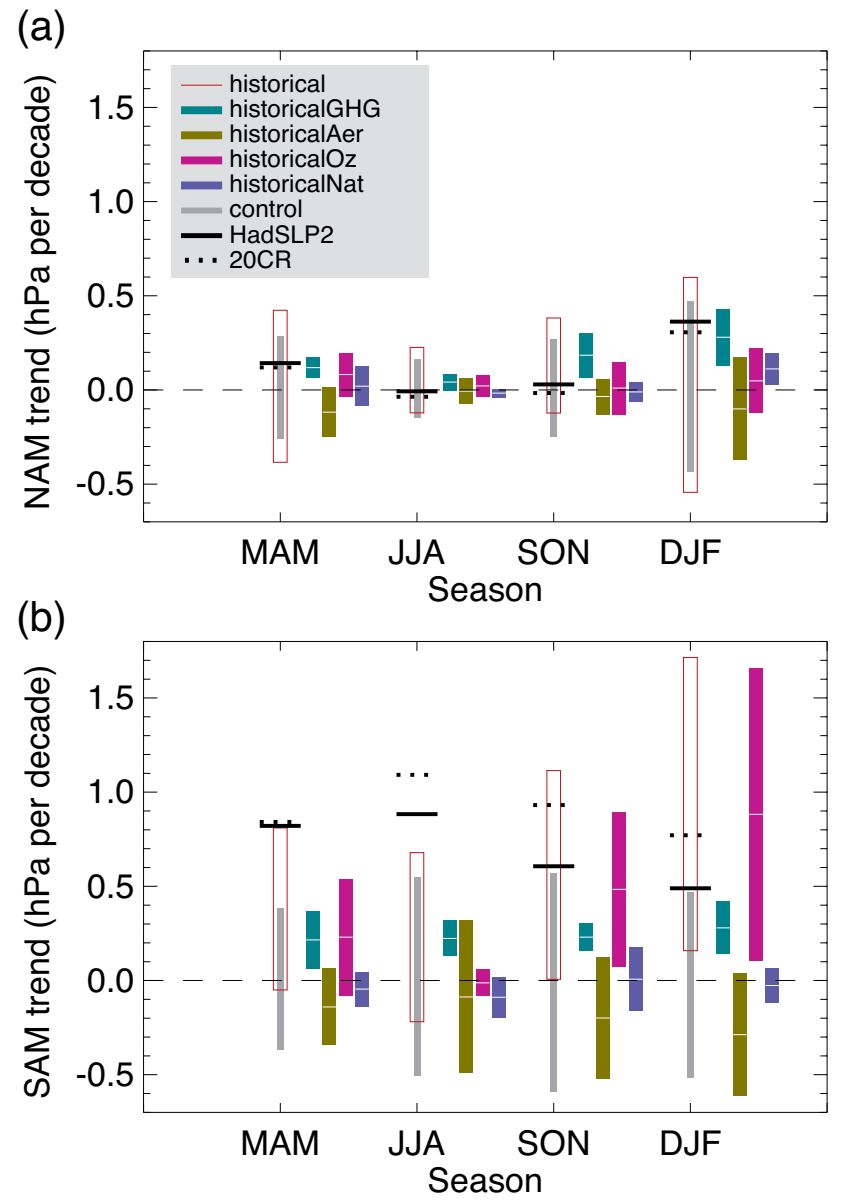

Figure 10.13 | Simulated and observed 1951-2011 trends in the Northern Annular Mode (NAM) index (a) and Southern Annular Mode (SAM) index (b) by season. The NAM is a Li and Wang (2003) index based on the difference between zonal mean seal level pressure (SLP) at $35^{\circ} \mathrm{N}$ and $65^{\circ} \mathrm{N}$. and the, and the SAM index is a difference between zonal mean SLP at $40^{\circ} \mathrm{S}$ and $65^{\circ} \mathrm{S}$ (Gong and Wang, 1999). Both indices are defined without normalization, so that the magnitudes of simulated and observed trends can be compared. Black lines show observed trends from the HadSLP2r data set (Allan and Ansell, 2006) (solid), and the 20th Century Reanalysis (Compo et al., 2011) (dotted). Grey bars and red boxes show 5 to $95 \%$ ranges of trends in CMIP5 control and historical simulations respectively. Ensemble mean trends and their 5 to $95 \%$ uncertainties are shown for the response to greenhouse gases (light green), aerosols (dark green), ozone (magenta) and natural (blue) forcing changes, based on CMIP5 individual forcing simulations. (Adapted from Gillett and Fyfe, 2013.)

et al., 2011; Polvani et al., 2011; Sigmond et al., 2011; Gillett and Fyfe, 2013). Sigmond et al. (2011) find approximately equal contributions to simulated annual mean SAM trends from GHGs and stratospheric ozone depletion up to the present. Fogt et al. (2009) demonstrate that observed SAM trends over the period 1957-2005 are positive in all seasons, but only statistically significant in DJF and March, April, May (MAM), based on simulated internal variability. Roscoe and Haigh (2007) apply a regression-based approach and find that stratospheric ozone changes are the primary driver of observed trends in the SAM. Observed trends are also consistent with CMIP3 simulations including stratospheric ozone changes in all seasons, though in MAM observed trends are roughly twice as large as those simulated (Miller et al., 2006). Broadly consistent results are found when comparing observed trends and CMIP5 simulations (Figure 10.13), with a station-based SAM index showing a significant positive trend in MAM, JJA and DJF, compared 
to simulated internal variability over the 1951-2010 period. Fogt et al. (2009) find that the largest forced response has likely occurred in DJF, the season in which stratospheric ozone depletion has been the dominant contributor to the observed trends.

Taking these findings together, it is likely that the positive trend in the SAM seen in austral summer since the mid-20th century is due in part to stratospheric ozone depletion. There is medium confidence that GHGs have also played a role.

\subsubsection{Change in Global Sea Level Pressure Patterns}

A number of studies have applied formal detection and attribution studies to global fields of atmospheric SLP finding detection of human influence on global patterns of SLP (Gillett et al., 2003, 2005; Gillett and Stott, 2009). Analysing the contributions of different forcings to observed changes in SLP, Gillett and Stott (2009) find separately detectable influences of anthropogenic and natural forcings in zonal mean seasonal mean SLP, strengthening evidence for a human influence on SLP. Based on the robustness of the evidence from multiple models we conclude that it is likely that human influence has altered SLP patterns globally since 1951 .

\subsection{Changes in Ocean Properties}

This section assesses the causes of oceanic changes in the main properties of interest for climate change: ocean heat content, ocean salinity and freshwater fluxes, sea level, oxygen and ocean acidification.

\subsubsection{Ocean Temperature and Heat Content}

The oceans are a key part of the Earth's energy balance (Boxes 3.1 and 13.1). Observational studies continue to demonstrate that the ocean heat content has increased in the upper layers of the ocean during the second half of the 20th century and early 21st century (Section 3.2; Bindoff et al., 2007), and that this increase is consistent with a net positive radiative imbalance in the climate system. It is of significance that this heat content increase is an order of magnitude larger than the increase in energy content of any other component of the Earth's ocean-atmosphere-cryosphere system and accounts for more than $90 \%$ of the Earth's energy increase between 1971 and 2010 (e.g., Boxes 3.1 and 13.1; Bindoff et al., 2007; Church et al., 2011; Hansen et al., 2011).

Despite the evidence for anthropogenic warming of the ocean, the level of confidence in the conclusions of the AR4 report-that the warming of the upper several hundred meters of the ocean during the second half of the 20th century was likely to be due to anthropogenic forcing-reflected the level of uncertainties at that time. The major uncertainty was an apparently large decadal variability (warming in the 1970s and cooling in the early 1980s) in the observational estimates that was not simulated by climate models (Hegerl et al., 2007b, see their Table 9.4). The large decadal variability in observations raised concerns about the capacity of climate models to simulate observed variability. There were also lingering concerns about the presence of non-climate-related biases in the observations of ocean heat content change (Gregory et al., 2004; AchutaRao et al., 2006). After the IPCC AR4 report in 2007, time-and depth-dependent systematic errors in bathythermograph temperatures were discovered (Gouretski and Koltermann, 2007; Section 3.2). Bathythermograph data account for a large fraction of the historical temperature observations and are therefore a source of bias in ocean heat content studies. Bias corrections were then developed and applied to observations. With the newer bias-corrected estimates (Domingues et al., 2008; Wijffels et al., 2008; Ishii and Kimoto, 2009; Levitus et al., 2009), it became obvious that the large decadal variability in earlier estimates of global upper-ocean heat content was an observational artefact (Section 3.2).

The interannual to decadal variability of ocean temperature simulated by the CMIP3 models agrees better with observations when the model data is sampled using the observational data mask (AchutaRao et al., 2007). In the upper 700 m, CMIP3 model simulations agreed more closely with observational estimates of global ocean heat content based on bias-corrected ocean temperature data, both in terms of the decadal variability and multi-decadal trend (Figure 10.14a) when forced with the most complete set of natural and anthropogenic forcings (Domingues et al., 2008). For the simulations with the most complete set of forcings, the multi-model ensemble mean trend was only $10 \%$ smaller than observed for 1961-1999. Model simulations that included only anthropogenic forcing (i.e., no solar or volcanic forcing) significantly overestimate the multi-decadal trend and underestimate decadal variability. This overestimate of the trend is partially caused by the ocean's response to volcanic eruptions, which results in rapid cooling followed by decadal or longer time variations during the recovery phase. Although it has been suggested (Gregory, 2010) that the cooling trend from successive volcanic events is an artefact because models were not spun up with volcanic forcing, this discrepancy is not expected to be as significant in the upper ocean as in the deeper layers where longer term adjustments take place (Gregory et al., 2012 ). Thus for the upper ocean, there is high confidence that the more frequent eruptions during the second half of the 20th century have caused a multi-decadal cooling that partially offsets the anthropogenic warming and contributes to the apparent decadal variability (Church et al., 2005; Delworth et al., 2005; Fyfe, 2006; Gleckler et al., 2006; Gregory et al., 2006; AchutaRao et al., 2007; Domingues et al., 2008; Palmer et al., 2009; Stenchikov et al., 2009).

Gleckler et al. (2012) examined the detection and attribution of upperocean warming in the context of uncertainties in the underlying observational data sets, models and methods. Using three bias-corrected observational estimates of upper-ocean temperature changes (Domingues et al., 2008; Ishii and Kimoto, 2009; Levitus et al., 2009) and models from the CMIP3 multi-model archive, they found that multi-decadal trends in the observations were best understood by including contributions from both natural and anthropogenic forcings. The anthropogenic fingerprint in observed upper-ocean warming, driven by global mean and basin-scale pattern changes, was also detected. The strength of this signal (estimated from successively longer trend periods of ocean heat content starting from 1970) crossed the 5\% and $1 \%$ significance threshold in 1980 and progressively becomes more strongly detected for longer trend periods (Figure 10.14b), for all ocean heat content time series. This stronger detection for longer periods occurs because the noise (standard deviation of trends in the unforced chang- 
es in pattern similarity from model control runs) tends to decrease for longer trend lengths. On decadal time scales, there is limited evidence that basin scale space-time variability structure of CMIP3 models is approximately $25 \%$ lower than the (poorly constrained) observations, this underestimate is far less than the factor of 2 needed to throw the anthropogenic fingerprint into question. This result is robust to a number of known observational, model, methodological and structural uncertainties.
An analysis of upper-ocean ( 0 to $700 \mathrm{~m}$ ) temperature changes for 1955-2004, using bias-corrected observations and 20 global climate models from CMIP5 (Pierce et al., 2012) builds on previous detection and attribution studies of ocean temperature (Barnett et al., 2001, 2005; Pierce et al., 2006). This analysis found that observed temperature changes during the above period are inconsistent with the effects of natural climate variability. That is signal strengths are separated from zero at the $5 \%$ significance level, and the probability that the

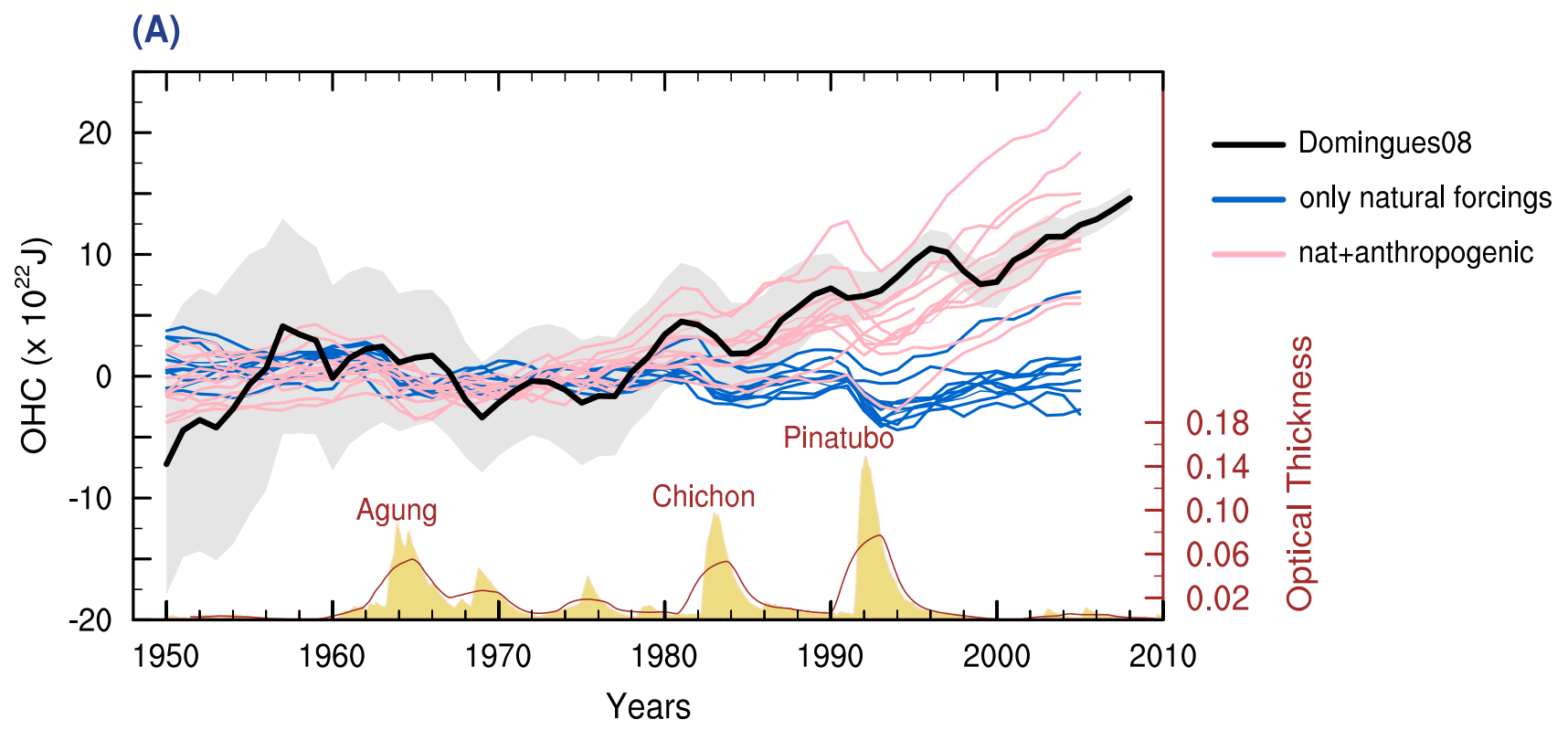

(B)

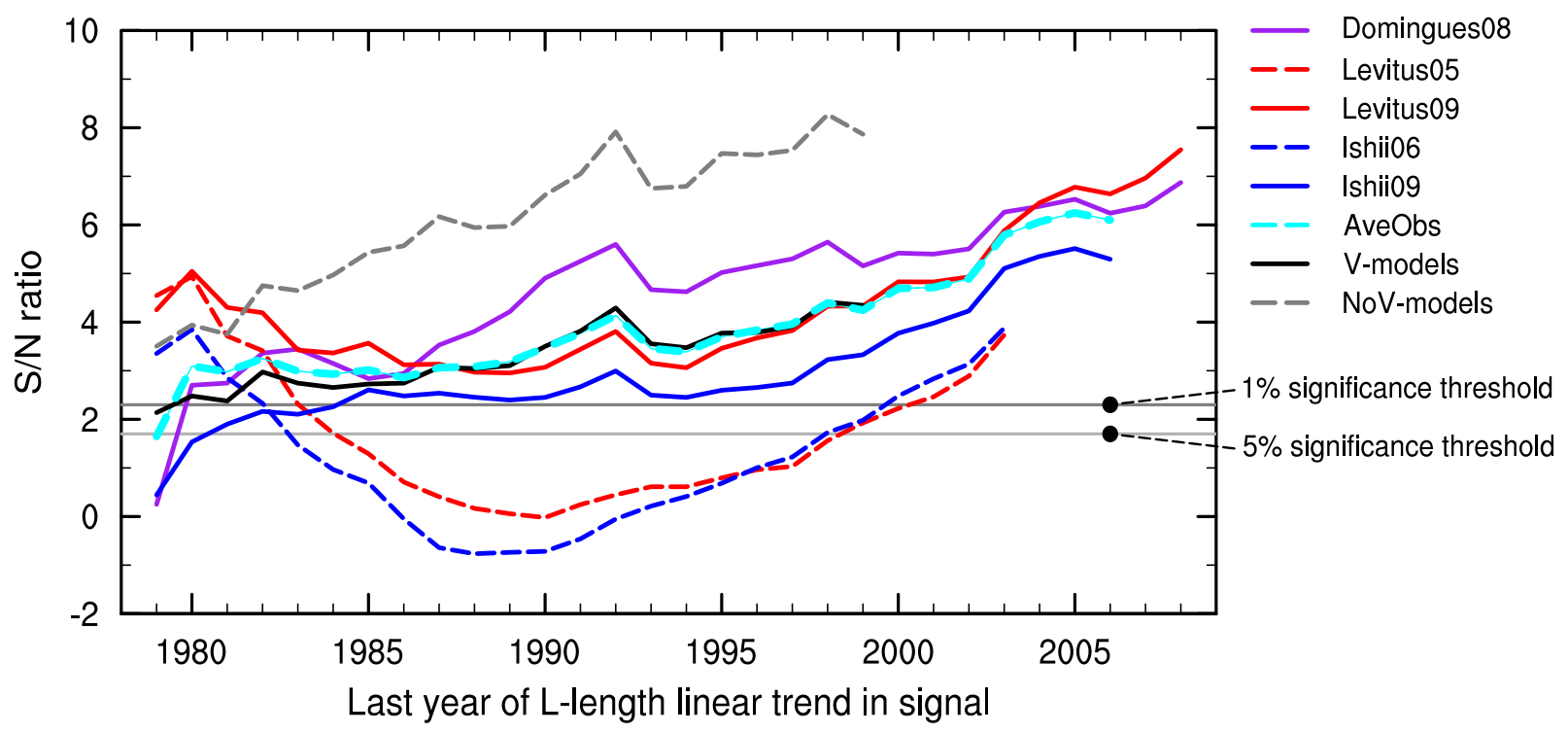

Figure 10.14 | (A) Comparison of observed global ocean heat content for the upper $700 \mathrm{~m}$ (updated from Domingues et al. 2008) with simulations from ten CMIP5 models that included only natural forcings ('HistoricalNat' runs shown in blue lines) and simulations that included natural and anthropogenic forcings ('Historical' runs in pink lines). Grey shading shows observational uncertainty. The global mean stratospheric optical depth (Sato et al., 1993) in beige at the bottom indicates the major volcanic eruptions and the brown curve is a 3-year running average of these values. (B) Signal-to-noise (S/N) ratio (plotted as a function of increasing trend length $\mathrm{L}$ ) of basin-scale changes in volume averaged temperature of newer, expendable bathythermograph (XBT)-corrected data (solid red, purple and blue lines), older, uncorrected data (dashed red and blue lines); the average of the three corrected observational sets (AveObs; dashed cyan line); and simulations that include volcanic (V) or exclude volcanic eruptions (NoV) (black solid and grey dashed lines respectively). The start date for the calculation of signal trends is 1970 and the initial trend length is 10 years. The $1 \%$ and $5 \%$ significance thresholds are shown (as horizontal grey lines) and assume a Gaussian distribution of noise trends in the V-models control-run pseudo-principal components. The detection time is defined as the year at which $\mathrm{S} / \mathrm{N}$ exceeds and remains above $1 \%$ or $5 \%$ significance threshold (Gleckler et al., 2012). 
null hypothesis of observed changes being consistent with natural variability is less than 0.05 from variability either internal to the climate system alone, or externally forced by solar fluctuations and volcanic eruptions. However, the observed ocean changes are consistent with those expected from anthropogenically induced atmospheric changes from GHGs and aerosol concentrations.

Attribution to anthropogenic warming from recent detection and attribution studies (Gleckler et al., 2012; Pierce et al., 2012) have made use of new bias-corrected observations and have systematically explored methodological uncertainties, yielding more confidence in the results. With greater consistency and agreement across observational data sets and resolution of structural issues, the major uncertainties at the time of AR4 have now largely been resolved. The high levels of confidence and the increased understanding of the contributions from both natural and anthropogenic sources across the many studies mean that it is very likely that the increase in global ocean heat content observed in the upper $700 \mathrm{~m}$ since the 1970s has a substantial contribution from anthropogenic forcing.

Although there is high confidence in understanding the causes of global heat content increases, attribution of regional heat content changes are less certain. Earlier regional studies used a fixed depth data and only considered basin-scale averages (Barnett et al., 2005). At regional scales, however, changes in advection of ocean heat are important and need to be isolated from changes due to air-sea heat fluxes (Palmer et al., 2009; Grist et al., 2010). Their fixed isotherm (rather than fixed depth) approach to optimal detection analysis, in addition to being largely insensitive to observational biases, is designed to separate the ocean's response to air-sea flux changes from advective changes. Airsea fluxes are the primary mechanism by which the oceans are expected to respond to externally forced anthropogenic and natural volcanic influences. The finer temporal resolution of the analysis allowed Palmer et al. (2009) to attribute distinct short-lived cooling episodes to major volcanic eruptions while, at multi-decadal time scales, a more spatially uniform near-surface ( upper $200 \mathrm{~m}$ ) warming pattern was detected across all ocean basins (except in high latitudes where the isotherm approach has limitations due to outcropping of isotherms at the ocean surface) and attributed to anthropogenic causes at the $5 \%$ significance level. Considering that individual ocean basins are affected by different observational and modelling uncertainties and that internal variability is larger at smaller scales, detection of significant anthropogenic forcing through space and time studies (Palmer et al., 2009; Pierce et al., 2012) provides more compelling evidence of human influence at regional scales of near-surface ocean warming observed during the second half of the 20th century.

\subsubsection{Ocean Salinity and Freshwater Fluxes}

There is increasing recognition of the importance of ocean salinity as an essential climate variable (Doherty et al., 2009), particularly for understanding the hydrological cycle. In the IPCC Fourth Assessment Report observed ocean salinity change indicated that there was a systematic pattern of increased salinity in the shallow subtropics and a tendency to freshening of waters that originate in the polar regions (Bindoff et al., 2007; Hegerl et al., 2007b) (Figure 10.15a, upper and lower panels). New atlases and revisions of the earlier work based on the increasing number of the Array for Real-time Geostrophic Oceanography (ARGO) profile data, and historical data have extended the observational salinity data sets allowing the examination of the longterm changes at the surface and in the interior of the ocean (Section 3.3) and supporting analyses of precipitation changes over land (see Sections 10.3.2.2 and 2.5.1).

Patterns of subsurface salinity changes largely follow the existing mean salinity pattern at the surface and within the ocean. For example, the inter-basin contrast between the Atlantic (salty) and Pacific Oceans (fresh) has intensified over the observed record (Boyer et al., 2005; Hosoda et al., 2009; Roemmich and Gilson, 2009; von Schuckmann et al., 2009; Durack and Wijffels, 2010). In the Southern Ocean, many studies show a coherent freshening of Antarctic Intermediate Water that is subducted at about $50^{\circ} \mathrm{S}$ (Johnson and Orsi, 1997; Wong et al., 1999; Bindoff and McDougall, 2000; Curry et al., 2003; Boyer et al., 2005; Roemmich and Gilson, 2009; Durack and Wijffels, 2010; Helm et al., 2010; Kobayashi et al., 2012). There is also a clear increase in salinity of the high-salinity subtropical waters (Durack and Wijffels, 2010; Helm et al., 2010).

The 50-year trends in surface salinity show that there is a strong positive correlation between the mean climate of the surface salinity and its temporal changes from 1950 to 2000 (see Figures 3.4 and $10.15 b$ 'ocean obs' point). The correlation between the climate and the trends in surface salinity of 0.7 implies that fresh surface waters get fresher, and salty waters get saltier (Durack et al., 2012). Such patterns of surface salinity change are also found in Atmosphere-Ocean General Circulation Models (AOGCM) simulations both for the 20th century and projected future changes into the 21st century (Figure 10.15b). The pattern of temporal change in observations from CMIP3 simulations is particularly strong for those projections using Special Report on Emission Scenarios (SRES) with larger global warming changes (Figure 10.15b). For the period 1950-2000 the observed amplification of the surface salinity is $16 \pm 10 \%$ per ${ }^{\circ} \mathrm{C}$ of warming and is twice the simulated surface salinity change in CMIP3 models. This difference between the surface salinity amplification is plausibly caused by the tendency of CMIP3 ocean models mixing surface salinity into deeper layers and consequently surface salinity increases at a slower rate than observed (Durack et al., 2012).

Although there are now many established observed long-term trends of salinity change at the ocean surface and within the interior ocean at regional and global scales (Section 3.3), there are relatively few studies that attribute these changes formally to anthropogenic forcing. Analysis at the regional scale of the observed recent surface salinity increases in the North Atlantic $\left(20^{\circ} \mathrm{N}\right.$ to $\left.50^{\circ} \mathrm{N}\right)$ show a small signal that could be attributed to anthropogenic forcings but for this ocean is not significant compared with internal variability (Stott et al., 2008a; Terray et al., 2012; and Figure 10.15c). On a larger spatial scale, the surface salinity patterns in the band from $30^{\circ} \mathrm{S}$ to $50^{\circ} \mathrm{N}$ show anthropogenic contributions that are larger than the 5 to $95 \%$ uncertainty range (Terray et al., 2012). The strongest signals that can be attributed to anthropogenic forcing are in the tropics (TRO, $30^{\circ} \mathrm{S}$ to $30^{\circ} \mathrm{N}$ ) and the western Pacific. These results also show the salinity contrast between the Pacific and Atlantic oceans is also enhanced with significant contributions from anthropogenic forcing. 
(A)

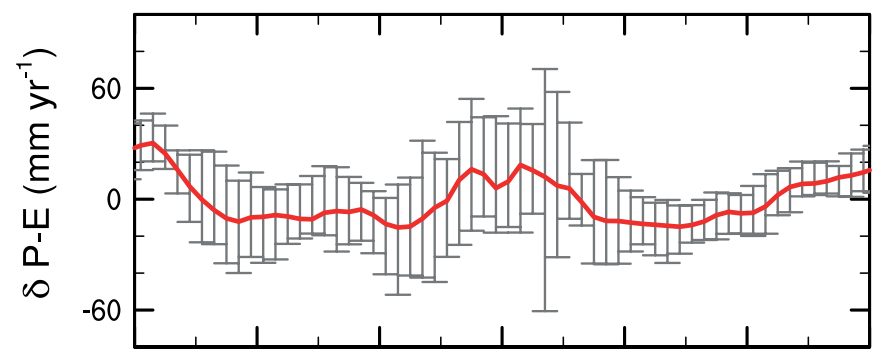

10

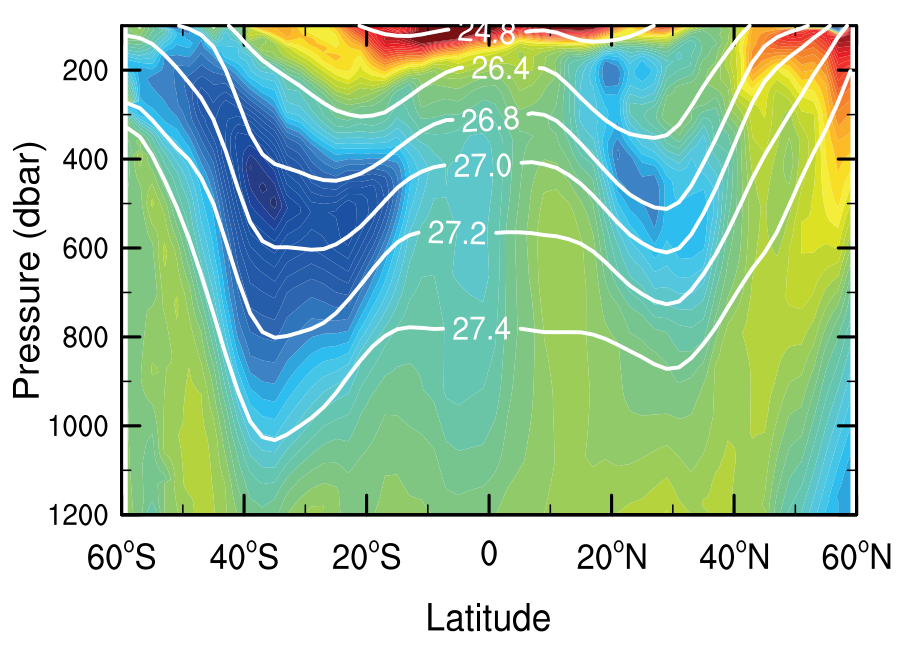

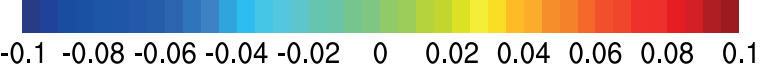
Change in salinity (psu)
(B)

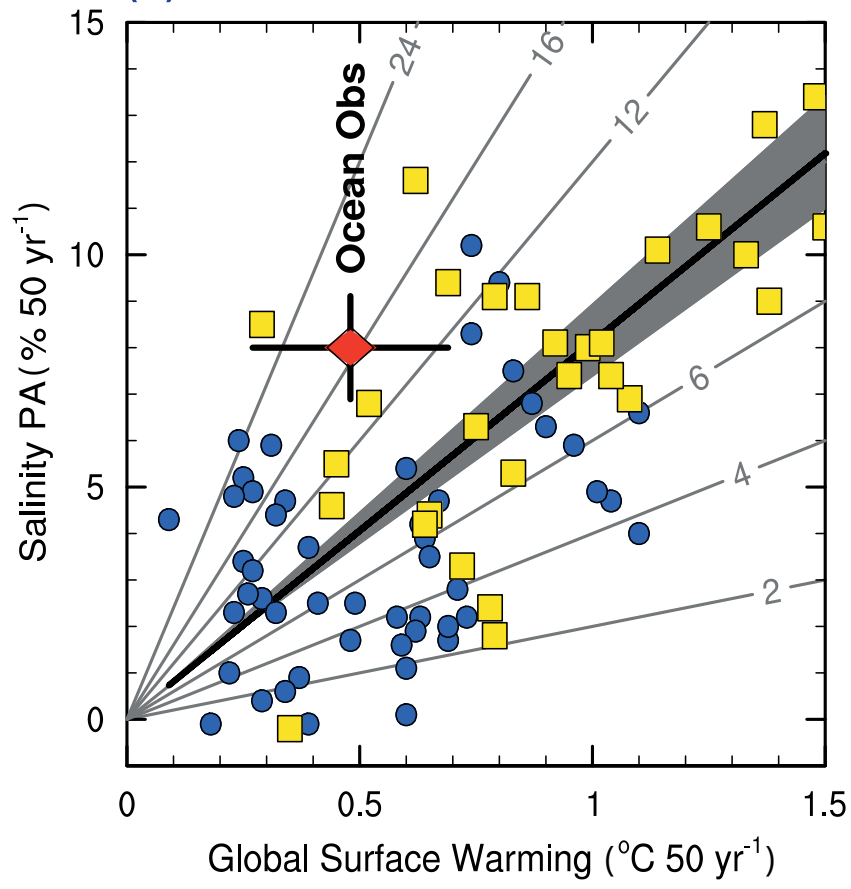

$20^{\text {th }}$ Century

Scenarios (A2, A1B, B1)

ocean obs (Durack \& Wijffels, 2010)

(C)

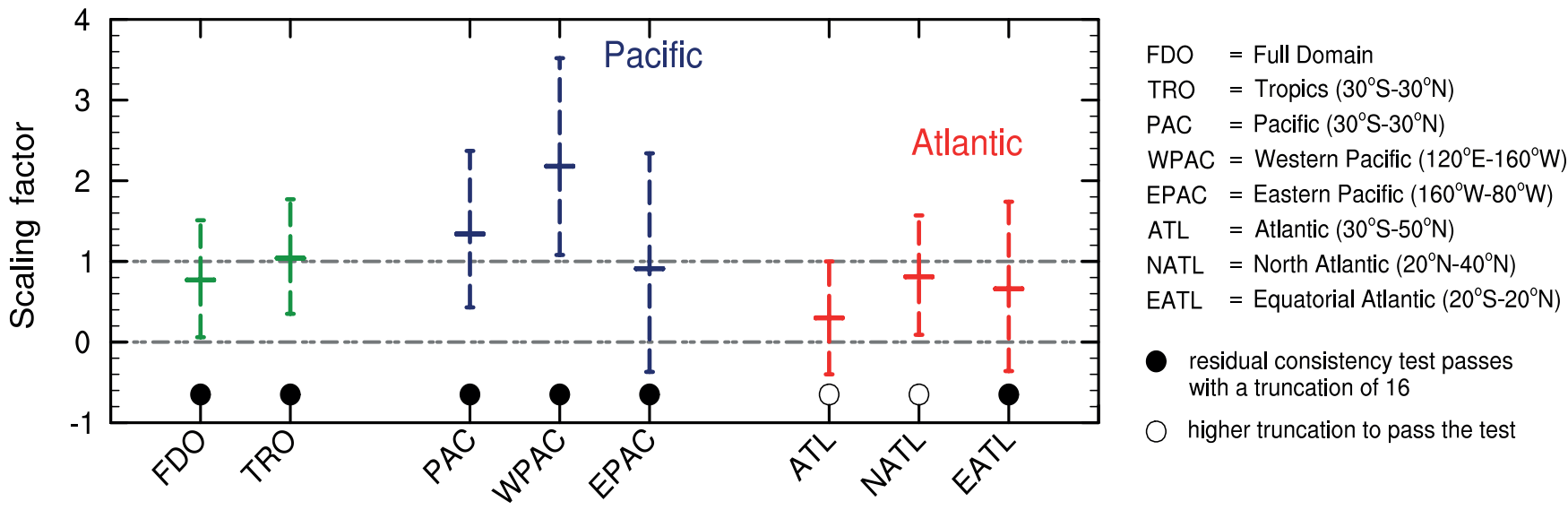

Figure 10.15 | Ocean salinity change and hydrologic cycle. (A) Ocean salinity change observed in the interior of the ocean (A, lower panel in practical salinity units or psu, and white lines are surfaces of constant density) and comparison with ten CMIP3 model projections of precipitation minus evaporation $\delta(P-E)$ in $\mathrm{mm}_{\mathrm{yr}} \mathrm{r}^{-1}$ for the same period as the observed changes (1970 to 1990s) (A, top panel, red line is the mean of the simulations and error bars are the simulated range). (B) The amplification of the current surface salinity pattern over a 50-year period as a function of global temperature change. Ocean surface salinity pattern amplification has a 16\% increase for the 1950-2000 period (red diamond, see text and Section 3.3). Also on this panel CMIP3 simulations from Special Report on Emission Scenarios (SRES) (yellow squares) and from 20th century simulations (blue circles). A total of 93 simulations have been used. (C) Regional detection and attribution in the equatorial Pacific and Atlantic Oceans for 1970 to 2002 . Scaling factors for all forcings (anthropogenic) fingerprint are shown (see Box 10.1) with their 5 to $95 \%$ uncertainty range, estimated using the total least square approach. Full domain (FDO, $30^{\circ} \mathrm{S}$ to $50^{\circ} \mathrm{N}$ ), Tropics (TRO, $30^{\circ} \mathrm{S}$ to $30^{\circ} \mathrm{N}$ ), Pacific (PAC, $30^{\circ} \mathrm{S}$ to $30^{\circ} \mathrm{N}$ ), west Pacific (WPAC, $120^{\circ} \mathrm{E}$ to $160^{\circ} \mathrm{W}$ ), east Pacific (EPAC, $160^{\circ} \mathrm{W}$ to $80^{\circ} \mathrm{W}$ ), Atlantic (ATL, $30^{\circ} \mathrm{S}$ to $50^{\circ} \mathrm{N}$ ), subtropical north Atlantic (NATL, $20^{\circ} \mathrm{N}$ to $40^{\circ} \mathrm{N}$ ) and equatorial Atlantic (EATL, $20^{\circ} \mathrm{S}$ to $20^{\circ} \mathrm{N}$ ) factors are shown. Black filled dots indicate when the residual consistency test passes with a truncation of 16 whereas empty circles indicate a higher truncation was needed to pass the consistency test. Horizontal dashed lines indicate scaling factor of 0 or 1 . (A, B and C are adapted from Helm et al. (2010), Durack et al. (2012) and Terray et al. (2012), respectively.) 
On a global scale surface and subsurface salinity changes (1955-2004) over the upper $250 \mathrm{~m}$ of the water column cannot be explained by natural variability (probability is $<0.05$ ) (Pierce et al., 2012). However, the observed salinity changes match the model distribution of forced changes (GHG and tropospheric aerosols), with the observations typically falling between the 25th and 75th percentile of the model distribution at all depth levels for salinity (and temperature). Natural external variability taken from the simulations with just solar and volcanic variations in forcing do not match the observations at all, thus excluding the hypothesis that observed trends can be explained by just solar or volcanic variations.

The results from surface salinity trends and changes are consistent with the results from studies of precipitation over the tropical ocean from the shorter satellite record (Wentz et al., 2007; Allan et al., 2010). These surface salinity results are also consistent with our understanding of the thermodynamic response of the atmosphere to warming (Held and Soden, 2006; Stephens and $\mathrm{Hu}, 2010$ ) and the amplification of the water cycle. The large number of studies showing patterns of change consistent with amplification of the water cycle, and the detection and attribution studies for the tropical oceans (Terray et al., 2012) and the global pattern of ocean salinity change (Pierce et al., 2012), when combined with our understanding of the physics of the water cycle and estimates of internal climate variability, give high confidence in our understanding of the drivers of surface and near surface salinity changes. It is very likely that these salinity changes have a discernable contribution from anthropogenic forcing since the 1960s.

\subsubsection{Sea Level}

At the time of the AR4, the historical sea level rise budget had not been closed (within uncertainties), and there were few studies quantifying the contribution of anthropogenic forcing to the observed sea level rise and glacier melting. Relying on expert assessment, the AR4 had concluded based on modelling and ocean heat content studies that ocean warming and glacier mass loss had very likely contributed to sea level rise during the latter half of the 20th century. The AR4 had reported that climate models that included anthropogenic and natural forcings simulated the observed thermal expansion since 1961 reasonably well, and that it is very unlikely that the warming during the past half century is due only to known natural causes (Hegerl et al., 2007b).

Since the AR4, corrections applied to instrumental errors in ocean temperature measurements have considerably improved estimates of upper-ocean heat content (see Sections 3.2 and 10.4.1), and therefore ocean thermal expansion. Closure of the global mean sea level rise budget as an evolving time series since the early1970s (Church et al., 2011) indicates that the two major contributions to the rate of global mean sea level rise have been thermal expansion and glacier melting with additional contributions from Greenland and Antarctic ice sheets. Observations since 1971 indicate with high confidence that thermal expansion and glaciers (excluding the glaciers in Antarctica) explain $75 \%$ of the observed rise (see Section 13.3.6). Ice sheet contributions remain the greatest source of uncertainty over this period and on longer time scales. Over the 20th century, the global mean sea level rise budget (Gregory et al., 2012 ) has been another important step in understanding the relative contributions of different drivers.
The observed contribution from thermal expansion is well captured in climate model simulations with historical forcings as are contributions from glacier melt when simulated by glacier models driven by climate model simulations of historical climate (Church et al., 2013; Table 13.1). The model results indicate that most of the variation in the contributions of thermal expansion and glacier melt to global mean sea level is in response to natural and anthropogenic RFs (Domingues et al., 2008; Palmer et al., 2009; Church et al., 2013).

The strong physical relationship between thermosteric sea level and ocean heat content (through the equation of state for seawater) means that the anthropogenic ocean warming (Section 10.4.1) has contributed to global sea level rise over this period through thermal expansion. As Section 10.5.2 concludes, it is likely that the observed substantial mass loss of glaciers is due to human influence and that it is likely that anthropogenic forcing and internal variability are both contributors to recent observed changes on the Greenland ice sheet. The causes of recently observed Antarctic ice sheet contribution to sea level are less clear due to the short observational record and incomplete understanding of natural variability. Taking the causes of Greenland ice sheet melt and glacier mass loss together (see Section 10.5.2), it is concluded with high confidence that it is likely that anthropogenic forcing has contributed to sea level rise from melting glaciers and ice sheets. Combining the evidence from ocean warming and mass loss of glaciers we conclude that it is very likely that there is a substantial contribution from anthropogenic forcing to the global mean sea level rise since the 1970s.

On ocean basin scales, detection and attribution studies do show the emergence of detectable signals in the thermosteric component of sea level that can be largely attributed to human influence (Barnett et al., 2005; Pierce et al., 2012). Regional changes in sea level at the subocean basin scales and finer exhibit more complex variations associated with natural (dynamical) modes of climate variability (Section 13.6). In some regions, sea level trends have been observed to differ significantly from global mean trends. These have been related to thermosteric changes in some areas and in others to changing wind fields and resulting changes in the ocean circulation (Han et al., 2010; Timmermann et al., 2010; Merrifield and Maltrud, 2011). The regional variability on decadal and longer time scales can be quite large (and is not well quantified in currently available observations) compared to secular changes in the winds that influence sea level. Detection of human influences on sea level at the regional scale (that is smaller than sub-ocean basin scales) is currently limited by the relatively small anthropogenic contributions compared to natural variability (Meyssignac et al., 2012) and the need for more sophisticated approaches than currently available.

\subsubsection{Oxygen and Ocean Acidity}

Oxygen is an important physical and biological tracer in the ocean (Section 3.8.3) and is projected to decline by 3 to $6 \%$ by 2100 in response to surface warming (see Section 6.4.5). Oxygen decreases are also observed in the atmosphere and linked to burning of fossil fuels (Section 6.1.3.2). Despite the relatively few observational studies of oxygen change in the oceans (Bindoff and McDougall, 2000; Ono et al., 2001; Keeling and Garcia, 2002; Emerson et al., 2004; Aoki et al., 2005; 
Mecking et al., 2006; Nakanowatari et al., 2007; Brandt et al., 2010) they all show a pattern of change consistent with the known ocean circulation and surface ventilation. A recent global analysis of oxygen data from the 1960s to 1990s for change confirm these earlier results and extends the spatial coverage from local to global scales (Helm et al., 2011). The strongest decreases in oxygen occur in the mid-latitudes of both hemispheres, near regions where there is strong water renewal and exchange between the ocean interior and surface waters. The attribution study of oxygen decreases using two Earth System Models (ESMs) concluded that observed changes for the Atlantic Ocean are 'indistinguishable from natural internal variability'; however, the changes of the global zonal mean to external forcing (all forcings including GHGs) has a detectable influence at the $10 \%$ significance level (Andrews et al., 2013). The chief sources of uncertainty are the paucity of oxygen observations, particularly in time, the precise role of the biological pump and changes in ocean productivity in the models (see Sections 3.8.3 and 6.4.5), and model circulation biases particularly near the oxygen minimum zone in tropical waters (Brandt et al., 2010; Keeling et al., 2010; Stramma et al., 2010). These results of observed changes in oxygen and the attribution studies of oxygen changes (Andrews et al., 2013), along with the attribution of human influences on the physical factors that affect oxygen in the oceans such as surface temperatures changes (Section 10.3.2), increased ocean heat content (Section 10.4.1) and observed increased in ocean stratification (Section 3.2.2) provides evidence for human influence on oxygen. When these lines of evidence are taken together it is concluded that with medium confidence or about as likely as not that the observed oxygen decreases can be attributed in part to human influences.

The observed trends (since the 1980s) for ocean acidification and its cause from rising $\mathrm{CO}_{2}$ concentrations is discussed in Section 3.8.2 (Box 3.2 and Table 10.1). There is very high confidence that anthropogenic $\mathrm{CO}_{2}$ has resulted in the acidification of surface waters of between -0.0015 and $-0.0024 \mathrm{pH}$ units per year.

\subsection{Cryosphere}

This section considers changes in sea ice, ice sheets and ice shelves, glaciers, snow cover. The assessment of attribution of human influences on temperature over the Arctic and Antarctica is in Section 10.3.1.

\subsubsection{Sea Ice}

\subsubsection{Arctic and Antarctic Sea Ice}

The Arctic cryosphere shows large observed changes over the last decade as noted in Chapter 4 and many of these shifts are indicators of major regional and global feedback processes (Kattsov et al., 2010). An assessment of sea ice models' capacity to simulate Arctic and Antarctic sea ice extent is given in Section 9.4.3. Of principal importance is 'Arctic Amplification' (see Box 5.1) where surface temperatures in the Arctic are increasing faster than elsewhere in the world.

The rate of decline of Arctic sea ice thickness and September sea ice extent has increased considerably in the first decade of the 21st century (Maslanik et al., 2007; Nghiem et al., 2007; Comiso and Nishio,
2008; Deser and Teng, 2008; Zhang et al., 2008; Alekseev et al., 2009; Comiso, 2012; Polyakov et al., 2012). Based on a sea ice reanalysis and verified by ice thickness estimates from satellite sensors, it is estimated that three quarters of summer Arctic sea ice volume has been lost since the 1980s (Schweiger et al., 2011; Maslowski et al., 2012; Laxon et al., 2013; Overland and Wang, 2013). There was also a rapid reduction in ice extent, to $37 \%$ less in September 2007 and to $49 \%$ less in September 2012 relative to the 1979-2000 climatology (Figure 4.11, Section 4.2.2). Unlike the loss record set in 2007 that was dominated by a major shift in climatological winds, sea ice loss in 2012 was more due to a general thinning of the sea ice (Lindsay et al., 2009; Wang et al., 2009a; Zhang et al., 2013). All recent years have ice extents that fall at least two standard deviations below the long-term sea ice trend.

The amount of old, thick multi-year sea ice in the Arctic has decreased by 50\% from 2005 through 2012 (Giles et al., 2008; Kwok et al., 2009; Kwok and Untersteiner, 2011 and Figures 4.13 and 4.14). Sea ice has also become more mobile (Gascard et al., 2008). We now have seven years of data that show sea ice conditions are substantially different to that observed prior to 2006. The relatively large increase in the percentage of first year sea ice across the Arctic basin can be considered 'a new normal.'

Confidence in detection of change comes in part from the consistency of multiple lines of evidence. Since AR4, evidence has continued to accumulate from a range of observational studies that systematic changes are occurring in the Arctic. Persistent trends in many Arctic variables, including sea ice, the timing of spring snow melt, increased shrubbiness in tundra regions, changes in permafrost, increased area of forest fires, changes in ecosystems, as well as Arctic-wide increases in air temperatures, can no longer be associated solely with the dominant climate variability patterns such as the Arctic Oscillation, Pacific North American pattern or Atlantic Meridional Oscillation (AMO) (Quadrelli and Wallace, 2004; Vorosmarty et al., 2008; Overland, 2009; Brown and Robinson, 2011; Mahajan et al., 2011; Oza et al., 2011a; Wassmann et al., 2011; Nagato and Tanaka, 2012). Duarte et al. (2012) completed a meta-analysis showing evidence from multiple indicators of detectable climate change signals in the Arctic.

The increase in the magnitude of recent Arctic temperature and decrease in sea ice volume and extent are hypothesized to be due to coupled Arctic amplification mechanisms (Serreze and Francis, 2006; Miller et al., 2010). These feedbacks in the Arctic climate system suggest that the Arctic is sensitive to external forcing (Mahlstein and Knutti, 2012 ). Historically, changes were damped by the rapid formation of sea ice in autumn causing a negative feedback and a rapid seasonal cooling. But recently, the increased mobility and loss of multiyear sea ice, combined with enhanced heat storage in the sea ice-free regions of the Arctic Ocean form a connected set of processes with positive feedbacks causing an increase in Arctic temperatures and a decrease in sea ice extent (Manabe and Wetherald, 1975; Gascard et al., 2008; Serreze et al., 2009; Stroeve et al., 2012a, 2012b) . In addition to the well known ice albedo feedback where decreased sea ice cover decreases the amount of insolation reflected from the surface, there is a late summer/early autumn positive ice insulation feedback due to additional ocean heat storage in areas previously covered by sea ice 
(Jackson et al., 2010). Arctic amplification may also have a contribution from poleward heat transport in the atmosphere and ocean (Langen and Alexeev, 2007; Graversen and Wang, 2009; Doscher et al., 2010; Yang et al., 2010).

It appears that recent Arctic changes are in response to a combination of global-scale warming, from warm anomalies from internal climate variability on different time scales, and are amplified from the multiple feedbacks described above. For example, when the 2007 sea ice minimum occurred, Arctic temperatures had been rising and sea ice extent had been decreasing over the previous two decades (Stroeve et al., 2008; Screen and Simmonds, 2010). Nevertheless, it took unusually persistent southerly winds along the dateline over the summer months to initiate the sea ice loss event in 2007 (Zhang et al., 2008; Wang et al., 2009b). Similar southerly wind patterns in previous years did not initiate major reductions in sea ice extent because the sea ice was too thick to respond (Overland et al., 2008). Increased oceanic heat transport through the Barents Sea in the first decade of the 21st century and the AMO on longer time scales may also have played a role in determining sea ice anomalies in the Atlantic Arctic (Dickson et al., 2000; Semenov, 2008; Zhang et al., 2008; Day et al., 2012) . Based on the evidence in the previous paragraphs there is high confidence that these Arctic amplification mechanisms are currently affecting regional Arctic climate. But it also suggests that the timing of future major sea ice loss events will be difficult to project. There is evidence therefore that internal variability of climate, long-term warming, and Arctic Amplification feedbacks have all contributed to recent decreases in Arctic sea ice (Kay et al., 2011b; Kinnard et al., 2011; Overland et al., 2011; Notz and Marotzke, 2012).

Turning to model-based attribution studies, Min et al. (2008b) compared the seasonal evolution of Arctic sea ice extent from observations with those simulated by multiple General Circulation Models (GCMs) for 1953-2006. Comparing changes in both the amplitude and shape of the annual cycle of the sea ice extent reduces the chance of spurious detection due to coincidental agreement between the response to anthropogenic forcing and other factors, such as slow internal variability. They found that human influence on the sea ice extent changes has been robustly detected since the early 1990s. The anthropogenic signal is also detectable for individual months from May to December, suggesting that human influence, strongest in late summer, now also extends into colder seasons. Kay et al. (2011b), Jahn et al. (2012) and Schweiger et al. (2011) used the Community Climate System Model 4 (CCSM4) to investigate the influence of anthropogenic forcing on late 20th century and early 21st century Arctic sea ice extent and volume trends. On all time scales examined ( 2 to $50+$ years), the most extreme negative extent trends observed in the late 20th century cannot be explained by modeled internal variability alone. Comparing trends from the CCSM4 ensemble to observed trends suggests that internal variability could account for approximately half of the observed 1979-2005 September Arctic sea ice extent loss. Attribution of anthropogenic forcing is also shown by comparing September sea ice extent as projected by seven models from the set of CMIP5 models' hindcasts to control runs without anthropogenic forcing (Figure 10.16a; Wang and Overland, 2009). The mean of sea ice extents in seven models' ensemble members are below the level of their control runs by about 1995, similar to the result of Min et al. (2008b).
A question as recently as 6 years ago was whether the recent Arctic warming and sea ice loss was unique in the instrumental record and whether the observed trend would continue (Serreze et al., 2007). Arctic temperature anomalies in the 1930s were apparently as large as those in the 1990s and 2000s. There is still considerable discussion of the ultimate causes of the warm temperature anomalies that occurred in the Arctic in the 1920s and 1930s (Ahlmann, 1948; Veryard, 1963; Hegerl et al., 2007a, 2007b). The early 20th century warm period, while reflected in the hemispheric average air temperature record (Brohan et al., 2006), did not appear consistently in the mid-latitudes nor on the Pacific side of the Arctic (Johannessen et al., 2004; Wood and Overland, 2010). Polyakov et al. (2003) argued that the Arctic air temperature records reflected a natural cycle of about 50 to 80 years. However, many authors (Bengtsson et al., 2004; Grant et al., 2009; Wood and Overland, 2010; Brönnimann et al., 2012) instead link the 1930s temperatures to internal variability in the North Atlantic atmospheric and ocean circulation as a single episode that was sustained by ocean and sea ice processes in the Arctic and north Atlantic. The Arctic-wide increases of temperature in the last decade contrast with the episodic regional increases in the early 20th century, suggesting that it is unlikely that recent increases are due to the same primary climate process as the early 20 th century.

In the case of the Arctic we have high confidence in observations since 1979, from models (see Section 9.4.3 and from simulations comparing with and without anthropogenic forcing), and from physical understanding of the dominant processes; taking these three factors together it is very likely that anthropogenic forcing has contributed to the observed decreases in Arctic sea ice since 1979.

Whereas sea ice extent in the Arctic has decreased, sea ice extent in the Antarctic has very likely increased (Section 4.2.3). Sea ice extent across the $\mathrm{SH}$ over the year as a whole increased by 1.3 to $1.67 \%$ per decade from 1979 to 2012, with the largest increase in the Ross Sea during the autumn, while sea ice extent decreased in the Amundsen-Bellingshausen Sea (Comiso and Nishio, 2008; Turner et al., 2009, 2013; Section 4.2.3; Oza et al., 2011b). The observed upward trend in Antarctic sea ice extent is found to be inconsistent with internal variability based on the residuals from a linear trend fitted to the observations, though this approach could underestimate multi-decadal variability (Section 4.2.3; Turner et al., 2013; Section 4.2.3; Zunz et al., 2013). The CMIP5 simulations on average simulate a decrease in Antarctic sea ice extent (Turner et al., 2013; Zunz et al., 2013; Figure 10.16b), though Turner et al. (2013) find that approximately $10 \%$ of CMIP5 simulations exhibit an increasing trend in Antarctic sea ice extent larger than observed over the 1979-2005 period. However, Antarctic sea ice extent variability appears on average to be too large in the CMIP5 models (Turner et al., 2013; Zunz et al., 2013). Overall, the shortness of the observed record and differences in simulated and observed variability preclude an assessment of whether or not the observed increase in Antarctic sea ice extent is inconsistent with internal variability. Based on Figure $10.16 \mathrm{~b}$ and Meehl et al. (2007b), the trend of Antarctic sea ice loss in simulations due to changes in forcing is weak (relative to the Arctic) and the internal variability is high, and thus the time necessary for detection is longer than in the Arctic. 
Several recent studies have investigated the possible causes of Antarctic sea ice trends. Early studies suggested that stratospheric ozone depletion may have driven increasing trends in Antarctic ice extent (Goosse et al., 2009; Turner et al., 2009; WMO (World Meteorological Organization), 2011), but recent studies demonstrate that simulated sea ice extent decreases in response to prescribed changes in stratospheric ozone (Sigmond and Fyfe, 2010; Bitz and Polvani, 2012). An alternative explanation for the lack of melting of Antarctic sea ice is that sub-surface ocean warming, and enhanced freshwater input possibly in part from ice shelf melting, have made the high-latitude Southern Ocean fresher (see Section 3.3) and more stratified, decreasing the upward heat flux and driving more sea ice formation (Zhang, 2007; Goosse et al., 2009; Bintanja et al., 2013). An idealized simulation of the response to freshwater input similar to that estimate due to ice shelf melting exhibited an increase in sea ice extent (Bintanja et al., 2013), but this result has yet to be reproduced with other models. Overall we conclude that there is low confidence in the scientific understanding of the observed increase in Antarctic sea ice extent since 1979, owing to
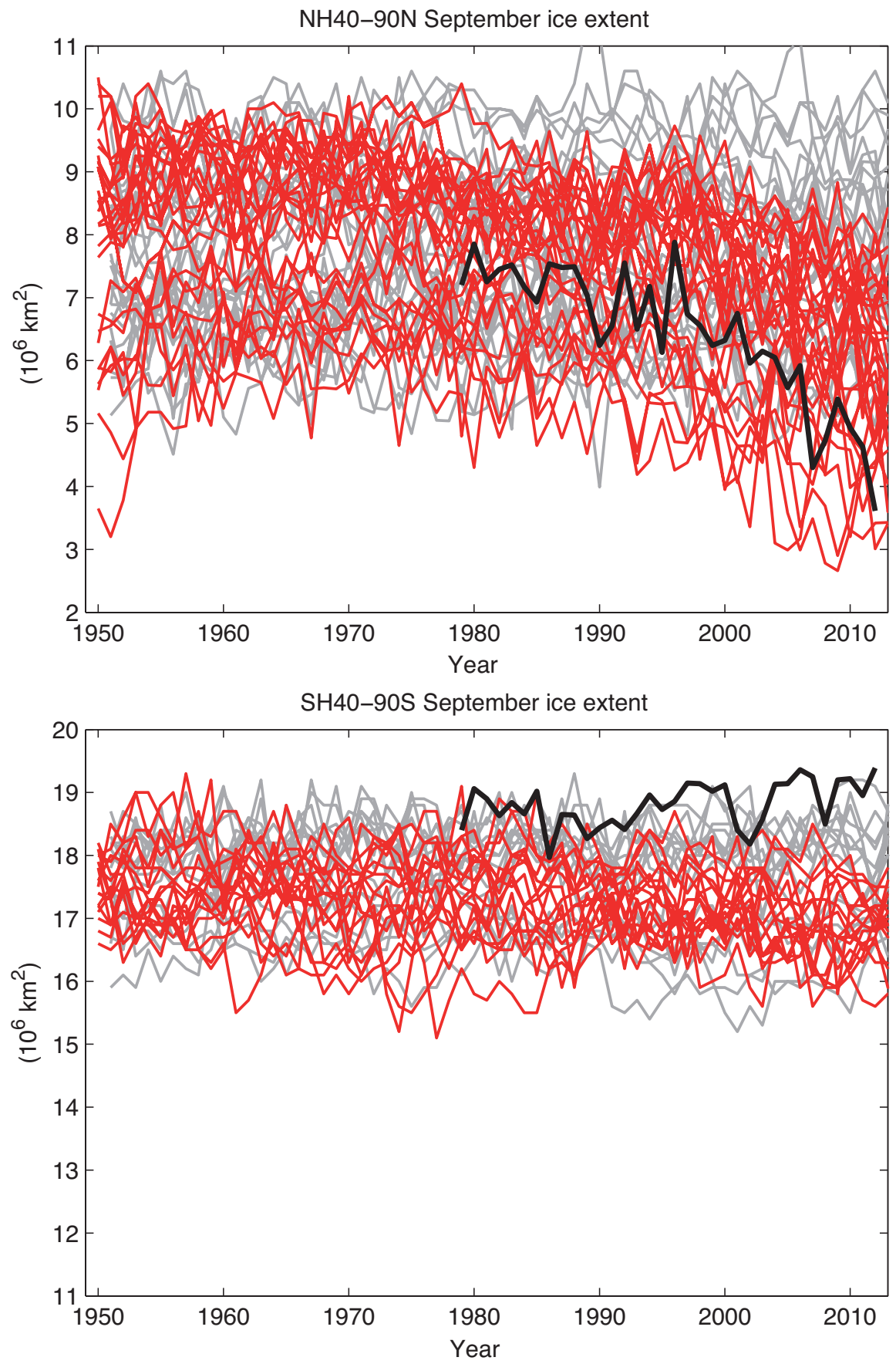

Figure 10.16 | September sea ice extent for Arctic (top) and Antarctic (bottom) adapted from (Wang and Overland, 2012). Only CMIP5 models that simulated seasonal mean and magnitude of seasonal cycle in reasonable agreement with observations are included in the plot. The grey lines are the runs from the pre-industrial control runs, and the red lines are from Historical simulations runs patched with RCP8.5 runs for the period of 2005-2012. The black line is based on data from National Snow and Ice Data Center (NSIDC). There are 24 ensemble members from 11 models for the Arctic and 21 members from 6 models for the Antarctic plot. See Supplementary Material for the precise models used in the top and bottom panel. 
the larger differences between sea ice simulations from CMIP5 models and to the incomplete and competing scientific explanations for the causes of change and low confidence in estimates of internal variability (Section 9.4.3).

\subsubsection{Ice Sheets, Ice Shelves and Glaciers}

\subsubsection{Greenland and Antarctic Ice Sheets}

The Greenland and Antarctic ice sheets are important to regional and global climate because (along with other cryospheric elements) they cause a polar amplification of surface temperatures, a source of fresh water to the ocean, and represent a source of potentially irreversible change to the state of the Earth system (Hansen and Lebedeff, 1987). These two ice sheets are important contributors to sea level rise representing two-thirds of the contributions from all ice covered regions (Jacob et al., 2012; Pritchard et al., 2012; see Sections 4.4 and 13.3.3). Observations of surface mass balance (increased ablation versus increased snowfall) are dealt with in Section 4.4 .3 and ice sheet models are discussed in Sections 13.3 and 13.5.

Attribution of change is difficult as ice sheet and glacier changes are local and ice sheet processes are not generally well represented in climate models thus precluding formal single-step detection and attribution studies. However, Greenland observational records show large recent changes. Section 13.3 concludes that regional models for Greenland can reproduce trends in the surface mass balance loss quite well if they are forced with the observed meteorological record, but not with forcings from a Global Climate Model. Regional model simulations (Fettweis et al., 2013) show that Greenland surface melt increases nonlinearly with rising temperatures due to the positive feedback between surface albedo and melt.

There have been exceptional changes in Greenland since 2007 marked by record-setting high air temperatures, ice loss by melting and marine-terminating glacier area loss (Hanna et al., 2013; Section 4.4. 4). Along Greenland's west coast temperatures in 2010 and 2011 were the warmest since record keeping began in 1873 resulting in the highest observed melt rates in this region since 1958 (Fettweis et al., 2011). The annual rate of area loss in marine-terminating glaciers was 3.4 times that of the previous 8 years, when regular observations became available. In 2012, a new record for summertime ice mass loss was two standard deviations below the 2003-2012 mean, as estimated from the Gravity Recovery and Climate Experiment (GRACE) satellite (Tedesco et al., 2012). The trend of summer mass change during 2003-2012 is rather uniform over this period at $-29 \pm 11 \mathrm{Gt} \mathrm{yr}^{-1}$.

Record surface melts during 2007-2012 summers are linked to persistent atmospheric circulation that favored warm air advection over Greenland. These persistent events have changed in frequency since the beginning of the 2000s (L'Heureux et al., 2010; Fettweis et al., 2011). Hanna et al. (2013) show a weak relation of Greenland temperatures and ice sheet runoff with the AMO; they more strongly correlate with a Greenland atmospheric blocking index. Overland et al. (2012) and Francis and Vavrus (2012) suggest that the increased frequency of the Greenland blocking pattern is related to broader scale Arctic changes. Since 2007, internal variability is likely to have further enhanced the melt over Greenland. Mass loss and melt is also occurring in Greenland through the intrusion of warm water into the major glaciers such as Jacobshaven Glacier (Holland et al., 2008; Walker et al., 2009).

Hanna et al. (2008) attribute increased Greenland runoff and melt since 1990 to global warming; southern Greenland coastal and NH summer temperatures were uncorrelated between the 1960s and early 1990s but correlated significantly positively thereafter. This relationship was modulated by the NAO, whose summer index significantly negatively correlated with southern Greenland summer temperatures until the early 1990s but not thereafter. Regional modelling and observations tell a consistent story of the response of Greenland temperatures and ice sheet runoff to shifts in recent regional atmospheric circulation associated with larger scale flow patterns and global temperature increases. It is likely that anthropogenic forcing has contributed to surface melting of the Greenland ice sheet since 1993.

There is clear evidence that the West Antarctic ice sheet is contributing to sea level rise (Bromwich et al., 2013). Estimates of ice mass in Antarctic since 2000 show that the greatest losses are at the edges (see Section 4.4). An analysis of observations underneath a floating ice shelf off West Antarctica shows that ocean warming and more transport of heat by ocean circulation are largely responsible for increasing melt rates (Jacobs et al., 2011; Joughin and Alley, 2011; Mankoff et al., 2012; Pritchard et al., 2012).

Antarctica has regionally dependent decadal variability in surface temperature with variations in these trends depending on the strength of the SAM climate pattern. Recent warming in continental west Antarctica has been linked to SST changes in the tropical Pacific (Ding et al., 2011). As with Antarctic sea ice, changes in Antarctic ice sheets have complex causes (Section 4.4.3). The observational record of Antarctic mass loss is short and the internal variability of the ice sheet is poorly understood. Due to a low level of scientific understanding there is low confidence in attributing the causes of the observed loss of mass from the Antarctic ice sheet since 1993. Possible future instabilities in the west Antarctic ice sheet cannot be ruled out, but projection of future climate changes over West Antarctica remains subject to considerable uncertainty (Steig and Orsi, 2013).

\subsubsection{Glaciers}

In the 20th century, there is robust evidence that large-scale internal climate variability governs interannual to decadal variability in glacier mass (Hodge et al., 1998; Nesje et al., 2000; Vuille et al., 2008; Huss et al., 2010; Marzeion and Nesje, 2012) and, along with glacier dynamics, impacts glacier length as well (Chinn et al., 2005). On time periods longer than years and decades, there is now evidence of recent ice loss (see Section 4.3.3) due to increased ambient temperatures and associated regional moisture changes. However, few studies evaluate the direct attribution of the current observed mass loss to anthropogenic forcing, owing to the difficulty associated with contrasting scales between glaciers and the large-scale atmospheric circulation (Mölg et al., 2012). Reichert et al. (2002) show for two sample sites at mid and high latitude that internal climate variability over multiple millennia as represented in a GCM would not result in such short glacier lengths as observed in the 20th century. For a sample site at low latitude using 
multi-step attribution, Mölg et al. (2009) (and references therein) found a close relation between glacier mass loss and the externally forced atmosphere-ocean circulation in the Indian Ocean since the late 19th century. A second, larger group of studies makes use of century-scale glacier records (mostly glacier length but mass balance as well) to extract evidence for external drivers. These include local and regional changes in precipitation and air temperature, and related parameters (such as melt factors and solid/liquid precipitation ratio) estimated from the observed change in glaciers. In general these studies show that the glacier changes reveal unique departures since the 1970s, and that the inferred climatic drivers in the 20th century and particularly in most recent decades, exceed the variability of the earlier parts of the records (Oerlemans, 2005; Yamaguchi et al., 2008; Huss and Bauder, 2009; Huss et al., 2010; Leclercq and Oerlemans, 2011). These results underline the contrast to former centuries where observed glacier fluctuations can be explained by internal climate variability (Reichert et al., 2002; Roe and O'Neal, 2009; Nussbaumer and Zumbühl, 2012). Anthropogenic land cover change is an unresolved forcing, but a first assessment suggests that it does not confound the impacts of recent temperature and precipitation changes if the land cover changes are of local nature (Mölg et al., 2012). The robustness of the estimates of observed mass loss since the 1960s (Section 4.3, Figure 4.11), the confidence we have in estimates of natural variations and internal variability from long-term glacier records, and our understanding of glacier response to climatic drivers provides robust evidence and, therefore, high confidence that a substantial part of the mass loss of glaciers is likely due to human influence.

\subsubsection{Snow Cover}

Both satellite and in situ observations show significant reductions in the NH snow cover extent (SCE) over the past 90 years, with most reduction occurring in the 1980s (see Section 4.5). Formal detection and attribution studies have indicated anthropogenic influence on $\mathrm{NH}$ SCE (Rupp et al., 2013) and western USA snow water equivalent (SWE, Pierce et al., 2008). Pierce et al. (2008) detected anthropogenic influence in the ratio of 1 April SWE over October to March precipitation over the period 1950-1999. These reductions could not be explained by natural internal climate variability alone, nor by changes in solar and volcanic forcing. In their analysis of NH SCE using 13 CMIP5 simulations over the 1922-2005 period, Rupp et al. (2013) showed that some CMIP5 simulations with natural external and anthropogenic forcings could explain the observed decrease in spring SEC though the CMIP5 simulations with all forcing as a whole could only explain half of the magnitude of decrease, and that volcanic and solar variations (from four CMIP5 simulations) were inconsistent with observations. We conclude with high confidence in the observational and modelling evidence that the decrease in NH snow extent since the 1970s is likely to be caused by all external forcings and has an anthropogenic contribution (see Table 10.1).

\subsection{Extremes}

Because many of the impacts of climate changes may manifest themselves through weather and climate extremes, there is increasing interest in quantifying the role of human and other external influences on those extremes. SREX assessed causes of changes in different types of extremes including temperature and precipitation, phenomena that influence the occurrence of extremes (e.g., storms, tropical cyclones), and impacts on the natural physical environment such as drought (Seneviratne et al., 2012). This section assesses current understanding of causes of changes in weather and climate extremes, using AR4 as a starting point. Any changes or modifications to SREX assessment are highlighted.

\subsubsection{Attribution of Changes in Frequency/ Occurrence and Intensity of Extremes}

This sub-section assesses attribution of changes in the characteristics of extremes including frequency and intensity of extremes. Many of the extremes discussed in this sub-section are moderate extreme events that occur more than once in a year (see Box 2.4 for detailed discussion). Attribution of changes in the risk of specific extreme events, which are also very rare in general, is assessed in the next sub-section.

\subsubsection{Temperature Extremes}

AR4 concluded that 'surface temperature extremes have likely been affected by anthropogenic forcing'. Many indicators of climate extremes and variability showed changes consistent with warming, including a widespread reduction in number of frost days in mid-latitude regions and evidence that in many regions warm extremes had become warmer and cold extremes had become less cold. We next assess new studies made since AR4.

Relatively warm seasonal mean temperatures (e.g., those that have a recurrence once in 10 years) have seen a rapid increase in frequency for many regions worldwide (Jones et al., 2008; Stott et al., 2011; Hansen et al., 2012) and an increase in the occurrence frequencies of unusually warm seasonal and annual mean temperatures has been attributed in part to human influence (Stott et al., 2011; Christidis et al., 2012a, 2012b).

A large amount of evidence supports changes in daily data based temperature extreme indices consistent with warming, despite different data sets or different methods for data processing having been used (Section 2.6). The effects of human influence on daily temperature extremes is suggested by both qualitative and quantitative comparisons between observed and CMIP3 based modelled values of warm days and warm nights (the number of days exceeding the 90th percentile of daily maximum and daily minimum temperatures referred to as TX90p and TN90p, see also Section 2.7) and cold days and cold nights (the number of days with daily maximum and daily minimum temperatures below the 10th percentile referred to as TX10p and TN10p; see also Section 2.7). Trends in temperature extreme indices computed for Australia (Alexander and Arblaster, 2009) and the USA (Meehl et al., 2007a) using observations and simulations of the 20th century with nine GCMs that include both anthropogenic and natural forcings are found to be consistent. Both observations and model simulations show a decrease in the number of frost days, and an increase in the growing season length, heatwave duration and TN90p in the second half of the 20th century. Two of the models (PCM and CCSM3) with simulations that include only anthropogenic or only natural forcings 
indicate that the observed changes are simulated with anthropogenic forcings, but not with natural forcings (even though there are some differences in the details of the forcings). Morak et al. (2011) found that over many sub-continental regions, the number of warm nights (TN90p) shows detectable changes over the second half of the 20th century that are consistent with model simulated changes in response to historical external forcings. They also found detectable changes in indices of temperature extremes when the data were analysed over the globe as a whole. As much of the long-term change in TN90p can be predicted based on the interannual correlation of TN90p with mean temperature, Morak et al. (2013) conclude that the detectable changes are attributed in a multi-step approach (see Section 10.2.4) in part to GHG increases. Morak et al. (2013) have extended this analysis to TX10p, TN10p, TX90p as well as TN90p, using fingerprints from HadGEM1 and find detectable changes on global scales and in many regions (Figure 10.17).

Human influence has also been detected in two different measures of the intensity of extreme daily temperatures in a year. Zwiers et al. (2011) compared four extreme temperature variables including warmest daily maximum and minimum temperatures (annual maximum daily maximum and minimum temperatures, referred to as TXx, TNx) and coldest daily maximum and minimum temperatures (annual minimum daily maximum and minimum temperatures, referred to as $T X n, T N n)$ from observations and from simulations with anthropogenic forcing or anthropogenic and natural external forcings from seven GCMs. They consider these extreme daily temperatures to follow generalized extreme value (GEV) distributions with location, shape and scale parameters. They fit GEV distributions to the observed extreme temperatures with location parameters as linear functions of signals obtained from the model simulation. They found that both anthropogenic influence and combined influence of anthropogenic and natural forcing can be detected in all four extreme temperature variables at the global scale over the land, and also regionally over many large land areas (Figure 10.17). In a complementary study, Christidis et al. (2011) used an optimal fingerprint method to compare observed and modelled time-varying location parameters of extreme temperature distributions. They detected the effects of anthropogenic forcing on warmest daily temperatures in a single fingerprint analysis, and were able to separate the effects of natural from anthropogenic forcings in a two fingerprint analysis.
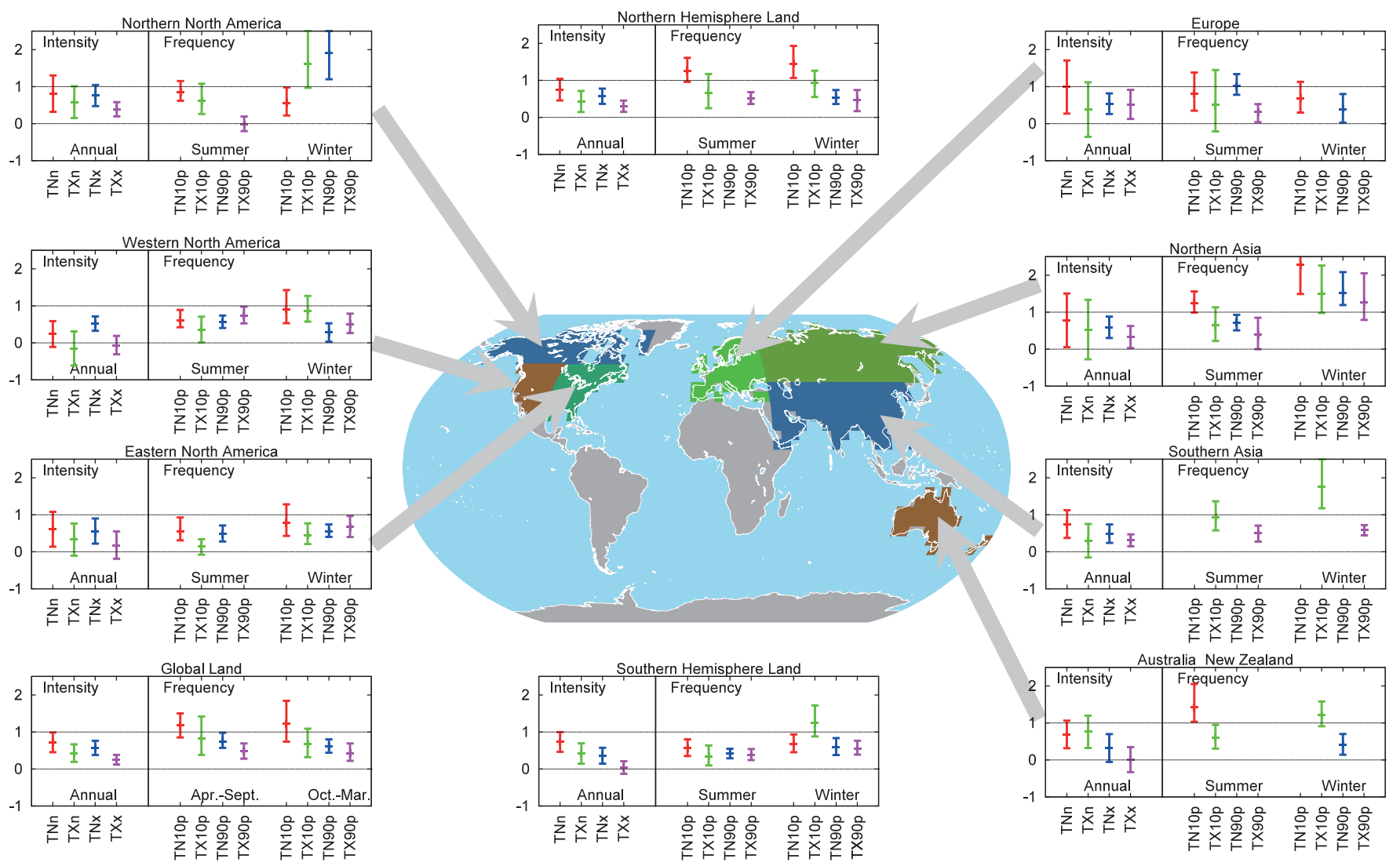

Figure 10.17 Detection results for changes in intensity and frequency of extreme events. The left side of each panel shows scaling factors and their $90 \%$ confidence intervals for intensity of annual extreme temperatures in response to external forcings for the period 1951-2000. TNn and TXn represent coldest daily minimum and maximum temperatures, respectively, while TNx and TXx represent warmest daily minimum and maximum temperatures (updated from Zwiers et al., 2011). Fingerprints are based on simulations of climate models with both anthropogenic and natural forcings. Right-hand sides of each panel show scaling factors and their $90 \%$ confidence intervals for changes in the frequency of temperature extremes for winter (October to March for the Northern Hemisphere and April to September for the Southern Hemisphere), and summer half years. TN10p, TX10p are respectively the frequency of cold nights and days (daily minimum and daily maximum temperatures falling below their 10th percentiles for the base period 1961-1990). TN90p and TX90p are the frequency of warm nights and days (daily minimum and daily maximum temperatures above their respective 90th percentiles calculated for the 1961-1990 base period (Morak et al., 2013) with fingerprints based on simulations of Hadley Centre Global Environmental Model 1 (HadGEM1) with both anthropogenic and natural forcings. Detection is claimed at the $5 \%$ significance level if the $90 \%$ confidence interval of a scaling factor is entirely above the zero line. Grey represents regions with insufficient data. 
Human influence on annual extremes of daily temperatures may be detected separately from natural forcing at the global scale (Christidis et al., 2011) and also at continental and sub-continental scales (Min et al., 2013). Over China, Wen et al. (2013) showed that anthropogenic influence may be separately detected from that of natural forcing in daily extreme temperatures (TNn, TNx, TXn and TXx), although the influence of natural forcing is not detected, and they also showed that the influence of GHGs in these indices may be separately detected from other anthropogenic forcings. Christidis et al. (2013) found that on a quasi-global scale, the cooling effect due to the decrease in tree cover and increase in grass cover since pre-industrial times as simulated by one ESM is detectable in the observed change of warm extremes. Urbanization may have also affected extreme temperatures in some regions; for example Zhou and Ren (2011) found that extreme temperature warms more in rural stations than in urban sites in China. The effect of land use change and urban heat Island is found to be small in GMST (Section 2.4.1.3). Consequently, this effect on extreme temperature is also expected to be small in the global average.

These new studies show that there is stronger evidence for anthropogenic forcing on changes in extreme temperatures than at the time of the SREX assessment. New evidence since SREX includes the separation of the influence of anthropogenic forcings from that of natural forcings on extreme daily temperatures at the global scale and to some extent at continental and sub-continental scales in some regions. These new results suggest more clearly the role of anthropogenic forcing on temperature extremes compared to results at the time of the SREX assessment. We assess that it is very likely that human influence has contributed to the observed changes in the frequency and intensity of daily temperature extremes on the global scale since the mid-20th century.

\subsubsection{Precipitation Extremes}

Observations have showed a general increase in heavy precipitation at the global scale. This appears to be consistent with the expected response to anthropogenic forcing as a result of an enhanced moisture content in the atmosphere but a direct cause-and-effect relationship between changes in external forcing and extreme precipitation had not been established at the time of the AR4. As a result, the AR4 concluded that increases in heavy precipitation were more likely than not consistent with anthropogenic influence during the latter half of the 20th century (Hegerl et al., 2007b).

Extreme precipitation is expected to increase with warming. A combination of evidence leads to this conclusion though by how much remains uncertain and may vary with time scale (Section 7.6.5). Observations and model projected future changes both indicate increase in extreme precipitation associated with warming. Analysis of observed annual maximum 1-day precipitation (RX1day) over global land areas with sufficient data smaples indicates a significant increase in extreme percipitation globally, with a median increase about $7 \%{ }^{\circ} \mathrm{C}^{-1} \mathrm{GMST}$ increase (Westra et al., 2013). CMIP3 and CMIP5 simulations project an increase in the globally averaged 20-year return values of annual maximum 24-hour precipitation amounts of about 6 to $7 \%$ with each degree Celsius of global mean warming, with the bulk of models simulating values in the range of 4 to $10 \%{ }^{\circ} \mathrm{C}^{-1}$ (Kharin et al., 2007; Kharin et al., 2013). Anthropogenic influence has been detected on various aspects of the global hydrological cycle (Stott et al., 2010), which is directly relevant to extreme precipitation changes. An anthropogenic influence on increasing atmospheric moisture content has been detected (see Section 10.3.2). A higher moisture content in the atmosphere would be expected to lead to stronger extreme precipitation as extreme precipitation typically scales with total column moisture if circulation does not change. An observational analysis shows that winter maximum daily precipitation in North America has statistically significant positive correlations with local atmospheric moisture (Wang and Zhang, 2008).

There is only a modest body of direct evidence that natural or anthropogenic forcing has affected global mean precipitation (see Section 10.3.2 and Figure 10.10), despite a robust expectation of increased precipitation (Balan Sarojini et al., 2012 ) and precipitation extremes (see Section 7.6.5). However, mean precipitation is expected to increase less than extreme precipitation because of energy constraints (e.g., Allen and Ingram, 2002). A perfect model analysis with an ensemble of GCM simulations shows that anthropogenic influence should be detectable in precipitation extremes in the second half of the 20th century at global and hemispheric scales, and at continental scale as well but less robustly (Min et al., 2008c), see also Hegerl et al. (2004). One study has also linked the observed intensification of precipitation extremes (including RX1day and annual maximum 5-day precipitation (RX5day)) over NH land areas to human influence using a limited set of climate models and observations (Min et al., 2011). However, the detection was less robust if using the fingerprint for combined anthropogenic and natural influences compared to that for anthropogenic influences only, possibly due to a number of factors including weak $\mathrm{S} / \mathrm{N}$ ratio and uncertainties in observation and model simulations. Also, models still have difficulties in simulating extreme daily precipitation directly comparable with those observed at the station level, which has been addressed to some extent by Min et al. (2011) by independently transforming annual precipitation extremes in models and observations onto a dimensionless scale that may be more comparable between the two. Detection of anthropogenic influence on smaller spatial scales is more difficult due to the increased level of noise and uncertainties and confounding factors on local scales. Fowler and Wilby (2010) suggested that there may have only been a $50 \%$ likelihood of detecting anthropogenic influence on UK extreme precipitation in winter at that time, and a very small likelihood of detecting it in other seasons.

Given the evidence of anthropogenic influence on various aspects of the global hydrological cycle that implies that extreme precipitation would be expected to have increased and some limited direct evidence of anthropogenic influence on extreme precipitation, but given also the difficulties in simulating extreme precipitation by climate models and limited observational coverage, we assess, consistent with SREX (Seneviratne et al., 2012) that there is medium confidence that anthropogenic forcing has contributed to a global scale intensification of heavy precipitation over the second half of the 20th century in land regions where observational coverage is sufficient for assessment.

\subsubsection{Drought}

AR4 concluded that that an increased risk of drought was more likely than not due to anthropogenic forcing during the second half of the 
20th century. This assessment was based on one detection study that identified an anthropogenic fingerprint in a global Palmer Drought Severity Index (PDSI) data set (Burke et al., 2006) and studies of some regions which indicated that droughts in those regions were linked to SST changes or to a circulation response to anthropogenic forcing. SREX (Seneviratne et al., 2012) assessed that there was medium confidence that anthropogenic influence has contributed to some changes in the drought patterns observed in the second half of the 20th century based on attributed impact of anthropogenic forcing on precipitation and temperature changes, and that there was low confidence in the assessment of changes in drought at the level of single regions.

Drought is a complex phenomenon that is affected by precipitation predominantly, as well as by other climate variables including temperature, wind speed and solar radiation (e.g., Seneviratne, 2012; Sheffield et al., 2012). It is also affected by non-atmospheric conditions such as antecedent soil moisture and land surface conditions. Trends in two important drought-related climate variables (precipitation and temperature) are consistent with the expected responses to anthropogenic forcing over the globe. However, there is large uncertainty in observed changes in drought (Section 2.6.2.3) and its attribution to causes globally. The evidence for changes in soil moisture indices and drought indices over the period since 1950 globally is conflicting (Hoerling et al., 2012; Sheffield et al., 2012; Dai, 2013), possibly due to the examination of different time periods, different forcing fields used to drive land surface models and uncertainties in land surface models (Pitman et al., 2009; Seneviratne et al., 2010; Sheffield et al., 2012). In a recent study, Sheffield et al. (2012) identify the representation of potential evaporation as solely dependent on temperature (using the Thornthwaite-based formulation) as a possible explanation for their finding that PDSI-based estimates might overestimate historical drought trends. This stands in partial contradiction to previous assessments suggesting that using a more sophisticated formulation (Penman-Monteith) for potential evaporation did not affect the results of respective PDSI trends (Dai, 2011; van der Schrier et al., 2011). Sheffield et al. (2012) argue that issues with the treatment of spurious trends in atmospheric forcing data sets and/or the choice of calibration periods explain these conflicting results. These conflicting results point out the challenges in quantitatively defining and detecting long-term changes in a multivariable phenomenon such as drought.

Recent long-term droughts in western North America cannot definitively be shown to lie outside the very large envelope of natural precipitation variability in this region (Cayan et al., 2010; Seager et al., 2010), particularly given new evidence of the history of high-magnitude natural drought and pluvial episodes suggested by palaeoclimatic reconstructions (see Chapter 5). Low-frequency tropical ocean temperature anomalies in all ocean basins appear to force circulation changes that promote regional drought (Hoerling and Kumar, 2003; Seager et al., 2005; Dai, 2011). Uniform increases in SST are not particularly effective in this regard (Schubert et al., 2009; Hoerling et al., 2012). Therefore, the reliable separation of natural variability and forced climate change will require simulations that accurately reproduce changes in largescale SST gradients at all time scales.

In summary, assessment of new observational evidence, in conjunction with updated simulations of natural and forced climate varia- bility, indicates that the AR4 conclusions regarding global increasing trends in droughts since the 1970s should be tempered. There is not enough evidence to support medium or high confidence of attribution of increasing trends to anthropogenic forcings as a result of observational uncertainties and variable results from region to region (Section 2.6.2.3). Combined with difficulties described above in distinguishing decadal scale variability in drought from long-term climate change we conclude consistent with SREX that there is low confidence in detection and attribution of changes in drought over global land areas since the mid-20th century.

\subsubsection{Extratropical Cyclones}

AR4 concluded that an anthropogenic influence on extratropical cyclones was not formally detected, owing to large internal variability and problems due to changes in observing systems. Although there is evidence that there has been a poleward shift in the storm tracks (see Section 2.6.4), various causal factors have been cited including oceanic heating (Butler et al., 2010) and changes in large-scale circulation due to effects of external forcings (Section 10.3.3). Increases in mid-latitude SST gradients generally lead to stronger storm tracks that are shifted poleward and increases in subtropical SST gradients may lead to storm tracks shifting towards the equator (Brayshaw et al., 2008; Semmler et al., 2008; Kodama and Iwasaki, 2009; Graff and LaCasce, 2012). However, changes in storm-track intensity are much more complicated, as they are sensitive to the competing effects of changes in temperature gradients and static stability at different levels and are thus not linked to GMST in a simple way (Ulbrich et al., 2009; O'Gorman, 2010). Overall global average cyclone activity is expected to change little under moderate GHG forcing ( $0^{\prime}$ Gorman and Schneider, 2008; Ulbrich et al., 2009; Bengtsson and Hodges, 2011), although in one study, human influence has been detected in geostrophic wind energy and ocean wave heights derived from sea level pressure data (Wang et al., 2009b).

\subsubsection{Tropical Cyclones}

AR4 concluded that 'anthropogenic factors more likely than not have contributed to an increase in tropical cyclone intensity' (Hegerl et al., 2007b). Evidence that supports this assessment was the strong correlation between the Power Dissipation Index (PDI, an index of the destructiveness of tropical cyclones) and tropical Atlantic SSTs (Emanuel, 2005; Elsner, 2006) and the association between Atlantic warming and the increase in GMST (Mann and Emanuel, 2006; Trenberth and Shea, 2006). Observations suggest an increase globally in the intensities of the strongest tropical cyclones (Elsner et al., 2008) but it is difficult to attribute such changes to particular causes (Knutson et al., 2010). The US Climate Change Science Program (CCSP; Kunkel et al., 2008) discussed human contributions to recent hurricane activity based on a two-step attribution approach. They concluded merely that it is very likely (Knutson et al., 2010) that human-induced increase in GHGs has contributed to the increase in SSTs in the hurricane formation regions and that over the past 50 years there has been a strong statistical connection between tropical Atlantic SSTs and Atlantic hurricane activity as measured by the PDI. Knutson et al. (2010), assessed that ' $\ldots$.. it remains uncertain whether past changes in tropical cyclone activity have exceeded the variability expected from natural causes.' Senevi- 
ratne et al. (2012) concurred with this finding. Section 14.6.1 gives a detailed account of past and future changes in tropical cyclones. This section assesses causes of observed changes.

Studies that directly attribute tropical cyclone activity changes to anthropogenic GHG emission are lacking. Among many factors that may affect tropical cyclone activity, tropical SSTs have increased and this increase has been attributed at least in part to anthropogenic forcing (Gillett et al., 2008a). However, there are diverse views on the connection between tropical cyclone activity and SST (see Section 14.6.1 for details). Strong correlation between the PDI and tropical Atlantic SSTs (Emanuel, 2005; Elsner, 2006) would suggest an anthropogenic influence on tropical cyclone activity. However, recent studies also suggest that regional potential intensity correlates with the difference between regional SSTs and spatially averaged SSTs in the tropics (Vecchi and Soden, 2007; Xie et al., 2010; Ramsay and Sobel, 2011) and projections are uncertain on whether the relative SST will increase over the 21 st century under GHG forcing (Vecchi et al., 2008; Xie et al., 2010; Villarini and Vecchi, 2012, 2013). Analyses of CMIP5 simulations suggest that while PDI over the North Atlantic is projected to increase towards late 21 st century no detectable change in PDI should be present in the 20th century (Villarini and Vecchi, 2013) . On the other hand, Emanuel et al. (2013) point out that while GCM hindcasts indeed predict little change over the 20th century, downscaling driving by reanalysis data that incorporate historical observations are in much better accord with observations and do indicate a late 20th century increase.

Some recent studies suggest that the reduction in the aerosol forcing (both anthropogenic and natural) over the Atlantic since the 1970s may have contributed to the increase in tropical cyclone activity in the region (see Section 14.6.1 for details), and similarly that aerosols may have acted to reduce tropical cyclone activity in the Atlantic in earlier years when aerosol forcing was increasing (Villarini and Vecchi, 2013). However, there are different views on the relative contribution of aerosols and decadal natural variability of the climate system to the observed changes in Atlantic tropical cyclone activity among these studies. Some studies indicate that aerosol changes have been the main driver (Mann and Emanuel, 2006; Evan et al., 2009; Booth et al., 2012; Villarini and Vecchi, 2012, 2013). Other studies infer the influence of natural variability to be as large as or larger than that from aerosols (Zhang and Delworth, 2009; Villarini and Vecchi, 2012, 2013).

Globally, there is low confidence in any long-term increases in tropical cyclone activity (Section 2.6.3) and we assess that there is low confidence in attributing global changes to any particular cause. In the North Atlantic region there is medium confidence that a reduction in aerosol forcing over the North Atlantic has contributed at least in part to the observed increase in tropical cyclone activity since the 1970 s. There remains substantial disagreement on the relative importance of internal variability, GHG forcing and aerosols for this observed trend. It remains uncertain whether past changes in tropical cyclone activity are outside the range of natural internal variability.

\subsubsection{Attribution of Weather and Climate Events}

Since many of the impacts of climate change are likely to manifest themselves through extreme weather, there is increasing interest in quantifying the role of human and other external influences on climate in specific weather events. This presents particular challenges for both science and the communication of results. It has so far been attempted for a relatively small number of specific events (e.g., Stott et al., 2004; Pall et al., 2011) although Peterson et al. (2012) attempt, for the first time, a coordinated assessment to place different high-impact weather events of the previous year in a climate perspective. In this assessment, selected studies are used to illustrate the essential principles of event attribution: see Stott et al. (2013) for a more exhaustive review.

Two distinct ways have emerged of framing the question of how an external climate driver like increased GHG levels may have contributed to an observed weather event. First, the 'attributable risk' approach considers the event as a whole, and asks how the external driver may have increased or decreased the probability of occurrence of an event of comparable magnitude. Second, the 'attributable magnitude' approach considers how different external factors contributed to the event or, more specifically, how the external driver may have increased the magnitude of an event of comparable occurrence probability. Hoerling et al. (2013) uses both methods to infer changes in magnitude and likelihood of the 2011 Texas heat wave.

Quantifying the absolute risk or probability of an extreme weather event in the absence of human influence on climate is particularly challenging. Many of the most extreme events occur because a self-reinforcing process that occurs only under extreme conditions amplifies an initial anomaly (e.g., Fischer et al., 2007). Hence the probability of occurrence of such events cannot, in general, be estimated simply by extrapolating from the distribution of less extreme events that are sampled in the historical record. Proxy records of pre-industrial climate generally do not resolve high-frequency weather, so inferring changes in probabilities requires a combination of hard-to-test distributional assumptions and extreme value theory. Quantifying absolute probabilities with climate models is also difficult because of known biases in their simulation of extreme events. Hence, with only a couple of exceptions (e.g., Hansen et al., 2012), studies have focussed on how risks have changed or how different factors have contributed to an observed event, rather than claiming that the absolute probability of occurrence of that event would have been extremely low in the absence of human influence on climate.

Even without considering absolute probabilities, there remain considerable uncertainties in quantifying changes in probabilities. The assessment of such changes will depend on the selected indicator, time period and spatial scale on which the event is analysed, and the way in which the event-attribution question is framed can substantially affect apparent conclusions. If an event occurs in the tail of the distribution, then a small shift in the distribution as a whole can result in a large increase in the probability of an event of a given magnitude: hence it is possible for the same event to be both 'mostly natural' in terms of attributable magnitude (if the shift in the distribution due to human influence is small compared to the anomaly in the natural variability that was the primary cause) and 'mostly anthropogenic' in terms of 
a) Autumn runoff, England and Wales

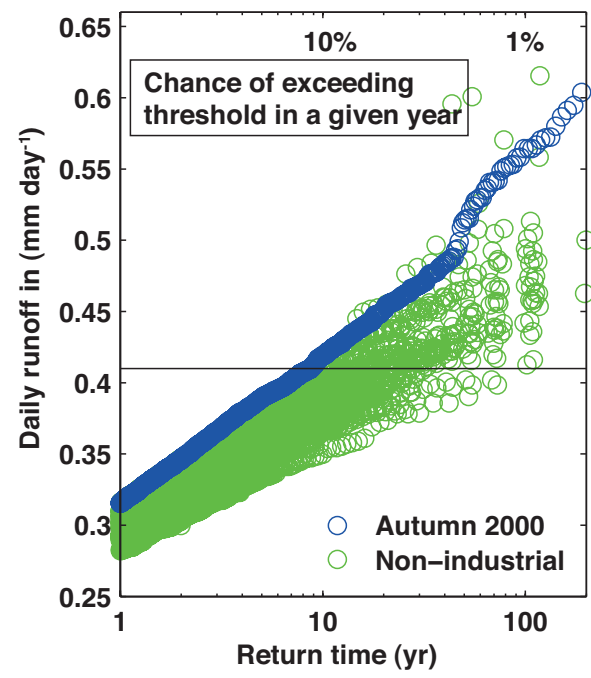

b) Spring flow, River Don, UK

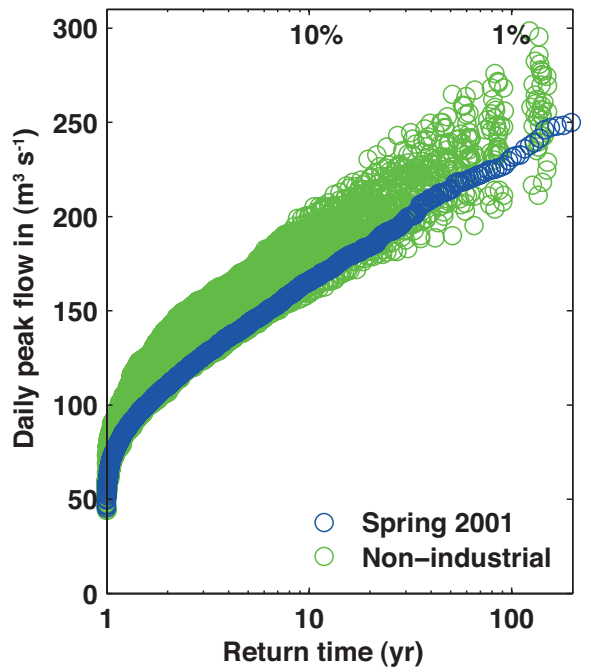

c) July temperatures, Western Russia

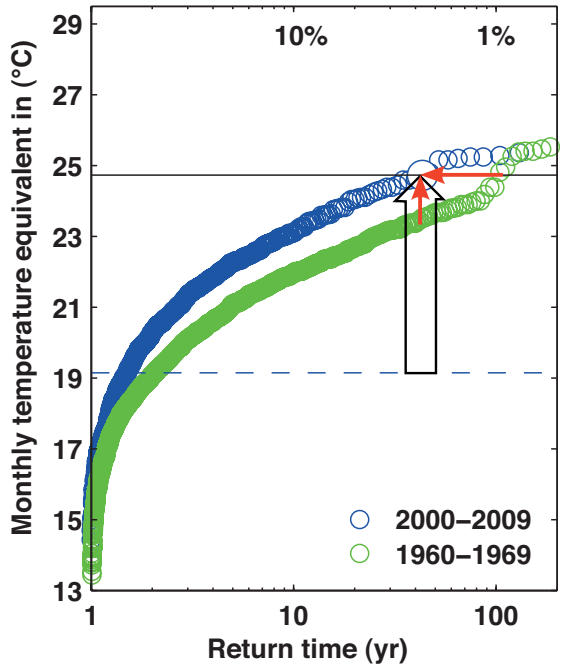

Figure 10.18| Return times for precipitation-induced floods aggregated over England and Wales for (a) conditions corresponding to September to November 2000 with boundary conditions as observed (blue) and under a range of simulations of the conditions that would have obtained in the absence of anthropogenic greenhouse warming over the 20th century (green) with different AOGCMs used to define the greenhouse signal, black horizontal line corresponds to the threshold exceeded in autumn 2000 (from Pall et al., 2011); (b) corresponding to January to March 2001 with boundary conditions as observed (blue) and under a range of simulations of the condition that would have obtained in the absence of anthropogenic greenhouse warming over the 20th century (green) adapted from Kay et al. (2011a); (c) return periods of temperature-geopotential height conditions in the model simulations for the 1960s (green) and the 2000s (blue). The vertical black arrow shows the anomaly of the 2010 Russian heat wave (black horizontal line) compared to the July mean temperatures of the 1960s (dashed line). The vertical red arrow gives the increase in temperature for the event whereas the horizontal red arrow shows the change in the return period (from Otto et al., 2012).

attributable risk (if human influence has increased its probability of occurrence by more than a factor of 2). These issues are discussed further using the example of the 2010 Russian heat wave below.

The majority of studies have focussed on quantifying attributable risk. Formally, risk is a function of both hazard and vulnerability (IPCC, 2012), although most studies attempting to quantify risk in the context of extreme weather do not explicitly use this definition, which is discussed further in Chapter 19 of WGII, but use the term as a shorthand for the probability of the occurrence of an event of a given magnitude. Any assessment of change in risk depends on an assumption of 'all other things being equal', including natural drivers of climate change and vulnerability. Given this assumption, the change in hazard is proportional to the change in risk, so we will follow the published literature and continue to refer to Fraction Attributable Risk, defined as FAR $=1-P 0 / P 1$, P0 being the probability of an event occurring in the absence of human influence on climate, and P1 the corresponding probability in a world in which human influence is included. FAR is thus the fraction of the risk that is attributable to human influence (or, potentially, any other external driver of climate change) and does not require knowledge of absolute values of $\mathrm{P} 0$ and $\mathrm{P} 1$, only their ratio.

For individual events with return times greater than the time scale over which the signal of human influence is emerging (30 to 50 years, meaning P0 and P1 less than 2 to $3 \%$ in any given year), it is impossible to observe a change in occurrence frequency directly because of the shortness of the observed record, so attribution is necessarily a multi-step procedure. Either a trend in occurrence frequency of more frequent events is attributed to human influence and a statistical model is then used to extrapolate to the implications for P0 and P1; or an attributable trend is identified in some other variable, such as surface temperature, and a physically based weather model is used to assess the implications for extreme weather risk. Neither approach is free of assumptions: no atmospheric model is perfect, but statistical extrapolation may also be misleading for reasons given above.

Pall et al. (2011) provide an example of multi-step assessment of attributable risk using a physically based model, applied to the floods that occurred in the UK in the autumn of 2000, the wettest autumn to have occurred in England and Wales since records began. To assess the contribution of the anthropogenic increase in GHGs to the risk of these floods, a several thousand member ensemble of atmospheric models with realistic atmospheric composition, SST and sea ice boundary conditions imposed was compared with a second ensemble with composition and surface temperatures and sea ice boundary conditions modified to simulate conditions that would have occurred had there been no anthropogenic increase in GHGs since 1900. Simulated daily precipitation from these two ensembles was fed into an empirical rainfall-runoff model and daily England and Wales runoff used as a proxy for flood risk. Results (Figure 10.18a) show that including the influence of anthropogenic greenhouse warming increases flood risk at the threshold relevant to autumn 2000 by around a factor of two in the majority of cases, but with a broad range of uncertainty: in $10 \%$ of cases the increase in risk is less than $20 \%$.

Kay et al. (2011a), analysing the same ensembles but using a more sophisticated hydrological model found a reduction in the risk of snow melt-induced flooding in the spring season (Figure 10.18b) which, aggregated over the entire year, largely compensated for the increased risk of precipitation-induced flooding in autumn. This illustrates an 
important general point: even if a particular flood event may have been made more likely by human influence on climate, there is no certainty that all kinds of flood events in that location, country or region have been made more likely.

Rahmstorf and Coumou (2011) provide an example of an empirical approach to the estimation of attributable risk applied to the 2010 Russian heat wave. They fit a nonlinear trend to central Russian temperatures and show that the warming that has occurred in this region since the 1960s has increased the risk of a heat wave of the magnitude observed in 2010 by around a factor of 5, corresponding to an FAR of 0.8 . They do not address what has caused the trend since 1960, although they note that other studies have attributed most of the large-scale warming over this period to the anthropogenic increase in GHG concentrations.

Dole et al. (2011) take a different approach to the 2010 Russian heat wave, focussing on attributable magnitude, analysing contributions from various external factors, and conclude that this event was 'mainly natural in origin'. First, observations show no evidence of a trend in occurrence frequency of hot Julys in western Russia, and despite the warming that has occurred since the 1960s, mean July temperatures in that region actually display a (statistically insignificant) cooling trend over the century as a whole, in contrast to the case for central and southern European summer temperatures (Stott et al., 2004). Members of the CMIP3 multi-model ensemble likewise show no evidence of a trend towards warming summers in central Russia. Second, Dole et al. (2011) note that the 2010 Russian event was associated with a strong blocking atmospheric flow anomaly, and even the complete 2010 boundary conditions are insufficient to increase the probability of a prolonged blocking event in this region, in contrast again to the situation in Europe in 2003. This anomaly in the large-scale atmospheric flow led to low-pressure systems being redirected around the blocking over Russia causing severe flooding in Pakistan which could so far not be attributed to anthropogenic causes (van Oldenborgh et al., 2012), highlighting that a global perspective is necessary to unravel the different factors influencing individual extreme events (Trenberth and Fasullo, 2012).

Otto et al. (2012) argue that it is possible to reconcile the results of Rahmstorf and Coumou (2011) with those of Dole et al. (2011) by relating the attributable risk and attributable magnitude approaches to framing the event attribution question. This is illustrated in Figure $10.18 c$, which shows return times of July temperatures in western Russia in a large ensemble of atmospheric model simulations for the 1960s (in green) and 2000s (in blue). The threshold exceeded in 2010 is shown by the solid horizontal line which is almost $6^{\circ} \mathrm{C}$ above $1960 \mathrm{~s}$ mean July temperatures, shown by the dashed line. The difference between the green and blue lines could be characterized as a $1.5^{\circ} \mathrm{C}$ increase in the magnitude of a 30-year event (the vertical red arrow, which is substantially smaller than the size of the anomaly itself, supporting the assertion that the event was 'mainly natural' in terms of attributable magnitude. Alternatively, it could be characterized as a threefold increase in the risk of the 2010 threshold being exceeded, supporting the assertion that risk of the event occurring was mainly attributable to the external trend, consistent with Rahmstorf and Coumou (2011). Rupp et al. (2012) and Hoerling et al. (2013) reach similar conclusions about the 2011 Texas heat wave, both noting the importance of La Niña conditions in the Pacific, with anthropogenic warming making a relatively small contribution to the magnitude of the event, but a more substantial contribution to the risk of temperatures exceeding a high threshold. This shows that the quantification of attributable risks and and changes in magnitude are affected by modelling error (e.g., Visser and Petersen, 2012) as they depend on the atmospheric model's ability to simulate the observed anomalies in the general circulation (Chapter 9).

Because much of the magnitude of these two heat waves is attributable to atmospheric flow anomalies, any evidence of a causal link between rising GHGs and the occurrence or persistence of flow anomalies such as blocking would have a very substantial impact on attribution claims. Pall et al. (2011) argue that, although flow anomalies played a substantial role in the autumn 2000 floods in the UK, thermodynamic mechanisms were primarily responsible for the change in risk between their ensembles. Regardless of whether the statistics of flow regimes themselves have changed, observed temperatures in recent years in Europe are distinctly warmer than would be expected for analogous atmospheric flow regimes in the past, affecting both warm and cold extremes (Yiou et al., 2007; Cattiaux et al., 2010).

In summary, increasing numbers of studies are finding that the probability of occurrence of events associated with extremely high temperatures has increased substantially due to the large-scale warming since the mid-20th century. Because most of this large-scale warming is very likely due to the increase in atmospheric GHG concentrations, it is possible to attribute, via a multi-step procedure, some of the increase in probability of these regional events to human influence on climate. Such an increase in probability is consistent with the implications of single-step attribution studies looking at the overall implications of increasing mean temperatures for the probabilities of exceeding temperature thresholds in some regions. We conclude that it is likely that human influence has substantially increased the probability of occurrence of heat waves in some locations. It is expected that attributable risks for extreme precipitation events are generally smaller and more uncertain, consistent with the findings in Kay et al. (2011a) and Pall et al. (2011). The science of event attribution is still confined to case studies, often using a single model, and typically focussing on high-impact events for which the issue of human influence has already arisen. While the increasing risk of heat waves measured as the occurrence of a previous temperature record being exceeded can simply be explained by natural variability superimposed by globally increasing temperature, conclusions for holistic events including general circulation patterns are specific to the events that have been considered so far and rely on the representation of relevant processes in the model.

Anthropogenic warming remains a relatively small contributor to the overall magnitude of any individual short-term event because its magnitude is small relative to natural random weather variability on short time scales (Dole et al., 2011; Hoerling et al., 2013). Because of this random variability, weather events continue to occur that have been made less likely by human influence on climate, such as extreme winter cold events (Massey et al., 2012), or whose probability of occurrence has not been significantly affected either way. Quantifying how different external factors contribute to current risks, and how risks are 
changing, is possible with much higher confidence than quantifying absolute risk. Biases in climate models, uncertainty in the probability distribution of the most extreme events and the ambiguity of paleoclimatic records for short-term events mean that it is not yet possible to quantify the absolute probability of occurrence of any observed weather event in a hypothetical pristine climate. At present, therefore, the evidence does not support the claim that we are observing weather events that would, individually, have been extremely unlikely in the absence of human-induced climate change, although observed trends in the concurrence of large numbers of events (see Section 10.6.1) may be more easily attributable to external factors. The most important development since AR4 is an emerging consensus that the role of external drivers of climate change in specific extreme weather events, including events that might have occurred in a pre-industrial climate, can be quantified using a probabilistic approach.

\subsection{Multi-century to Millennia Perspective}

Evaluating the causes of climate change before the 20th century is important to test and improve our understanding of the role of internal and forced natural climate variability for the recent past. This section draws on assessment of temperature reconstructions of climate change over the past millennium and their uncertainty in Chapter 5 (Table 5.A.1; Sections 5.3.5 and 5.5.1 for regional records), and on comparisons of models and data over the pre-instrumental period in Chapters 5 and 9 (Sections 5.3.5, 5.5.1 and 9.5.3), and focuses on the evidence for the contribution by radiatively forced climate change to reconstructions and early instrumental records. In addition, the residual variability that is not explained by forcing from palaeoclimatic records provides a useful comparison to estimates of climate model internal variability. The model dependence of estimates of internal variability is an important uncertainty in detection and attribution results.

The inputs for detection and attribution studies for periods covered by indirect, or proxy, data are affected by more uncertainty than those from the instrumental period (see Chapter 5), owing to the sparse data coverage, particularly further back in time, and uncertainty in the link between proxy data and, for example, temperature. Records of past radiative influences on climate are also uncertain (Section 5.2; see Schmidt et al., 2011; Schmidt et al., 2012). For the preindustrial part of the last millennium changes in solar, volcanic, GHG forcing, and land use change, along with a small orbital forcing are potentially important external drivers of climate change. Estimates of solar forcing (Figure 5.1a; Box 10.2) are uncertain, particularly in their amplitude, as well as in modelling, for example, of the influence of solar forcing on atmospheric circulation involving stratospheric dynamics (see Box 10.2; Gray et al., 2010). Estimates of past volcanism are reasonably well established in their timing, but the magnitude of the RF of individual eruptions is uncertain (Figure 5.1a). It is possible that large eruptions had a more moderated climate effect than simulated by many climate models due to faster fallout associated with larger particle size (Timmreck et al., 2009), or increased amounts of injected water vapour (Joshi and Jones, 2009). Reconstructed changes in land cover and its effect on climate are also uncertain (Kaplan et al., 2009; Pongratz et al., 2009). Forcing of WMGHGs shows only very subtle variations over the last millennium up to 1750 . This includes a small drop and partial recovery in the 17th century (Section 6.2.3, Figure 6.7), followed by increases in GHG concentrations with industrialization since the middle of the 18th century (middle of the 19th century for $\mathrm{N}_{2} \mathrm{O}$, Figure 6.11).

When interpreting reconstructions of past climate change with the help of climate models driven with estimates of past forcing, it helps that the uncertainties in reconstructions and forcing are independent from each other. Thus, uncertainties in forcing and reconstructions combined should lead to less, rather than more similarity between fingerprints of forced climate change and reconstructions, making it improbable that the response to external drivers is spuriously detected. However, this is the case only if all relevant forcings and their uncertainties are considered, reducing the risk of misattribution due to spurious correlations between external forcings, and if the data are homogeneous and statistical tests properly applied (e.g., Legras et al., 2010). Hence this section focuses on work that considers all relevant forcings simultaneously.

\subsubsection{Causes of Change in Large-Scale Temperature over the Past Millennium}

Despite the uncertainties in reconstructions of past NH mean temperatures, there are well-defined climatic episodes in the last millennium that can be robustly identified (Chapter 5 , see also Figure 10.19). Chapter 5 concludes that in response to solar, volcanic and anthropogenic $\mathrm{RFs}$, climate models simulate temperature changes in the $\mathrm{NH}$ which are generally consistent in magnitude and timing with reconstructions, within their broad uncertainty ranges (Section 5.3.5).

\subsubsection{Role of External Forcing in the Last Millennium}

The AR4 concluded that 'A substantial fraction of the reconstructed $\mathrm{NH}$ inter-decadal temperature variability of the seven centuries prior to 1950 is very likely attributable to natural external forcing'. The literature since the AR4, and the availability of more simulations of the last millennium with more complete forcing (see Schmidt et al., 2012), including solar, volcanic and GHG influences, and generally also land use change and orbital forcing) and more sophisticated models, to a much larger extent coupled climate or coupled ESMs (Chapter 9), some of them with interactive carbon cycle, strengthens these conclusions.

Most reconstructions show correlations with external forcing that are similar to those found between pre-Paleoclimate Modelling Intercomparison Project Phase 3 (PMIP3) simulations of the last millennium and forcing, suggesting an influence by external forcing (FernándezDonado et al., 2013). From a global scale average of new regional reconstructions, Past Global Changes 2k (PAGES 2k) Consortium (2013) find that periods with strong volcanic and solar forcing combined occurring over the last millennium show significantly cooler conditions than randomly selected periods from the last two millennia. Detection analyses based on PMIP3 and CMIP5 model simulations for the years from 850 to 1950 and also from 850 to 1850 find that the fingerprint of external forcing is detectable in all reconstructions of NH mean temperature considered (Schurer et al., 2013; see Figure 10.19), but only in about half the cases considered does detection also occur prior to 1400 . The authors find a smaller response to forcing in reconstructions than simulated, but this discrepancy is consistent with 
uncertainties in forcing or proxy response to it, particularly associated with volcanism. The discrepancy is reduced when using more strongly smoothed data or omitting major volcanic eruptions from the analysis. The level of agreement between fingerprints from multiple models in response to forcing and reconstructions decreases earlier in time, and the forced signal is detected only in about half the cases considered when analysing the period 851 to 1401 . This may be partly due to weaker forcing and larger forcing uncertainty early in the millennium and partly due to increased uncertainty in reconstructions. Detection results indicate a contribution by external drivers to the warm conditions in the 11th to 12th century, but cannot explain the warmth around the 10th century in some of the reconstructions (Figure 10.19). This detection of a role of external forcing extends work reported in AR4 back into to the 9th century CE.
Detection and attribution studies support results from modelling studies that infer a strong role of external forcing in the cooling of $\mathrm{NH}$ temperatures during the Little Ice Age (LIA; see Chapter 5 and Glossary). Both model simulations (Jungclaus et al., 2010) and results from detection and attribution studies (Hegerl et al., 2007a; Schurer et al., 2013) suggest that a small drop in GHG concentrations may have contributed to the cool conditions during the 16th and 17th centuries. Note, however, that centennial variations of GHG during the late Holocene are very small relative to their increases since pre-industrial times (Section 6.2.3). The role of solar forcing is less clear except for decreased agreement if using very large solar forcing (e.g., Ammann et al., 2007; Feulner, 2011). Palastanga et al. (2011) demonstrate that neither a slowdown of the thermohaline circulation nor a persistently negative NAO alone can explain the reconstructed temperature pattern over Europe during the periods $1675-1715$ and 1790-1820.

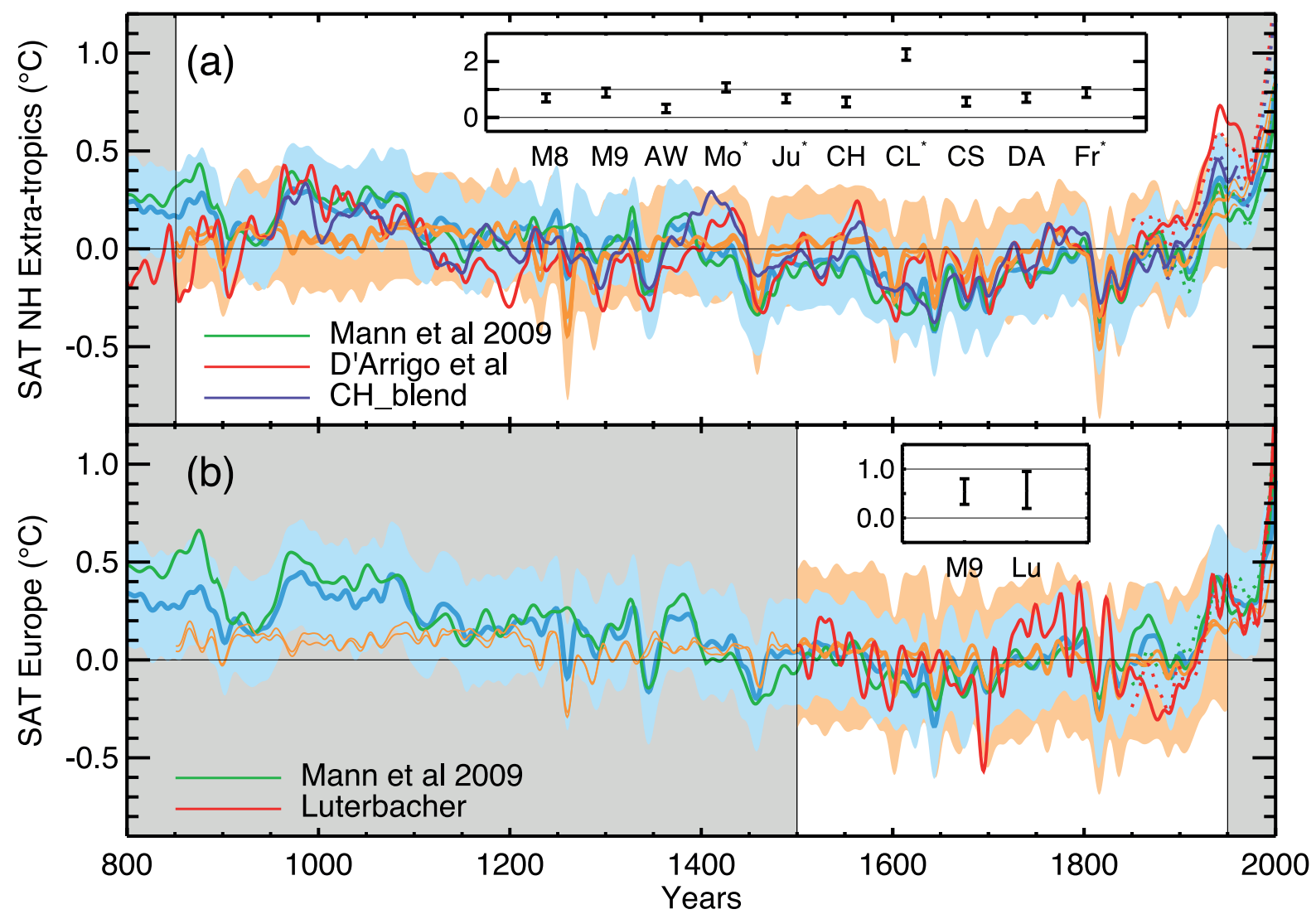

Figure 10.19 | The top panel compares the mean annual Northern Hemisphere (NH) surface air temperature from a multi-model ensemble to several NH temperature reconstructions. These reconstructions are: $\mathrm{CH}$-blend from Hegerl et al. (2007a) in purple, which is a reconstruction of $30^{\circ} \mathrm{N}$ to $90^{\circ} \mathrm{N}$ land only (Mann et al., 2009), plotted for the region $30^{\circ} \mathrm{N}$ to $90^{\circ} \mathrm{N}$ land and sea (green) and D'Arrigo et al. (2006) in red, which is a reconstruction of $20^{\circ} \mathrm{N}$ to $90^{\circ} \mathrm{N}$ land only. The dotted coloured lines show the corresponding instrumental data. The multi-model mean for the reconstructed domain is scaled to fit each reconstruction in turn, using a total least squares (TLS) method. The best estimate of the detected forced signal is shown in orange (as an individual line for each reconstruction; lines overlap closely) with light orange shading indicating the range expected if accounting for internal variability. The best fit scaling values for each reconstruction are given in the insert as well as the detection results for six other reconstructions (M8; M9 (Mann et al., 2008 , 2009); AW (Ammann and Wahl, 2007); Mo (Moberg et al., 2005); Ju (Juckes et al., 2007); CH (Hegerl et al., 2007a); CL (Christiansen and Ljungqvist, 2011) and inverse regressed onto the instrumental record CS; DA (D'Arrigo et al., 2006); Fr (Frank et al., 2007). An asterisk next to the reconstruction name indicates that the residuals (over the more robustly reconstructed period 1401-1950) are inconsistent with the internal variability generated by the combined control simulations of all climate models investigated (for details see Schurer et al., 2013). The ensemble average of a data-assimilation simulation (Goosse et al., 2012b) is plotted in blue, for the region $30^{\circ} \mathrm{N}$ to $90^{\circ} \mathrm{N}$ land and sea, with the error range shown in light blue shading. The bottom panel is similar to the top panel, but showing the European region, following Hegerl et al. (2011a) but using the simulations and method in Schurer et al. (2013). The detection analysis is performed for the period 1500-1950 for two reconstructions: Luterbacher et al. (2004)(representing the region $35^{\circ} \mathrm{N}$ to $70^{\circ} \mathrm{N}, 25^{\circ} \mathrm{W}$ to $40^{\circ} \mathrm{E}$, "land only, labelled 'Lu' in the insert") shown in red, and Mann et al. (2009) (averaged over the region $25^{\circ} \mathrm{N}$ to $65^{\circ} \mathrm{N}, 0^{\circ}$ to $60^{\circ} \mathrm{E}$, land and sea, labelled 'M9' in the insert), shown in green. As in the top panel, best fit estimates are shown in dark orange with uncertainty range due to internal variability shown in light orange. The data assimilation from Goosse et al. (2012a), constrained by the Mann et al. (2009) reconstruction is shown in blue, with error range in light blue. All data are shown with respect to the mean of the period covered by the white part of the figure (850-1950 for the NH, 1500-1950 for European mean data). 
Data assimilation studies support the conclusion that external forcing, together with internal climate variability, provides a consistent explanation of climate change over the last millennium. Goosse et al. (2010, 2012a, 2012b) select, from a very large ensemble with an EMIC, the individual simulations that are closest to the spatial reconstructions of temperature between $30^{\circ} \mathrm{N}$ and $60^{\circ} \mathrm{N}$ by Mann et al. (2009) accounting for reconstruction uncertainties. The method also varies the external forcing within uncertainties, determining a combined realization of the forced response and internal variability that best matches the data. Results (Figure 10.19) show that simulations reproduce the target reconstruction within the uncertainty range, increasing confidence in the consistency of the reconstruction and the forcing. The results suggest that long-term circulation anomalies may help to explain the hemispheric warmth early in the millennium, although results vary dependent on input parameters of the method.

\subsubsection{Role of Individual Forcings}

Volcanic forcing shows a detectable influence on large-scale temperature (see AR4; Chapter 5), and volcanic forcing plays an important role in explaining past cool episodes, for example, in the late 17th and early 19th centuries (see Chapter 5 and 9; Hegerl et al., 2007b; Jungclaus et al., 2010; Miller et al., 2012). Schurer et al. (2013) separately detect the response to GHG variations between 1400 and 1900 in most NH reconstructions considered, and that of solar and volcanic forcing combined in all reconstructions considered.

Even the multi-century perspective makes it difficult to distinguish century-scale variations in $\mathrm{NH}$ temperature due to solar forcing alone from the response to other forcings, due to the few degrees of freedom constraining this forcing (see Box 10.2). Hegerl et al. (2003, 2007a) found solar forcing detectable in some cases. Simulations with higher than best guess solar forcing may reproduce the warm period around 1000 more closely, but the peak warming occurs about a century earlier in reconstructions than in solar forcing and with it model simulations (Jungclaus et al., 2010; Figure 5.8; Fernández-Donado et al., 2013). Even if solar forcing were on the high end of estimates for the last millennium, it would not be able to explain the recent warming according both to model simulations (Ammann et al., 2007; Tett et al., 2007; Feulner, 2011) and detection and attribution approaches that scale the temporal fingerprint of solar forcing to best match the data (Hegerl et al., 2007a; Schurer et al., 2013; Figure 10.19). Some studies suggest that particularly for millennial and multi-millenial time scales orbital forcing may be important globally (Marcott et al., 2013) and for high-latitude trends (Kaufman et al., 2009) based on a comparison of the correspondence between long-term Arctic cooling in models and data though the last millennium up to about 1750 (see also PAGES 2k Consortium, 2013).

\subsubsection{Estimates of Internal Climate Variability}

The interdecadal and longer-term variability in large-scale temperatures in climate model simulations with and without past external forcing is quite different (Tett et al., 2007; Jungclaus et al., 2010), consistent with the finding that a large fraction of temperature variance in the last millennium has been externally driven. The residual variability in past climate that is not explained by changes in RF provides an estimate of internal variability for $\mathrm{NH}$ mean temperature that is not directly derived from climate model simulation. This residual variability is somewhat larger than control simulation variability for some reconstructions if the comparison is extended to the full period since 850 CE (Schurer et al., 2013), However, when extracting 50- and 60-year trends from this residual variability, the distribution of these trends is similar to the multi-model control simulation ensemble used in Schurer et al. (2013). In all cases considered, the most recent 50-and 60-year trend from instrumental data is far outside the range of any 50-year trend in residuals from reconstructions of $\mathrm{NH}$ mean temperature of the past millennium.

\subsubsection{Changes of Past Regional Temperature}

Several reconstructions of European regional temperature variability are available (Section 5.5). While Bengtsson et al. (2006) emphasized the role of internal variability in pre-industrial European climate as reconstructed by Luterbacher et al. (2004), Hegerl et al. (2011a) find a detectable response to external forcing in summer temperatures in the period 1500-1900, for winter temperatures during 1500-1950 and 1500-2000; and throughout the record for spring. The fingerprint of the forced response shows coherent time evolution between models and reconstructed temperatures over the entire analysed period (compare to annual results in Figure 10.19, using a larger multi-model ensemble). This suggests that the cold European winter conditions in the late 17th and early 19th century and the warming in between were at least partly externally driven.

Data assimilation results focussing on the European sector suggests that the explanation of forced response combined with internal variability is self-consistent (Goosse et al., 2012a, Figure 10.19). The assimilated simulations reproduce the warmth of the MCA better than the forced only simulations do. The response to individual forcings is difficult to distinguish from each other in noisier regional reconstructions. An epoch analysis of years immediately following strong, largely tropical, volcanic eruptions shows that European summers show detectable fingerprints of volcanic response, while winters show a noisy response of warming in northern Europe and cooling in southern Europe (Hegerl et al., 2011a). Landrum et al. (2013) suggest similar volcanic responses for North America, with warming in the north of the continent and cooling in the south. There is also evidence for a decrease in SSTs following tropical volcanic forcing in tropical reconstructions over the past 450 years (D'Arrigo et al., 2009). There is also substantial literature suggesting solar influences on regional climate reconstructions, possibly due to circulation changes, for example, changes in Northern Annular Modes (e.g., Kobashi et al., 2013; see Box 10.2).

\subsubsection{Summary: Lessons from the Past}

Detection and attribution studies strengthen results from AR4 that external forcing contributed to past climate variability and change prior to the 20th century. Ocean-Atmosphere General Circulation Models (OAGCMs) simulate similar changes on hemispheric and annual scales as those by simpler models used earlier, and enable detection of regional and seasonal changes. Results suggest that volcanic forcing and GHG forcing in particular are important for explaining past changes in NH temperatures. Results from data assimilation runs confirm 
that the combination of internal variability and external forcing provides a consistent explanation of the last millennium and suggest that changes in circulation may have further contributed to climate anomalies. The role of external forcing extends to regional records, for example, European seasonal temperatures. In summary, it is very unlikely that NH temperature variations from 1400 to 1850 can be explained by internal variability alone. There is medium confidence that external forcing contributed to NH temperature variability from 850 to 1400 . There is medium confidence that external forcing (anthropogenic and natural forcings together) contributed to European temperatures of the last five centuries.

\subsection{Implications for Climate System Properties and Projections}

Detection and Attribution results can be used to constrain predictions of future climate change (see Chapters 11 and 12) and key climate system properties. These properties include: the Equilibrium Climate Sensitivity (ECS), which determines the long-term equilibrium warming response to stable atmospheric composition, but not accounting for vegetation or ice sheet changes (Section 12.5.3; see Box 12.2); the transient climate response (TCR), which is a measure of the magnitude of transient warming while the climate system, particularly the deep ocean, is not in equilibrium; and the transient climate response to cumulative $\mathrm{CO}_{2}$ emissions (TCRE), which is a measure of the transient warming response to a given mass of $\mathrm{CO}_{2}$ injected into the atmosphere, and combines information on both the carbon cycle and climate response. TCR is more tightly constrained by the observations of transient warming than ECS. The observational constraints on TCR, ECS and TCRE assessed here focus on information provided by recent observed climate change, complementing analysis of feedbacks and climate modelling information, which are assessed in Chapter 9. The assessment in this chapter also incorporates observational constraints based on palaeoclimatic information, building on Chapter 5 , and contributes to the overall synthesis assessment in Chapter 12 (Box 12.2).

Because neither ECS nor TCR is directly observed, any inference about them requires some form of climate model, ranging in complexity from a simple zero-dimensional energy balance box model to OAGCMs (Hegerl and Zwiers, 2011). Constraints on estimates of long-term climate change and equilibrium climate change from recent warming hinge on the rate at which the ocean has taken up heat (Section 3.2), and by the extent to which recent warming has been reduced by cooling from aerosol forcing. Therefore, attempts to estimate climate sensitivity (transient or equilibrium) often also estimate the total aerosol forcing and the rate of ocean heat uptake, which are discussed in Section 10.8.3. The AR4 contained a detailed discussion on estimating quantities relevant for projections, and included an appendix with the relevant estimation methods. Here, we build on this assessment, repeating information and discussion only where necessary to provide context.

\subsubsection{Transient Climate Response}

The AR4 discussed for the first time estimates of the TCR. TCR was originally defined as the warming at the time of $\mathrm{CO}_{2}$ doubling (i.e., after 70 years) in a $1 \% \mathrm{yr}^{-1}$ increasing $\mathrm{CO}_{2}$ experiment (see Hegerl et al., 2007b), but like ECS, it can also be thought of as a generic property of the climate system that determines the global temperature response $\Delta T$ to any gradual increase in $\mathrm{RF}, \Delta F$, taking place over an approximately 70 -year time scale, normalized by the ratio of the forcing change to the forcing due to doubling $\mathrm{CO}_{2}, F_{2 \times \mathrm{CO} 2}: \mathrm{TCR}=F_{2 \times \mathrm{CO} 2} \Delta T / \Delta F$ (Frame et al., 2006; Gregory and Forster, 2008; Held et al., 2010; Otto et al., 2013). This generic definition of the TCR has also been called the 'Transient Climate Sensitivity' (Held et al., 2010). TCR is related to ECS and the global energy budget as follows: $\mathrm{ECS}=F_{2 \times \mathrm{CO}_{2}} / \alpha$, where $\alpha$ is the sensitivity parameter representing the net increase in energy flux to space per degree of warming given all feedbacks operating on these time scales. Hence, by conservation of energy, $\mathrm{ECS}=F_{2 \times \operatorname{co2}} \Delta T /(\Delta F-\Delta Q)$, where $\Delta Q$ is the change in the rate of increase of climate system heat content in response to the forcing $\Delta F$. On these time scales, deep ocean heat exchange affects the surface temperature response as if it were an enhanced radiative damping, introducing a slow, or 'recalcitrant', component of the response which would not be reversed for many decades even if it were possible to return RF to pre-industrial values (Held et al., 2010): hence the difficulty of placing an upper bound on ECS from observed surface warming alone (Forest et al., 2002; Frame et al., 2006). Because $\Delta Q$ is always positive at the end of a period of increasing forcing, before the climate system has re-equilibrated, TCR is always less than ECS, and since $\Delta Q$ is uncertain, TCR is generally better constrained by observations of recent climate change than ECS.

Because TCR focuses on the short- and medium-term response, constraining TCR with observations is a key step in narrowing estimates of future global temperature change in the relatively short term and under scenarios where forcing continues to increase or peaks and declines (Frame et al., 2006). After stabilization, the ECS eventually becomes the relevant climate system property. Based on observational constraints alone, the AR4 concluded that TCR is very likely to be larger than $1^{\circ} \mathrm{C}$ and very unlikely to be greater than $3.5^{\circ} \mathrm{C}$ (Hegerl et al., 2007b). This supported the overall assessment that the transient climate response is very unlikely greater than $3^{\circ} \mathrm{C}$ and very likely greater than $1^{\circ} \mathrm{C}$ (Meehl et al., 2007a). New estimates of the TCR are now available.

Scaling factors derived from detection and attribution studies (see Section 10.2) express how model responses to GHGs and aerosols need to be scaled to match the observations over the historical period. These scaled responses were used in AR4 to provide probabilistic projections of both TCR and future changes in global temperature in response to these forcings under various scenarios (Allen et al., 2000; Stott and Kettleborough, 2002; Stott et al., 2006, 2008b; Kettleborough et al., 2007; Meehl et al., 2007b; Stott and Forest, 2007). Allen et al. (2000), Frame et al. (2006) and Kettleborough et al. (2007) demonstrate a near linear relationship between 20th century warming, TCR and warming by the mid-21st century as parameters are varied in Energy Balance Models, justifying this approach. Forster et al. (2013) show how the ratio $\Delta T$ I $\Delta F$ does depend on the forcing history, with very rapid increases in forcing giving lower values: hence any inference from past attributable warming to future warming or TCR depends on a model (which may be simple or complex, but ideally physically based) to relate these quantities. Such inferences also depend on forcing estimates and projections. Recent revisions to RF (see Chapter 8) suggest higher net anthropogenic forcing over the 20th century, and hence a smaller estimated 
TCR. Stott et al. (2008b) demonstrated that optimal detection analysis of 20th century temperature changes (using HadCM3) are able to exclude the very high and low temperature responses to aerosol forcing. Consequently, projected 21st century warming may be more closely constrained than if the full range of aerosol forcings is used (Andreae et al., 2005). Stott and Forest (2007) demonstrate that projections obtained from such an approach are similar to those obtained by constraining EMIC parameters from observations. Stott et al. (2011), using HadGEM2-ES, and Gillett et al. (2012), using CanESM2, both show that the inclusion of observations between 2000 and 2010 in such an analysis reduces the uncertainties in projected warming in the 21st century, and tends to constrain the maximum projected warming to below that projected using data to 2000 only (Stott et al, 2006). Such an improvement is consistent with prior expectations of how additional data will narrow uncertainties (Stott and Kettleborough, 2002).

TCR estimates have been derived using a variety of methods (Figure 10.20a). Knutti and Tomassini (2008) compare EMIC simulations with 20th century surface and ocean temperatures to derive a probability density function for TCR skewed slightly towards lower values with a 5 to $95 \%$ range of $1.1^{\circ} \mathrm{C}$ to $2.3^{\circ} \mathrm{C}$. Libardoni and Forest (2011) take a similar approach with a different EMIC and include atmospheric data and, under a variety of assumptions, obtain 5 to $95 \%$ ranges for TCR spanning $0.9^{\circ} \mathrm{C}$ to $2.4^{\circ} \mathrm{C}$. Updating this study to include data to 2004 gives results that are essentially unchanged. Using a single model and observations from 1851 to 2010 Gillett et al. (2012) derive a 5 to $95 \%$ range of $1.3^{\circ} \mathrm{C}$ to $1.8^{\circ} \mathrm{C}$ and using a single model, but using multiple sets of observations and analysis periods ending in 2010 and beginning in 1910 or earlier, Stott et al. (2011) derive 5 to $95 \%$ ranges that were generally between $1^{\circ} \mathrm{C}$ and $3^{\circ} \mathrm{C}$. Both Stott et al. (2011) and Gillett et al. (2012) find that the inclusion of data between 2000 and 2010 helps to constrain the upper bound of TCR. Gillett et al. (2012) find that the inclusion of data prior to 1900 also helps to constrain TCR, though Stott et al. (2011) do not. Gillett et al. (2013) account for a broader range of model and observational uncertainties, in particular addressing the efficacy of non- $\mathrm{CO}_{2}$ gases, and find a range of $0.9^{\circ} \mathrm{C}$ to $2.3^{\circ} \mathrm{C}$. Several of the estimates of TCR that were cited by Hegerl et al. (2007b) may have underestimated non- $\mathrm{CO}_{2}$ efficacies relative to the more recent estimates in Forster et al. (2007). Because observationally constrained estimates of TCR are based on the ratio between past attributable warming and past forcing, this could account for a high bias in some of the inputs used for the AR4 TCR estimate.

Held et al. (2010) show that a two-box model originally proposed by Gregory (2000), distinguishing the 'fast' and 'recalcitrant' responses, fits both historical simulations and instantaneous doubled $\mathrm{CO}_{2}$ simulations of the GFDL coupled model CM2.1. The fast response has a relaxation time of 3 to 5 years, and the historical simulation is almost completely described by this fast component of warming. Padilla et al. (2011) use this simple model to derive an observationally constrained estimate of the TCR of $1.3^{\circ} \mathrm{C}$ to $2.6^{\circ} \mathrm{C}$. Schwartz (2012) uses this twotime scale formulation to obtain TCR estimates ranging from $0.9^{\circ} \mathrm{C}$ to $1.9^{\circ} \mathrm{C}$, the lower values arising from higher estimates of forcing over the 20th century. Otto et al. (2013) update the analysis of Gregory et al. (2002) and Gregory and Forster (2008) using forcing estimates from Forster et al. (2013) to obtain a 5 to $95 \%$ range for TCR of $0.9^{\circ} \mathrm{C}$ to $2.0^{\circ} \mathrm{C}$ comparing the decade $2000-2009$ with the period 1860-1879.
They note, however, the danger of overinterpreting a single, possibly anomalous, decade, and report a larger TCR range of $0.7^{\circ} \mathrm{C}$ to $2.5^{\circ} \mathrm{C}$ replacing the 2000s with the 40 years 1970-2009.

Tung et al. (2008) examine the response to the 11-year solar cycle using discriminant analysis, and find a high range for TCR: $>2.5^{\circ} \mathrm{C}$ to $3.6^{\circ} \mathrm{C}$ However, this estimate may be affected by different mechanisms by which solar forcing affects climate (see Box 10.2). The authors attempt to minimize possible aliasing with the response to other forcings in the 20th century and with internal climate variability, although some influence by them cannot be ruled out.

Rogelj et al. (2012) take a somewhat different approach, using a simple climate model to match the distribution of TCR to observational constraints and a consensus distribution of ECS (which will itself have been informed by recent climate change), following Meinshausen et al. (2009). Harris et al. (2013) estimate a distribution for TCR based on a large sample of emulated GCM equilibrium responses, constrained by multiannual mean observations of recent climate and adjusted to account for additional uncertainty associated with model structural deficiencies (Sexton et al., 2012). The equilibrium responses are scaled by global temperature changes associated with the sampled model variants, reweighting the projections based on the likelihood that they correctly replicate observed historical changes in surface temperature, to predict the TCR distribution. Both of these studies represent a combination of multiple lines of evidence, although still strongly informed by recent observed climate change, and hence are assessed here for completeness.

Based on this evidence, including the new 21st century observations that were not yet available to AR4, we conclude that, on the basis of constraints provided by recent observed climate change, TCR is likely to lie in the range $1{ }^{\circ} \mathrm{C}$ to $2.5^{\circ} \mathrm{C}$ and extremely unlikely to be greater than $3^{\circ} \mathrm{C}$. This range for TCR is smaller than given at the time of AR4, due to the stronger observational constraints and the wider range of studies now available. Our greater confidence in excluding high values of TCR arises primarily from higher and more confident estimates of past forcing: estimates of TCR are not strongly dependent on observations of ocean heat uptake.

\subsubsection{Constraints on Long-Term Climate Change and the Equilibrium Climate Sensitivity}

The equilibrium climate sensitivity (ECS) is defined as the warming in response to a sustained doubling of carbon dioxide in the atmosphere relative to pre-industrial levels (see AR4). The equilibrium to which the ECS refers to is generally assumed to be an equilibrium involving the ocean-atmosphere system, which does not include Earth system feedbacks such as long-term melting of ice sheets and ice caps, dust forcing or vegetation changes (see Chapter 5 and Section 12.5.3). The ECS cannot be directly deduced from transient warming attributable to GHGs, or from TCR, as the role of ocean heat uptake has to be taken into account (see Forest et al., 2000; Frame et al., 2005; Knutti and Hegerl, 2008). Estimating the ECS generally relies on the paradigm of a comparison of observed change with results from a physically based climate model, sometimes a very simple one, given uncertainty in the model, data, RF and due to internal variability. 
For example, estimates can be based on the simple box model introduced in Section 10.8.1, ECS $=F_{2 \times \mathrm{CO} 2} \Delta T /(\Delta F-\Delta Q)$. Simple energy balance calculations rely on a very limited representation of climate response time scales, and cannot account for nonlinearities in the climate system that may lead to changes in feedbacks for different forcings (see Chapter 9). Alternative approaches are estimates that use climate model ensembles with varying parameters that evaluate the ECS of individual models and then infer the probability density function (PDF) for the ECS from the model-data agreement or by using optimization methods (Tanaka et al., 2009).

As discussed in the AR4, the probabilistic estimates available in the literature for climate system parameters, such as ECS and TCR have all been based, implicitly or explicitly, on adopting a Bayesian approach and therefore, even if it is not explicitly stated, involve using some kind of prior information. The shape of the prior has been derived from expert judgement in some studies, observational or experimental evidence in others or from the distribution of the sample of models available. In all cases the constraint by data, for example, from transient warming, or observations related to feedbacks is fairly weak on the upper tail of ECS (e.g., Frame et al., 2005). Therefore, results are sensitive to the prior assumptions (Tomassini et al., 2007; Knutti and Hegerl, 2008; Sanso and Forest, 2009; Aldrin et al., 2012). When the prior distribution fails to taper off for high sensitivities, as is the case for uniform priors (Frame et al., 2005), this leads to long tails (Frame et al., 2005; Annan and Hargreaves, 2011; Lewis, 2013). Uniform priors have been criticized (e.g., Annan and Hargreaves, 2011; Pueyo, 2012; Lewis, 2013) since results assuming a uniform prior in ECS translates instead into a strongly structured prior on climate feedback parameter and vice versa (Frame et al., 2005; Pueyo, 2012). Objective Bayesian analyses attempt to avoid this paradox by using a prior distribution that is invariant to parameter transforms and rescaling, for example, a Jeffreys prior (Lewis, 2013). Estimated probability densities based on priors that are strongly non-uniform in the vicinity of the best fit to the data, as is typically the case for the Jeffreys prior in this instance, can peak at values very different from the location of the best fit, and hence need to be interpreted carefully. To what extent results are sensitive to priors can be evaluated by using different priors, and this has been done more consistently in studies than at the time of AR4 (see Figure 10.20b) and is assessed where available, illustrated in Figure 10.20. Results will also be sensitive to the extent to which uncertainties in forcing (Tanaka et al., 2009), models and observations and internal climate variability are taken into account, and can be acutely sensitive to relatively arbitrary choices of observation period, choice of truncation in estimated covariance matrices and so forth (Lewis, 2013), illustrating the importance of sensitivity studies. Analyses that make a more complete effort to estimate all uncertainties affecting the model-data comparison lead to more trustworthy results, but end up with larger uncertainties (Knutti and Hegerl, 2008).

The detection and attribution chapter in AR4 (Hegerl et al., 2007b) concluded that 'Estimates based on observational constraints indicate that it is very likely that the equilibrium climate sensitivity is larger than $1.5^{\circ} \mathrm{C}$ with a most likely value between $2^{\circ} \mathrm{C}$ and $3^{\circ} \mathrm{C}$. The following sections discuss evidence since AR4 from several lines of evidence, followed by an overall assessment of ECS based on observed climate changes, and a subset of available new estimates is shown in Figure 10.20b.

\subsubsection{Estimates from Recent Temperature Change}

As estimates of ECS based on recent temperature change can only sample atmospheric feedbacks that occur with presently evolving climate change, they provide information on the 'effective climate sensitivity' (e.g., Forest et al., 2008). As discussed in AR4, analyses based on global scale data find that within data uncertainties, a strong aerosol forcing or a large ocean heat uptake might have masked a strong greenhouse warming (see, e.g., Forest et al., 2002; Frame et al., 2005; Stern, 2006; Roe and Baker, 2007; Hannart et al., 2009; Urban and Keller, 2009; Church et al., 2011). This is consistent with the finding that a set of models with a large range of ECS and aerosol forcing could be consistent with the observed warming (Kiehl, 2007). Consequently, such analyses find that constraints on aerosol forcing are essential to provide tighter constraints on future warming (Tanaka et al., 2009; Schwartz et al., 2010). Aldrin et al. (2012) analyse the observed record from 1850 to 2007 for hemispheric means of surface temperature, and upper $700 \mathrm{~m}$ ocean heat content since 1955. The authors use a simple climate model and a Markov Chain Monte Carlo Bayesian technique for analysis. The authors find a quite narrow range of ECS, which narrows further if using a uniform prior in 1/ECS rather than ECS (Figure 10.20). If observations are updated to 2010 and forcing estimates including further indirect aerosol effects are used (following Skeie et al., 2011), this yields a reduced upper tail (see Figure 10.20b, dash dotted). However, this estimate involves a rather simple model for internal variability, hence may underestimate uncertainties. Olson et al. (2012) use similar global scale constraints and surface temperature to 2006 , and ocean data to 2003 and arrive at a wide range if using a uniform prior in ECS, and a quite well constrained range if using a prior derived from current mean climate and Last Glacial Maximum (LGM) constraints (see Figure 10.20b). Some of the differences between Olson et al. (2012) and Aldrin et al. (2012) may be due to structural differences in the model used (Aldrin et al. use a simple EBM while Olson use the UVIC EMIC), some due to different statistical methods and some due to use of global rather than hemispheric temperatures in the latter work. An approach based on regressing forcing histories used in 20th century simulations on observed surface temperatures (Schwartz, 2012) estimates ranges of ECS that encompass the AR4 ranges if accounting for data uncertainty (Figure 10.20). Otto et al. (2013) updated the Gregory et al. (2002) global energy balance analysis (see equation above), using temperature and ocean heat content data to 2009 and estimates of RF that are approximately consistent with estimates from Chapters 7 and 8 , and ocean heat uptake estimates that are consistent with Chapter 3 and find that inclusion of recent deep ocean heat uptake and temperature data considerably narrow estimates of ECS compared to results using data to the less recent past.

Estimates of ECS and TCR that make use of both spatial and temporal information, or separate the GHG attributable warming using fingerprint methods, can yield tighter estimates (e.g., Frame et al., 2005; Forest et al., 2008; Libardoni and Forest, 2011). The resulting GHG attributable warming tends to be reasonably robust to uncertainties in aerosol forcing (Section 10.3.1.1.3). Forest et al. (2008) have updated their earlier study using a newer version of the MIT model and five different surface temperature data sets (Libardoni and Forest, 2011). Correction of statistical errors in estimation procedure pointed out by Lewis (see Lewis, 2013) changes their result only slightly (Libardoni 
and Forest, 2013). The overarching 5 to $95 \%$ range of effective climate sensitivity widens to $1.2^{\circ} \mathrm{C}$ to $5.3^{\circ} \mathrm{C}$ when all five data sets are used, and constraints on effective ocean diffusivity become very weak (Forest et al., 2008). Uncertainties would likely further increase if estimates of forcing uncertainty, for example, due to natural forcings, are also included (Forest et al., 2006). Lewis (2013) reanalysed the data used in Forest et al. (2006) using an objective Bayesian method (see discussion at top of section). The author finds that use of a Jeffreys prior narrows the upper tail considerably, to $3.6^{\circ} \mathrm{C}$ for the 95 th percentile. When revising the method, omitting upper air data, and adding 6 more years of data a much reduced 5 to $95 \%$ range of $1.2{ }^{\circ} \mathrm{C}$ to $2.2^{\circ} \mathrm{C}$ results (see Figure 10.20), similar to estimates by Ring et al. (2012) using data to 2008 . Lewis's upper limit extends to $3.0^{\circ} \mathrm{C}$ if accounting for forcing and surface temperature uncertainty (Lewis, 2013). Lewis (2013) also reports a range of $1.1^{\circ} \mathrm{C}$ to $2.9^{\circ} \mathrm{C}$ using his revised diagnostics and the Forest et al. (2006) statistical method, whereas adding 9 more years to the Libardoni and Forest (2013) corrected diagnostic (after Libardoni and Forest, 2011; Figure 10.20; using an expert prior in both cases), does not change results much (Figure 10.20b). The differences between results reported in Forest et al. (2008); Libardoni and Forest (2011); Lewis (2013); Libardoni and Forest (2013) are still not fully understood, but appear to be due to a combination of sensitivity of results to the choice of analysis period as well as differences in diagnostics and statistical approach.

In summary, analyses that use the most recent decade find a tightening of the range of ECS based on a combination of recent heat uptake and surface temperature data. Results consistently give low probability to ECS values under $1.0^{\circ} \mathrm{C}$ (Figure 10.20). The mode of the PDFs varies considerably with period considered as expected from the influence of internal variability on the single realization of observed climate change. Estimates including the most recent data tend to have reduced upper tails (Libardoni and Forest, 2011; Aldrin et al., 2012 and update; Ring et al., 2012 and update cf. Figure 10.20; Lewis, 2013; Otto et al., 2013), although further uncertainty in statistical assumptions and structural uncertainties in simple models used, as well as neglected uncertainties, for example, in forcings, increase assessed uncertainty.

\subsubsection{Estimates Based on Top of the Atmosphere Radiative Balance}

With the satellite era, measurements are now long enough to allow direct estimates of variations in the energy budget of the planet, although the measurements are not sufficiently accurate to determine absolute top of the atmosphere (TOA) fluxes or trends (see Section 2.3 and Box 13.1). Using a simple energy balance relationship between net energy flow towards the Earth, net forcing and a climate feedback parameter and the satellite measurements Murphy et al. (2009) made direct estimates of the climate feedback parameter as the regression coefficient of radiative response against GMST. The feedback parameter in turn is inversely proportional to the ECS (see above, also Forster and Gregory, 2006). Such regression based estimates are, however, subject to uncertainties (see Section 7.2.5.7; see also, Gregory and Forster, 2008; Murphy and Forster, 2010). Lindzen and Choi (2009) used data from the radiative budget and simple energy balance models over the tropics to investigate feedbacks in climate models. Their result suggests that climate models overestimate the outgoing shortwave radiation compared to Earth Radiation Budget Experiment (ERBE) data, but this result was found unreliable owing to use of a limited sample of periods and of a domain limited to low latitudes (Murphy and Forster, 2010). Lindzen and Choi (2011) address some of these criticisms (Chung et al., 2010; Trenberth et al., 2010), but the results remains uncertain. For example, the lag-lead relationship between TOA balance and SST (Lindzen and Choi, 2011) is replicated by Atmospheric Model Intercomparison Project (AMIP) simulations where SST cannot respond (Dessler, 2011). Hence, as discussed in Section 7.2.5.7, the influence of internal temperature variations on short time scales seriously affects such estimates of feedbacks. In addition, the energy budget changes that are used to derive feedbacks are also affected by RF, which Lindzen and Choi (2009) do not account for. Murphy and Forster (2010) further question if estimates of the feedback parameter are suitable to estimate the ECS, as multiple time scales are involved in feedbacks that contribute to climate sensitivity (Knutti and Hegerl, 2008; Dessler, 2010). Lin et al. (2010a) use data over the 20th century combined with an estimate of present TOA imbalance based on modelling (Hansen et al., 2005a) to estimate the energy budget of the planet and give a best estimate of ECS of $3.1^{\circ} \mathrm{C}$, but do not attempt to estimate a distribution that accounts fully for uncertainties. In conclusion, measurement and methodological uncertainties in estimates of the feedback parameter and the ECS from short-term variations in the satellite period preclude strong constraints on ECS. When accounting for these uncertainties, estimates of ECS based on the TOA radiation budget appear consistent with those from other lines of evidence within large uncertainties (e.g., Forster and Gregory, 2006; Figure 10.20b).

\subsubsection{Estimates Based on Response to Volcanic Forcing or Internal Variability}

Some analyses used in AR4 were based on the well observed forcing and responses to major volcanic eruptions during the 20th century. The constraint is fairly weak because the peak response to short-term volcanic forcing depends nonlinearly on ECS (Wigley et al., 2005; Boer et al., 2007). Recently, Bender et al. (2010) re-evaluated the constraint and found a close relationship in 9 out of 10 AR4 models between the shortwave TOA imbalance, the simulated response to the eruption of Mt Pinatubo and the ECS. Applying the constraint from observations suggests a range of ECS of $1.7^{\circ} \mathrm{C}$ to $4.1^{\circ} \mathrm{C}$. This range for ECS is subject to observational uncertainty and uncertainty due to internal climate variability, and is derived from a limited sample of models. Schwartz (2007) tried to relate the ECS to the strength of natural variability using the fluctuation dissipation theorem but studies suggest that the observations are too short to support a well constrained and reliable estimate and would yield an underestimate of sensitivity (Kirk-Davidoff, 2009); and that assuming single time scales is too simplistic for the climate system (Knutti and Hegerl, 2008) . Thus, credible estimates of ECS from the response to natural and internal variability do not disagree with other estimates, but at present cannot provide more reliable estimates of ECS.

\subsubsection{Paleoclimatic Evidence}

Palaeoclimatic evidence is promising for estimating ECS (Edwards et al., 2007). This section reports on probabilistic estimates of ECS derived from paleoclimatic data by drawing on Chapter 5 information 
on forcing and temperature changes. For periods of past climate, which were close to radiative balance or when climate was changing slowly, for example, the LGM, radiative imbalance and with it ocean heat uptake is less important than for the present (Sections 5.3.3.1 and 5.3.3.2). Treating the RF due to ice sheets, dust and $\mathrm{CO}_{2}$ as forcings rather than feedbacks implies that the corresponding RF contributions are associated with considerable uncertainties (see Section 5.2.2.3). Koehler et al. (2010) used an estimate of LGM cooling along with its uncertainties together with estimates of LGM RF and its uncertainty to derive an overall estimate of climate sensitivity. This method accounts for the effect of changes in feedbacks for this very different climatic state using published estimates of changes in feedback factors (see Section 5.3.3.2; Hargreaves et al., 2007; Otto-Bliesner et al., 2009). The authors find a best estimate of $2.4^{\circ} \mathrm{C}$ and a 5 to $95 \%$ range of ECS from $1.4^{\circ} \mathrm{C}$ to $5.2^{\circ} \mathrm{C}$, with sensitivities beyond $6^{\circ} \mathrm{C}$ difficult to reconcile with the data. In contrast, Chylek and Lohmann (2008b) estimate the ECS to be $1.3^{\circ} \mathrm{C}$ to $2.3^{\circ} \mathrm{C}$ based on data for the transition from the LGM to the Holocene. However, the true uncertainties are likely larger due to uncertainties in relating local proxies to large-scale temperature change observed over a limited time (Ganopolski and von Deimling, 2008; Hargreaves and Annan, 2009). The authors also use an aerosol RF estimate that may be high (see response by Chylek and Lohmann, 2008a; Ganopolski and von Deimling, 2008).

At the time of the AR4, several studies were assessed in which parameters in climate models had been perturbed systematically in order to estimate ECS, and further studies have been published since, some making use of expanded data for LGM climate change (see Section 5.3.3.2, Table 5.3). Sometimes substantial differences between estimates based on similar data reflect not only differences in assumptions on forcing and use of data, but also structural model uncertainties, for example, in how feedbacks change between different climatic states (e.g., Schneider von Deimling et al., 2006; Hargreaves et al., 2007; (see also Otto-Bliesner et al., 2009). Holden et al. (2010) analysed which versions of the EMIC Genie are consistent with LGM tropical SSTs and find a $90 \%$ range of $2.0^{\circ} \mathrm{C}$ to $5.0^{\circ} \mathrm{C}$. Recently, new data synthesis products have become available for assessment with climate model simulations of the LGM which together with further data cover much more of the LGM ocean and land areas, although there are still substantial gaps and substantial data uncertainty (Section 5.3.3). An analysis of the recent SST and land temperature reconstructions for the LGM compared to simulations with an EMIC suggests a $90 \%$ range of $1.4^{\circ} \mathrm{C}$ to $2.8^{\circ} \mathrm{C}$ for ECS, with SST data providing a narrower range and lower values than land data only (see Figure 10.20; Schmittner et al., 2011). However, structural model uncertainty as well as data uncertainty may increase this range substantially (Fyke and Eby, 2012; Schmittner et al., 2012). Hargreaves et al. (2012) derived a relationship between ECS and LGM response for seven model simulations from PMIP2 simulations and found a linear relationship between tropical cooling and ECS (see Section 5.3.3.2) which has been used to derive an estimate of ECS (Figure 10.20); and has been updated using PMIP3 simulations (Section 5.3.3.2). However, uncertainties remain as the relationship is dependent on the ensemble of models used.

Estimates of ECS from other, more distant paleoclimate periods (e.g., Royer et al., 2007; Royer, 2008; Pagani et al., 2009; Lunt et al., 2010) are difficult to directly compare, as climatic conditions were very different from today and as climate sensitivity can be state dependent, as discussed above. Also, the response on very long time scales is determined by the Earth System Sensitivity, which includes very slow feedbacks by ice sheets and vegetation (see Section 12.5.3). Paleosens Members (2012) reanalysed the relationship between RF and temperature response from paleoclimatic studies, considering Earth system feedbacks as forcings in order to derive an estimate of ECS that is limited to atmospheric feecbacks (sometimes referred to as Charney sensitivity and directly comparable to ECS), and find that resulting estimates are reasonably consistent over the past 65 million years (see detailed discussion in Section 5.3.1). They estimate a $95 \%$ range of $1.1^{\circ} \mathrm{C}$ to $7.0^{\circ} \mathrm{C}$, largely based on the past 800,000 years. However, uncertainties in paleoclimate estimates of ECS are likely to be larger than from the instrumental record, for example, due to changes in feedbacks between different climatic states. In conclusion, estimates of ECS have continued to emerge from palaeoclimatic periods that indicate that $\mathrm{ECS}$ is very likely less than $6^{\circ} \mathrm{C}$ and very likely greater than $1.0^{\circ} \mathrm{C}$ (see Section 5.3.3).

\subsubsection{Combining Evidence and Overall Assessment}

Most studies find a lower $5 \%$ limit for ECS between $1{ }^{\circ} \mathrm{C}$ and $2^{\circ} \mathrm{C}$ (Figure 10.20). The combined evidence thus indicates that the net feedbacks to $\mathrm{RF}$ are significantly positive. At present, there is no credible individual line of evidence that yields very high or very low climate sensitivity as best estimate. Some recent studies suggest a low climate sensitivity (Chylek et al., 2007; Schwartz et al., 2007; Lindzen and Choi, 2009). However, these are based on problematic assumptions, for example, about the climate's response time, the cause of climate fluctuations, or neglect uncertainty in forcing, observations and internal variability (as discussed in Foster et al., 2008; Knutti and Hegerl, 2008; Murphy and Forster, 2010). In some cases the estimates of the ECS have been refuted by testing the method of estimation with a climate model of known sensitivity (e.g., Kirk-Davidoff, 2009).

Several authors (Annan and Hargreaves, 2006; Hegerl et al., 2006; Annan and Hargreaves, 2010) had proposed combining estimates of climate sensitivity from different lines of evidence by the time of AR4; these and recent work is shown in the panel 'combined' in Figure 10.20. Aldrin et al. (2012) combined the Hegerl et al. (2006) estimate based on the last millennium with their estimate based on the 20th century; and Olson et al. (2012) combined weak constraints from climatology and the LGM in their prior, updated by data on temperature changes. This approach is robust only if the lines of evidence used are truly independent. The latter is hard to evaluate when using prior distributions based on expert knowledge (e.g., Libardoni and Forest, 2011). If lines of evidence are not independent, overly confident assessments of equilibrium climate sensitivity may result (Henriksson et al., 2010; Annan and Hargreaves, 2011).

In conclusion, estimates of the Equilibrium Climate Sensitivity (ECS) based on multiple and partly independent lines of evidence from observed climate change, including estimates using longer records of surface temperature change and new palaeoclimatic evidence, indicate that there is high confidence that ECS is extremely unlikely less than $1^{\circ} \mathrm{C}$ and medium confidence that the ECS is likely between $1.5^{\circ} \mathrm{C}$ and $4.5^{\circ} \mathrm{C}$ and very unlikely greater than $6^{\circ} \mathrm{C}$. They complement the 

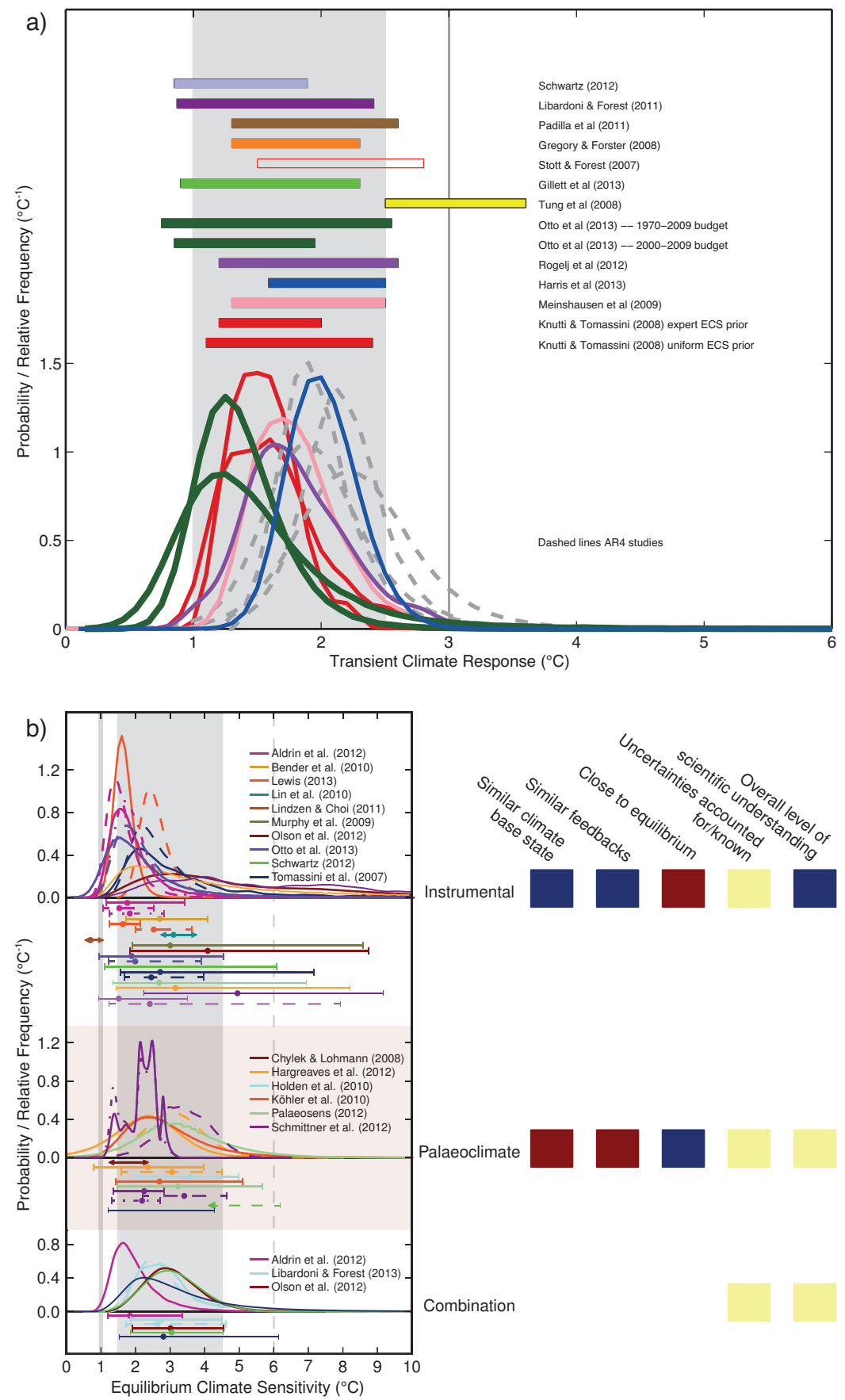

Figure 10.20 (a) Examples of distributions of the transient climate response (TCR, top) and the equilibrium climate sensitivity (ECS, bottom) estimated from observational constraints. Probability density functions (PDFs), and ranges (5 to $95 \%$ ) for the TCR estimated by different studies (see text). The grey shaded range marks the very likely range of $1{ }^{\circ} \mathrm{C}$ to $2.5^{\circ} \mathrm{C}$ for TCR and the grey solid line represents the extremely unlikely $<3^{\circ} \mathrm{C}$ upper bound as assessed in this section. Representative distributions from AR4 shown as dashed lines and open bar. (b) Estimates of ECS are compared to overall assessed likely range (solid grey), with solid line at $1{ }^{\circ} \mathrm{C}$ and a dashed line at $6^{\circ} \mathrm{C}$. The figure compares some selected old estimates used in AR4 (no labels, thin lines; for references see Supplementary Material) with new estimates available since AR4 (labelled, thicker lines). Distributions are shown where available, together with 5 to $95 \%$ ranges and median values (circles). Ranges that are assessed as being incomplete are marked by arrows; note that in contrast to the other estimates Schwartz (2012), shows a sampling range and Chylek and Lohmann a 95\% range. Estimates are based on changes over the instrumental period (top row); and changes from palaeoclimatic data (2nd row). Studies that combine multiple lines of evidence are shown in the bottom panel. The boxes on the right-hand side indicate limitations and strengths of each line of evidence, for example, if a period has a similar climatic base state, if feedbacks are similar to those operating under $\mathrm{CO}_{2}$ doubling, if the observed change is close to equilibrium, if, between all lines of evidence plotted, uncertainty is accounted for relatively completely, and summarizes the level of scientific understanding of this line of evidence overall. A blue box indicates an overall line of evidence that is well understood, has small uncertainty, or many studies and overall high confidence. Pale yellow indicates medium, and dark red low, confidence (i.e., poorly understood,very few studies, poor agreement, unknown limitations, after Knutti and Hegerl, 2008). Where available, results are shown using several different prior distributions; for example for Aldrin et al. (2012) solid shows the result using a uniform prior in ECS, which is shown as updated to 2010 in dash-dots; dashed: uniform prior in 1/ECS; and in bottom panel, result combining with Hegerl et al. (2006) prior, For Lewis (2013), dashed shows results using the Forest et al. (2006) diagnostic and an objective Bayesian prior, solid a revised diagnostic. For Otto et al. (2013), solid is an estimate using change to 1979-2009, dashed using the change to 2000-2009. Palaeoclimate: Hargreaves et al. (2012) is shown in solid, with dashed showing an update based on PMIP3 simulations (see Chapter 5); For Schmittner et al. (2011), solid is land-and-ocean, dashed land-only, and dash-dotted is ocean-only diagnostic. 
evaluation in Chapter 9 and support the overall assessment in Chapter 12 that concludes between all lines of evidence with high confidence that ECS is likely in the range $1.5^{\circ} \mathrm{C}$ to $4.5^{\circ} \mathrm{C}$. Earth system feedbacks can lead to different, probably larger, warming than indicated by ECS on very long time scales.

\subsubsection{Consequences for Aerosol Forcing and Ocean Heat Uptake}

Some estimates of ECS also yield estimates of aerosol forcing that are consistent with observational records, which we briefly mention here. Note that the estimate will reflect any forcings with a time or timespace pattern resembling aerosol forcing that is not explicitly included in the overall estimate (see discussion in Olson et al., 2012), for example, BC on snow; and should hence be interpreted as an estimate of aerosol plus neglected forcings. Estimates will also vary with the method applied and diagnostics used (e.g., analyses including spatial information will yield stronger results). Murphy et al. (2009) use correlations between surface temperature and outgoing shortwave and longwave flux over the satellite period to estimate how much of the total recent forcing has been reduced by aerosol total reflection, which they estimate as $-1.1 \pm 0.4 \mathrm{~W} \mathrm{~m}^{-2}$ from 1970 to 2000 ( 1 standard deviation), while Libardoni and Forest (2011), see also Forest et al. (2008), based on the 20th century, find somewhat lower estimates, namely a $90 \%$ bound of -0.83 to $-0.19 \mathrm{~W} \mathrm{~m}^{-2}$ for the 1980 s relative to preindustrial. Lewis (2013), using similar diagnostics but an objective Bayesian method, estimates a total aerosol forcing of about -0.6 to $-0.1 \mathrm{~W}$ $\mathrm{m}^{-2}$ or -0.6 to $0.0 \mathrm{~W} \mathrm{~m}^{-2}$ dependent on diagnostic used. The range of the aerosol forcing estimates that are based on the observed climate change are in-line with the expert judgement of the effective RF by aerosol radiation and aerosol cloud interactions combined (ERFaci+ari; Chapter 7) of $-0.9 \mathrm{~W} \mathrm{~m}^{-2}$ with a range from -1.9 to $-0.1 \mathrm{~W} \mathrm{~m}^{-2}$ that has been guided by climate models that include aerosol effects on mixed-phase and convective clouds in addition to liquid clouds, satellite studies and models that allow cloud-scale responses (see Section 7.5.2).

Several estimates of ECS also estimate a parameter that describe the efficiency with which the ocean takes up heat, e.g., effective global vertical ocean diffusivity (e.g., Tomassini et al., 2007; Forest et al., 2008; Olson et al., 2012; Lewis, 2013). Forest and Reynolds (2008) find that the effective global ocean diffusivity $K_{v}$ in many of the CMIP3 models lies above the median value based on observational constraints, resulting in a positive bias in their ocean heat uptake. Lewis (2013) similarly finds better agreement for small values of effective ocean diffusivity. However, such a finding was very sensitive to data sets used for surface temperature (Libardoni and Forest, 2011) and ocean data (Sokolov et al., 2010), is somewhat sensitive to the diagnostic applied (Lewis, 2013), and limited by difficulties observing heat uptake in the deep ocean (see, e.g., Chapters 3 and 13). Olson et al. (2012) and Tomassini et al. (2007) find that data over the historical period provide only a weak constraint on background ocean effective diffusivity. Comparison of the vertical profiles of temperature and of historical warming in models and observations suggests that the ocean heat uptake efficiency may be typically too large (Kuhlbrodt and Gregory, 2012; Section 13.4.1; see also Sections 9.4.2, 10.4.1, 10.4.3). If effective diffusivity were high in models this might lead to a tendency to bias ocean warming high relative to surface warming; but this uncertainty makes only a small contribution to uncertainty in TCR (Knutti and Tomassini, 2008; Kuhlbrodt and Gregory, 2012; see Section 13.4.1). Nonetheless, ocean thermal expansion and heat content change simulated in CMIP5 models show relatively good agreement with observations, although this might also be due to a compensation between ocean heat uptake efficiency and atmospheric feedbacks (Kuhlbrodt and Gregory, 2012). In summary, constraints on effective ocean diffusivity are presently not conclusive.

\subsubsection{Earth System Properties}

A number of papers have found the global warming response to $\mathrm{CO}_{2}$ emissions to be determined primarily by total cumulative emissions of $\mathrm{CO}_{2}$, irrespective of the timing of those emissions over a broad range of scenarios (Allen et al., 2009; Matthews et al., 2009; Zickfeld et al., 2009; Section 12.5.4.2), although Bowerman et al. (2011) find that, when scenarios with persistent 'emission floors' are included, the strongest predictor of peak warming is cumulative emissions to 2200 . Moreover, the ratio of global warming to cumulative carbon emissions, known variously as the Absolute Global Temperature Change Potential (AGTP; defined for an infinitesimal pulse emission) (Shine et al., 2005), the Cumulative Warming Commitment (defined based on peak warming in response to a finite injection; CWC) (Allen et al., 2009) or the Carbon Climate Response (CCR) (Matthews et al., 2009), is approximately scenario-independent and constant in time.

The ratio of $\mathrm{CO}_{2}$-induced warming realized by a given year to cumulative carbon emissions to that year is known as the Transient Climate Response to cumulative $\mathrm{CO}_{2}$ Emissions (TCRE, see Chapter 12). TCRE depends on TCR and the Cumulative Airborne Fraction (CAF), which is the ratio of the increased mass of $\mathrm{CO}_{2}$ in the atmosphere to cumulative $\mathrm{CO}_{2}$ emissions (not including natural fluxes and those arising from Earth system feedbacks) over a long period, typically since pre-industrial times (Gregory et al., 2009): TCRE $=\mathrm{TCR} \times \mathrm{CAF} / \mathrm{C}_{0}$, where $\mathrm{C}_{0}$ is the mass of carbon (in the form of $\mathrm{CO}_{2}$ ) in the pre-industrial atmosphere $(590 \mathrm{PgC})$. Given estimates of $\mathrm{CAF}$ to the time of $\mathrm{CO}_{2}$ doubling of 0.4 to 0.7 (Zickfeld et al., 2013), we therefore expect values of TCRE, if expressed in units of ${ }^{\circ} \mathrm{C}$ per $1000 \mathrm{PgC}$, to be similar to or slightly lower than, and more uncertain than, values of TCR (Gillett et al., 2013 ).

TCRE may be estimated from observations by dividing an estimate of warming to date attributable to $\mathrm{CO}_{2}$ by historical cumulative carbon emissions, which gives a 5 to $95 \%$ range of $0.7^{\circ} \mathrm{C}$ to $2.0^{\circ} \mathrm{C}$ per 1000 $\mathrm{PgC}$ (Gillett et al., 2013 ), $1.0^{\circ} \mathrm{C}$ to $2.1^{\circ} \mathrm{C}$ per $1000 \mathrm{PgC}$ (Matthews et al., 2009) or $1.4^{\circ} \mathrm{C}$ to $2.5^{\circ} \mathrm{C}$ per $1000 \mathrm{PgC}$ (Allen et al., 2009), the higher range in the latter study reflecting a higher estimate of $\mathrm{CO}_{2}$-attributable warming to 2000 . The peak warming induced by a given total cumulative carbon emission (Peak Response to Cumulative Emissions (PRCE)) is less well constrained, since warming may continue even after a complete cessation of $\mathrm{CO}_{2}$ emissions, particularly in high-response models or scenarios. Using a combination of observations and models to constrain temperature and carbon cycle parameters in a simple climate-carbon-cycle model, (Allen et al., 2009), obtain a PRCE 5 to $95 \%$ confidence interval of $1.3^{\circ} \mathrm{C}$ to $3.9^{\circ} \mathrm{C}$ per $1000 \mathrm{PgC}$. They also report that (Meinshausen et al., 2009) obtain a 5 to $95 \%$ range in PRCE of $1.1^{\circ} \mathrm{C}$ to $2.7^{\circ} \mathrm{C}$ per $1000 \mathrm{PgC}$ using a Bayesian approach with a different simple model, with climate parameters constrained 
by observed warming and carbon cycle parameters constrained by the C4MIP simulations (Friedlingstein et al., 2006).

The ratio of warming to cumulative emissions, the TCRE, is assessed to be likely between $0.8^{\circ} \mathrm{C}$ and $2.5^{\circ} \mathrm{C}$ per $1000 \mathrm{PgC}$ based on observational constraints. This implies that, for warming due to $\mathrm{CO}_{2}$ emissions alone to be likely less than $2^{\circ} \mathrm{C}$ at the time $\mathrm{CO}_{2}$ emissions cease, total cumulative emissions from all anthropogenic sources over the entire industrial era would need to be limited to about $1000 \mathrm{PgC}$, or one trillion tonnes of carbon (see Section 12.5.4).

\subsection{Synthesis}

The evidence has grown since the Fourth Assessment Report that widespread changes observed in the climate system since the 1950s are attributable to anthropogenic influences. This evidence is documented in the preceding sections of this chapter, including for near surface temperatures (Section 10.3.1.1), free atmosphere temperatures (Section 10.3.1.2), atmospheric moisture content (Section 10.3.2.1), precipitation over land (Section 10.3.2.2), ocean heat content (Section 10.4.1), ocean salinity (Section 10.4.2), sea level (Section 10.4.3), Arctic sea ice (Section 10.5.1), climate extremes (Section 10.6) and evidence from the last millenium (Section 10.7). These results strengthen the conclusion that human influence on climate has played the dominant role in observed warming since the 1950s. However, the approach taken so far in this chapter has been to examine each aspect of the climate system - the atmosphere, oceans, cryosphere, extremes, and from paleoclimate archives-separately in each section and sub-section. In this section we look across the whole climate system to assess the extent that a consistent picture emerges across sub-systems and climate variables.

\subsubsection{Multi-variable Approaches}

Multi-variable studies provide one approach to gain a more comprehensive view across the climate system, although there have been relatively few applications of multi-variable detection and attribution studies in the literature. A combined analysis of near-surface temperature from weather stations and free atmosphere temperatures from radiosondes detected an anthropogenic influence on the joint changes in temperatures near the surface and aloft (Jones et al., 2003). In a Bayesian application of detection and attribution Schnur and Hasselmann (2005) combined surface temperature, diurnal temperature range and precipitation into a single analysis and showed strong net evidence for detection of anthropogenic forcings despite low likelihood ratios for diurnal temperature range and precipitation on their own. Barnett et al. (2008) applied a multi-variable approach in analysing changes in the hydrology of the Western United States (see also Section 10.3.2.3).

The potential for a multi-variable analysis to have greater power to discriminate between forced changes and internal variability has been demonstrated by Stott and Jones (2009) and Pierce et al. (2012). In the former case, they showed that a multi-variable fingerprint consisting of the responses of GMST and sub-tropical Atlantic salinity has a higher $\mathrm{S} / \mathrm{N}$ ratio than the fingerprints of each variable separately. They found reduced detection times as a result of low correlations between the two variables in the control simulation although the detection result depends on the ability of the models to represent the co-variability of the variables concerned. Multi-variable attribution studies potentially provide a stronger test of climate models than single variable attribution studies although there can be sensitivity to weighting of different components of the multi-variable fingerprint. In an analysis of ocean variables, Pierce et al. (2012) found that the joint analysis of temperature and salinity changes yielded a stronger signal of climate change than 'either salinity or temperature alone'.

Further insights can be gained by considering a synthesis of evidence across the climate system. This is the subject of the next subsection.

\subsubsection{Whole Climate System}

To demonstrate how observed changes across the climate system can be understood in terms of natural and anthropogenic causes Figure 10.21 compares observed and modelled changes in the atmosphere, ocean and cryosphere. The instrumental records associated with each element of the climate system are generally independent (see FAQ 2.1), and consequently joint interpretations across observations from the main components of the climate system increases the confidence to higher levels than from any single study or component of the climate system. The ability of climate models to replicate observed changes (to within internal variability) across a wide suite of climate indicators also builds confidence in the capacity of the models to simulate the Earth's climate.

The coherence of observed changes for the variables shown in Figure 10.21 with climate model simulations that include anthropogenic and natural forcing is remarkable. Surface temperatures over land, SSTs and ocean heat content changes show emerging anthropogenic and natural signals with a clear separation between the observed changes and the alternative hypothesis of just natural variations (Figure 10.21, Global panels). These signals appear not just in the global means, but also at continental and ocean basin scales in these variables. Sea ice emerges strongly from the range expected from natural variability for the Arctic and Antarctica remains broadly within the range of natural variability consistent with expectations from model simulations including anthropogenic forcings.

Table 10.1 illustrates a larger suite of detection and attribution results across the climate system than summarized in Figure 10.21. These results include observations from both the instrumental record and paleo-reconstructions on a range of time scales ranging from daily extreme precipitation events to variability over millennium time scales.

From up in the stratosphere, down through the troposphere to the surface of the Earth and into the depths of the oceans there are detectable signals of change such that the assessed likelihood of a detectable, and often quantifiable, human contribution ranges from likely to extremely likely for many climate variables (Table 10.1). Indeed to successfully describe the observed warming trends in the atmosphere, ocean and at the surface over the past 50 years, contributions from both anthropogenic and natural forcings are required (e.g., results 1, 2, 3, 4, 5, 7, 9 in Table 10.1). This is consistent with anthropogenic forcings warming the surface of the Earth, troposphere and oceans superimposed with cooling events caused by the three large explosive volcanic eruptions since the 1960's. These two effects (anthropogenic warming and vol- 
Frequently Asked Questions

\section{FAQ 10.2 When Will Human Influences on Climate Become Obvious on Local Scales?}

Human-caused warming is already becoming locally obvious on land in some tropical regions, especially during the warm part of the year. Warming should become obvious in middle latitudes-during summer at first-within the next several decades. The trend is expected to emerge more slowly there, especially during winter, because natural climate variability increases with distance from the equator and during the cold season. Temperature trends already detected in many regions have been attributed to human influence. Temperature-sensitive climate variables, such as Arctic sea ice, also show detected trends attributable to human influence.

Warming trends associated with global change are generally more evident in averages of global temperature than in time series of local temperature ('local' here refers generally to individual locations, or small regional averages). This is because most of the local variability of local climate is averaged away in the global mean. Multi-decadal warming trends detected in many regions are considered to be outside the range of trends one might expect from natural internal variability of the climate system, but such trends will only become obvious when the local mean climate emerges from the 'noise' of year-to-year variability. How quickly this happens depends on both the rate of the warming trend and the amount of local variability. Future warming trends cannot be predicted precisely, especially at local scales, so estimates of the future time of emergence of a warming trend cannot be made with precision.

In some tropical regions, the warming trend has already emerged from local variability (FAQ 10.2, Figure 1). This happens more quickly in the tropics because there is less temperature variability there than in other parts of the globe. Projected warming may not emerge in middle latitudes until the mid-21st century-even though warming trends there are larger - because local temperature variability is substantially greater there than in the tropics. On a seasonal basis, local temperature variability tends to be smaller in summer than in winter. Warming therefore tends to emerge first in the warm part of the year, even in regions where the warming trend is larger in winter, such as in central Eurasia in FAQ 10.2, Figure 1.

Variables other than land surface temperature, including some oceanic regions, also show rates of long-term change different from natural variability. For example, Arctic sea ice extent is declining very rapidly, and already shows a human influence. On the other hand, local precipitation trends are very hard to detect because at most locations the variability in precipitation is quite large. The probability of record-setting warm summer temperatures has increased throughout much of the Northern Hemisphere. High temperatures presently considered extreme are projected to become closer to the norm over the coming decades. The probabilities of other extreme events, including some cold spells, have lessened.

In the present climate, individual extreme weather events cannot be unambiguously ascribed to climate change, since such events could have happened in an unchanged climate. However the probability of occurrence of such events could have changed significantly at a particular location. Human-induced increases in greenhouse gases are estimated to have contributed substantially to the probability of some heatwaves. Similarly, climate model studies suggest that increased greenhouse gases have contributed to the observed intensification of heavy precipitation events found over parts of the Northern Hemisphere. However, the probability of many other extreme weather events may not have changed substantially. Therefore, it is incorrect to ascribe every new weather record to climate change.

The date of future emergence of projected warming trends also depends on local climate variability, which can temporarily increase or decrease temperatures. Furthermore, the projected local temperature curves shown in FAQ 10.2, Figure 1 are based on multiple climate model simulations forced by the same assumed future emissions scenario. A different rate of atmospheric greenhouse gas accumulation would cause a different warming trend, so the spread of model warming projections (the coloured shading in FAQ 10.2, Figure 1) would be wider if the figure included a spread of greenhouse gas emissions scenarios. The increase required for summer temperature change to emerge from 20th century local variability (regardless of the rate of change) is depicted on the central map in FAQ 10.2, Figure 1.

A full answer to the question of when human influence on local climate will become obvious depends on the strength of evidence one considers sufficient to render something 'obvious'. The most convincing scientific evidence for the effect of climate change on local scales comes from analysing the global picture, and from the wealth of evidence from across the climate system linking many observed changes to human influence. (continued on next page) 

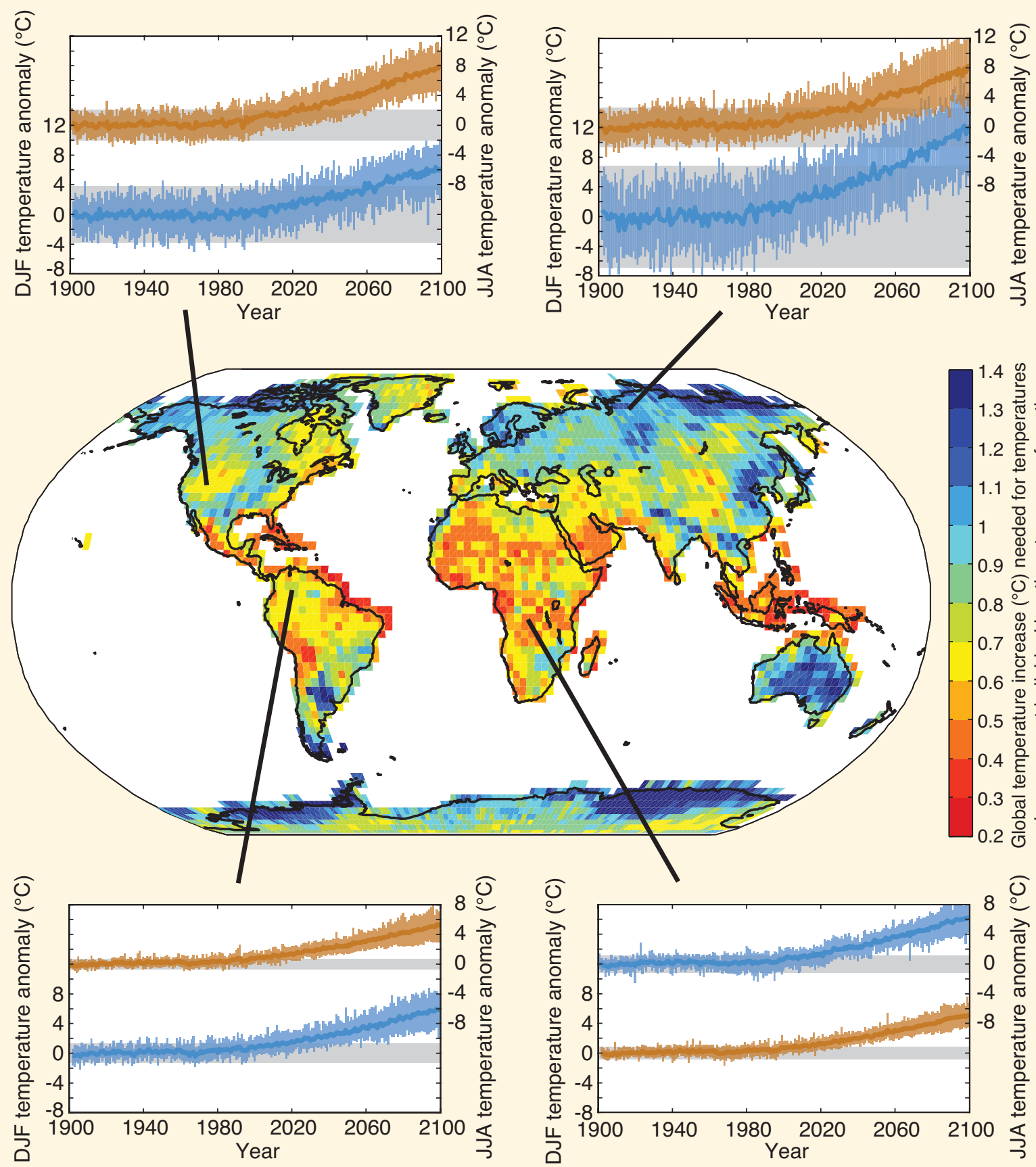

FAQ 10.2, Figure 1 | Time series of projected temperature change shown at four representative locations for summer (red curves, representing June, July and August at sites in the tropics and Northern Hemisphere or December, January and February in the Southern Hemisphere) and winter (blue curves). Each time series is surrounded by an envelope of projected changes (pink for the local warm season, blue for the local cold season) yielded by 24 different model simulations, emerging from a grey envelope of natural local variability simulated by the models using early 20th century conditions. The warming signal emerges first in the tropics during summer. The central map shows the global temperature increase $\left({ }^{\circ} \mathrm{C}\right)$ needed for temperatures in summer at individual locations to emerge from the envelope of early 20th century variability. Note that warm colours denote the smallest needed temperature increase, hence earliest time of emergence. All calculations are based on Coupled Model Intercomparison Project Phase 5 (CMIP5) global climate model simulations forced by the Representative Concentration Pathway 8.5 (RCP8.5) emissions scenario. Envelopes of projected change and natural variability are defined as \pm 2 standard deviations. (Adapted and updated from Mahlstein et al., 2011.) 


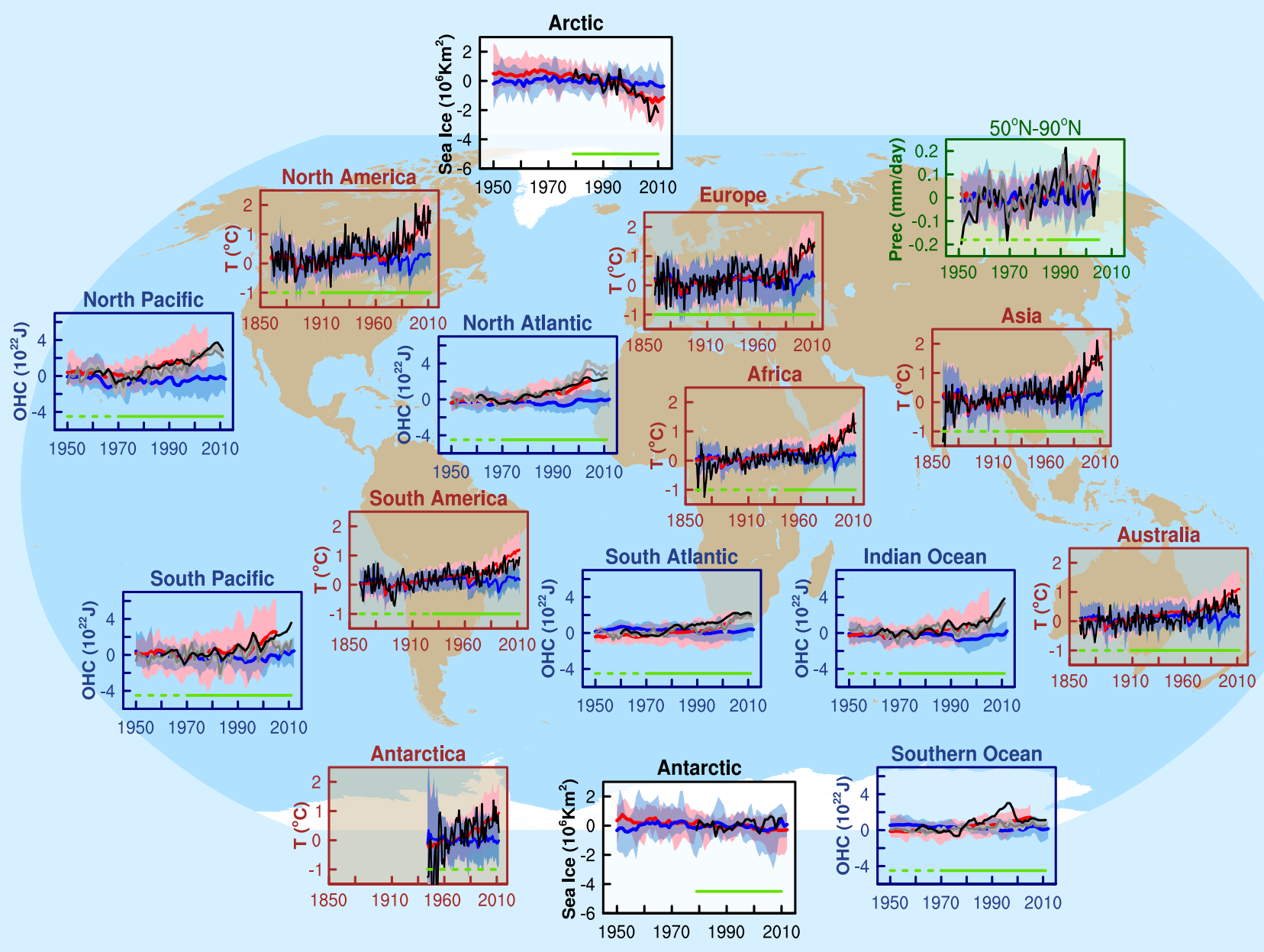

\section{Global}
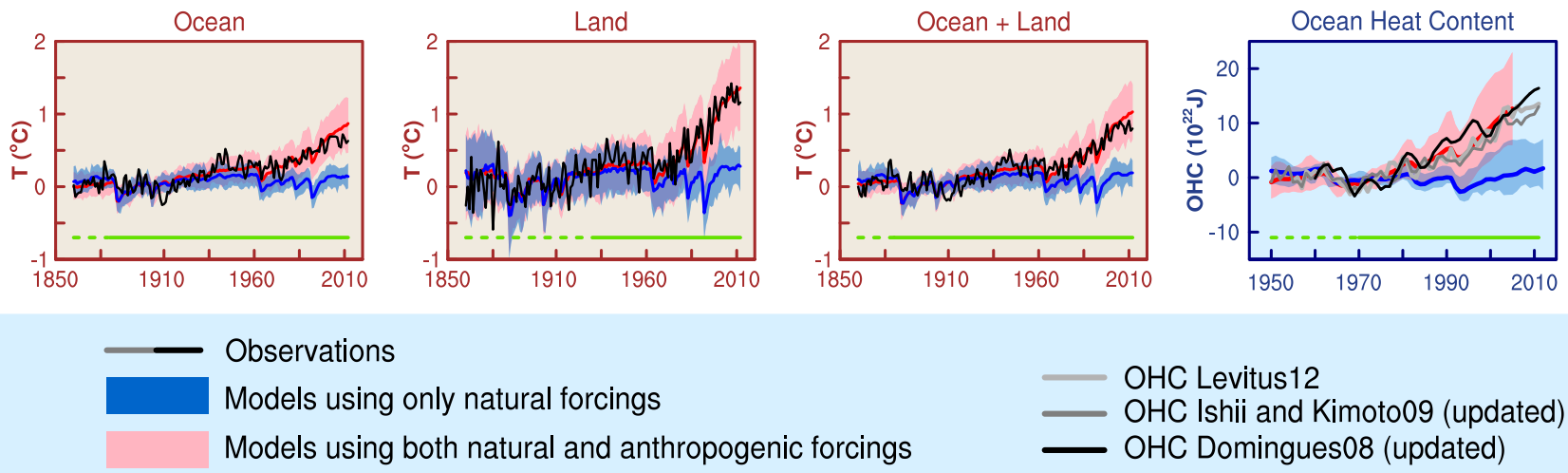

OHC Levitus 12

OHC Ishii and Kimoto09 (updated)

OHC Domingues08 (updated)

Figure 10.21 | Detection and attribution signals in some elements of the climate system, at regional scales (top panels) and global scales (bottom four panels). Brown panels are land surface temperature time series, green panels are precipitation time series, blue panels are ocean heat content time series and white panels are sea ice time series. Observations are shown on each panel in black or black and shades of grey. Blue shading is the model time series for natural forcing simulations and pink shading is the combined natural and anthropogenic forcings. The dark blue and dark red lines are the ensemble means from the model simulations. All panels show the 5 to $95 \%$ intervals of the natural forcing simulations, and the natural and anthropogenic forcing simulations. For surface temperature the results are from Jones et al. (2013) (and Figure 10.1). The observed surface temperature is from Hadley Centre/Climatic Research Unit gridded surface temperature data set 4 (HadCRUT4). Observed precipitation is from Zhang et al. (2007) (black line) and CRU TS 3.0 updated (grey line). Three observed records of ocean heat content (OHC) are shown. Sea ice anomalies (rather than absolute values) are plotted and based on models in Figure 10.16. The green horizontal lines indicate quality of the observations and estimates. For land and ocean surface temperatures panels and precipitation panels, solid green lines at bottom of panels indicate where data spatial coverage being examined is above $50 \%$ coverage and dashed green lines where coverage is below $50 \%$. For example, data coverage of Antarctica never goes above $50 \%$ of the land area of the continent. For ocean heat content and sea ice panels the solid green line is where the coverage of data is good and higher in quality, and the dashed green line is where the data coverage is only adequate. More details of the sources of model simulations and observations are given in the Supplementary Material (10.SM.1). 
canic eruptions) cause much of the observed response (see also Figures $10.5,10.6,10.9,10.14 a$ and 10.21). Both natural and anthropogenic forcings are required to understand fully the variability of the Earth system during the past 50 years.

Water in the free atmosphere is expected to increase, as a consequence of warming of the atmosphere (Section 10.6.1), and atmospheric circulation controls the global distribution of precipitation and evaporation. Simulations show that GHGs increase moisture in the atmosphere and change its transport in such a way as to produce patterns of precipitation and evaporation that are quite distinct from the observed patterns of warming. Our assessment shows that anthropogenic forcings have contributed to observed increases in moisture content in the atmosphere (result 16, medium confidence, Table 10.1), to global scale changes in precipitation patterns over land (result 14, medium confidence), to a global scale intensification of heavy precipitation in land regions where there observational coverage is sufficient to make an assessment (result 15, medium confidence), and to changes in surface and sub-surface ocean salinity (result 11, very likely). Combining evidence from both atmosphere and ocean that systematic changes in precipitation over land and ocean salinity can be attributed to human influence supports an assessment that it is likely that human influence has affected the global water cycle since 1960.

Warming of the atmosphere and the oceans affects the cryosphere, and in the case of snow and sea ice warming leads to positive feedbacks that amplify the warming response in the atmosphere and oceans. Retreat of mountain glaciers has been observed with an anthropogenic influence detected (result 17, likely, Table 10.1), Greenland ice sheet has melted at the edges and accumulating snow at the higher elevations is consistent with GHG warming supporting an assessment for an anthropogenic influence on the negative surface mass balance of Greenland's ice sheet (result 18, likely, Table 10.1). Our level of scientific understanding is too low to provide a quantifiable explanation of the observed mass loss of the Antarctic ice sheet (low confidence, result 19, Table 10.1). Sea ice in the Arctic is decreasing rapidly and the changes now exceed internal variability and with an anthropogenic contribution detected (result 20, very likely, Table 10.1). Antarctic sea ice extent has grown overall over the last 30 years but there is low scientific understanding of the spatial variability and changes in Antarctic sea ice extent (result 21, Table 10.1). There is evidence for an anthropogenic component to observed reductions in NH snow cover since the 1970s (likely, result 22, Table 10.1).

Anthropogenic forcing has also affected temperature on continental scales, with human influences having made a substantial contribution to warming in each of the inhabited continents (results 28, likely, Table 10.1), and having contributed to the very substantial Arctic warming over the past 50 years (result 29, likely, Table 10.1) while because of large observational uncertainties there is low confidence in attribution of warming averaged over available stations over Antarctica (result 30, Table 10.1). There is also evidence that anthropogenic forcings have contributed to temperature change in many sub-continental regions (result 32, likely, Table 10.1) and that anthropogenic forcings have contributed to the observed changes in the frequency and intensity of daily temperature extremes on the global scale since the mid-20th century (result 8, very likely, Table 10.1). Furthermore there is evidence that human influence has substantially increased the probability of occurrence of heat waves in some locations (result 33, likely, Table 10.1).

An analysis of these results (from Table 10.1) shows that there is high confidence in attributing many aspects of changes in the climate system to human influence including from atmospheric measurements of temperature. Synthesizing the results in Table 10.1 shows that the combined evidence from across the climate system increases the level of confidence in the attribution of observed climate change to human influence and reduces the uncertainties associated with assessments based on a single variable. From this combined evidence, it is virtually certain that human influence has warmed the global climate system.

\section{Acknowledgements}

We acknowledge the major contributions of the following scientists who took a substantial part in the production of key figures: Beena Balan Sarojini, Oliver Browne, Jara Imbers Quintana, Gareth Jones, Fraser Lott, Irina Mahlstein, Alexander Otto, Debbie Polson, Andrew Schurer, Lijun Tao, and Muyin Wang. We also acknowledge the contributions of Viviane Vasconcellos de Menezes for her work on the production of figures and for her meticulous management of the bibliography database used for this chapter. 


\begin{tabular}{|c|c|c|c|c|}
\hline 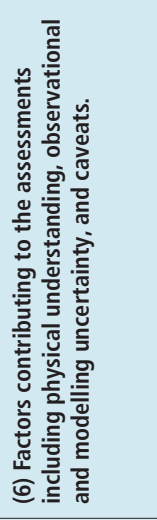 & 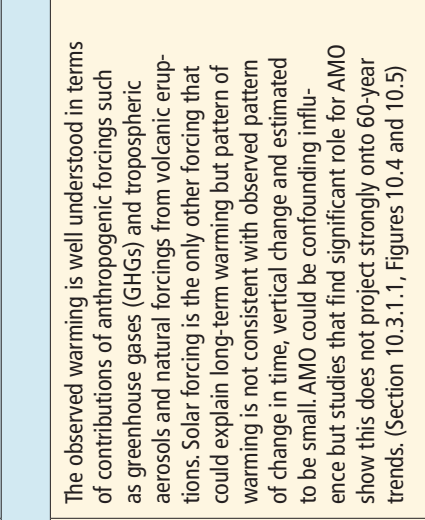 & 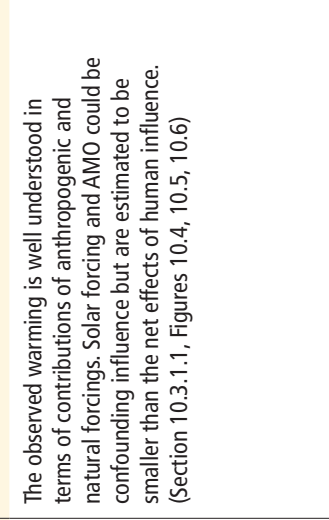 & 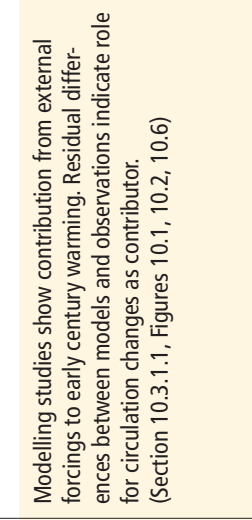 & 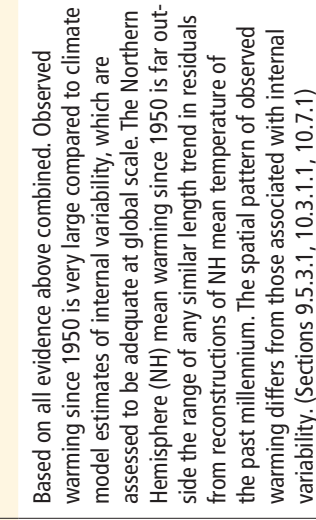 \\
\hline 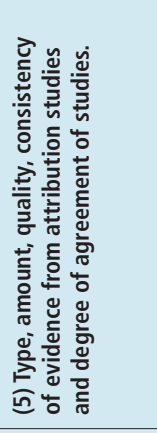 & 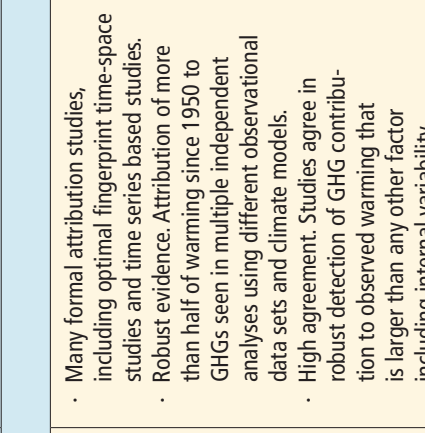 & 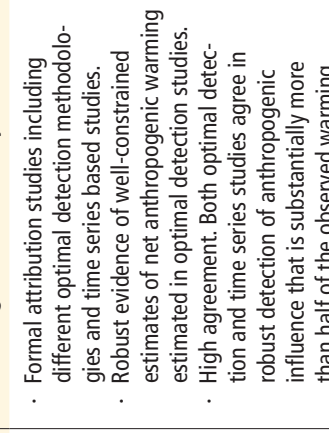 & 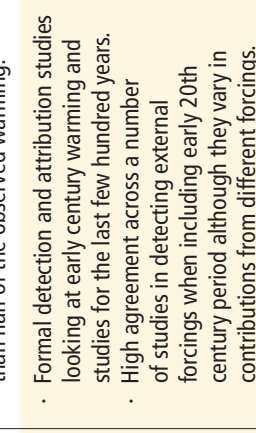 & 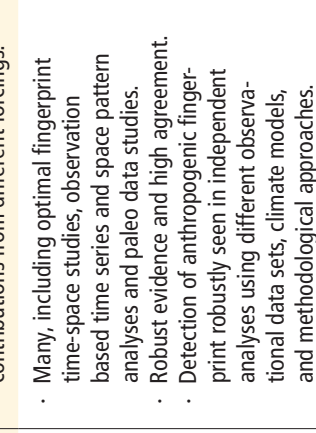 \\
\hline 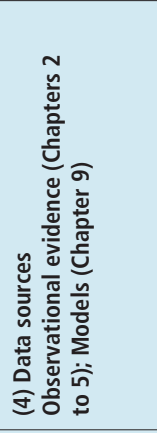 & 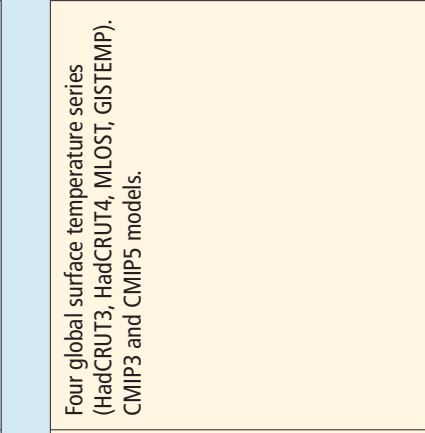 & 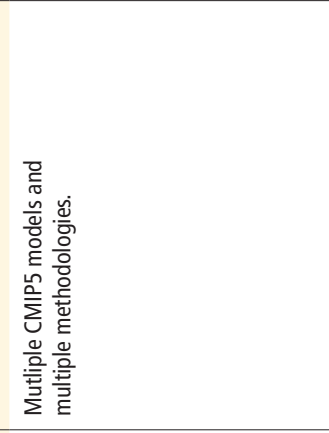 & 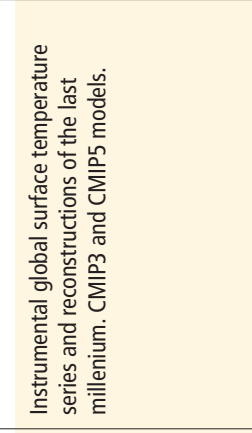 & 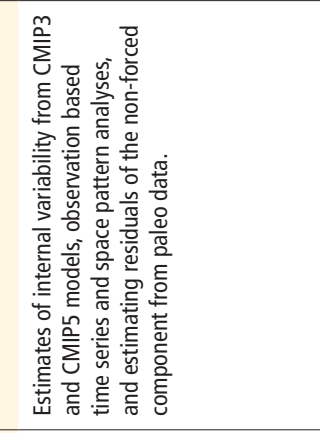 \\
\hline 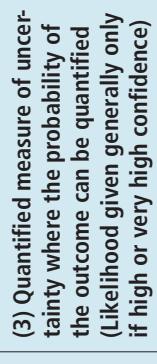 & 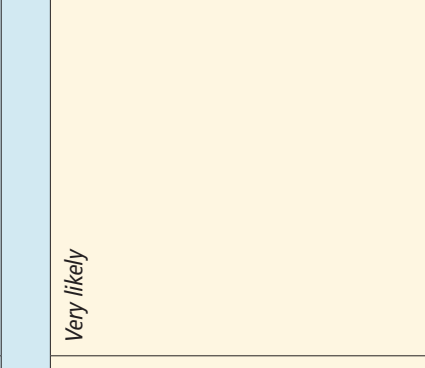 & 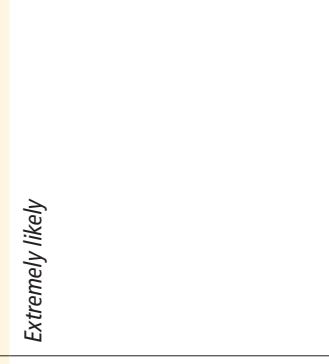 & 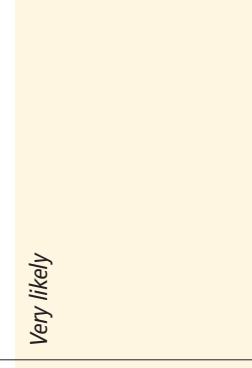 & 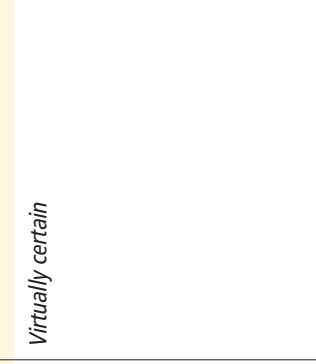 \\
\hline 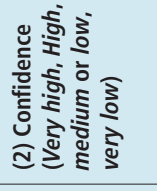 & 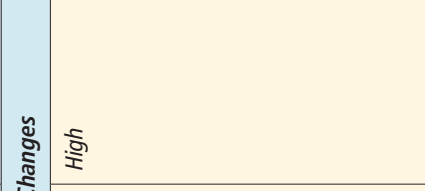 & 产 & 铃 & 䲞 \\
\hline 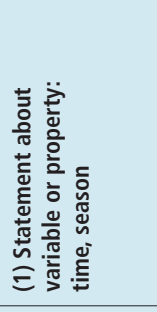 & 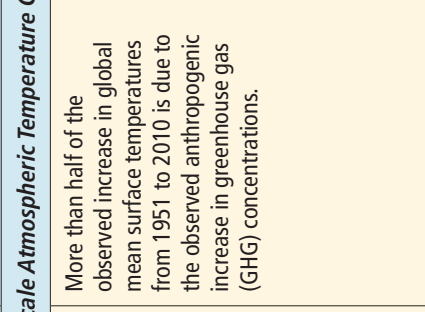 & 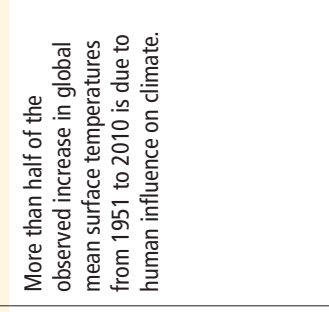 & 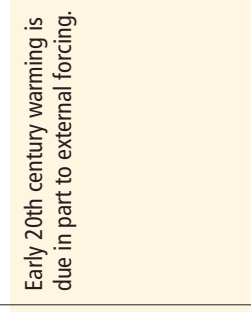 & 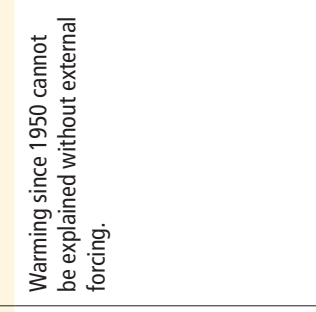 \\
\hline 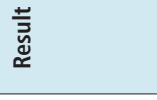 & \begin{tabular}{l|l}
$\frac{1}{0}$ \\
$\frac{0}{0}$ \\
$\frac{8}{6}$ & -
\end{tabular} & & & \\
\hline
\end{tabular}




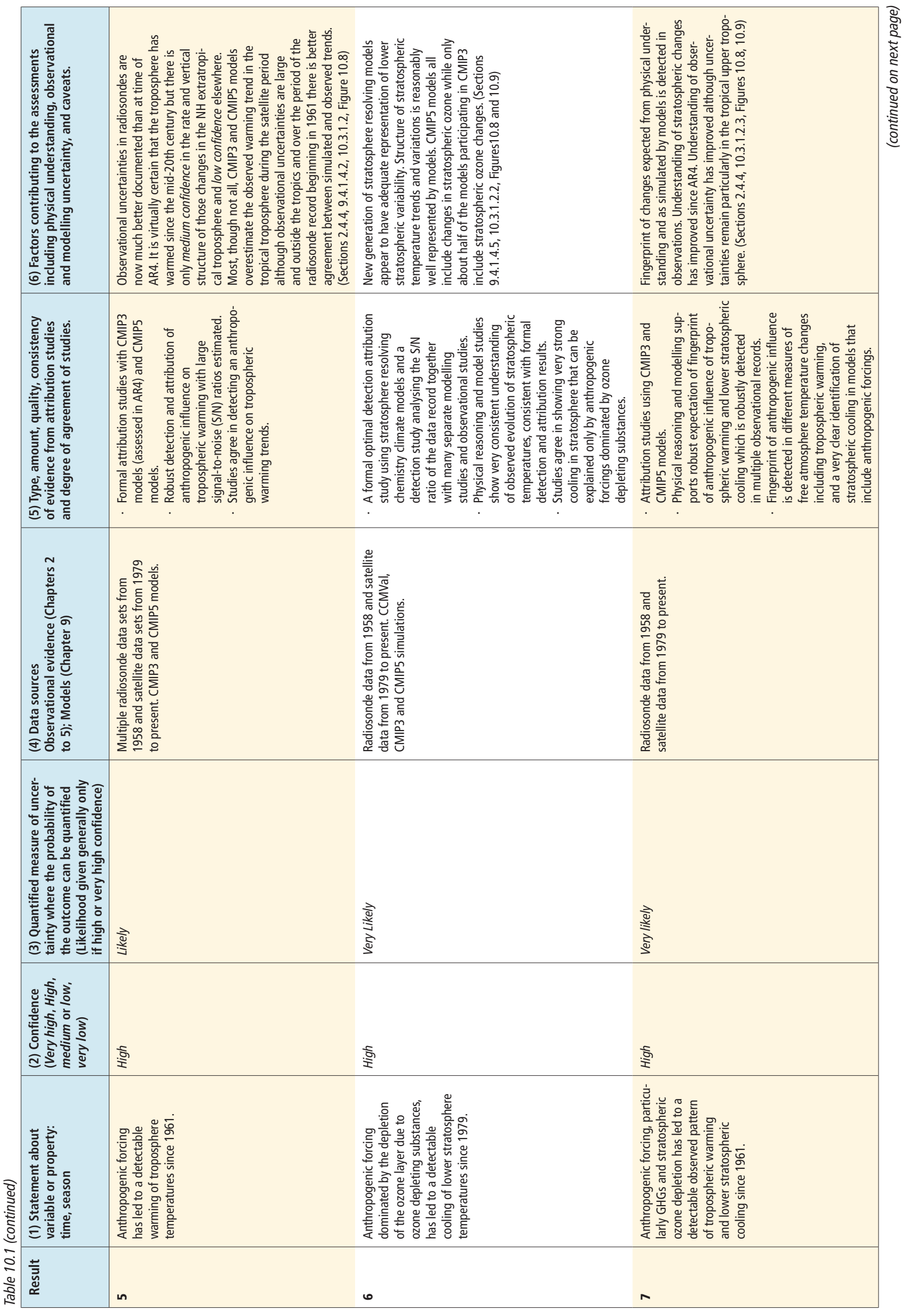




\begin{tabular}{|c|c|c|c|}
\hline 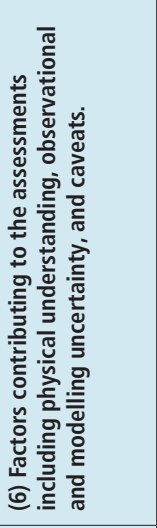 & 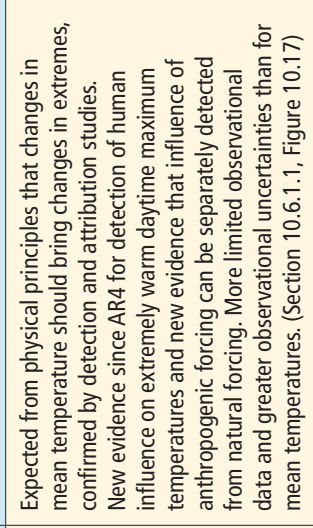 & 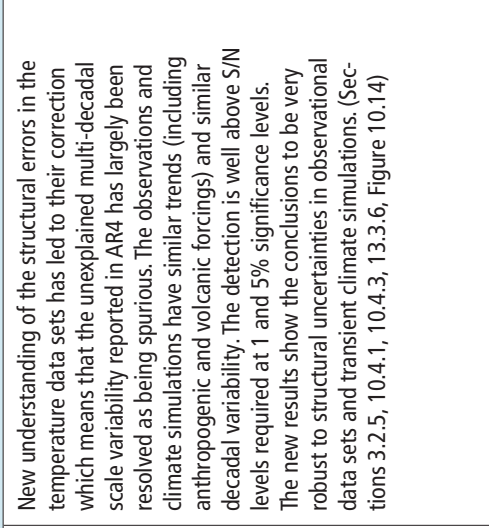 & 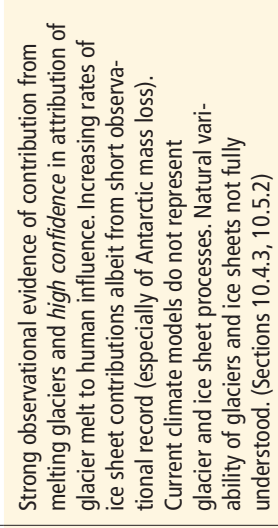 \\
\hline 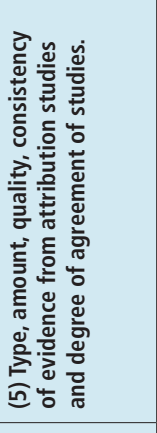 & 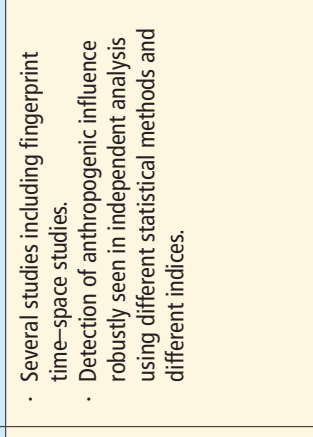 & 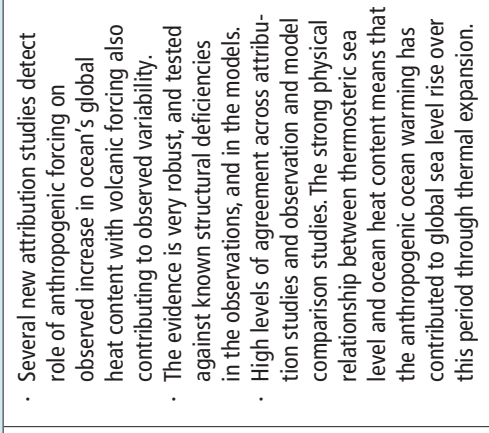 & 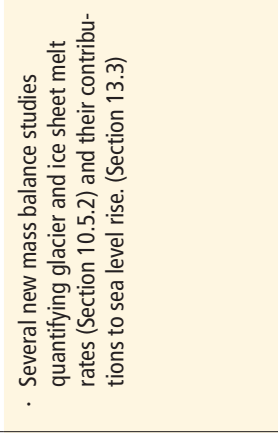 \\
\hline 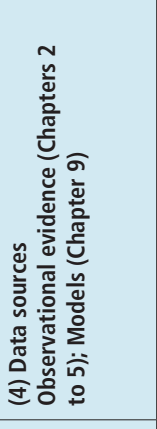 & 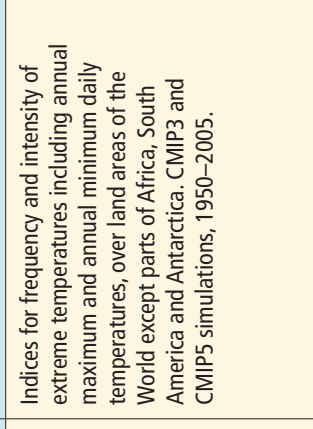 & 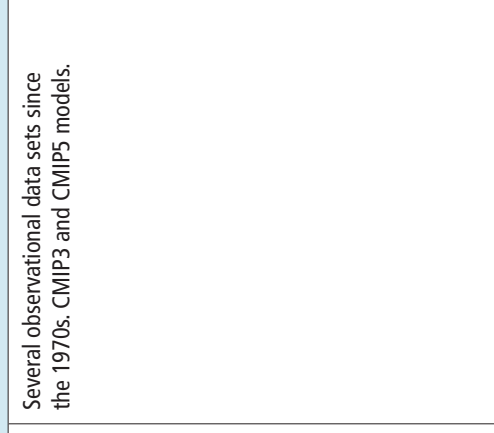 & 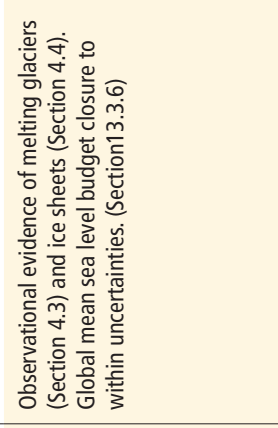 \\
\hline 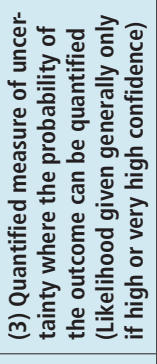 & 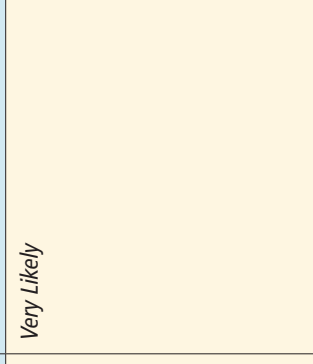 & 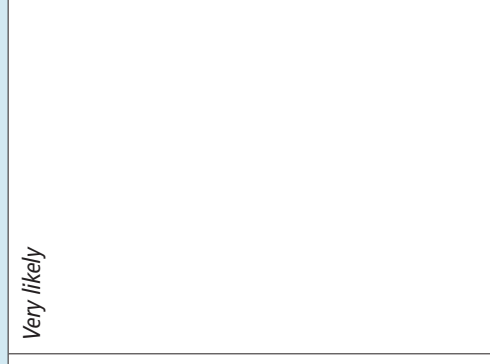 & 㨱 \\
\hline 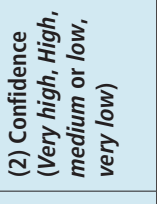 & 网 & 产 & 率 \\
\hline 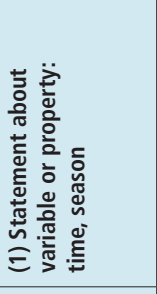 & 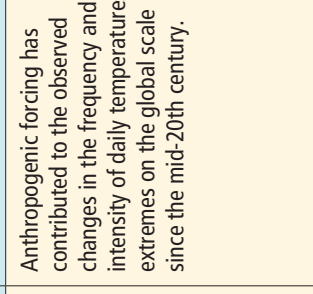 & 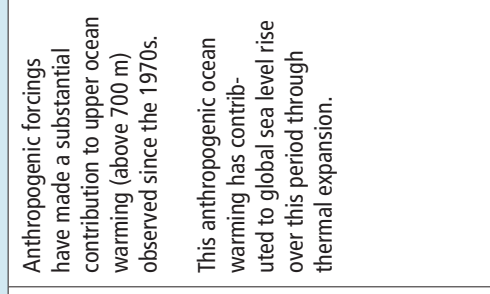 & 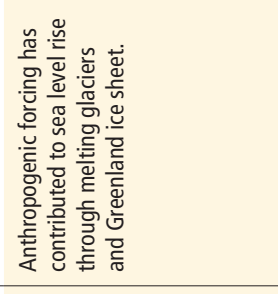 \\
\hline 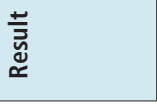 & $\infty$ & $a$ & $\stackrel{\circ}{\circ}$ \\
\hline
\end{tabular}




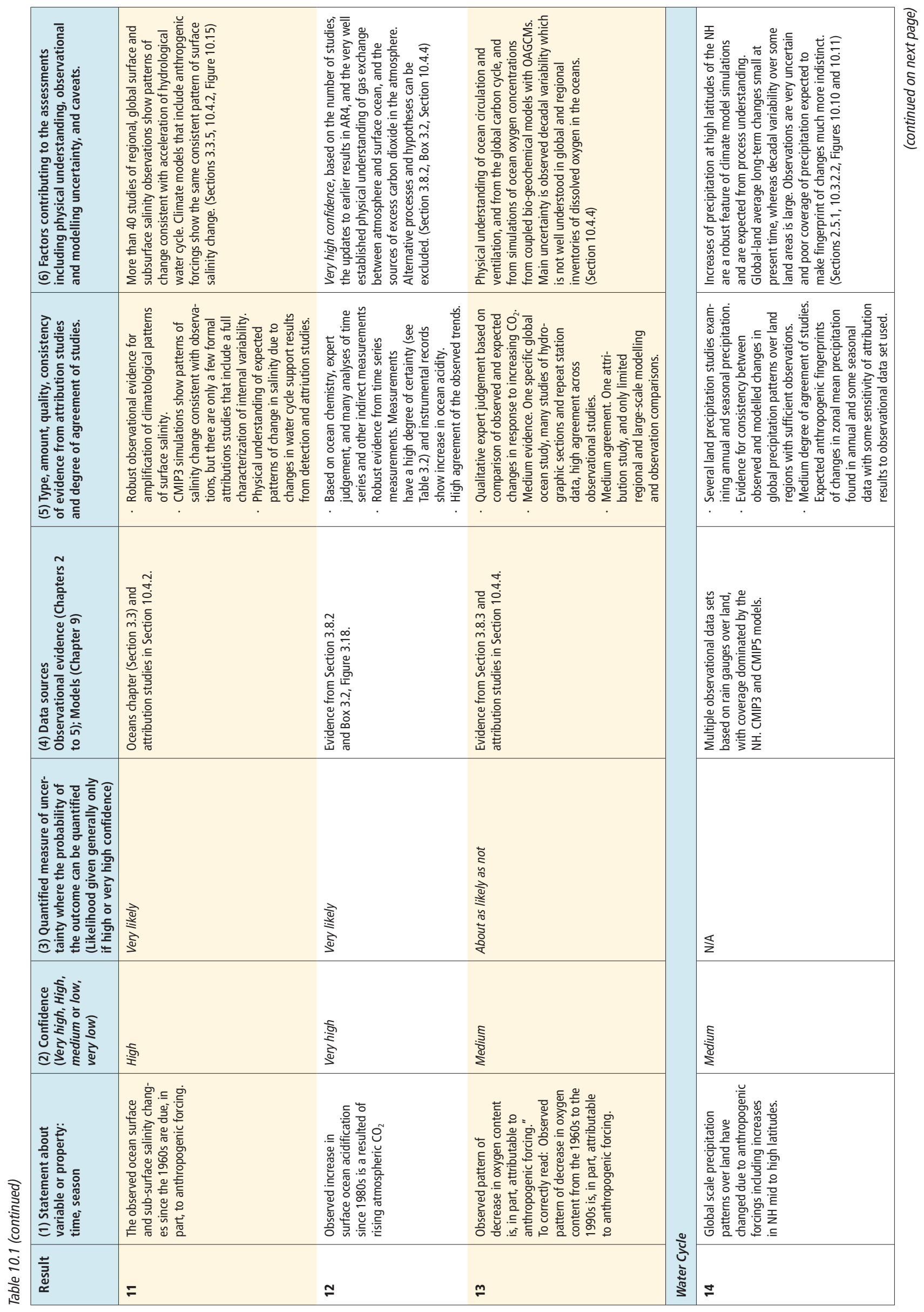




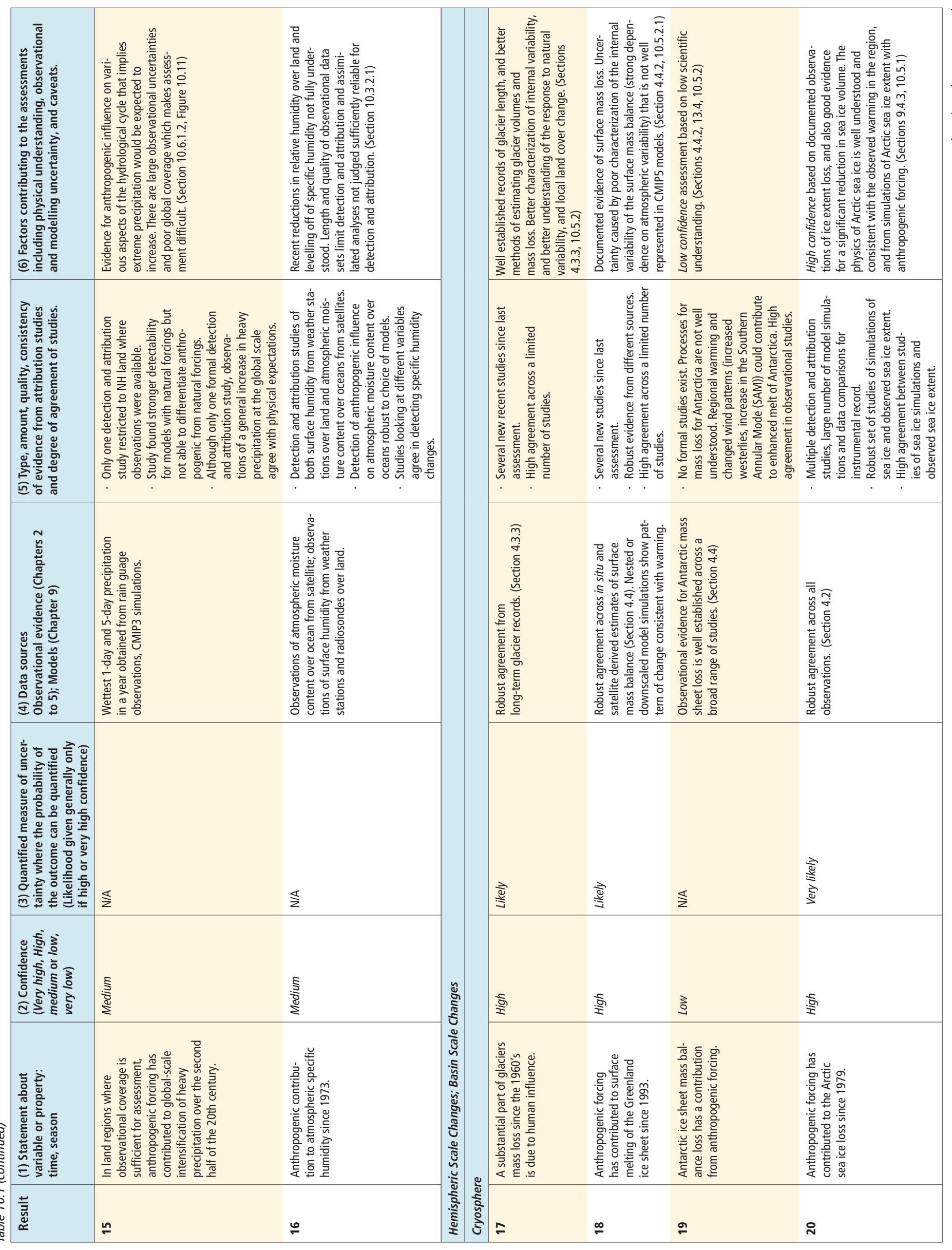




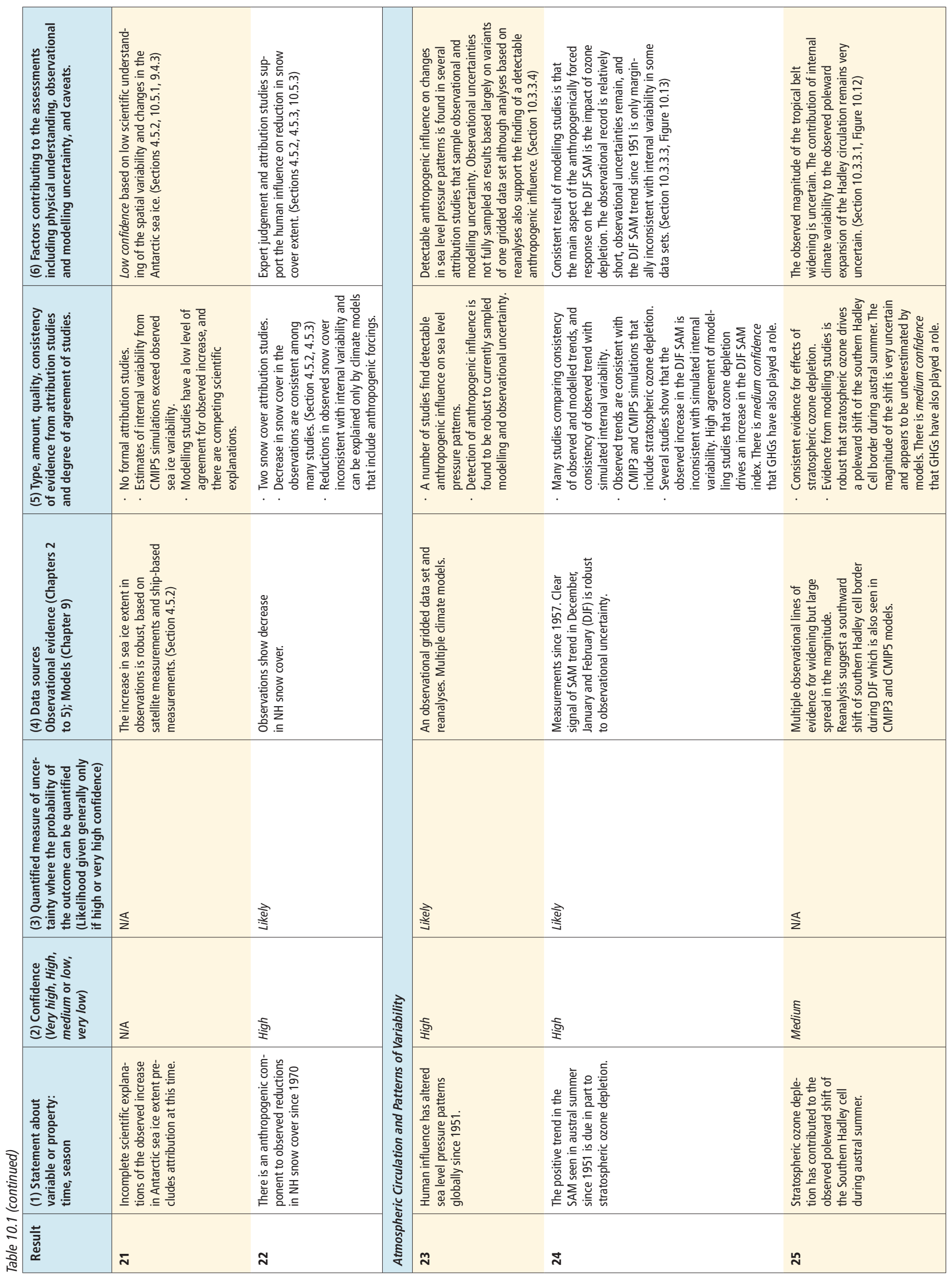



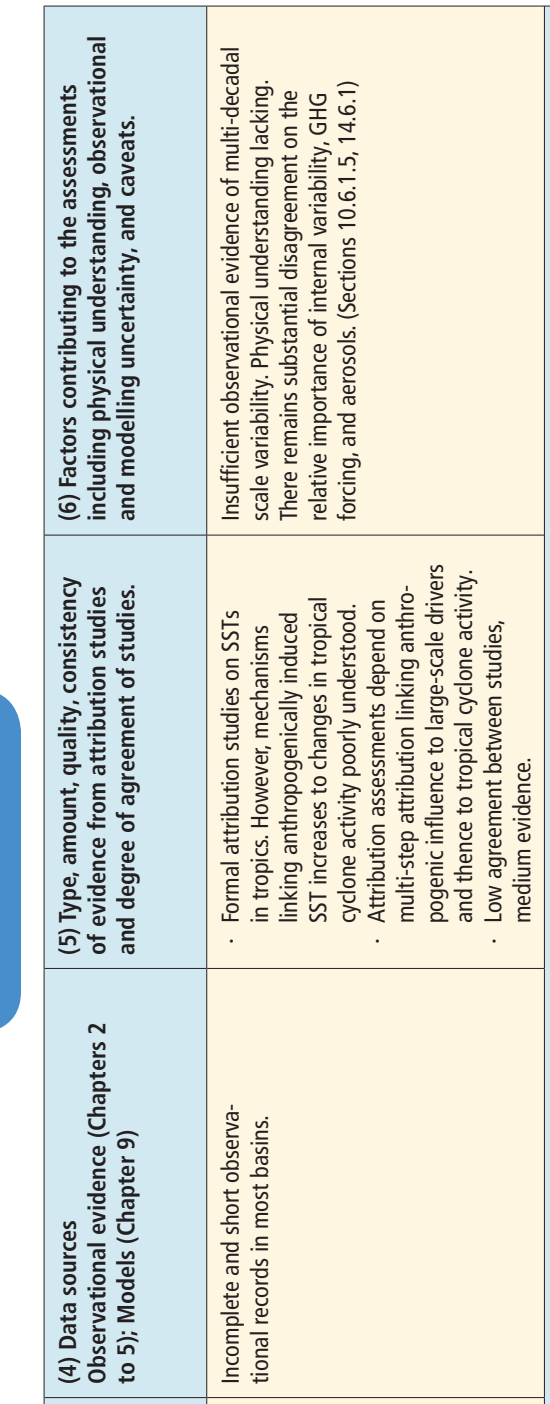

(1)
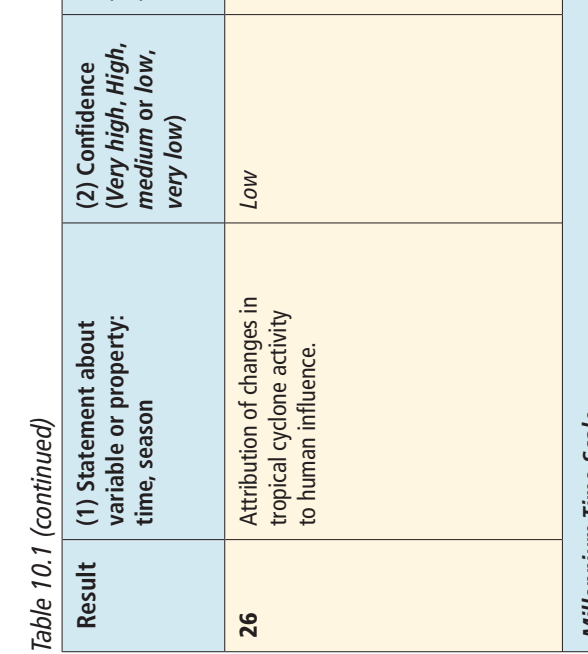

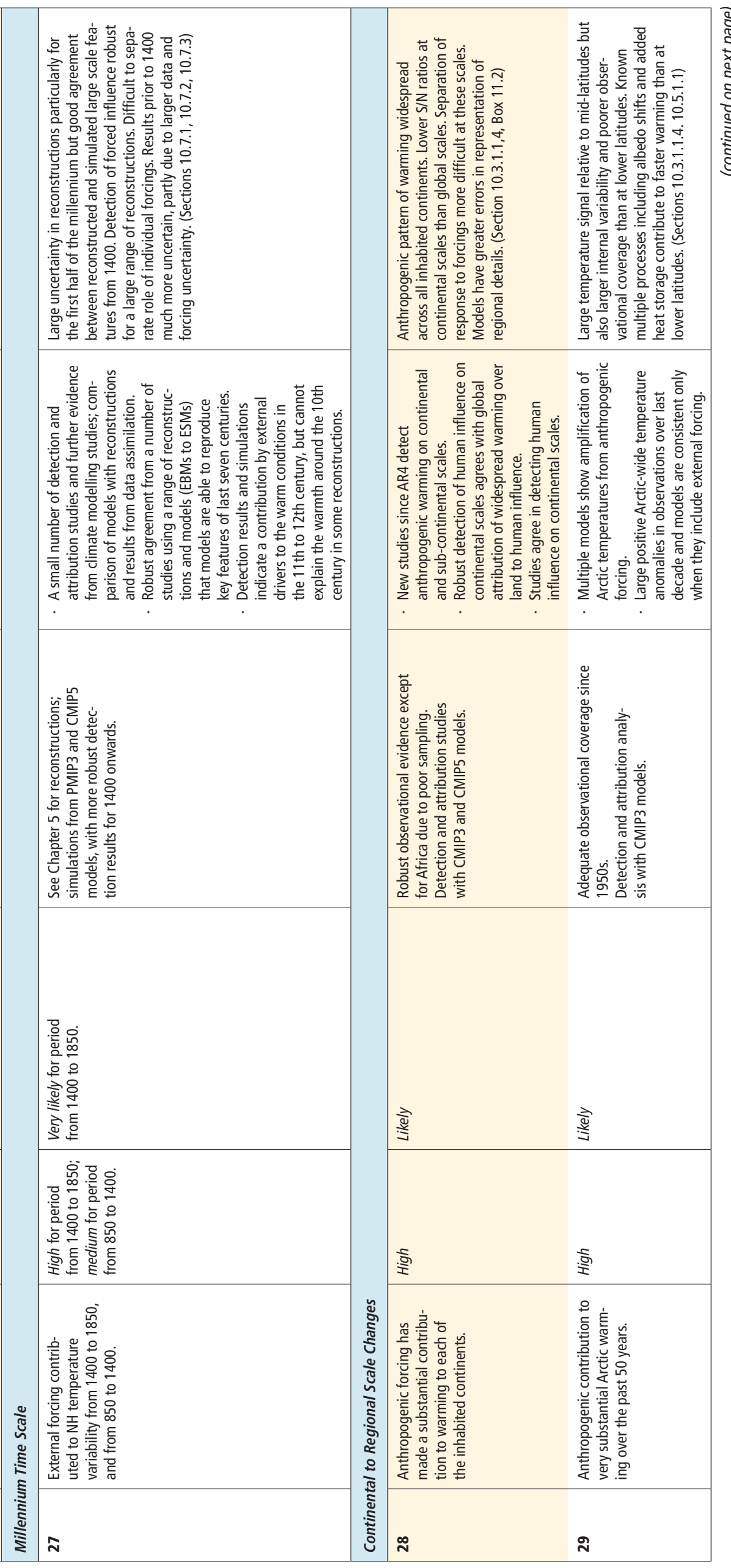




\begin{tabular}{|c|c|c|c|c|}
\hline 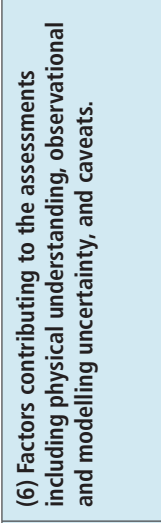 & 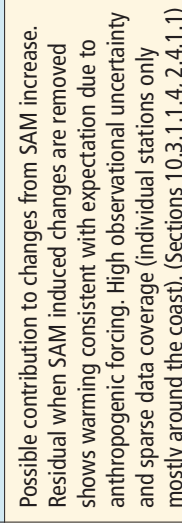 & 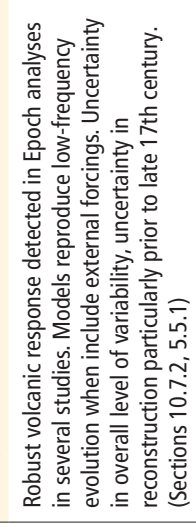 & 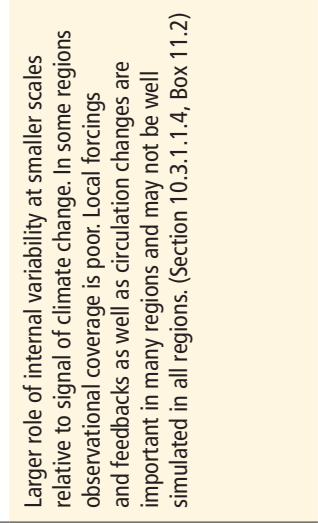 & 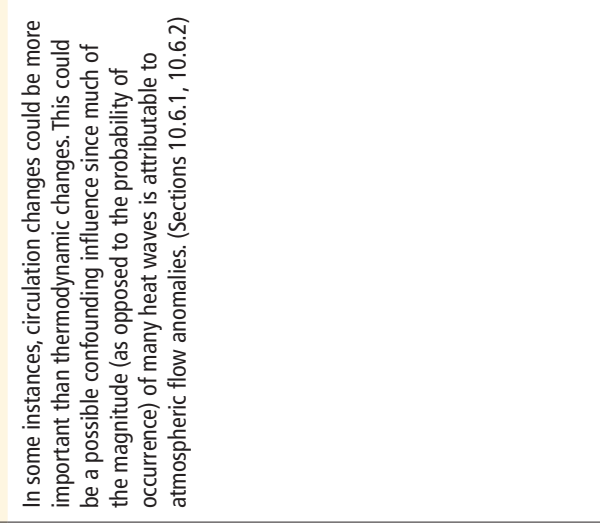 \\
\hline 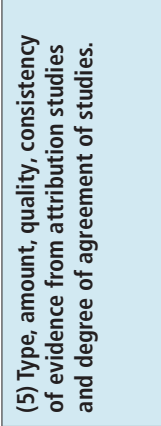 & 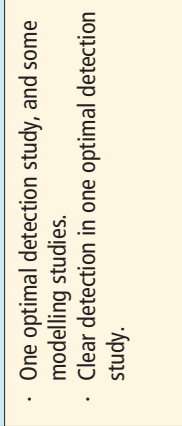 & 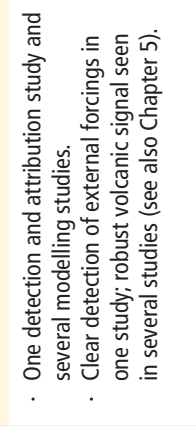 & 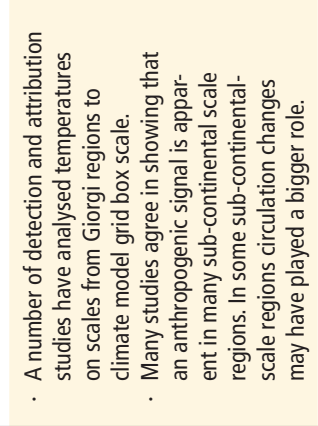 & 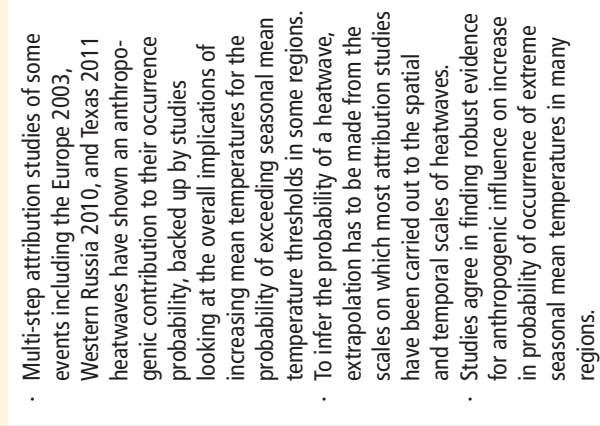 \\
\hline 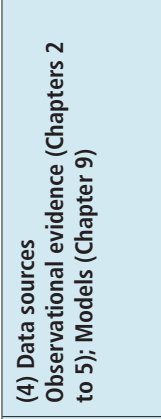 & 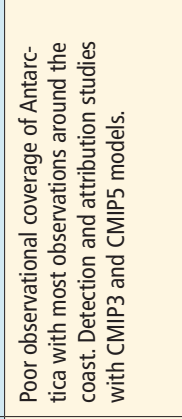 & 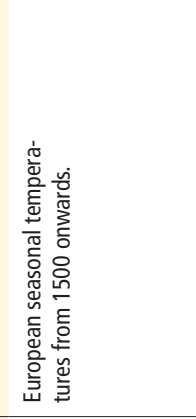 & 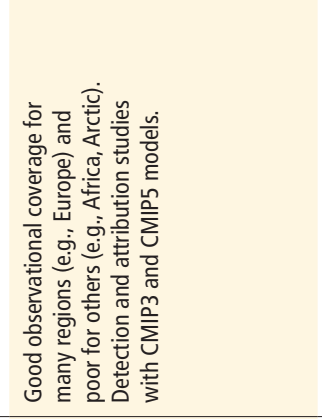 & 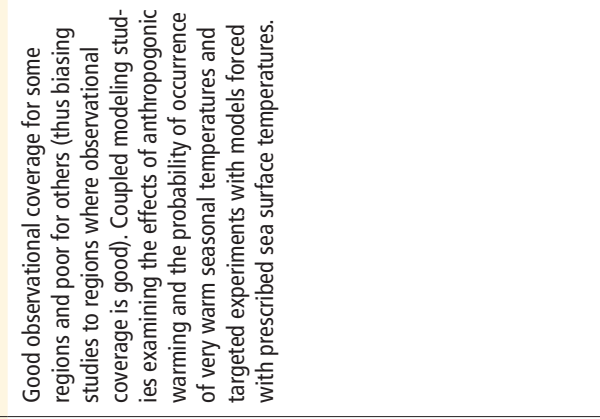 \\
\hline 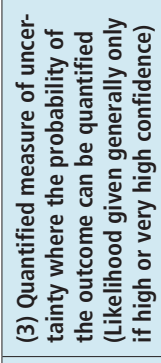 & $\frac{\pi}{z}$ & $\frac{\pi}{z}$ & 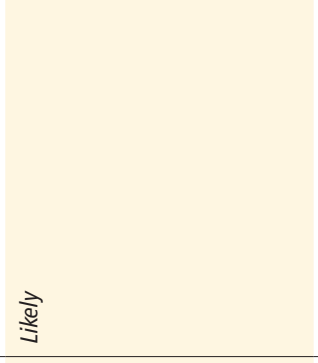 & 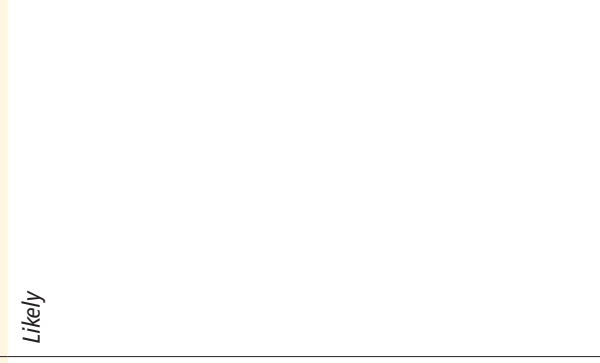 \\
\hline 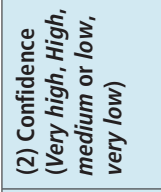 & s & 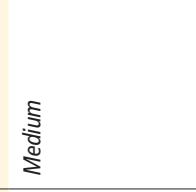 & ఫิ & 䲞 \\
\hline 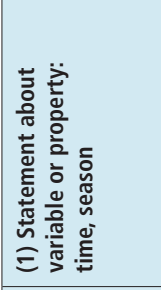 & 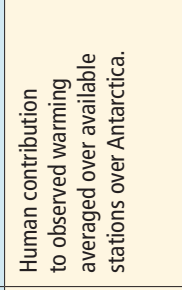 & 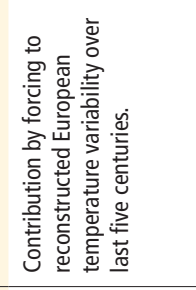 & 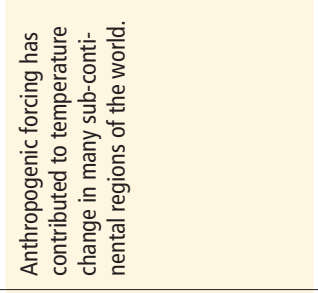 & 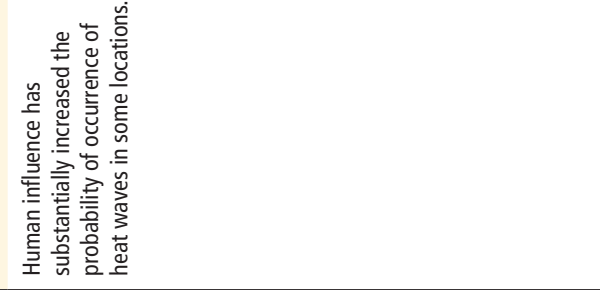 \\
\hline $\begin{array}{l}\overline{\overline{\bar{u}}} \\
\stackrel{4}{u}\end{array}$ & లి & $\bar{m}$ & $\tilde{m}$ & $\tilde{m}$ \\
\hline
\end{tabular}




\section{References}

AchutaRao, K. M., B. D. Santer, P. J. Gleckler, K. E. Taylor, D. W. Pierce, T. P. Barnett, and T. M. L. Wigley, 2006: Variability of ocean heat uptake: Reconciling observations and models. J. Geophys. Res. Oceans, 111, C05019.

AchutaRao, K. M., et al., 2007: Simulated and observed variability in ocean temperature and heat content. Proc. Natl. Acad. Sci. U.S.A., 104, 10768-10773.

Ahlmann, H. W., 1948: The present climatic fluctuation. Geogr.J., 112, 165-195.

Aldrin, M., M. Holden, P. Guttorp, R. B. Skeie, G. Myhre, and T. K. Berntsen, 2012: Bayesian estimation of climate sensitivity based on a simple climate model fitted to observations of hemispheric temperatures and global ocean heat content. Environmetrics, 23, 253-271.

Alekseev, G. V., A. I. Danilov, V. M. Kattsov, S. I. Kuz'mina, and N. E. Ivanov, 2009: Changes in the climate and sea ice of the Northern Hemisphere in the 20th and 21 st centuries from data of observations and modeling. Izvestiya Atmospheric and Oceanic Physics, 45, 675-686.

Alexander, L. V., and J. M. Arblaster, 2009: Assessing trends in observed and modelled climate extremes over Australia in relation to future projections. Int. J. Climatol., 29, 417-435.

Allan, R., and T. Ansell, 2006: A new globally complete monthly historical gridded mean sea level pressure dataset (HadSLP2): 1850-2004. J. Clim., 19, 5816-5842.

Allan, R. P., B. J. Soden, V. O. John, W. Ingram, and P. Good, 2010: Current changes in tropical precipitation. Environ. Res. Lett., 5, 025205.

Allen, M., 2011: In defense of the traditional null hypothesis: Remarks on the Trenberth and Curry WIREs opinion articles. WIREs Clim. Change, 2, 931-934.

Allen, M. R., and S. F. B. Tett, 1999: Checking for model consistency in optimal fingerprinting. Clim. Dyn., 15, 419-434.

Allen, M. R., and W. J. Ingram, 2002: Constraints on future changes in climate and the hydrologic cycle. Nature, 419, 224-232.

Allen, M. R., and P. A. Stott, 2003: Estimating signal amplitudes in optimal fingerprinting. Part I: Theory. Clim. Dyn., 21, 477-491.

Allen, M. R., P. A. Stott, J. F. B. Mitchell, R. Schnur, and T. L. Delworth, 2000: Quantifying the uncertainty in forecasts of anthropogenic climate change. Nature, 407, 617-620.

Allen, M. R., D. J. Frame, C. Huntingford, C. D. Jones, J. A. Lowe, M. Meinshausen, and N. Meinshausen, 2009: Warming caused by cumulative carbon emissions towards the trillionth tonne. Nature, 458, 1163-1166.

Allen, M. R., et al., 2006: Quantifying anthropogenic influence on recent near-surface temperature change. Surv. Geophys., 27, 491-544.

Allen, R. J., S. C. Sherwood, J. R. Norris, and C. S. Zender, 2012: Recent Northern Hemisphere tropical expansion primarily driven by black carbon and tropospheric ozone. Nature, 485, 350-354.

Ammann, C. M., and E. R. Wahl, 2007: The importance of the geophysical context in statistical evaluations of climate reconstruction procedures. Clim. Change, 85 71-88.

Ammann, C. M., F. Joos, D. S. Schimel, B. L. Otto-Bliesner, and R. A. Tomas, 2007: Solar influence on climate during the past millennium: Results from transient simulations with the NCAR Climate System Model. Proc. Natl. Acad. Sci. U.S.A., 104, 3713-3718.

Andreae, M. O., C. D. Jones, and P. M. Cox, 2005: Strong present-day aerosol cooling implies a hot future. Nature, 435, 1187-1190.

Andrews, O. D., N. L. Bindoff, P. R. Halloran, T. llyina, and C. Le Quéré, 2013: Detecting an external influence on recent changes in oceanic oxygen using an optimal fingerprinting method. Biogeosciences, 10, 1799-1813.

Annan, J. D., and J. C. Hargreaves, 2006: Using multiple observationally-based constraints to estimate climate sensitivity. Geophys. Res. Lett., 33, L06704.

Annan, J. D., and J. C. Hargreaves, 2010: Reliability of the CMIP3 ensemble. Geophys. Res. Lett., 37, L02703.

Annan, J. D., and J. C. Hargreaves, 2011: On the generation and interpretation of probabilistic estimates of climate sensitivity. Clim. Change, 104, 423-436.

Aoki, S., N. L. Bindoff, and J. A. Church, 2005: Interdecadal water mass changes in the Southern Ocean between 30 degrees E and 160 degrees E. Geophys. Res. Lett., 32, L07607.

Arkin, P. A., T. M. Smith, M. R. P. Sapiano, and J. Janowiak, 2010: The observed sensitivity of the global hydrological cycle to changes in surface temperature. Environ. Res. Lett., 5, 035201.

Bal, S., S. Schimanke, T. Spangehl, and U. Cubasch, 2011: On the robustness of the solar cycle signal in the Pacific region. Geophys. Res. Lett., 38, L14809.
Balachandran, N. K., D. Rind, P. Lonergan, and D. T. Shindell, 1999: Effects of solar cycle variability on the lower stratosphere and the troposphere. J. Geophys. Res. Atmos., 104, 27321-27339.

Balan Sarojini, B., P. Stott, E. Black, and D. Polson, 2012 Fingerprints of changes in annual and seasonal precipitation from CMIP5 models over land and ocean. Geophys. Res. Lett., 39, L23706.

Barnett, T. P., D. W. Pierce, and R. Schnur, 2001: Detection of anthropogenic climate change in the world's oceans. Science, 292, 270-274.

Barnett, T. P., D. W. Pierce, K. Achutarao, P. Gleckler, B. Santer, J. Gregory, and W. Washington, 2005: Penetration of human-induced warming into the world's oceans. Science, 309, 284-287.

Barnett, T. P., et al., 2008: Human-induced changes in the hydrology of the western United States. Science, 319, 1080-1083.

Barriopedro, D., R. Garcia-Herrera, and R. Huth, 2008: Solar modulation of Northern Hemisphere blocking. J. Geophys. Res. Atmos., 113, D14118.

Beenstock, M., Y. Reingewertz, and N. Paldor, 2012: Polynomial cointegration tests of anthropogenic impact on global warming. Earth Syst. Dyn. Discuss., 3, 561-596.

Bender, F. A. M., A. M. L. Ekman, and H. Rodhe, 2010: Response to the eruption of Mount Pinatubo in relation to climate sensitivity in the CMIP3 models. Clim. Dyn., 35, 875-886.

Benestad, R. E., and G. A. Schmidt, 2009: Solar trends and global warming. J. Geophys. Res. Atmos., 114, D14101.

Bengtsson, L., and K. I. Hodges, 2011: On the evaluation of temperature trends in the tropical troposphere. Clim. Dyn., 36 419-430.

Bengtsson, L., V. A. Semenov, and O. M. Johannessen, 2004: The early twentiethcentury warming in the Arctic-A possible mechanism. J. Clim., 17, 4045-4057.

Bengtsson, L., K. I. Hodges, E. Roeckner, and R. Brokopf, 2006: On the natural variability of the pre-industrial European climate. Clim. Dyn., 27, 743-760.

Berliner, L. M., A. L. Richard, and J. S. Dennis, 2000: Bayesian climate change assessment. J. Clim., 13, 3805-3820.

Bhend, J., and H. von Storch, 2008: Consistency of observed winter precipitation trends in northern Europe with regional climate change projections. Clim. Dyn., $31,17-28$.

Bindoff, N. L., and T. J. McDougall, 2000: Decadal changes along an Indian ocean section at 32 degrees $S$ and their interpretation. J. Phys. Oceanogr., 30, 12071222.

Bindoff, N. L., et al., 2007: Observations: Oceanic climate change and sea level. In: Climate Change 2007: The Physical Science Basis. Contribution of Working Group I to the Fourth Assessment Report of the Intergovernmental Panel on Climate Change [Solomon, S., D. Qin, M. Manning, Z. Chen, M. Marquis, K. B. Averyt, M. Tignor and H. L. Miller (eds.)] Cambridge University Press, Cambridge, United Kingdom and New York, NY, USA, pp. 385-432.

Bintanja, R., G. J. van Oldenborgh, S. S. Drijfhout, B. Wouters, and C. A. Katsman, 2013: Important role for ocean warming and increased ice-shelf melt in Antarctic sea-ice expansion. Nature Geosci., 6, 376-379.

Birner, T., 2010: Recent widening of the tropical belt from global tropopause statistics: Sensitivities. J. Geophys. Res. Atmos., 115, D23109.

Bitz, C. M., and L. M. Polvani, 2012: Antarctic climate response to stratospheric ozone depletion in a fine resolution ocean climate model. Geophys. Res. Lett., 39, L20705.

Boer, G. J., 2011: The ratio of land to ocean temperature change under global warming Clim. Dyn., 37, 2253-2270.

Boer, G. J., M. Stowasser, and K. Hamilton, 2007: Inferring climate sensitivity from volcanic events. Clim. Dyn., 28, 481-502.

Bonfils, C., P. B. Duffy, B. D. Santer, T. M. L. Wigley, D. B. Lobell, T. J. Phillips, and C. Doutriaux, 2008: Identification of external influences on temperatures in California. Clim. Change, 87, S43-S55.

Booth, B. B. B., N. J. Dunstone, P. R. Halloran, T. Andrews, and N. Bellouin, 2012 Aerosols implicated as a prime driver of twentieth-century North Atlantic climate variability. Nature, 484, 228-232.

Bowerman, N. H. A., D. J. Frame, C. Huntingford, J. A. Lowe, and M. R. Allen, 2011: Cumulative carbon emissions, emissions floors and short-term rates of warming: Implications for policy. Philos. Trans. R. Soc. A, 369, 45-66.

Boyer, T. P., S. Levitus, J. I. Antonov, R. A. Locarnini, and H. E. Garcia, 2005: Linear trends in salinity for the World Ocean, 1955-1998. Geophys. Res. Lett., 32, L01604. 
Brandt, P., et al., 2010: Changes in the ventilation of the oxygen minimum zone of the tropical North Atlantic. J. Phys. Oceanogr., 40, 1784-1801.

Brayshaw, D. J., B. Hoskins, and M. Blackburn, 2008: The storm-track response to idealized SST perturbations in an aquaplanet GCM. J. Atmos. Sci., 65, 28422860.

Brohan, P., J. J. Kennedy, I. Harris, S. F. B. Tett, and P. D. Jones, 2006: Uncertainty estimates in regional and global observed temperature changes: A new data set from 1850. J. Geophys. Res. Atmos., 111, D12106.

Bromwich, D. H., J. P. Nicolas, A. J. Monaghan, M. A. Lazzara, L. M. Keller, G. A. Weidner, and A. B. Wilson, 2013: Central West Antarctica among the most rapidly warming regions on Earth. Nature Geosci., 6, 139-145.

Brönnimann, S., 2009: Early twentieth-century warming. Nature Geosci, 2, 735-736.

Brönnimann, S., et al., 2012: A multi-data set comparison of the vertical structure of temperature variability and change over the Arctic during the past 100 year. Clim. Dyn., 39 1577-1598.

Brown, R. D., and D. A. Robinson, 2011: Northern Hemisphere spring snow cover variability and change over 1922-2010 including an assessment of uncertainty. Cryosphere, 5, 219-229.

Burke, E. J., S. J. Brown, and N. Christidis, 2006: Modeling the recent evolution of global drought and projections for the twenty-first century with the Hadley Centre climate model. J. Hydrometeorol., 7 1113-1125.

Butchart, N., et al., 2011: Multimodel climate and variability of the stratosphere. J. Geophys. Res. Atmos., 116, D05102.

Butler, A. H., D. W. Thompson, and R. Heikes, 2010: The steady-state atmospheric circulation response to climate change-like thermal forcings in a simple general circulation model. J. Clim., 23, 3474-3496.

Cai, M., and K.-K. Tung, 2012: Robustness of dynamical feedbacks from radiative forcing: $2 \%$ solar versus 2 XCO2 experiments in an idealized GCM. J. Atmos. Sci, 69, 2256-2271.

Cattiaux, J., R. Vautard, C. Cassou, P. Yiou, V. Masson-Delmotte, and F. Codron, 2010: Winter 2010 in Europe: A cold extreme in a warming climate. Geophys. Res. Lett., 37, L20704.

Cayan, D. R., T. Das, D. W. Pierce, T. P. Barnett, M. Tyree, and A. Gershunov, 2010: Future dryness in the southwest US and the hydrology of the early 21st century drought. Proc. Natl. Acad. Sci. U.S.A., 107, 21271-21276.

Charlton-Perez, A. J., et al., 2013: On the lack of stratospheric dynamical variability in low-top versions of the CMIP5 models. J. Geophys. Res. Atmos., 118, 2494-2505.

Chinn, T., S. Winkler, M. J. Salinger, and N. Haakensen, 2005: Recent glacier advances in Norway and New Zealand: A comparison of their glaciological and meteorological causes. Geograf. Annal. A, 87, 141-157.

Christiansen, B., and F. C. Ljungqvist, 2011: Reconstruction of the extratropical NH mean temperature over the last millennium with a method that preserves lowfrequency variability. J. Clim., 24, 6013-6034.

Christidis, N., P. A. Stott, and S. J. Brown, 2011: The role of human activity in the recent warming of extremely warm daytime temperatures. J. Clim., 24, 1922-1930.

Christidis, N., P. A. Stott, G. C. Hegerl, and R. A. Betts, 2013: The role of land use change in the recent warming of daily extreme temperatures. Geophys. Res. Lett., 40, 589-594.

Christidis, N., P. A. Stott, F. W. Zwiers, H. Shiogama, and T. Nozawa, 2010: Probabilistic estimates of recent changes in temperature: A multi-scale attribution analysis. Clim. Dyn., 34, 1139-1156.

Christidis, N., P. A. Stott, F. W. Zwiers, H. Shiogama, and T. Nozawa, 2012a: The contribution of anthropogenic forcings to regional changes in temperature during the last decade. Clim. Dyn., 39, 1259-1274.

Christidis, N., P. A. Stott, G. S. Jones, H. Shiogama, T. Nozawa, and J. Luterbacher, 2012b: Human activity and anomalously warm seasons in Europe. Int. J. Climatol., 32, 225-239.

Chung, E. S., B. J. Soden, and B. J. Sohn, 2010: Revisiting the determination of climate sensitivity from relationships between surface temperature and radiative fluxes. Geophys. Res. Lett., 37, L10703.

Church, J., N. White, and J. Arblaster, 2005: Significant decadal-scale impact of volcanic eruptions on sea level and ocean heat content. Nature, 438, 74-77.

Church, J. A., D. Monselesan, J. M. Gregory, and B. Marzeion, 2013: Evaluating the ability of process based models to project sealevel change. Environ. Res. Lett., 8, 014051.

Church, J. A., et al., 2011: Revisiting the Earth's sea-level and energy budgets from 1961 to 2008. Geophys. Res. Lett., 38, L18601.
Chylek, P., and U. Lohmann, 2008a: Reply to comment by Andrey Ganopolski and Thomas Schneider von Deimling on "Aerosol radiative forcing and climate sensitivity deduced from the Last Glacial Maximum to Holocene transition". Geophys. Res. Lett., 35, L23704.

Chylek, P., and U. Lohmann, 2008b: Aerosol radiative forcing and climate sensitivity deduced from the last glacial maximum to Holocene transition. Geophys. Res. Lett., 35, L04804.

Chylek, P., U. Lohmann, M. Dubey, M. Mishchenko, R. Kahn, and A. Ohmura, 2007: Limits on climate sensitivity derived from recent satellite and surface observations. J. Geophys. Res. Atmos., 112, D24S04.

Comiso, J. C., 2012: Large decadal decline in Arctic multiyear ice cover. J. Clim., 25, 1176-1193.

Comiso, J. C., and F. Nishio, 2008: Trends in the sea ice cover using enhanced and compatible AMSR-E, SSM/I, and SMMR data. J. Geophys. Res. Oceans, 113, C02S07.

Compo, G. P., et al., 2011: The twentieth century reanalysis project. Q. J. R. Meteorol. Soc., 137, 1-28.

Cordero, E. C., and P. M. D. Forster, 2006: Stratospheric variability and trends in models used for the IPCC AR4. Atmos. Chem. Phys., 6, 5369-5380.

Crook, J. A., and P. M. Forster, 2011:A balance between radiative forcing and climate feedback in the modeled 20th century temperature response. J. Geophys. Res. Atmos., 116, D17108.

Crook, J. A., P. M. Forster, and N. Stuber, 2011: Spatial patterns of modeled climate feedback and contributions to temperature response and polar amplification. $J$. Clim., 24, 3575-3592.

Curry, J. A., and P. J. Webster, 2011: Climate science and the uncertainty monster. Bull. Am. Meteorol. Soc., 92, 1667-1682.

Curry, R., B. Dickson, and I. Yashayaev, 2003: A change in the freshwater balance of the Atlantic Ocean over the past four decades. Nature, 426, 826-829

D'Arrigo, R., R. Wilson, and G. Jacoby, 2006: On the long-term context for late twentieth century warming. J. Geophys. Res. Atmos., 111 D03103.

D'Arrigo, R., R. Wilson, and A. Tudhope, 2009: The impact of volcanic forcing on tropical temperatures during the past four centuries. Nature Geosci., 2, 51-56.

Dai, A., 2011: Drought under global warming: A review. WIREs Clim. Change, 2, 45-65.

Dai, A., 2013: Increasing drought under global warming in observations and models. Nature Clim. Change, 3, 52-58.

Dall'Amico, M., L. J. Gray, K. H. Rosenlof, A. A. Scaife, K. P. Shine, and P. A. Stott, 2010: Stratospheric temperature trends: Impact of ozone variability and the QBO. Clim. Dyn., 34, 381-398.

Davis, S. M., and K. H. Rosenlof, 2012: A multidiagnostic intercomparison of tropicalwidth time series using reanalyses and satellite observations. J. Clim., 25, 10611078.

Day, J. J., J. C. Hargreaves, J. D. Annan, and A. Abe-Ouchi, 2012: Sources of multidecadal variability in Arctic sea ice extent. Environ. Res. Lett., 7, 034011.

Dean, S. M., and P. A. Stott, 2009: The effect of local circulation variability on the detection and attribution of New Zealand temperature trends. J. Clim., 22, 6217-6229.

DelSole, T., M. K. Tippett, and J. Shukla, 2011: A significant component of unforced multidecadal variability in the recent acceleration of global warming. J. Clim. 24, 909-926.

Delworth, T., V. Ramaswamy, and G. Stenchikov, 2005: The impact of aerosols on simulated ocean temperature and heat content in the 20th century. Geophys. Res. Lett., 32, L24709.

Delworth, T. L., and M. E. Mann, 2000: Observed and simulated multidecadal variability in the Northern Hemisphere. Clim. Dyn., 16, 661-676.

Deser, C., and H. Teng, 2008: Evolution of Arctic sea ice concentration trends and the role of atmospheric circulation forcing, 1979-2007. Geophys. Res. Lett., 35, L02504.

Dessler, A. E., 2010: A determination of the cloud feedback from climate variations over the past decade. Science, 330, 1523-1527.

Dessler, A. E., 2011: Cloud variations and the Earth's energy budget. Geophys. Res. Lett., 38, L19701.

Dickson, R. R., et al., 2000: The Arctic Ocean response to the North Atlantic oscillation. J. Clim., 13, 2671-2696.

Ding, Q. H., E. J. Steig, D. S. Battisti, and M. Kuttel, 2011: Winter warming in West Antarctica caused by central tropical Pacific warming. Nature Geosci., 4, 398403. 
Doherty, S. J., et al., 2009: Lessons learned from IPCC AR4 scientific developments needed to understand, predict, and respond to climate change. Bull. Am. Meteorol. Soc., 90, 497-513.

Dole, R., et al., 2011: Was there a basis for anticipating the 2010 Russian heat wave? Geophys. Res. Lett., 38, L06702.

Domingues, C., J. Church, N. White, P. Gleckler, S. Wijffels, P. Barker, and J. Dunn, 2008: Improved estimates of upper-ocean warming and multi-decadal sea-level rise. Nature, 453, 1090-1093.

Doscher, R., K. Wyser, H. E. M. Meier, M. W. Qian, and R. Redler, 2010: Quantifying Arctic contributions to climate predictability in a regional coupled ocean-iceatmosphere model. Clim. Dyn., 34, 1157-1176.

Douglass, D. H., E. G. Blackman, and R. S. Knox, 2004: Corrigendum to: Temperature response of Earth to the annual solar irradiance cycle [Phys. Lett. A 323 (2004) 315]. Phys. Lett. A, 325, 175-176.

Douville, H., A. Ribes, B. Decharme, R. Alkama, and J. Sheffield, 2013: Anthropogenic influence on multidecadal changes in reconstructed global evapotranspiration. Nature Clim. Change, 3, 59-62.

Driscoll, S., A. Bozzo, L. J. Gray, A. Robock, and G. Stenchikov, 2012: Coupled Model Intercomparison Project 5 (CMIP5) simulations of climate following volcanic eruptions. J. Geophys. Res., 117, D17105.

Drost, F., and D. Karoly, 2012 Evaluating global climate responses to different forcings using simple indices. Geophys. Res. Lett., 39, L16701.

Drost, F., D. Karoly, and K. Braganza, 2012: Communicating global climate change using simple indices: An update Clim. Dyn., 39, 989-999.

Duarte, C. M., T. M. Lenton, P. Wadhams, and P. Wassmann, 2012: Abrupt climate change in the Arctic. Nature Clim. Change, 2, 60-62.

Durack, P., S. Wijffels, and R. Matear, 2012: Ocean salinities reveal strong global water cycle intensification during 1950 to 2000 . Science, 336, 455-458.

Durack, P. J., and S. E. Wijffels, 2010: Fifty-year trends in global ocean salinities and their relationship to broad-scale warming. J. Clim., 23, 4342-4362.

Edwards, T. L., M. Crucifix, and S. P. Harrison, 2007: Using the past to constrain the future: How the palaeorecord can improve estimates of global warming. Prog. Phys. Geogr., 31, 481-500.

Elsner, J. B., 2006: Evidence in support of the climate change-Atlantic hurricane hypothesis. Geophys. Res. Lett., 33, L16705.

Elsner, J. B., J. P. Kossin, and T. H. Jagger, 2008: The increasing intensity of the strongest tropical cyclones. Nature, 455, 92-95.

Emanuel, K., 2005: Increasing destructiveness of tropical cyclones over the past 30 years. Nature, 436, 686-688.

Emanuel, K., S. Solomon, D. Folini, S. Davis, and C. Cagnazzo, 2013: Influence of tropical tropopause layer cooling on Atlantic hurricane activity. J. Clim., 26, 2288-2301.

Emerson, S., Y. W. Watanabe, T. Ono, and S. Mecking, 2004: Temporal trends in apparent oxygen utilization in the upper pycnocline of the North Pacific: 19802000. J. Oceanogr., 60, 139-147.

Engle, R. F., and C. W. J. Granger, 1987: Co-integration and error correction: Representation, estimation, and testing. Econometrica, 55, 251-276.

Esper, J., et al., 2012: Orbital forcing of tree-ring data. Nature Clim. Change, 2, 862-866.

Evan, A. T., G. R. Foltz, D. X. Zhang, and D. J. Vimont, 2011: Influence of African dust on ocean-atmosphere variability in the tropical Atlantic. Nature Geosci., 4, 762-765.

Evan, A. T., D. J. Vimont, A. K. Heidinger, J. P. Kossin, and R. Bennartz, 2009: The role of aerosols in the evolution of tropical North Atlantic Ocean temperature anomalies. Science, 324, 778-781.

Eyring, V., et al., 2013: Long-term changes in tropospheric and stratospheric ozone and associated climate impacts in CMIP5 simulations. J. Geophys. Res. Atmos., doi:10.1002/jgrd.50316.

Eyring, V., et al., 2006: Assessment of temperature, trace species, and ozone in chemistry-climate model simulations of the recent past. J. Geophys. Res. Atmos., 111, D22308.

Fernández-Donado, L., et al., 2013: Large-scale temperature response to external forcing in simulations and reconstructions of the last millennium. Clim. Past, 9, 393-421.

Fettweis, X., G. Mabille, M. Erpicum, S. Nicolay, and M. Van den Broeke, 2011: The 1958-2009 Greenland ice sheet surface melt and the mid-tropospheric atmospheric circulation. Clim. Dyn., 36, 139-159.
Fettweis, X., B. Franco, M. Tedesco, J. H. van Angelen, J. T. M. Lenaerts, M. R. van den Broeke, and H. Gallée, 2013: Estimating the Greenland ice sheet surface mass balance contribution to future sea level rise using the regional atmospheric climate model MAR. Cryosphere, 7, 469-489.

Feulner, G., 2011: Are the most recent estimates for Maunder Minimum solar irradiance in agreement with temperature reconstructions? Geophys. Res. Lett., 38, L16706.

Fischer, E. M., S. I. Seneviratne, P. L. Vidale, D. Luthi, and C. Schar, 2007: Soil moisture -atmosphere interactions during the 2003 European summer heat wave. $J$. Clim., 20, 5081-5099.

Fogt, R. L., J. Perlwitz, A. J. Monaghan, D. H. Bromwich, J. M. Jones, and G. J. Marshall, 2009: Historical SAM variability. Part II: Twentieth-century variability and trends from reconstructions, observations, and the IPCC AR4 models. J. Clim., 22, 5346-5365.

Folland, C. K., et al., 2013 High predictive skill of global surface temperature a year ahead. Geophys. Res. Lett., 40, 761-767.

Forest, C. E., and R. W. Reynolds, 2008: Climate change-Hot questions of temperature bias. Nature, 453, 601-602.

Forest, C. E., P. H. Stone, and A. P. Sokolov, 2006: Estimated PDFs of climate system properties including natural and anthropogenic forcings. Geophys. Res. Lett., 33, L01705.

Forest, C. E., P. H. Stone, and A. P. Sokolov, 2008: Constraining climate model parameters from observed 20th century changes. Tellus A, 60, 911-920.

Forest, C. E., M. R. Allen, P. H. Stone, and A. P. Sokolov, 2000: Constraining uncertainties in climate models using climate change detection techniques. Geophys. Res. Lett., 27, 569-572.

Forest, C. E., P. H. Stone, A. P. Sokolov, M. R. Allen, and M. D. Webster, 2002: Quantifying uncertainties in climate system properties with the use of recent climate observations. Science, 295, 113-117.

Forster, P., et al., 2007: Changes in atmospheric constituents and in radiative forcing. In: Climate Change 2007: The Physical Science Basis. Contribution of Working Group I to the Fourth Assessment Report of the Intergovernmental Panel on Climate Change [Solomon, S., D. Qin, M. Manning, Z. Chen, M. Marquis, K. B. Averyt, M. Tignor and H. L. Miller (eds.)] Cambridge University Press, Cambridge, United Kingdom and New York, NY, USA, pp. 129-234.

Forster, P. M., T. Andrews, P. Good, J. M. Gregory, L. S. Jackson, and M. Zelinka, 2013 Evaluating adjusted forcing and model spread for historical and future scenarios in the CMIP5 generation of climate models. J. Geophys. Res. Atmos., 118, 11391150.

Forster, P. M., et al., 2011: Stratospheric changes and climate. In: Scientific Assessment of Ozone Depletion: 2010. Global Ozone Research and Monitoring ProjectReport No. 52 [P. M. Forster and D. W. J. Thompson (eds.)]. World Meteorological Organization, Geneva, Switzerland, $516 \mathrm{pp}$.

Forster, P. M. D., and J. M. Gregory, 2006: The climate sensitivity and its components diagnosed from Earth Radiation Budget data. J. Clim., 19, 39-52.

Foster, G., and S. Rahmstorf, 2011: Global temperature evolution 1979-2010. Environ. Res. Lett., 6, 044022.

Foster, G., J. D. Annan, G. A. Schmidt, and M. E. Mann, 2008: Comment on "Heat capacity, time constant, and sensitivity of Earth's climate system" by S. E. Schwartz. J. Geophys. Res. Atmos., 113, D15102.

Fowler, H. J., and R. L. Wilby, 2010: Detecting changes in seasonal precipitation extremes using regional climate model projections: Implications for managing fluvial flood risk. Water Resour. Res., 46, W03525.

Frame, D. J., D. A. Stone, P. A. Stott, and M. R. Allen, 2006: Alternatives to stabilization scenarios. Geophys. Res. Lett., 33, L14707.

Frame, D. J., B. B. B. Booth, J. A. Kettleborough, D. A. Stainforth, J. M. Gregory, M. Collins, and M. R. Allen, 2005: Constraining climate forecasts: The role of prior assumptions. Geophys. Res. Lett., 32, L09702.

Francis, J. A., and S. J. Vavrus 2012: Evidence linking Arctic amplification to extreme weather in mid-latitudes. Geophys. Res. Lett., 39, L06801.

Frank, D., J. Esper, and E. R. Cook, 2007: Adjustment for proxy number and coherence in a large-scale temperature reconstruction. Geophys. Res. Lett., 34, L16709.

Frank, D. C., J. Esper, C. C. Raible, U. Buntgen, V. Trouet, B. Stocker, and F. Joos, 2010: Ensemble reconstruction constraints on the global carbon cycle sensitivity to climate. Nature, 463, 527-530.

Franzke, C., 2010: Long-range dependence and climate noise characteristics of Antarctic temperature data. J. Clim., 23, 6074-6081.

Free, M., 2011: The seasonal structure of temperature trends in the tropical lower stratosphere. J. Clim., 24, 859-866. 
Free, M., and J. Lanzante, 2009: Effect of volcanic eruptions on the vertical temperature profile in radiosonde data and climate models. J. Clim., 22, 29252939.

Friedlingstein, P., et al., 2006: Climate-carbon cycle feedback analysis: Results from the C4MIP model intercomparison. J. Clim., 19, 3337-3353.

Frierson, D. M. W., 2006: Robust increases in midlatitude static stability in simulations of global warming. Geophys. Res. Lett., 33, L24816.

Frierson, D. M. W., J. Lu, and G. Chen, 2007: Width of the Hadley cell in simple and comprehensive general circulation models. Geophys. Res. Lett., 34, L18804.

Fu, Q., and P. Lin, 2011: Poleward shift of subtropical jets inferred from satelliteobserved lower stratospheric temperatures. J. Clim., 24, 5597-5603.

Fu, Q., S. Solomon, and P. Lin, 2010: On the seasonal dependence of tropical lowerstratospheric temperature trends. Atmos. Chem. Phys., 10, 2643-2653.

Fu, Q., S. Manabe, and C. M. Johanson, 2011: On the warming in the tropical upper troposphere: Models versus observations. Geophys. Res. Lett., 38, L15704.

Fu, Q., C. M. Johanson, J. M. Wallace, and T. Reichler, 2006: Enhanced mid-latitude tropospheric warming in satellite measurements. Science, 312, 1179-1179.

Fyfe, J. C., 2006: Southern Ocean warming due to human influence. Geophys. Res. Lett., 33, L19701.

Fyfe, J. C., N. P. Gillett, and D. W. J. Thompson, 2010: Comparing variability and trends in observed and modelled global-mean surface temperature. Geophys. Res. Lett., 37, L16802.

Fyfe, J. C., N. P. Gillett, and G. J. Marshal, 2012: Human influence on extratropical Southern Hemisphere summer precipitation. Geophys. Res. Lett., 39, L23711.

Fyke, J., and M. Eby, 2012: Comment on "Climate sensitivity estimated from temperature reconstructions of the Last Glacial Maximum". Science, 337, 1294.

Ganopolski, A., and T. S. von Deimling, 2008: Comment on "Aerosol radiative forcing and climate sensitivity deduced from the Last Glacial Maximum to Holocene transition" by Petr Chylek and Ulrike Lohmann. Geophys. Res. Lett., 35, L23703.

Gascard, J. C., et al., 2008: Exploring Arctic transpolar drift during dramatic sea ice retreat. Eos Trans. Am. Geophys. Union, 89, 21-22.

Gay-Garcia, C., F. Estrada, and A. Sanchez, 2009: Global and hemispheric temperature revisited. Clim. Change, 94, 333-349

Giles, K. A., S. W. Laxon, and A. L. Ridout, 2008: Circumpolar thinning of Arctic sea ice following the 2007 record ice extent minimum. Geophys. Res. Lett., 35, L22502.

Gillett, N. P., and P. A. Stott, 2009: Attribution of anthropogenic influence on seasonal sea level pressure. Geophys. Res. Lett., 36, L23709.

Gillett, N. A., and J. C. Fyfe, 2013: Annular Mode change in the CMIP5 simulations. Geophys. Res. Lett., 40, 1189-1193.

Gillett, N. P., R. J. Allan, and T. J. Ansell, 2005: Detection of external influence on sea level pressure with a multi-model ensemble. Geophys. Res. Lett., 32, L19714.

Gillett, N. P., P. A. Stott, and B. D. Santer, 2008a: Attribution of cyclogenesis region sea surface temperature change to anthropogenic influence. Geophys. Res. Lett., 35, L09707.

Gillett, N. P., G. C. Hegerl, M. R. Allen, and P. A. Stott, 2000: Implications of changes in the Northern Hemisphere circulation for the detection of anthropogenic climate change. Geophys. Res. Lett., 27, 993-996.

Gillett, N. P., F. W. Zwiers, A. J. Weaver, and P. A. Stott, 2003: Detection of human influence on sea-level pressure. Nature, 422, 292-294.

Gillett, N. P., M. F. Wehner, S. F. B. Tett, and A. J. Weaver, 2004: Testing the linearity of the response to combined greenhouse and sulfate aerosol forcing. Geophys. Res. Lett., 31, L14201.

Gillett, N. P., V. K. Arora, G. M. Flato, J. F. Scinocca, and K. von Salzen, 2012: Improved constraints on 21st-century warming derived using 160 years of temperature observations. Geophys. Res. Lett., 39, L01704.

Gillett, N. P., V. K. Arora, D. Matthews, P. A. Stott, and M. R. Allen, 2013 Constraining the ratio of global warming to cumulative $\mathrm{CO}_{2}$ emissions using CMIP5 simulations. J. Clim., doi:10.1175/JCLI-D-12-00476.1.

Gillett, N. P., et al., 2008b: Attribution of polar warming to human influence. Nature Geosci., 1, 750-754.

Gillett, N. P., et al., 2011: Attribution of observed changes in stratospheric ozone and temperature. Atmos. Chem. Phys., 11 599-609.

Gleckler, P. J., T. M. L. Wigley, B. D. Santer, J. M. Gregory, K. AchutaRao, and K. E. Taylor, 2006: Volcanoes and climate: Krakatoa's signature persists in the ocean. Nature, 439, 675-675.

Gleckler, P. J., et al., 2012: Human-induced global ocean warming on multidecadal timescales. Nature Clim. Change, 2, 524-529.

Gong, D., and S. Wang, 1999: Definition of Antarctic oscillation index. Geophys. Res. Lett., 26, 459-462.
Goosse, H., W. Lefebvre, A. de Montety, E. Crespin, and A. H. Orsi, 2009: Consistent past half-century trends in the atmosphere, the sea ice and the ocean at high southern latitudes. Clim. Dyn., 33, 999-1016.

Goosse, H., J. Guiot, M. E. Mann, S. Dubinkina, and Y. Sallaz-Damaz, 2012a: The medieval climate anomaly in Europe: Comparison of the summer and annual mean signals in two reconstructions and in simulations with data assimilation. Global Planet. Change, 84-85, 35-47.

Goosse, H., E. Crespin, A. de Montety, M. E. Mann, H. Renssen, and A. Timmermann, 2010: Reconstructing surface temperature changes over the past 600 years using climate model simulations with data assimilation. J. Geophys. Res. Atmos. 115, D09108.

Goosse, H., et al., 2012b: The role of forcing and internal dynamics in explaining the "Medieval Climate Anomaly'. Clim. Dyn., 39, 2847-2866.

Gouretski, V., and K. Koltermann, 2007: How much is the ocean really warming? Geophys. Res. Lett., 34, L01610.

Graff, L. S., and J. H. LaCasce, 2012: Changes in the extratropical storm tracks in response to changes in SST in an GCM. J. Clim., 25, 1854-1870.

Grant, A. N., S. Bronnimann, T. Ewen, T. Griesser, and A. Stickler, 2009: The early twentieth century warm period in the European Arctic. Meteorol. Z., 18 425432.

Graversen, R. G., and M. H. Wang, 2009: Polar amplification in a coupled climate model with locked albedo. Clim. Dyn., 33, 629-643.

Gray, L. J., et al., 2010: Solar influences on climate. Rev. Geophys. 48 RG4001.

Gregory, J. M., 2000: Vertical heat transports in the ocean and their effect an timedependent climate change. Clim. Dyn., 16, 501-515.

Gregory, J. M., 2010: Long-term effect of volcanic forcing on ocean heat content. Geophys. Res. Lett., 37, L22701.

Gregory, J. M., and P. M. Forster, 2008: Transient climate response estimated from radiative forcing and observed temperature change. J. Geophys. Res. Atmos., 113, D23105.

Gregory, J. M., J. A. Lowe, and S. F. B. Tett, 2006: Simulated global-mean sea level changes over the last half-millennium. J. Clim., 19, 4576-4591.

Gregory, J. M., C. D. Jones, P. Cadule, and P. Friedlingstein, 2009: Quantifying carbon cycle feedbacks. J. Clim., 22, 5232-5250.

Gregory, J. M., R. J. Stouffer, S. C. B. Raper, P. A. Stott, and N. A. Rayner, 2002: An observationally based estimate of the climate sensitivity. J. Clim., 15, 31173121.

Gregory, J. M., H. T. Banks, P. A. Stott, J. A. Lowe, and M. D. Palmer, 2004: Simulated and observed decadal variability in ocean heat content. Geophys. Res. Lett., 31 L15312.

Gregory, J. M., et al., 2012 Twentieth-century global-mean sea-level rise: Is the whole greater than the sum of the parts? J. Clim., doi:10.1175/JCLI-D-12-00319.1.

Grist, J., et al., 2010: The roles of surface heat flux and ocean heat transport convergence in determining Atlantic Ocean temperature variability. Ocean Dyn., $60,771-790$.

Haam, E., and K. K. Tung, 2012: Statistics of solar cycle-La Niña connection: Correlation of two autocorrelated time series. J. Atmos. Sci., 69 2934-2939.

Haigh, J., M. Blackburn, and R. Day, 2005: The response of tropospheric circulation to perturbations in lower-stratospheric temperature. J. Clim., 18, 3672-3685.

Haigh, J. D., 1996: The impact of solar variability on climate. Science, 272 981-984.

Haimberger, L., C. Tavolato, and S. Sperka, 2012: Homogenization of the global radiosonde temperature dataset through combined comparison with reanalysis background series and neighboring stations. J. Clim., 25, 8108-8131.

Han, W., et al., 2010: Patterns of Indian Ocean sea-level change in a warming climate. Nature Geosci, 3, 546-550.

Hanna, E., J. M. Jones, J. Cappelen, S. H. Mernild, L. Wood, K. Steffen, and P. Huybrechts, 2013: The influence of North Atlantic atmospheric and oceanic forcing effects on 1900-2010 Greenland summer climate and ice melt/runoff. Int. J. Climatol., 33, 862-880.

Hanna, E., et al., 2008: Increased runoff from melt from the Greenland ice sheet: A response to global warming. J. Clim., 21, 331-341.

Hannart, A., J. L. Dufresne, and P. Naveau, 2009: Why climate sensitivity may not be so unpredictable. Geophys. Res. Lett., 36, L16707.

Hansen, J., and S. Lebedeff, 1987: Global trends of measured surface air-temperature. J. Geophys. Res. Atmos., 92, 13345-13372.

Hansen, J., M. Sato, and R. Ruedy, 2012: Perception of climate change. Proc. Natl. Acad. Sci. U.S.A., 109, 14726-14727.

Hansen, J., M. Sato, P. Kharecha, and K. von Schuckmann, 2011: Earth's energy imbalance and implications. Atmos. Chem. Phys., 11, 13421-13449. 
Hansen, J., et al., 2005a: Earth's energy imbalance: Confirmation and implications. Science, 308, 1431-1435.

Hansen, J., et al., 2005b: Efficacy of climate forcings. J. Geophys. Res. Atmos., 110, D18104.

Hargreaves, J. C., and J. D. Annan, 2009: Comment on 'Aerosol radiative forcing and climate sensitivity deduced from the Last Glacial Maximum to Holocene transition', by P. Chylek and U. Lohmann, Geophys. Res. Lett., doi:10.1029/2007GL032759., 2008. Clim. Past, 5, 143-145.

Hargreaves, J. C., A. Abe-Ouchi, and J. D. Annan, 2007: Linking glacial and future climates through an ensemble of GCM simulations. Clim. Past, 3, 77-87.

Hargreaves, J. C., J. D. Annan, M. Yoshimori, and A. Abe-Ouchi, 2012: Can the Last Glacial Maximum constrain climate sensitivity? Geophys. Res. Lett., 39, L24702.

Harris, G. R., D. M. H. Sexton, B. B. B. Booth, M. Collins, and J. M. Murphy, 2013: Probabilistic projections of transient climate change. Clim. Dyn., doi:10.1007/ s00382-012-1647-y.

Hasselmann, K., 1997: Multi-pattern fingerprint method for detection and attribution of climate change. Clim. Dyn., 13, 601-611.

Hegerl, G., and F. Zwiers, 2011: Use of models in detection and attribution of climate change. WIREs Clim. Change, 2, 570-591.

Hegerl, G., J. Luterbacher, F. Gonzalez-Rouco, S. F. B. Tett, T. Crowley, and E. Xoplaki, 2011a: Influence of human and natural forcing on European seasonal temperatures. Nature Geosci., 4, 99-103.

Hegerl, G. C., F. W. Zwiers, and C. Tebaldi, 2011b: Patterns of change: Whose fingerprint is seen in global warming? Environ. Res. Lett., 6, 044025.

Hegerl, G. C., F. W. Zwiers, P. A. Stott, and V. V. Kharin, 2004: Detectability of anthropogenic changes in annual temperature and precipitation extremes. $J$. Clim., 17, 3683-3700.

Hegerl, G. C., T. J. Crowley, W. T. Hyde, and D. J. Frame, 2006: Climate sensitivity constrained by temperature reconstructions over the past seven centuries. Nature, 440, 1029-1032.

Hegerl, G. C., P. Stott, S. Solomon, and F. W. Zwiers, 2011c: Comment on "Climate science and the uncertainty monster by J.A. Curry and P.J. Webster". Bull. Am. Meteorol. Soc., 92, 1683-1685.

Hegerl, G. C., T. J. Crowley, S. K. Baum, K. Y. Kim, and W. T. Hyde, 2003: Detection of volcanic, solar and greenhouse gas signals in paleo-reconstructions of Northern Hemispheric temperature. Geophys. Res. Lett., 30, 1242.

Hegerl, G. C., T. J. Crowley, M. Allen, W. T. Hyde, H. N. Pollack, J. Smerdon, and E. Zorita, 2007a: Detection of human influence on a new, validated 1500-year temperature reconstruction. J. Clim., 20, 650-666.

Hegerl, G. C., et al., 2010: Good practice guidance paper on detection and attribution related to anthropogenic climate change. In: Meeting Report of the Intergovernmental Panel on Climate Change Expert Meeting on Detection and Attribution of Anthropogenic Climate Change [T. F. Stocker, et al. (eds.)]. IPCC Working Group I Technical Support Unit, University of Bern, Bern, Switzerland, $8 \mathrm{pp}$.

Hegerl, G. C., et al., 2007b: Understanding and attributing climate change. In: Climate Change 2007: The Physical Science Basis. Contribution of Working Group I to the Fourth Assessment Report of the Intergovernmental Panel on Climate Change [Solomon, S., D. Qin, M. Manning, Z. Chen, M. Marquis, K. B. Averyt, M. Tignor and H. L. Miller (eds.)] Cambridge University Press, Cambridge, United Kingdom and New York, NY, USA, pp. 663-745.

Held, I. M., and B. J. Soden, 2006: Robust responses of the hydrological cycle to global warming. J. Clim., 19, 5686-5699.

Held, I. M., M. Winton, K. Takahashi, T. Delworth, F. R. Zeng, and G. K. Vallis, 2010: Probing the fast and slow components of global warming by returning abruptly to preindustrial forcing. J. Clim., 23, 2418-2427.

Helm, K. P., N. L. Bindoff, and J. A. Church, 2010: Changes in the global hydrologicalcycle inferred from ocean salinity. Geophys. Res. Lett., 37, L18701.

Helm, K. P., N. L. Bindoff, and J. A. Church, 2011: Observed decreases in oxygen content of the global ocean. Geophys. Res. Lett., 38, L23602.

Henriksson, S. V., E. Arjas, M. Laine, J. Tamminen, and A. Laaksonen, 2010: Comment on 'Using multiple observationally-based constraints to estimate climate sensitivity' by J. D. Annan and J. C. Hargreaves, Geophys. Res. Lett., doi:10.1029/2005GL025259, 2006. Clim. Past, 6, 411-414.

Hidalgo, H. G., et al., 2009: Detection and attribution of streamflow timing changes to climate change in the Western United States. J. Clim., 22, 3838-3855.

Hodge, S. M., D. C. Trabant, R. M. Krimmel, T. A. Heinrichs, R. S. March, and E. G. Josberger, 1998: Climate variations and changes in mass of three glaciers in western North America. J. Clim., 11, 2161-2179.
Hoekema, D. J., and V. Sridhar, 2011: Relating climatic attributes and water resources allocation: A study using surface water supply and soil moisture indices in the Snake River basin, Idaho. Water Resour. Res., 47, W07536.

Hoerling, M., and A. Kumar, 2003: The perfect ocean for drought. Science, 299, 691-694.

Hoerling, M., et al., 2013: Anatomy of an extreme event. J. Clim., 26, 2811-2832.

Hoerling, M. P., J. K. Eischeid, X.-W. Quan, H. F. Diaz, R. S. Webb, R. M. Dole, and D. R. Easterling, 2012: Is a transition to semipermanent drought conditions imminent in the U.S. great plains? J. Clim., 25, 8380-8386.

Holden, P. B., N. R. Edwards, K. I. C. Oliver, T. M. Lenton, and R. D. Wilkinson, 2010: A probabilistic calibration of climate sensitivity and terrestrial carbon change in GENIE-1. Clim. Dyn., 35, 785-806.

Holland, D. M., R. H. Thomas, B. De Young, M. H. Ribergaard, and B. Lyberth, 2008: Acceleration of Jakobshavn Isbrae triggered by warm subsurface ocean waters. Nature Geosci, 1, 659-664.

Hood, L. L., and R. E. Soukharev, 2012: The lower-stratospheric response to 11-yr solar forcing: Coupling to the troposphere-ocean response. J. Atmos. Sci., 69, 1841-1864.

Hosoda, S., T. Suga, N. Shikama, and K. Mizuno, 2009: Global surface layer salinity change detected by Argo and its implication for hydrological cycle intensification. J. Oceanogr., 65, 579-586.

$\mathrm{Hu}$, Y., and Q. Fu, 2007: Observed poleward expansion of the Hadley circulation since 1979. Atmos. Chem. Phys., 7, 5229-5236.

Hu, Y.Y., C. Zhou, and J. P. Liu, 2011: Observational evidence for poleward expansion of the Hadley circulation. Adv. Atmos. Sci., 28, 33-44.

Hu, Y. Y., L. J. Tao, and J. P. Liu, 2013: Poleward expansion of the Hadley circulation in CMIP5 simulations. Adv. Atmos. Sci., 30, 790-795.

Huber, M., and R. Knutti, 2011: Anthropogenic and natural warming inferred from changes in Earth/'s energy balance. Nature Geosci., 5, 31-36.

Hudson, R. D., M. F. Andrade, M. B. Follette, and A. D. Frolov, 2006: The total ozone field separated into meteorological regimes—Part II: Northern Hemisphere midlatitude total ozone trends. Atmos. Chem. Phys., 6, 5183-5191.

Huntingford, C., P. A. Stott, M. R. Allen, and F. H. Lambert, 2006: Incorporating model uncertainty into attribution of observed temperature change. Geophys. Res. Lett., 33, L05710.

Huss, M., and A. Bauder, 2009: 20th-century climate change inferred from four longterm point observations of seasonal mass balance. Ann. Glaciol., 50, 207-214.

Huss, M., R. Hock, A. Bauder, and M. Funk, 2010: 100-year mass changes in the Swiss Alps linked to the Atlantic Multidecadal Oscillation. Geophys. Res. Lett., 37, L10501.

Huybers, P., 2010: Compensation between model feedbacks and curtailment of climate sensitivity. J. Clim., 23, 3009-3018.

Imbers, J., A. Lopez, C. Huntingford, and M. R. Allen, 2013: Testing the robustness of the anthropogenic climate change detection statements using different empirical models. J. Geophys. Res. Atmos., doi:10.1002/jgrd.50296.

Ineson, S., A. A. Scaife, J. R. Knight, J. C. Manners, N. M. Dunstone, L. J. Gray, and J. D. Haigh, 2011: Solar forcing of winter climate variability in the Northern Hemisphere. Nature Geosci., 4, 753-757.

Ingram, W. J., 2007: Detection and attribution of climate change, and understanding solar influence on climate. In: Solar Variability and Planetary Climates [Y. Calisesi, R.-M. Bonnet , L. Gray , J. Langen, and M. Lockwood (eds.)]. Springer Science+Business Media, New York, NY, USA, and Heidelberg, Germany, pp. 199-211.

IPCC, 2012: Managing the risks of extreme events and disasters to advance climate change adaptation. A Special Report of Working Groups I and II of the Intergovernmental Panel on Climate Change [C. B. Field et al. (eds.)]. Cambridge University Press, Cambridge, UK, and New York, NY, USA, 582.

Ishii, M., and M. Kimoto, 2009: Reevaluation of historical ocean heat content variations with time-varying XBT and $\mathrm{MBT}$ depth bias corrections. J. Oceanogr., 65, 287-299.

Jackson, J. M., E. C. Carmack, F. A. McLaughlin, S. E. Allen, and R. G. Ingram, 2010: Identification, characterization, and change of the near-surface temperature maximum in the Canada Basin, 1993-2008. J. Geophys. Res.J. Geophys. Res. Oceans, 115, C05021.

Jacob, T., J. Wahr, W. T. Pfeffer, and S. Swenson, 2012: Recent contributions of glaciers and ice caps to sea level rise. Nature, 482 514-518.

Jacobs, S. S., A. Jenkins, C. F. Giulivi, and P. Dutrieux, 2011: Stronger ocean circulation and increased melting under Pine Island Glacier ice shelf. Nature Geosci., 4, 519-523. 
Jahn, A., et al., 2012: Late-twentieth-century simulation of Arctic sea-ice and ocean properties in the CCSM4. J. Clim., 25, 1431-1452.

Johannessen, 0. M., et al., 2004: Arctic climate change: Observed and modelled temperature and sea-ice variability Tellus $A, 56,559-560$.

Johanson, C. M., and Q. Fu, 2009: Hadley cell widening: Model simulations versus observations. J. Clim., 22, 2713-2725.

Johnson, G. C., and A. H. Orsi, 1997: Southwest Pacific Ocean water-mass changes between 1968/69 and 1990/91. J. Clim., 10, 306-316.

Jones, G. S., and P. A. Stott, 2011: Sensitivity of the attribution of near surface temperature warming to the choice of observational dataset. Geophys. Res. Lett., 38, L21702.

Jones, G. S., S. F. B. Tett, and P. A. Stott, 2003: Causes of atmospheric temperature change 1960-2000: A combined attribution analysis. Geophys. Res. Lett., 30, 1228.

Jones, G. S., P. A. Stott, and N. Christidis, 2008: Human contribution to rapidly increasing frequency of very warm Northern Hemisphere summers. J. Geophys. Res. Atmos., 113, D02109.

Jones, G. S., N. Christidis, and P. A. Stott, 2011: Detecting the influence of fossil fuel and bio-fuel black carbon aerosols on near surface temperature changes. Atmos. Chem. Phys., 11 799-816.

Jones, G. S., M. Lockwood, and P. A. Stott, 2012: What influence will future solar activity changes over the 21 st century have on projected global near surface temperature changes? J. Geophys. Res. Atmos., 117, D05103.

Jones, G. S., P. A. Stott, and N. Christidis, 2013 Attribution of observed historical near surface temperature variations to anthropogenic and natural causes using CMIP5 simulations. J. Geophys. Res. Atmos., doi:10.1002/jgrd.50239.

Jones, P. D., et al., 2001: Adjusting for sampling density in grid box land and ocean surface temperature time series. J. Geophys. Res. Atmos., 106, 3371-3380.

Joshi, M. M., and G. S. Jones, 2009: The climatic effects of the direct injection of water vapour into the stratosphere by large volcanic eruptions. Atmos. Chem. Phys., 9, 6109-6118.

Joughin, I., and R. B. Alley, 2011: Stability of the West Antarctic ice sheet in a warming world. Nature Geosci., 4, 506-513.

Juckes, M. N., et al., 2007: Millennial temperature reconstruction intercomparison and evaluation. Clim. Past, 3, 591-609.

Jung, M., et al., 2010: Recent decline in the global land evapotranspiration trend due to limited moisture supply. Nature, 467, 951-954.

Jungclaus, J. H., et al., 2010: Climate and carbon-cycle variability over the last millennium. Clim. Past, 6, 723-737.

Kaplan, J. O., K. M. Krumhardt, and N. Zimmermann, 2009: The prehistoric and preindustrial deforestation of Europe. Quat. Sci. Rev., 28, 3016-3034.

Karoly, D. J., and Q. G. Wu, 2005: Detection of regional surface temperature trends. J. Clim., 18, 4337-4343.

Karoly, D. J., and P. A. Stott, 2006: Anthropogenic warming of central England temperature. Atmos. Sci. Lett., 7 81-85.

Karpechko, A. Y., N. P. Gillett, G. J. Marshall, and A. A. Scaife, 2008: Stratospheric influence on circulation changes in the Southern Hemisphere troposphere in coupled climate models. Geophys. Res. Lett., 35, L20806.

Kattsov, V. M., et al., 2010: Arctic sea-ice change: A grand challenge of climate science. J. Glaciol., 56, 1115-1121.

Kaufman, D. S., et al., 2009: Recent warming reverses long-term arctic cooling. Science, 325, 1236-1239.

Kaufmann, R. K., and D. I. Stern, 1997: Evidence for human influence on climate from hemispheric temperature relations. Nature, 388, 39-44.

Kaufmann, R. K., and D. I. Stern, 2002: Cointegration analysis of hemispheric temperature relations. J. Geophys. Res. Atmos., 107, 4012.

Kaufmann, R. K., H. Kauppi, and J. H. Stock, 2006: Emission, concentrations, \& temperature: A time series analysis. Clim. Change, 77, 249-278.

Kaufmann, R. K., H. Kauppi, M. L. Mann, and J. H. Stock, 2011: Reconciling anthropogenic climate change with observed temperature 1998-2008. Proc. Natl. Acad. Sci. U.S.A., 108, 11790-11793.

Kaufmann, R. K., H. Kauppi, M. L. Mann, and J. H. Stock, 2013: Does temperature contain a stochastic trend: Linking statistical results to physical mechanisms. Clim. Change, doi:10.1007/s10584-012-0683-2.

Kay, A. L., S. M. Crooks, P. Pall, and D. A. Stone, 2011 a: Attribution of Autumn/Winter 2000 flood risk in England to anthropogenic climate change: A catchment-based study. J. Hydrol., 406, 97-112.

Kay, J. E., M. M. Holland, and A. Jahn, 2011 b: Inter-annual to multi-decadal Arctic sea ice extent trends in a warming world. Geophys. Res. Lett., 38, L15708.
Keeling, R. F., and H. E. Garcia, 2002: The change in oceanic $\mathrm{O}_{2}$ inventory associated with recent global warming. Proc. Natl. Acad. Sci. U.S.A., 99, 7848-7853.

Keeling, R. F., A. Kortzinger, and N. Gruber, 2010: Ocean deoxygenation in a warming world. Annu. Rev. Mar. Sci., 2, 199-229.

Kettleborough, J. A., B. B. B. Booth, P. A. Stott, and M. R. Allen, 2007: Estimates of uncertainty in predictions of global mean surface temperature. J. Clim., 20, 843-855.

Kharin, V. V., F. W. Zwiers, X. Zhang, and G. C. Hegerl, 2007: Changes in temperature and precipitation extremes in the IPCC ensemble of global coupled model simulations. J. Clim., 20, 1419-1444.

Kharin, V. V., F. W. Zwiers, X. Zhang, and M. Wehner, 2013: Changes in temperature and precipitation extremes in the CMIP5 ensemble. Clim. Change, doi:10.1007/ s10584-013-0705-8.

Kiehl, J. T., 2007: Twentieth century climate model response and climate sensitivity. Geophys. Res. Lett., 34, L22710.

Kinnard, C., C. M. Zdanowicz, D. A. Fisher, E. Isaksson, A. Vernal, and L. G. Thompson, 2011: Reconstructed changes in Arctic sea ice cover over the past 1450 years. Nature, 479, 509-513.

Kirk-Davidoff, D. B., 2009: On the diagnosis of climate sensitivity using observations of fluctuations. Atmos. Chem. Phys., 9, 813-822.

Knight, J., et al., 2009: Do global temperature trends over the last decade falsify climate predictions? In: State of the Climate in 2008. Bull. Am. Meteorol. Soc., 90, S22-S23.

Knight, J. R., C. K. Folland, and A. A. Scaife, 2006: Climate impacts of the Atlantic Multidecadal Oscillation. Geophys. Res. Lett., 33, L17706.

Knight, J. R., R. J. Allan, C. K. Folland, M. Vellinga, and M. E. Mann, 2005: A signature of persistent natural thermohaline circulation cycles in observed climate. Geophys. Res. Lett., 32, L20708.

Knutson, T. R., F. Zeng, and A. T. Wittenberg, 2013: Multi-model assessment of regional surface temperature trends. J. Clim., doi:10.1175/JCLI-D-12-00567.1.

Knutson, T. R., et al., 2010: Tropical cyclones and climate change. Nature Geosci., 3 , 157-163.

Knutti, R., 2008: Why are climate models reproducing the observed global surface warming so well? Geophys. Res. Lett., 35, L18704.

Knutti, R., and G. C. Hegerl, 2008: The equilibrium sensitivity of the Earth's temperature to radiation changes. Nature Geosci., 1, 735-743.

Knutti, R., and L. Tomassini, 2008: Constraints on the transient climate response from observed global temperature and ocean heat uptake. Geophys. Res. Lett., 35, L09701.

Knutti, R., S. Krähenmann, D. J. Frame, and M. R. Allen, 2008: Comment on "Heat capacity, time constant, and sensitivity of Earth's climate system" by S. E. Schwartz. J. Geophys. Res. Atmos., 113, D15103.

Kobashi, T., D. T. Shindell, K. Kodera, J. E. Box, T. Nakaegawa, and K. Kawamura, 2013: On the origin of multidecadal to centennial Greenland temperature anomalies over the past $800 \mathrm{yr}$. Clim. Past, 9, 583-596.

Kobayashi, T., K. Mizuno, and T. Suga, 2012: Long-term variations of surface and intermediate waters in the southern Indian Ocean along $32^{\circ} \mathrm{S}$. J. Oceanogr., 68, 243-265.

Kodama, C., and T. Iwasaki, 2009: Influence of the SST rise on baroclinic instability wave activity under an aquaplanet condition. J. Atmos. Sci, 66, 2272-2287.

Kodera, K., 2004: Solar influence on the Indian Ocean monsoon through dynamical processes. Geophys. Res. Lett., 31, L24209.

Kodera, K., 2006: The role of dynamics in solar forcing. Space Sci. Rev., 23 319-330.

Kodera, K., and Y. Kuroda, 2002: Dynamical response to the solar cycle. J. Geophys. Res. Atmos., 107, 4749.

Kodera, K., K. Coughlin, and O. Arakawa, 2007: Possible modulation of the connection between the Pacific and Indian Ocean variability by the solar cycle. Geophys. Res. Lett., 34, L03710.

Koehler, P., R. Bintanja, H. Fischer, F. Joos, R. Knutti, G. Lohmann, and V. MassonDelmotte, 2010: What caused Earth's temperature variations during the last 800,000 years? Data-based evidence on radiative forcing and constraints on climate sensitivity. Quat. Sci. Rev., 29, 129-145.

Korhonen, H., K. S. Carslaw, P. M. Forster, S. Mikkonen, N. D. Gordon, and H. Kokkola, 2010: Aerosol climate feedback due to decadal increases in Southern Hemisphere wind speeds. Geophys. Res. Lett., 37, L02805.

Krakauer, N. Y., and I. Fung, 2008: Mapping and attribution of change in streamflow in the coterminous United States. Hydrol. Earth Syst. Sci., 12, 1111-1120. 
Kuhlbrodt, T., and J. M. Gregory, 2012: Ocean heat uptake and its consequences for the magnitude of sea level rise and climate change. Geophys. Res. Lett., 39, L18608.

Kunkel, K. E., et al., 2008: Observed changes in weather and climate extremes. In: Weather and Climate Extremes in a Changing Climate. Regions of Focus: North America, Hawaii, Caribbean, and U.S. Pacific Islands [G. A. M. T. R. Karl, C. D. Miller, S. J. Hassol, A. M. Waple, and W. L. Murray (eds.)]. A Report by the U.S. Climate Change Science Program and the Subcommittee on Global Change Research, Washington, DC, pp. 35-80.

Kwok, R., and N. Untersteiner, 2011: The thinning of Arctic sea ice. Physics Today, $64,36-41$.

Kwok, R., G. F. Cunningham, M. Wensnahan, I. Rigor, H. J. Zwally, and D. Yi, 2009: Thinning and volume loss of the Arctic Ocean sea ice cover: 2003-2008. J. Geophys. Res.J. Geophys. Res. Oceans, 114, 007005.

L'Heureux, M., A. H. Butler, B. Jha, A. Kumar, and W. Q. Wang, 2010: Unusual extremes in the negative phase of the Arctic Oscillation during 2009. Geophys. Res. Lett., 37, L10704.

Lamarque, J.-F., et al., 2010: Historical (1850-2000) gridded anthropogenic and biomass burning emissions of reactive gases and aerosols: Methodology and application. Atmos. Chem. Phys., 10, 7017-7039.

Landrum, L., B. L. Otto-Bliesner, E. R. Wahl, A. Conley, P. J. Lawrence, N. Rosenbloom, and H. Teng, 2013: Last millennium climate and its variability in CCSM4. J. Clim., 26, 1085-1111.

Langen, P. L., and V. A. Alexeev, 2007: Polar amplification as a preferred response in an idealized aquaplanet GCM. Clim. Dyn., 29, 305-317.

Latif, M., et al., 2004: Reconstructing, monitoring, and predicting multidecadalscale changes in the North Atlantic thermohaline circulation with sea surface temperature. J. Clim., 17, 1605-1614.

Laxon, S. W., et al., 2013: CryoSat-2 estimates of Arctic sea ice thickness and volume. Geophys. Res. Lett., 40, 732-737.

Lean, J. L., 2006: Comment on "Estimated solar contribution to the global surface warming using the ACRIM TSI satellite composite" by N. Scafetta and B. J. West. Geophys. Res. Lett., 33, L15701.

Lean, J. L., and D. H. Rind, 2008: How natural and anthropogenic influences alter global and regional surface temperatures: 1889 to 2006. Geophys. Res. Lett., 35, L18701.

Lean, J. L., and D. H. Rind, 2009: How will Earth's surface temperature change in future decades? Geophys. Res. Lett., 36, L15708.

Leclercq, P. W., and J. Oerlemans, 2011: Global and hemispheric temperature reconstruction from glacier length fluctuations. Clim. Dyn., 38, 1065-1079.

Ledoit, 0., and M. Wolf, 2004: A well-conditioned estimator for large-dimensional covariance matrices. J. Multivar. Anal., 88, 365-411.

Legras, B., O. Mestre, E. Bard, and P. Yiou, 2010: A critical look at solar-climate relationships from long temperature series. Clim. Past, 6, 745-758.

Leibensperger, E. M., et al., 2012: Climatic effects of 1950-2050 changes in US anthropogenic aerosols-Part 1: Aerosol trends and radiative forcing. Atmos. Chem. Phys., 12, 3333-3348.

Levitus, S., J. I. Antonov, T. P. Boyer, R. A. Locarnini, H. E. Garcia, and A. V. Mishonov, 2009: Global ocean heat content 1955-2008 in light of recently revealed instrumentation problems. Geophys. Res. Lett., 36, L07608.

Lewis, N., 2013: An objective Bayesian, improved approach for applying optimal fingerprint techniques to estimate climate sensitivity. J. Clim., doi:10.1175/JCLID-12-00473.1.

Li, J. P., and J. L. X. L. Wang, 2003: A modified zonal index and its physical sense. Geophys. Res. Lett., 30, 1632.

Libardoni, A. G., and C. E. Forest, 2011: Sensitivity of distributions of climate system properties to the surface temperature dataset. Geophys. Res. Lett., 38, L22705.

Libardoni, A. G., and C. E. Forest, 2013: Correction to "Sensitivity of distributions of climate system properties to the surface temperature dataset". Geophys. Res. Lett., doi:10.1002/grl.50480.

Lin, B., et al., 2010a: Estimations of climate sensitivity based on top-of-atmosphere radiation imbalance. Atmos. Chem. Phys., 10, 1923-1930.

Lin, P., Q. A. Fu, S. Solomon, and J. M. Wallace, 2010b: Temperature trend patterns in Southern Hemisphere high latitudes: Novel indicators of stratospheric change $J$. Clim., 22, 6325-6341.

Lindsay, R. W., J. Zhang, A. Schweiger, M. Steele, and H. Stern, 2009: Arctic sea ice retreat in 2007 follows thinning trend. J. Clim., 22, 165-176.

Lindzen, R. S., and Y. S. Choi, 2009: On the determination of climate feedbacks from ERBE data. Geophys. Res. Lett., 36, L16705.
Lindzen, R. S., and Y. S. Choi, 2011: On the observational determination of climate sensitivity and its implications. Asia-Pacific J. Atmos. Sci., 47, 377-390.

Liu, C., R. P. Allan, and G. J. Huffman, 2012: Co-variation of temperature and precipitation in CMIP5 models and satellite observations. Geophys. Res. Lett., 39, L13803.

Lockwood, M., 2008: Recent changes in solar outputs and the global mean surface temperature. III. Analysis of contributions to global mean air surface temperature rise. Proc. R. Soc. London A, 464, 1387-1404.

Lockwood, M., 2012: Solar influence on global and regional climates. Surv. Geophys., 33, 503-534.

Lockwood, M., and C. Fröhlich, 2007: Recent oppositely directed trends in solar climate forcings and the global mean surface air temperature Proc. $R$. Soc. London $A, 463,2447-2460$.

Lockwood, M., and C. Fröhlich, 2008: Recent oppositely directed trends in solar climate forcings and the global mean surface air temperature: II. Different reconstructions of the total solar irradiance variation and dependence on response time scale. Proc. R. Soc. London A, 464, 1367-1385.

Lockwood, M., R. G. Harrison, T. Woollings, and S. K. Solanki, 2010: Are cold winters in Europe associated with low solar activity? Environ. Res. Lett., 5, 024001.

Loehle, C., and N. Scaffetta, 2011: Climate change attribution using empirical decomposition of climatic data. Open Atmos. Sci. J., 5, 74-86.

Lott, F. C., et al., 2013: Models versus radiosondes in the free atmosphere: A new detection and attribution analysis of temperature. J. Geophys. Res. Atmos., 118, 2609-2619.

Lu, J., G. A. Vecchi, and T. Reichler, 2007: Expansion of the Hadley cell under global warming. Geophys. Res. Lett., 34, L06805.

Lu, J., G. Chen, and D. M. W. Frierson, 2008: Response of the zonal mean atmospheric circulation to El Nino versus global warming. J. Clim., 21, 5835-5851.

Lu, J., C. Deser, and T. Reichler, 2009: Cause of the widening of the tropical belt since 1958. Geophys. Res. Lett., 36, L03803.

Lucas, C., H. Nguyen, and B. Timbal, 2012: An observational analysis of Southern Hemisphere tropical expansion. J. Geophys. Res. Atmos., 117, D17112.

Lunt, D. J., A. M. Haywood, G. A. Schmidt, U. Salzmann, P. J. Valdes, and H. J. Dowsett, 2010: Earth system sensitivity inferred from Pliocene modelling and data. Nature Geosci., 3, 60-64.

Luterbacher, J., D. Dietrich, E. Xoplaki, M. Grosjean, and H. Wanner, 2004: European seasonal and annual temperature variability, trend, and extremes since 1500. Science, 303, 1499-1503.

Mahajan, S., R. Zhang, and T. L. Delworth, 2011: Impact of the Atlantic Meridional Overturning Circulation (AMOC) on Arctic surface air temperature and sea ice variability. J. Clim., 24, 6573-6581.

Mahlstein, I., and R. Knutti, 2012 September Arctic sea ice predicted to disappear for 20C global warming above present. J. Geophys. Res. Atmos., 117, D06104.

Mahlstein, I., G. Hegerl, and S. Solomon, 2012: Emerging local warming signals in observational data. Geophys. Res. Lett., 39, L21711.

Mahlstein, I., R. Knutti, S. Solomon, and R. W. Portmann, 2011: Early onset of significant local warming in low latitude countries. Environ. Res. Lett., 6, 034009.

Manabe, S., and R. T. Wetherald, 1975: The effects of doubling the $\mathrm{CO}_{2}$ concentration on the climate of a General Circulation Model. J. Atmos. Sci., 32, 3-15.

Mankoff, K. D., S. S. Jacobs, S. M. Tulaczyk, and S. E. Stammerjohn, 2012: The role of Pine Island Glacier ice shelf basal channels in deep water upwelling, polynyas and ocean circulation in Pine Island Bay, Antarctica. Ann. Glaciol., 53, 123-128.

Mann, M. E., 2011: On long range temperature dependence in global surface temperature series. Clim. Change, 107, 267-276.

Mann, M. E., and K. A. Emanuel, 2006: Atlantic hurricane trends linked to climate change. Eos Trans. Am. Geophys. Union, 87, 233-238.

Mann, M. E., Z. H. Zhang, M. K. Hughes, R. S. Bradley, S. K. Miller, S. Rutherford, and F. B. Ni, 2008: Proxy-based reconstructions of hemispheric and global surface temperature variations over the past two millennia. Proc. Natl. Acad. Sci. U.S.A., 105, 13252-13257.

Mann, M. E., et al., 2009: Global signatures and dynamical origins of the Little Ice age and medieval climate anomaly. Science, 326, 1256-1260.

Marcott, S. A., J. D. Shakun, P. U. Clark, and A. C. Mix, 2013: A reconstruction of regional and global temperature for the past 11,300 years. Science, 339, 11981201

Marzeion, B., and A. Nesje, 2012: Spatial patterns of North Atlantic Oscillation influence on mass balance variability of European glaciers. Cryosphere, 6, 661-673. 
Maslanik, J. A., C. Fowler, J. Stroeve, S. Drobot, J. Zwally, D. Yi, and W. Emery, 2007: A younger, thinner Arctic ice cover: Increased potential for rapid, extensive sea-ice loss. Geophys. Res. Lett., 34, L24501.

Maslowski, W., J. C. Kinney, M. Higgins, and A. Roberts, 2012: The future of Arctic sea ice. Annu. Rev. Earth Planet. Sci., 40, 625-654.

Massey, N., T. Anna, R. C., F. E. L. Otto, S. Wilson, R. G. Jones, and M. R. Allen, 2012: Have the odds of warm November temperatures and of cold December temperatures in central england changed? Bull. Am. Meteorol. Soc., 93, 10571059.

Mastrandrea, M. D., et al., 2011: Guidance note for lead authors of the IPCC Fifth Assessment Report on consistent treatment of uncertainties. Intergovernmental Panel on Climate Change (IPCC), Geneva, Switzerland.

Matthes, K., Y. Kuroda, K. Kodera, and U. Langematz, 2006: Transfer of the solar signal from the stratosphere to the troposphere: Northern winter. J. Geophys. Res. Atmos., 111 D06108.

Matthews, H. D., N. P. Gillett, P. A. Stott, and K. Zickfeld, 2009: The proportionality of global warming to cumulative carbon emissions. Nature, 459, 829-U3.

Mazzarella, A., and N. Scafetta, 2012: Evidences for a quasi 60-year North Atlantic Oscillation since 1700 and its meaning for global climate change. Theor. Appl. Climatol., 107, 599-609.

McCracken, K. G., and J. Beer, 2007: Long-term changes in the cosmic ray intensity at Earth, 1428-2005. J. Geophys. Res. Space Physics, 112, A10101.

McKitrick, R., and L. Tole, 2012: Evaluating explanatory models of the spatial pattern of surface climate trends using model selection and Bayesian averaging methods. Clim. Dyn., 39, 2867-2882.

McKitrick, R., S. McIntyre, and C. Herman, 2010: Panel and multivariate methods for tests of trend equivalence in climate data series. Atmos. Sci. Lett., 11, 270-277.

McLandress, C., J. Perlwitz, and T. G. Shepherd, 2012: Comment on "Tropospheric temperature response to stratospheric ozone recovery in the 21 st century" by Hu et al. , 2011. Atmos. Chem. Phys., 12, 2533-2540.

McLandress, C., T. G. Shepherd, J. F. Scinocca, D. A. Plummer, M. Sigmond, A. I. Jonsson, and M. C. Reader, 2011: Separating the dynamical effects of climate change and ozone depletion. Part II: Southern Hemisphere troposphere. J. Clim., 24, 1850-1868.

Mecking, S., M. J. Warner, and J. L. Bullister, 2006: Temporal changes in pCFC-12 ages and AOU along two hydrographic sections in the eastern subtropical North Pacific. Deep-Sea Res. Pt. I, 53, 169-187.

Meehl, G. A., and J. M. Arblaster, 2009: A lagged warm event-like response to peaks in solar forcing in the Pacific region. J. Clim., 22, 3647-3660.

Meehl, G. A., J. M. Arblaster, and C. Tebaldi, 2007a: Contributions of natural and anthropogenic forcing to changes in temperature extremes over the U.S. Geophys. Res. Lett., 34, L19709.

Meehl, G. A., J. M. Arblaster, G. Branstator, and H. van Loon, 2008: A coupled air-sea response mechanism to solar forcing in the Pacific region. J. Clim., $212883-$ 2897.

Meehl, G. A., W. M. Washington, T. M. L. Wigley, J. M. Arblaster, and A. Dai, 2003: Solar and greenhouse gas forcing and climate response in the 20th century. J. Clim., 16 426-444.

Meehl, G. A., J. M. Arblaster, K. Matthes, F. Sassi, and H. van Loon, 2009: Amplifying the Pacific climate system response to a small 11-747year solar cycle forcing. Science, 325 1114-1118.

Meehl, G. A., et al., 2007b: Global climate projections. In: Climate Change 2007: The Physical Science Basis. Contribution of Working Group I to the Fourth Assessment Report of the Intergovernmental Panel on Climate Change [Solomon, S., D. Qin, M. Manning, Z. Chen, M. Marquis, K. B. Averyt, M. Tignor and H. L. Miller (eds.)] Cambridge University Press, Cambridge, United Kingdom and New York, NY, USA, pp. 747-846.

Meinshausen, M., et al., 2009: Greenhouse-gas emission targets for limiting global warming to $2^{\circ} \mathrm{C}$. Nature, $458,1158-1162$.

Merrifield, M., and M. Maltrud, 2011: Regional sea level trends due to a Pacific trade wind intensification. Geophys. Res. Lett., 38, L21605.

Meyssignac, B., D. Salas y Melia, M. Becker, W. Llovel, and A. Cazenave, 2012: Tropical Pacific spatial trend patterns in observed sea level: Internal variability and/or anthropogenic signature? Clim. Past, 8, 787-802.

Miller, G. H., R. B. Alley, J. Brigham-Grette, J. J. Fitzpatrick, L. Polyak, M. C. Serreze, and J.W. C. White, 2010: Arctic amplification: Can the past constrain the future? Quat. Sci. Rev., 29, 1779-1790.

Miller, G. H., et al., 2012: Abrupt onset of the Little Ice Age triggered by volcanism and sustained by sea-ice/ocean feedbacks. Geophys. Res. Lett., 39, L02708.
Miller, R. L., G. A. Schmidt, and D. T. Shindell, 2006: Forced annular variations in the 20th century intergovernmental panel on climate change fourth assessment report models. J. Geophys. Res. Atmos., 111, D18101.

Mills, T. C., 2009: How robust is the long-run relationship between temperature and radiative forcing? Clim. Change, 94, 351-361.

Min, S.-K., and A. Hense, 2006: A Bayesian assessment of climate change using multimodel ensembles. Part I: Global mean surface temperature. J. Clim., 19, 3237-3256.

Min, S.-K., and A. Hense, 2007: A Bayesian assessment of climate change using multimodel ensembles. Part II: Regional and seasonal mean surface temperatures. J. Clim., 20, 2769-2790.

Min, S.-K., and S.-W. Son, 2013: Multi-model attribution of the Southern Hemisphere Hadley cell widening: Major role of ozone depletion. J. Geophys. Res. Atmos., 118, 3007-3015.

Min, S.-K., X. B. Zhang, and F. Zwiers, 2008a: Human-induced arctic moistening. Science, 320, 518-520.

Min, S.-K., X. B. Zhang, F. W. Zwiers, and T. Agnew, 2008b: Human influence on Arctic sea ice detectable from early 1990s onwards. Geophys. Res. Lett., 35, L21701.

Min, S.-K., X. Zhang, F. W. Zwiers, and G. C. Hegerl, 2011: Human contribution to more intense precipitation extremes. Nature, 470, 378-381.

Min, S.-K., X. Zhang, F. W. Zwiers, P. Friederichs, and A. Hense, 2008c: Signal detectability in extreme precipitation changes assessed from twentieth century climate simulations. Clim. Dyn., 32, 95-111.

Min, S.-K., X. Zhang, F. Zwiers, H. Shiogama, Y.-S. Tung, and M. Wehner, 2013: Multimodel detection and attribution of extreme temperature changes. J. Clim., doi:10.1175/JCLI-D-12-00551.w.

Misios, S., and H. Schmidt, 2012: Mechanisms involved in the amplification of the 11-yr solar cycle signal in the Tropical Pacific ocean. J. Clim., 25, 5102-5118.

Mitchell, D. M., P. A. Stott, L. J. Gray, F. C. Lott, N. Butchart, S. C. Hardiman, and S. M. Osprey, 2013: The impact of stratospheric resolution on the detectability of climate change signals in the free atmosphere. Geophys. Res. Lett., 40, 937-942.

Miyazaki, C., and T. Yasunari, 2008: Dominant interannual and decadal variability of winter surface air temperature over Asia and the surrounding oceans. J. Clim., 21, 1371-1386.

Moberg, A., D. M. Sonechkin, K. Holmgren, N. M. Datsenko, and W. Karlen, 2005: Highly variable Northern Hemisphere temperatures reconstructed from low- and high-resolution proxy data. Nature, 433, 613-617.

Mölg, T., N. J. Cullen, D. R. Hardy, M. Winkler, and G. Kaser, 2009: Quantifying climate change in the tropical midtroposphere over East Africa from glacier shrinkage on Kilimanjaro. J. Clim., 22, 4162-4181.

Mölg, T., M. Großhauser, A. Hemp, M. Hofer, and B. Marzeion, 2012: Limited forcing of glacier loss through land-cover change on Kilimanjaro. Nature Clim. Change, 2, 254-258.

Morak, S., G. C. Hegerl, and J. Kenyon, 2011: Detectable regional changes in the number of warm nights. Geophys. Res. Lett., 38, L17703.

Morak, S., G. C. Hegerl, and N. Christidis, 2013: Detectable changes in the frequency of temperature extremes. J. Clim., 26, 1561-1574.

Morgenstern, O., et al., 2010: Anthropogenic forcing of the Northern Annular Mode in CCMVal-2 models. J. Geophys. Res. Atmos., 115, D00M03.

Morice, C. P., J. J. Kennedy, N. A. Rayner, and P. D. Jones, 2012: Quantifying uncertainties in global and regional temperature change using an ensemble of observational estimates: The HadCRUT4 data set. J. Geophys. Res. Atmos., 117, D08101.

Murphy, D. M., and P. M. Forster, 2010: On the accuracy of deriving climate feedback parameters from correlations between surface temperature and outgoing radiation. J. Clim., 23 4983-4988.

Murphy, D. M., S. Solomon, R. W. Portmann, K. H. Rosenlof, P. M. Forster, and T. Wong, 2009: An observationally based energy balance for the Earth since 1950. J. Geophys. Res. Atmos., 114, D17107.

Nagato, Y., and H. L. Tanaka, 2012: Global warming trend without the contribution from decadal variability of the Arctic oscillation. Polar Sci, 6, 15-22.

Nakanowatari, T., K. I. Ohshima, and M. Wakatsuchi, 2007: Warming and oxygen decrease of intermediate water in the northwestern North Pacific, originating from the Sea of Okhotsk, 1955-2004. Geophys. Res. Lett., 34, L04602.

National Research Council, 2012: The Effects of Solar Variability on Earth's Climate: A Workshop Report. The National Academies Press, Washington, DC, 70 pp.

Nesje, A., O. Lie, and S. O. Dahl, 2000: Is the North Atlantic Oscillation reflected in Scandinavian glacier mass balance records? J. Quat. Sci., 15, 587-601. 
Nghiem, S. V., I. G. Rigor, D. K. Perovich, P. Clemente-Colon, J. W. Weatherly, and G. Neumann, 2007: Rapid reduction of Arctic perennial sea ice. Geophys. Res. Lett., 34, L19504.

Nguyen, H., B. Timbal, A. Evans, C. Lucas, and I. Smith, 2013: The Hadley circulation in reanalyses: Climatology, variability and change. J. Clim., 26, 3357-3376.

Noake, K., D. Polson, G. Hegerl, and X. Zhang, 2012: Changes in seasonal land precipitation during the latter 20th Century. Geophys. Res. Lett., 39, L03706.

North, G. R., and M. J. Stevens, 1998: Detecting climate signals in the surface temperature record. J. Clim., 11, 563-577.

Notz, D., and J. Marotzke, 2012: Observations reveal external driver for Arctic sea-ice retreat. Geophys. Res. Lett., 39, L08502.

Nussbaumer, S. U., and H. J. Zumbühl, 2012: The Little Ice Age history of the Glacier des Bossons (Mont Blanc massif, France): A new high-resolution glacier length curve based on historical documents. Clim. Change, 111, 301-334.

0'Gorman, P. A., and T. Schneider, 2008: Energy of midlatitude transient eddies in idealized simulations of changed climates. J. Clim., 21, 5797-5806.

O'Gorman, P. A., 2010: Understanding the varied response of the extratropical storm tracks to climate change. Proc. Natl. Acad. Sci. U.S.A., 107, 19176-19180.

Oerlemans, J., 2005: Extracting a climate signal from 169 glacier records. Science, 308, 675-677.

Olson, R., R. Sriver, M. Goes, N. M. Urban, H. D. Matthews, M. Haran, and K. Keller, 2012: A climate sensitivity estimate using Bayesian fusion of instrumental observations and an Earth System model. J. Geophys. Res. Atmos., 117, D04103.

Ono, T., T. Midorikawa, Y. W. Watanabe, K. Tadokoro, and T. Saino, 2001: Temporal increases of phosphate and apparent oxygen utilization in the subsurface waters of western subarctic Pacific from 1968 to 1998. Geophys. Res. Lett., 28, 3285-3288.

Otto-Bliesner, B. L., et al., 2009: A comparison of PMIP2 model simulations and the MARGO proxy reconstruction for tropical sea surface temperatures at last glacial maximum. Clim. Dyn., 32, 799-815.

Otto, A., et al., 2013: Energy budget constraints on climate response. Nature Geosci., $6,415-416$.

Otto, F. E. L., N. Massey, G. J. van Oldenborgh, R. G. Jones, and M. R. Allen, 2012: Reconciling two approaches to attribution of the 2010 Russian heat wave. Geophys. Res. Lett., 39, L04702.

Overland, J. E., 2009: The case for global warming in the Arctic. In: Influence of Climate Change on the Changing Arctic and Sub-Arctic Conditions. NATO Science for Peace and Security Series C: Environmental Security [J. C. J. Nihoul and A. G. Kostianoy (eds.)]. Springer Science+Business Media, Dordrecht, Netherlands, pp. 13-23.

Overland, J. E., and M. Wang, 2013: When will the summer arctic be nearly sea ice free? Geophys. Res. Lett., doi:10.1002/grl.50316.

Overland, J. E., M. Wang, and S. Salo, 2008: The recent Arctic warm period. Tellus A, 60, 589-597.

Overland, J. E., K. R. Wood, and M. Wang, 2011:Warm Arctic-cold continents: Climate impacts of the newley open Arctic sea. Polar Res., 30, 15787.

Overland, J. E., J. A. Francis, E. Hanna, and W. M., 2012: The recent shift in early summer Arctic atmospheric circulation. Geophys. Res. Lett., 39, L19804.

Oza, S. R., R. K. K. Singh, N. K. Vyas, and A. Sarkar, 2011a: Spatio-Temporal analysis of melting onset dates of sea-ice in the Arctic. Indian J. Geo-Mar. Sci., 40, 497-501.

Oza, S. R., R. K. K. Singh, A. Srivastava, M. K. Dash, I. M. L. Das, and N. K. Vyas, 2011b: Inter-annual variations observed in spring and summer antarctic sea ice extent in recent decade. Mausam, 62, 633-640.

Padilla, L. E., G. K. Vallis, and C. W. Rowley, 2011: Probabilistic estimates of transient climate sensitivity subject to uncertainty in forcing and natural variability. J. Clim., 24, 5521-5537.

Pagani, M., K. Caldeira, R. Berner, and D. J. Beerling, 2009: The role of terrestrial plants in limiting atmospheric $\mathrm{CO}_{2}$ decline over the past 24 million years. Nature, $460,85-88$.

PAGES 2k Consortium, 2013: Continental-scale temperature variability during the past two millennia. Nature Geosci., 6, 339-346.

Palastanga, V., G. van der Schrier, S. L. Weber, T. Kleinen, K. R. Briffa, and T. J. Osborn, 2011: Atmosphere and ocean dynamics: Contributors to the Little Ice Age and Medieval Climate Anomaly. Clim. Dyn., 36, 973-987.

Paleosens Members, 2012: Making sense of palaeoclimate sensitivity. Nature, 491, 683-691.

Pall, P., et al., 2011: Anthropogenic greenhouse gas contribution to UK autumn flood risk. Nature, 470, 382-385.
Palmer, M. D., S. A. Good, K. Haines, N. A. Rayner, and P. A. Stott, 2009: A new perspective on warming of the global oceans. Geophys. Res. Lett., 36, L20709.

Penner, J. E., M. Wang, A. Kumar, L. Rotstayn, and B. Santer, 2007: Effect of black carbon on mid-troposphere and surface temperature trends. In: Human-Induced Climate Change: An Interdisciplinary Assessment [M. Schlesinger, et al. (ed.)], Cambridge University Press, Cambridge, United Kingdom, and New York, NY, USA, pp. 18-33.

Peterson, T. C., P. A. Stott, and S. Herring, 2012: Explaining extreme events of 2011 from a climate perspective. Bull. Am. Meteorol. Soc., 93, 1041-1067.

Pierce, D. W., T. P. Barnett, B. D. Santer, and P. J. Gleckler, 2009: Selecting global climate models for regional climate change studies. Proc. Natl. Acad. Sci. U.S.A., $106,8441-8446$

Pierce, D. W., P. J. Gleckler, T. P. Barnett, B. D. Santer, and P. J. Durack, 2012: The fingerprint of human-induced changes in the ocean's salinity and temperature fields. Geophys. Res. Lett., 39, L21704.

Pierce, D. W., T. P. Barnett, K. AchutaRao, P. Gleckler, J. Gregory, and W. Washington, 2006: Anthropogenic warming of the oceans: Observations and model results. J. Clim., 19, 1873-1900.

Pierce, D. W., et al., 2008: Attribution of declining Western U.S. snowpack to human effects. J. Clim., 21, 6425-6444.

Pitman, A. J., et al., 2009: Uncertainties in climate responses to past land cover change: First results from the LUCID intercomparison study. Geophys. Res. Lett., 36, L14814.

Po-Chedley, S., and Q. Fu, 2012: Discrepancies in tropical upper tropospheric warming between atmospheric circulation models and satellites. Environ. Res. Lett., 7, 044018.

Polson, D., G. C. Hegerl, X. Zhang, and T. J. Osborn, 2013: Causes of robust seasonal land precipitation changes. J. Clim., doi:10.1175/JCLI-D-12-00474.1.

Polvani, L. M., D. W. Waugh, G. J. P. Correa, and S. W. Son, 2011: Stratospheric ozone depletion: The main driver of twentieth-century atmospheric circulation changes in the southern hemisphere. J. Clim., 24, 795-812.

Polyakov, I. V., J. E. Walsh, and R. Kwok, 2012: Recent changes of Arctic multiyear sea ice coverage and the likely causes. Bull. Am. Meteorol. Soc., 93, 145-151.

Polyakov, I. V., U. S. Bhatt, H. L. Simmons, D. Walsh, J. E. Walsh, and X. Zhang, 2005: Multidecadal variability of North Atlantic temperature and salinity during the twentieth century. J. Clim., 18, 4562-4581.

Polyakov, I. V., et al., 2003: Variability and trends of air temperature and pressure in the maritime Arctic, 1875-2000. J. Clim., 16, 2067-2077.

Pongratz, J., C. H. Reick, T. Raddatz, and M. Claussen, 2009: Effects of anthropogenic land cover change on the carbon cycle of the last millennium. Global Biogeochem. Cycles, 23, GB4001.

Pritchard, H. D., S. R. M. Ligtenberg, H. A. Fricker, D. G. Vaughan, M. R. van den Broeke, and L. Padman, 2012: Antarctic ice sheet loss driven by basal melting of ice shelves. Nature, 484, 502-505.

Pueyo, S., 2012: Solution to the paradox of climate sensitivity. Clim. Change, 113, 163-179

Quadrelli, R., and J. M. Wallace, 2004: A simplified linear framework for interpreting patterns of Northern Hemisphere wintertime climate variability. J. Clim., 17, 3728-3744.

Rahmstorf, S., and D. Coumou, 2011: Increase of extreme events in a warming world. Proc. Natl. Acad. Sci. U.S.A., 108, 17905-17909.

Ramaswamy, V., M. D. Schwarzkopf, W. J. Randel, B. D. Santer, B. J. Soden, and G. L. Stenchikov, 2006: Anthropogenic and natural influences in the evolution of lower stratospheric cooling. Science, 311, 1138-1141.

Ramsay, H. A., and A. H. Sobel, 2011: The effects of relative and absolute sea surface temperature on tropical cyclone potential intensity using a single column model. J. Clim., 24, 183-193.

Randel, W. J., et al., 2009: An update of observed stratospheric temperature trends. J. Geophys. Res. Atmos., 114, D02107.

Ray, E. A., et al., 2010: Evidence for changes in stratospheric transport and mixing over the past three decades based on multiple data sets and tropical leaky pipe analysis. J. Geophys. Res. Atmos., 115, D21304.

Rea, W., M. Reale, and J. Brown, 2011: Long memory in temperature reconstructions. Clim. Change, 107, 247-265.

Reichert, B. K., L. Bengtsson, and J. Oerlemans, 2002: Recent glacier retreat exceeds internal variability. J. Clim., 15, 3069-3081.

Ribes, A., and L. Terray, 2013: Application of regularised optimal fingerprint analysis for attribution. Part II: Application to global near-surface temperature Clim. Dyn., doi:10.1007/s00382-013-1736-6. 
Ribes, A., J. M. Azais, and S. Planton, 2009: Adaptation of the optimal fingerprint method for climate change detection using a well-conditioned covariance matrix estimate. Clim. Dyn., 33, 707-722.

Ribes, A., J. M. Azais, and S. Planton, 2010: A method for regional climate change detection using smooth temporal patterns. Clim. Dyn., 35, 391-406.

Ribes, A., S. Planton, and L. Terray, 2013: Application of regularised optimal fingerprint for attribution. Part I: Method, properties and idealised analysis. Clim. Dyn., doi:10.1007/s00382-013-1735-7.

Rind, D., J. Lean, J. Lerner, P. Lonergan, and A. Leboisitier, 2008: Exploring the stratospheric/troposheric response to solar forcing. J. Geophys. Res. Atmos., 113 D24103.

Ring, M. J., D. Lindner, E. F. Cross, and M. E. Schlesinger, 2012: Causes of the global warming observed since the 19th century. atmospheric and climate sciences Atmos. Clim. Sci., 2, 401-415.

Roe, G. H., and M. B. Baker, 2007: Why is climate sensitivity so unpredictable? Science, 318, 629-632.

Roe, G. H., and M. A. O'Neal, 2009: The response of glaciers to intrinsic climate variability: Observations and models of late-Holocene variations in the Pacific Northwest. J. Glaciol., 55, 839-854.

Roemmich, D., and J. Gilson, 2009: The 2004-2008 mean and annual cycle of temperature, salinity, and steric height in the global ocean from the Argo Program. Prog. Oceanogr., 82, 81-100.

Rogelj, J., M. Meinshausen, and R. Knutti, 2012: Global warming under old and new scenarios using IPCC climate sensitivity range estimates. Nature Clim. Change, 2, 248-253.

Rohde, R., et al., 2013: A new estimate of the average Earth surface land temperature spanning 1753 to 2011. Geoinf. Geostat. Overview, 1, 1.

Roscoe, H. K., and J. D. Haigh, 2007: Influences of ozone depletion, the solar cycle and the QBO on the Southern Annular Mode. Q. J. R. Meteorol. Soc., 133, 18551864.

Roy, I., and J. D. Haigh, 2010: Solar cycle signals in sea level pressure and sea surface temperature. Atmos. Chem. Phys., 10 3147-3153.

Roy, I., and J. D. Haigh, 2012: Solar cycle signals in the Pacific and the issue of timings. J. Atmos. Sci., 69 1446-1451.

Royer, D. L., 2008: Linkages between $\mathrm{CO}_{2}$, climate, and evolution in deep time. Proc. Natl. Acad. Sci. U.S.A., 105, 407-408.

Royer, D. L., R. A. Berner, and J. Park, 2007: Climate sensitivity constrained by $\mathrm{CO}_{2}$ concentrations over the past 420 million years. Nature, 446, 530-532.

Rupp, D. E., P. W. Mote, N. L. Bindoff, P. A. Stott, and D. A. Robinson, 2013: Detection and attribution of observed changes in Northern Hemisphere spring snow cover. J. Clim., doi:10.1175/JCLI-D-12-00563.1.

Rupp, D. E., P.W. Mote, N. Massey, J. R. Cameron, R. Jones, and M. R. Allen, 2012: Did human influence on climate make the 2011 Texas drought more probable? Bull. Am. Meteorol. Soc., 93, 1052-1054.

Sanso, B., and C. Forest, 2009: Statistical calibration of climate system properties. J. R. Stat. Soc. C, 58, 485-503.

Santer, B. D., W. Bruggemann, U. Cubasch, K. Hasselmann, H. Hock, E. Maierreimer, and U. Mikolajewicz, 1994: Signal-to-noise analysis of time-dependent greenhouse warming experiments: 1. Pattern-analysis. Clim. Dyn., 9, 267-285.

Santer, B. D., et al., 2009: Incorporating model quality information in climate change detection and attribution studies. Proc. Natl. Acad. Sci. U.S.A., 106 14778-14783.

Santer, B. D., et al., 2007: Identification of human-induced changes in atmospheric moisture content. Proc. Natl. Acad. Sci. U.S.A., 104, 15248-15253.

Santer, B. D., et al., 2006: Forced and unforced ocean temperature changes in Atlantic and Pacific tropical cyclogenesis regions. Proc. Natl. Acad. Sci. U.S.A., 103, 13905-13910.

Santer, B. D., et al., 2013: Identifying human influences on atmospheric temperature. Proc. Natl. Acad. Sci. U.S.A., 110, 26-33.

Sato, M., J. E. Hansen, M. P. McCormick, and J. B. Pollack, 1993: Stratospheric aerosol optical depth, 1850-1990. J. Geophys. Res. Atmos., 98, 22987-22994.

Scafetta, N., and B. J. West, 2007: Phenomenological reconstructions of the solar signature in the Northern Hemisphere surface temperature records since 1600. J. Geophys. Res. Atmos., 112, D24S03.

Scheff, J., and D. M. W. Frierson, 2012a: Robust future precipitation declines in CMIP5 largely reflect the poleward explansion of the model subtropical dry zones. Geophys. Res. Lett., 39, L18704.

Scheff, J., and D. M. W. Frierson, 2012b: Twenty-first-century multimodel subtropical precipitation declines are mostly midlatitude shifts. J. Clim., 25, 4330-434.
Schlesinger, M. E., and N. Ramankutty, 1994: An oscillation in the global climate system of period $65-70$ years. Nature, 367, 723-726.

Schmidt, G., et al., 2012: Climate forcing reconstructions for use in PMIP simulations of the last millennium (v1.1). Geosci. Model Dev., 5, 185-191.

Schmidt, G. A., et al., 2011: Climate forcing reconstructions for use in PMIP simulations of the last millennium (v1.0). Geosci. Model Dev., 4, 33-45.

Schmittner, A., et al., 2011: Climate sensitivity estimated from temperature reconstructions of the last glacial maximum. Science, 334, 1385-1388.

Schmittner, A., et al., 2012: Response to comment on "Climate sensitivity estimated from temperature reconstructions of the Last Glacial Maximum". Science, 337 1294

Schneider, T., and I. M. Held, 2001: Discriminants of twentieth-century changes in earth surface temperatures. J. Clim., 14 249-254.

Schneider von Deimling, T., H. Held, A. Ganopolski, and S. Rahmstorf, 2006: Climate sensitivity estimated from ensemble simulations of glacial climate. Clim. Dyn., 27, 149-163.

Schnur, R., and K. I. Hasselmann, 2005: Optimal filtering for Bayesian detection and attribution of climate change. Clim. Dyn., 24, 45-55.

Schubert, S., et al., 2009: A US CLIVAR project to assess and compare the responses of global climate models to drought-related sst forcing patterns: Overview and results. J. Clim., 22, 5251-5272.

Schurer, A., G. Hegerl, M. E. Mann, S. F. B. Tett, and S. J. Phipps, 2013: Separating forced from chaotic climate variability over the past millennium. J. Clim., doi:10.1175/JCLI-D-12-00826.1.

Schwartz, S. E., 2007: Heat capacity, time constant, and sensitivity of Earth's climate system. J. Geophys. Res. Atmos., 112, D24S05.

Schwartz, S. E., 2012: Determination of Earth's transient and equilibrium climate sensitivities from observations over the twentieth century: Strong dependence on assumed forcing. Surv. Geophys., 33 745-777.

Schwartz, S. E., R. J. Charlson, and H. Rodhe, 2007: Quantifying climate change-too rosy a picture? Nature Rep. Clim. Change, doi:10.1038/climate.2007.22, 23-24.

Schwartz, S. E., R. J. Charlson, R. A. Kahn, J. A. Ogren, and H. Rodhe, 2010: Why hasn't Earth warmed as much as expected? J. Clim., 23, 2453-2464.

Schweiger, A., R. Lindsay, J. Zhang, M. Steele, H. Stern, and R. Kwok, 2011: Uncertainty in modeled Arctic sea ice volume. J. Geophys. Res.J. Geophys. Res. Oceans, 116, C00D06.

Screen, J. A., and I. Simmonds, 2010: Increasing fall-winter energy loss from the Arctic Ocean and its role in Arctic temperature amplification. Geophys. Res. Lett., 37, L16707.

Seager, R., N. Naik, and G. A. Vecchi, 2010: Thermodynamic and dynamic mechanisms for large-scale changes in the hydrological cycle in response to global warming. J. Clim., 23, 4651-4668.

Seager, R., Y. Kushnir, C. Herweijer, N. Naik, and J. Velez, 2005: Modeling of tropical forcing of persistent droughts and pluvials over western North America: 18562000. J. Clim., 18, 4065-4088.

Sedlacek, K., and R. Knutti, 2012: Evidence for external forcing on 20th-century climate from combined ocean atmosphere warming patterns. Geophys. Res. Lett., 39, L20708.

Seidel, D. J., and W. J. Randel, 2007: Recent widening of the tropical belt: Evidence from tropopause observations. J. Geophys. Res. Atmos., 112, D20113.

Seidel, D. J., Q. Fu, W. J. Randel, and T. J. Reichler, 2008: Widening of the tropical belt in a changing climate. Nature Geosci., 1, 21-24.

Seidel, D. J., N. P. Gillett, J. R. Lanzante, K. P. Shine, and P. W. Thorne, 2011: Stratospheric temperature trends: Our evolving understanding. WIREs Clim. Change, 2, 592-616.

Seidel, D. J., Y. Zhang, A. Beljaars, J.-C. Golaz, A. R. Jacobson, and B. Medeiros, 2012: Climatology of the planetary boundary layer over the continental United States and Europe. J. Geophys. Res. Atmos., 117, D17106.

Semenov, V. A., 2008: Influence of oceanic inflow to the Barents Sea on climate variability in the Arctic region. Doklady Earth Sci., 418, 91-94.

Semmler, T., S. Varghese, R. McGrath, P. Nolan, S. L. Wang, P., and C. O'Dowd, 2008: Regional climate model simulations of NorthAtlantic cyclones: Frequency and intensity changes. Clim. Res, 36, 1-16.

Seneviratne, S. I., 2012: Historical drought trends revisited. Nature, 491, 338-339.

Seneviratne, S. I., et al., 2010: Investigating soil moisture-climate interactions in a changing climate: A review. Earth Sci. Rev., 99, 125-161. 
Seneviratne, S. I., et al., 2012: Changes in climate extremes and their impacts on the natural physical environment. In: Managing the Risks of Extreme Events and Disasters to Advance Climate Change Adaptation. A Special Report of Working Groups I and II of the Intergovernmental Panel on Climate Change (IPCC) [C. B. Field et al. (eds.)]. Cambridge University Press, Cambridge, United Kingdom, and New York, NY, USA, pp. 109-230.

Serreze, M. C., and J. A. Francis, 2006: The arctic amplification debate. Clim. Change, $76,241-264$

Serreze, M. C., M. M. Holland, and J. Stroeve, 2007: Perspectives on the Arctic's shrinking sea-ice cover. Science, 315, 1533-1536.

Serreze, M. C., A. P. Barrett, J. C. Stroeve, D. N. Kindig, and M. M. Holland, 2009: The emergence of surface-based Arctic amplification. Cryosphere, 3, 9.

Sexton, D. M. H., J. M. Murphy, M. Collins, and M. J. Webb, 2012: Multivariate probabilistic projections using imperfect climate models part I: Outline of methodology. Clim. Dyn., 38, 2513-2542.

Sheffield, J., E. F. Wood, and M. Roderick, 2012: Little change in global drought over the past 60 years Nature, 491, 435-438.

Shindell, D., and G. Faluvegi, 2009: Climate response to regional radiative forcing during the twentieth century. Nature Geosci., 2, 294-300.

Shindell, D., D. Rind, N. Balachandran, J. Lean, and J. Lonergan, 1999: Solar cycle variability, ozone, and climate. Science, 284, 305-308.

Shindell, D. T., G. A. Schmidt, R. L. Miller, and D. Rind, 2001: Northern Hemisphere winter climate response to greenhouse gas, ozone, solar, and volcanic forcing. J. Geophys. Res. Atmos., 106, 7193-7210.

Shine, K. P., J. S. Fuglestvedt, K. Hailemariam, and N. Stuber, 2005: Alternatives to the global warming potential for comparing climate impacts of emissions of greenhouse gases. Clim. Change, 68, 281-302.

Shiogama, H., T. Nagashima, T. Yokohata, S. A. Crooks, and T. Nozawa, 2006: Influence of volcanic activity and changes in solar irradiance on surface air temperatures in the early twentieth century. Geophys. Res. Lett., 33, L09702.

Shiogama, H., D. A. Stone, T. Nagashima, T. Nozawa, and S. Emori, 2012: On the linear additivity of climate forcing-response relationships at global and continental scales. Int. J. Climatol., doi:10.1002/joc.3607.

Sigmond, M., and J. C. Fyfe, 2010: Has the ozone hole contributed to increased Antarctic sea ice extent? Geophys. Rese. Lett., 37, L18502.

Sigmond, M., M. C. Reader, J. C. Fyfe, and N. P. Gillett, 2011: Drivers of past and future Southern Ocean change: Stratospheric ozone versus greenhouse gas impacts. Geophys. Res. Lett., 38, L12601.

Simmons, A. J., K. M. Willett, P. D. Jones, P. W. Thorne, and D. P. Dee, 2010: Lowfrequency variations in surface atmospheric humidity, temperature, and precipitation: Inferences from reanalyses and monthly gridded observational data sets. J. Geophys. Res. Atmos., 115, D01110

Skeie, R. B., T. K. Berntsen, G. Myhre, K. Tanaka, M. M. Kvalevåg, and C. R. Hoyle, 2011: Anthropogenic radiative forcing time series from pre-industrial times until 2010. Atmos. Chem. Phys., 11, 11827-11857.

Smirnov, D. A., and I. I. Mokhov, 2009: From Granger causality to long-term causality: Application to climatic data. Phys. Rev. E, 80, 016208.

Sokolov, A., C. Forest, and P. Stone, 2010: Sensitivity of climate change projections to uncertainties in the estimates of observed changes in deep-ocean heat content. Clim. Dyn., 34, 735-745.

Solomon, S., P. J. Young, and B. Hassler, 2012: Uncertainties in the evolution of stratospheric ozone and implications for recent temperature changes in the tropical lower stratosphere. Geophys. Res. Lett., 39, L17706.

Solomon, S., et al., 2007: Technical Summary. In: Climate Change 2007: The Physical Science Basis. Contribution of Working Group I to the Fourth Assessment Report of the Intergovernmental Panel on Climate Change [Solomon, S., D. Qin, M. Manning, Z. Chen, M. Marquis, K. B. Averyt, M. Tignor and H. L. Miller (eds.)] Cambridge University Press, Cambridge, United Kingdom and New York, NY, USA, pp. 19-92.

Son, S. W., N. F. Tandon, L. M. Polvani, and D. W. Waugh, 2009: Ozone hole and Southern Hemisphere climate change. Geophys. Res. Lett., 36, L15705.

Son, S. W., et al., 2008: The impact of stratospheric ozone recovery on the Southern Hemisphere westerly jet. Science, 320, 1486-1489.

Son, S. W., et al., 2010: Impact of stratospheric ozone on Southern Hemisphere circulation change: A multimodel assessment. J. Geophys. Res. Atmos., 115, D00M07.
SPARC CCMVal, 2010: SPARC Report on the Evaluation of Chemistry-Climate Models. SPARC Report No. 5, WCRP-132, WMO/TD-No. 1526, [V. Eyring, T. G. Shepherd and D. W. Waugh (eds.)]. Stratospheric Processes And their Role in Climate. Available at: http://www.atmosp.physics.utoronto.ca/SPARC.

St Jacques, J. M., D. J. Sauchyn, and Y. Zhao, 2010: Northern Rocky Mountain streamflow records: Global warming trends, human impacts or natural variability? Geophys. Res. Lett., 37, L06407.

Stahl, K., et al., 2010: Streamflow trends in Europe: Evidence from a dataset of nearnatural catchments. Hydrol. Earth Syst. Sci., 14, 2367-2382.

Staten, P. W., J. J. Rutz, T. Reichler, and J. Lu, 2012: Breaking down the tropospheric circulation response by forcing. Clim. Dyn., 39 2361-2375.

Steig, E. J., and A. J. Orsi, 2013: The heat is on in Antarctica. Nature Geosci., 6 87-88.

Stenchikov, G., T. L. Delworth, V. Ramaswamy, R. J. Stouffer, A. Wittenberg, and F. Zeng, 2009: Volcanic signals in oceans. J. Geophys. Res. Atmos., 114, D16104.

Stephens, G. L., and Y. X. Hu, 2010: Are climate-related changes to the character of global-mean precipitation predictable? Environ. Res. Lett., 5, 025209.

Stephens, G. L., et al., 2010: Dreary state of precipitation in global models. J. Geophys. Res. Atmos., 115, D24211.

Stern, D. I., 2006: An atmosphere-ocean time series model of global climate change. Comput. Stat. Data Anal., 51, 1330-1346.

Stone, D. A., and M. R. Allen, 2005: Attribution of global surface warming without dynamical models. Geophys. Res. Lett., 32, L18711.

Stott, P. A., and J. Kettleborough, 2002: Origins and estimates of uncertainty in predictions of twenty-first century temperature rise Nature, 416, 723-726.

Stott, P. A., and C. E. Forest, 2007: Ensemble climate predictions using climate models and observational constraints. Philos. Trans. R. Soc. A, 365, 2029-2052.

Stott, P. A., and G. S. Jones, 2009: Variability of high latitude amplification of anthropogenic warming. Geophys. Res. Lett., 36, L10701.

Stott, P. A., and G. S. Jones, 2012: Observed 21st century temperatures further constrain decadal predictions of future warming. Atmos. Sci. Lett., 13, 151-156.

Stott, P. A., D. A. Stone, and M. R. Allen, 2004: Human contribution to the European heatwave of 2003. Nature, 432, 610-614.

Stott, P. A., R. T. Sutton, and D. M. Smith, 2008a: Detection and attribution of Atlantic salinity changes. Geophys. Res. Lett., 35, L21702.

Stott, P. A., C. Huntingford, C. D. Jones, and J. A. Kettleborough, 2008b: Observed climate change constrains the likelihood of extreme future global warming. Tellus $B, 60,76-81$.

Stott, P. A., G. S. Jones, N. Christidis, F. W. Zwiers, G. Hegerl, and H. Shiogama, 2011: Single-step attribution of increasing frequencies of very warm regional temperatures to human influence. Atmos. Sci. Lett., 12, 220-227.

Stott, P. A., J. F. B. Mitchell, M. R. Allen, T. L. Delworth, J. M. Gregory, G. A. Meehl, and B. D. Santer, 2006: Observational constraints on past attributable warming and predictions of future global warming. J. Clim., 19, 3055-3069.

Stott, P. A., N. P. Gillett, G. C. Hegerl, D. J. Karoly, D. A. Stone, X. Zhang, and F. Zwiers, 2010: Detection and attribution of climate change: A regional perspective. WIREs Clim. Change, 1, 192-211.

Stott, P. A., et al., 2013: Attribution of weather and climate-related events. In: Climate Science for Serving Society: Research, Modelling and Prediction Priorities [G. R. Asrar and J. W. Hurrell (eds.)]. Springer Science+Business Media, Dordrecht, Netherlands, $477 \mathrm{pp}$.

Stramma, L., S. Schmidtko, L. Levin, and G. Johnson, 2010: Ocean oxygen minima expansions and their biological impacts. Deep-Sea Res. Pt. I, 57, 587-595.

Stroeve, J., et al., 2008: Arctic Sea ice extent plumments in 2007. Eos Trans. Am. Geophys. Union, 89, 13-14.

Stroeve, J. C., M. C. Serreze, M. M. Holland, J. E. Kay, W. Meier, and A. P. Barrett, 2012a: The Arctic's rapidly shrinking sea ice cover: A research synthesis. Clim. Change, 110 1005-1027.

Stroeve, J. C., V. Kattsov, A. Barrett, M. Serreze, T. Pavlova, M. Holland, and W. N. Meier, 2012b: Trends in Arctic sea ice extent from CMIP5, CMIP3 and observations. Geophys. Res. Lett., 39, L16502.

Swanson, K. L., G. Sugihara, and A. A. Tsonis, 2009: Long-term natural variability and 20th century climate change. Proc. Natl. Acad. Sci. U.S.A., 106, 16120-16123.

Swart, N. C., and J. C. Fyfe, 2012: Observed and simulated changes in the Southern Hemisphere surface westerly wind-stress. Geophys. Res. Lett., 39, L16711.

Tanaka, K., T. Raddatz, B. C. O'Neill, and C. H. Reick, 2009: Insufficient forcing uncertainty underestimates the risk of high climate sensitivity. Geophys. Res. Lett., 36 L16709. 
Tapiador, F. J., 2010: A joint estimate of the precipitation climate signal in Europe using eight regional models and five observational datasets. J. Clim., 23, 17191738.

Taylor, K. E., R. J. Stouffer, and G. A. Meehl, 2012: An overview of CMIP5 and the experiment design. Bull. Am. Meteorol. Soc., 93, 485-498.

Tedesco, M., J. E. Box, J. Cappellen, T. Mote, R. S. W. van der Wal, and J. Wahr, 2012: Greenland ice sheet. In State of the Climate in 2011. Bull. Am. Meteorol. Soc., 93, S150-S153.

Terray, L., 2012: Evidence for multiple drivers of North Atlantic multi-decadal climate variability. Geophys. Res. Lett., 39, L19712.

Terray, L., L. Corre, S. Cravatte, T. Delcroix, G. Reverdin, and A. Ribes, 2012: NearSurface salinity as nature's rain gauge to detect human influence on the tropical water cycle. J. Clim., 25, 958-977.

Tett, S. F. B., et al., 2007: The impact of natural and anthropogenic forcings on climate and hydrology since 1550. Clim. Dyn., 28, 3-34.

Thompson, D. W. J., and S. Solomon, 2002: Interpretation of recent Southern Hemisphere climate change. Science, 296, 895-899.

Thompson, D. W. J., and S. Solomon, 2009: Understanding recent stratospheric climate change. J. Clim., 22, 1934-1943.

Thompson, D. W. J., J. M. Wallace, P. D. Jones, and J. J. Kennedy, 2009: Identifying signatures of natural climate variability in time series of global-mean surface temperature: Methodology and insights. J. Clim., 22, 6120-6141.

Thorne, P. W., and R. S. Vose, 2010: Reanalyses suitable for characterizing long-term trends Bull. Am. Meteorol. Soc., 91, 353-361.

Thorne, P. W., et al., 2011: A quantification of uncertainties in historical tropical tropospheric temperature trends from radiosondes. J. Geophys. Res. Atmos., 116, D12116.

Timmermann, A., S. McGregor, and F. F. Jin, 2010: Wind effects on past and future regional sea level trends in the southern Indo-Pacific. J. Clim., 23, 4429-4437.

Timmreck, C., S. J. Lorenz, T. J. Crowley, S. Kinne, T. J. Raddatz, M. A. Thomas, and J. H. Jungclaus, 2009: Limited temperature response to the very large AD 1258 volcanic eruption. Geophys. Res. Lett., 36, L21708.

Ting, M. F., Y. Kushnir, R. Seager, and C. H. Li, 2009: Forced and internal twentiethcentury sst trends in the North Atlantic. J. Clim., 22 1469-1481.

Tomassini, L., P. Reichert, R. Knutti, T. F. Stocker, and M. E. Borsuk, 2007: Robust bayesian uncertainty analysis of climate system properties using Markov chain Monte Carlo methods. J. Clim., 20, 1239-1254.

Trenberth, K., 2011a: Attribution of climate variations and trends to human influences and natural variability. WIREs Clim. Change, 2, 925-930.

Trenberth, K., $2011 \mathrm{~b}$ : Changes in precipitation with climate change. Clim. Research, 47, 123-138.

Trenberth, K. E., and D. J. Shea, 2006: Atlantic hurricanes and natural variability in 2005. Geophys. Res. Lett., 33, L12704.

Trenberth, K. E., and J. T. Fasullo, 2012: Climate extremes and climate change: The Russian heat wave and other climate extremes of 2010. J. Geophys. Res. Atmos., 117, D17103.

Trenberth, K. E., J. T. Fasullo, C. O'Dell, and T. Wong, 2010: Relationships between tropical sea surface temperature and top-of-atmosphere radiation. Geophys. Res. Lett., 37, L03702.

Tung, K.-K., and J. Zhou, 2010: The Pacific's response to surface heating in $130 \mathrm{yr}$ of SST: La Niña-like or El Niño-like? J. Atmos. Sci., 67, 2649-2657.

Tung, K.-K., and J. Zhou, 2013: Using data to attribute episodes of warming and cooling in instrumental records. Proc. Natl. Acad. Sci. U.S.A., 110 2058-2063.

Tung, K. K., J. S. Zhou, and C. D. Camp, 2008: Constraining model transient climate response using independent observations of solar-cycle forcing and response. Geophys. Res. Lett., 35 L17707.

Turner, J., T. J. Bracegirdle, T. Phillips, G. J. Marshall, and J. S. Hosking, 2013: An initial assessment of Antarctic sea ice extent in the CMIP5 models. J. Clim., 26, 14731484.

Turner, J., et al., 2005: Antarctic change during the last 50 years. Int. J. Climatol., 25, $1147-1148$.

Turner, J., et al., 2009: Non-annular atmospheric circulation change induced by stratospheric ozone depletion and its role in the recent increase of Antarctic sea ice extent. Geophys. Res. Lett., 36, L08502.

Ulbrich, U., G. C. Leckebusch, and J. G. Pinto, 2009: Extra-tropical cyclones in the present and future climate: A review. Theor. Appl. Climatol., 96, 117-131.

Urban, N. M., and K. Keller, 2009: Complementary observational constraints on climate sensitivity. Geophys. Res. Lett., 36, L04708. van der Schrier, G., P. D. Jones, and K. R. Briff, 2011: The sensitivity of the PDSI to the Thornthwaite and Penman-Monteith parameterizations for potential evapotranspiration. J. Geophys. Res. Atmos., 116, D03106.

van Loon, H., and G. A. Meehl, 2008: The response in the Pacific to the sun's decadal peaks and contrasts to cold events in the Southern Oscillation. J. Atmos. Sol. Terres. Phys., 70 1046-1055.

van Loon, H., and G. A. Meehl, 2012: The Indian summer monsoon during peaks in the 11 year sunspot cycle. Geophys. Res. Lett., 39 L13701.

van Loon, H., G. A. Meehl, and D. J. Shea, 2007: Coupled air-sea response to solar forcing in the Pacific region during northern winter. J. Geophys. Res. Atmos., 112, D02108.

van Oldenborgh, G. J., A. van Urk, and M. Allen, 2012: The absence of a role of climate change in the 2011 Thailand floods. Bull. Am. Meteorol. Soc., 93, 1047-1049.

van Oldenborgh, G. J., F. J. Doblas Reyes, S. S. Drijfhout, and E. Hawkins, 2013: Reliability of regional climate model trends. Environ. Res. Lett., 8, 014055.

Vecchi, G. A., and B. J. Soden, 2007: Global warming and the weakening of the tropical circulation. J. Clim., 20, 4316-4340.

Vecchi, G. A., K. L. Swanson, and B. J. Soden, 2008: Whither hurricane activity. Science, 322, 687-689

Veryard, H. G., 1963: A review of studies on climate fluctuations during the period of the meteorological. Changes of Climate: Proceedings of the Rome Symposium Organised by UNESCO and WMO, pp. 3-15.

Villarini, G., and G. A. Vecchi, 2012: Twenty-first-century projections of North Atlantic tropical storms from CMIP5 models. Nature Clim. Change, 2, 604-607.

Villarini, G., and G. A. Vecchi, 2013: Projected increases in North Atlantic tropical cyclone intensity from CMIP5 models. J. Clim., 26, 3231-3240.

Visser, H., and A. C. Petersen, 2012: Inference on weather extremes and weather related disasters: A review of statistical methods. Clim. Past, 8 265-286.

von Schuckmann, K., F. Gaillard, and P. Y. Le Traon, 2009: Global hydrographic variability patterns during 2003-2008. J. Geophys. Res.J. Geophys. Res. Oceans, 114, C09007.

Vorosmarty, C., L. Hinzman, and J. Pundsack, 2008: Introduction to special section on changes in the arctic freshwater system: Identification, attribution, and impacts at local and global scales. J. Geophys. Res. Biogeosci., 113, G01591.

Vuille, M., G. Kaser, and I. Juen, 2008: Glacier mass balance variability in the Cordillera Blanca, Peru and its relationship with climate and the large-scale circulation. Global Planet. Change, 62, 14-28.

Walker, R. T., T. K. Dupont, D. M. Holland, B. R. Parizek, and R. B. Alley, 2009: Initial effects of oceanic warming on a coupled ocean-ice shelf-ice stream system. Earth Planet. Sci. Lett., 287, 483-487.

Wan, H., X. Zhang, F. W. Zwiers, and H. Shiogama, 2013: Effect of data coverage on the estimation of mean and variability of precipitation at global and regional scales. J. Geophys. Res. Atmos., 118, 534-546.

Wang, D. B., and M. Hejazi, 2011: Quantifying the relative contribution of the climate and direct human impacts on mean annual streamflow in the contiguous United States. Water Resour. Res., 47, W00J12.

Wang, J., and X. Zhang, 2008: Downscaling and projection of winter extreme daily precipitation over North America. J. Clim., 21, 923-937.

Wang, J., et al., 2009a: Is the Dipole Anomaly a major driver to record lows in Arctic summer sea ice extent? Geophys. Res. Lett., 36, L05706.

Wang, M., and J. E. Overland, 2012: A sea ice free summer Arctic within 30 years: An update from CMIP5 models. Geophys. Res. Lett., 39, L18501.

Wang, M. Y., and J. E. Overland, 2009: A sea ice free summer Arctic within 30 years? Geophys. Res. Lett., 36, L07502.

Wang, M. Y., J. E. Overland, V. Kattsov, J. E. Walsh, X. D. Zhang, and T. Pavlova, 2007: Intrinsic versus forced variation in coupled climate model simulations over the Arctic during the twentieth century. J. Clim., 20 1093-1107.

Wang, X. L., V. R. Swail, F. W. Zwiers, X. Zhang, and Y. Feng, 2009b: Detection of external influence on trends of atmospheric storminess and northern oceans wave heights. Clim. Dyn., 32, 189-203.

Wassmann, P., C. M. Duarte, S. Agusti, and M. K. Sejr, 2011: Footprints of climate change in the Arctic marine ecosystem. Global Change Biol., 17, 1235-1249.

Wen, Q. H., X. Zhang, Y. Xu, and B. Wang, 2013: Detecting human influence on extreme temperatures in China. Geophys. Res. Lett., 40, 1171-1176.

Wentz, F. J., L. Ricciardulli, K. Hilburn, and C. Mears, 2007: How much more rain will global warming bring? Science, 317 233-235.

Westra, S., L. V. Alexander, and F. W. Zwiers, 2013: Global increasing trends in annual maximum daily precipitation. J. Clim., doi:10.1175/JCLI-D-12-00502.1. 
White, W. B., and Z. Y. Liu, 2008: Non-linear alignment of El Nino to the 11-yr solar cycle. Geophys. Res. Lett., 35, L19607.

Wigley, T. M. L., and B. D. Santer, 2013: A probabilistic quantification of the anthropogenic component of twentieth century global warming. Clim. Dyn., 40, 1087-1102.

Wigley, T. M. L., C. M. Ammann, B. D. Santer, and K. E. Taylor, 2005: Comment on "Climate forcing by the volcanic eruption of Mount Pinatubo" by David $\mathrm{H}$. Douglass and Robert S. Knox. Geophys. Res. Lett., 32, L20709.

Wijffels, S., et al., 2008: Changing expendable bathythermograph fall rates and their impact on estimates of thermosteric sea level rise. J. Clim., 21, 5657-5672.

Wilcox, L. J., B. J. Hoskins, and K. P. Shine, 2012: A global blended tropopause based on ERA data. Part II: Trends and tropical broadening. Q. J. R. Meteorol. Soc., 138, 576-584.

Willett, K. M., N. P. Gillett, P. D. Jones, and P. W. Thorne, 2007: Attribution of observed surface humidity changes to human influence. Nature, 449, 710-712

Willett, K. M., P. D. Jones, N. P. Gillett, and P. W. Thorne, 2008: Recent changes in surface humidity: Development of the HadCRUH dataset. J. Clim., 21, 53645383.

Wilson, D., H. Hisdal, and D. Lawrence, 2010: Has streamflow changed in the Nordic countries? Recent trends and comparisons to hydrological projections. J. Hydrol., 394, 334-346.

WMO (World Meteorological Organization), 2011: Scientific Assessment of Ozone Depletion: 2010. Global Ozone Research and Monitoring Project-Report No. 52, World Meterological Organization, Geneva, Switzerland, $516 \mathrm{pp}$.

Wong, A. P. S., N. L. Bindoff, and J. A. Church, 1999: Large-scale freshening of intermediate waters in the Pacific and Indian oceans. Nature, 400, 440-443.

Wood, K. R., and J. E. Overland, 2010: Early 20th century Arctic warming in retrospect. Int. J. Climatol, 30, 1269-1279.

Woollings, T., 2008: Vertical structure of anthropogenic zonal-mean atmospheric circulation change. Geophys. Res. Lett., 35, L19702.

Woollings, T., M. Lockwood, G. Masato, C. Bell, and L. J. Gray, 2010: Enhanced signatures of solar variability in Eurasian winter climate. Geophys. Res. Lett., 37 L20805.

Wu, Q. G., and D. J. Karoly, 2007: Implications of changes in the atmospheric circulation on the detection of regional surface air temperature trends. Geophys. Res. Lett., 34, L08703.

Wu, Z. H., N. E. Huang, J. M. Wallace, B. V. Smoliak, and X. Y. Chen, 2011: On the time-varying trend in global-mean surface temperature. Clim. Dyn., 37 759-773.

Xie, S.-P., C. Deser, G. A. Vecchi, J. Ma, H. Teng, and A. T. Wittenberg, 2010: Global warming pattern formation: Sea surface temperature and rainfall. J. Clim., 23, 966-986.

Yamaguchi, S., R. Naruse, and T. Shiraiwa, 2008: Climate reconstruction since the Little Ice Age by modelling Koryto glacier, Kamchatka Peninsula, Russia. J. Glaciol., 54, 125-130.

Yang, X., J. C. Fyfe, and G. M. Flato, 2010: The role of poleward energy transport in Arctic temperature. Geophys. Res. Lett., 37, L14803.

Yiou, P., R. Vautard, P. Naveau, and C. Cassou, 2007: Inconsistency between atmospheric dynamics and temperatures during the exceptional 2006/2007 fall/ winter and recent warming in Europe. Geophys. Res. Lett., 34, L21808.

Yoshimori, M., and A. J. Broccoli, 2008: Equilibrium response of an atmospheremixed layer ocean model to different radiative forcing agents: Global and zonal mean response. J. Clim., 21, 4399-4423.

Young, P. J., et al., 2012: Agreement in late twentieth century Southern Hemisphere stratospheric temperature trends in observations and CCMVal-2, CMIP3 and CMIP5 models. J. Geophys. Res. Atmos., 118 605-613.

Zhang, J., R. Lindsay, A. Schweiger, and M. Steele, 2013: The impact of an intense summer cyclone on 2012 Arctic sea ice retreat. Geophys. Res. Lett., 40, 720-726.

Zhang, J. L., 2007: Increasing Antarctic sea ice under warming atmospheric and oceanic conditions. J. Clim., 20, 2515-2529.

Zhang, R., and T. L. Delworth, 2009: A new method for attributing climate variations over the Atlantic Hurricane Basin's main development region. Geophys. Res. Lett., 36, L06701.

Zhang, R., et al., 2012: Have aerosols caused the observed Atlantic Multidecadal Variability? J. Atmos. Sci., 70, 1135-1144.

Zhang, X. B., et al., 2007: Detection of human influence on twentieth-century precipitation trends. Nature, 448, 461-465.

Zhang, X. D., A. Sorteberg, J. Zhang, R. Gerdes, and J. C. Comiso, 2008: Recent radical shifts of atmospheric circulations and rapid changes in Arctic climate system. Geophys. Res. Lett., 35, L22701.
Zhou, J., and K.-K. Tung, 2013a: Deducing multidecadal anthropogenic global warming trends using multiple regression analysis. J. Atmos. Sci., 70, 3-8.

Zhou, J., and K.-K. Tung, 2013b: Observed tropospheric temperature response to 11-yr solar cycle and what it reveals about mechanisms. J. Atmos. Sci., 70, 9-14.

Zhou, Y., and G. Ren, 2011: Change in extreme temperature event frequency over mainland China, 1961-2008. Climate Research, 50, 125-139.

Zickfeld, K., M. Eby, H. D. Matthews, and A. J. Weaver, 2009: Setting cumulative emissions targets to reduce the risk of dangerous climate change. Proc. Natl. Acad. Sci. U.S.A., 106, 16129-16134.

Zickfeld, K., et al., 2013: Long-term climate change commitment and reversibility: An EMIC intercomparison. J. Clim., doi:10.1175/JCLI-D-12-00584.1.

Zorita, E., T. F. Stocker, and H. von Storch, 2008: How unusual is the recent series of warm years? Geophys. Res. Lett., 35, L24706.

Zunz, V., H. Goosse, and F. Massonnet, 2013: How does internal variability influence the ability of CMIP5 models to reproduce the recent trend in Southern Ocean sea ice extent? Cryosphere, 7, 451-468.

Zwiers, F. W., X. Zhang, and Y. Feng, 2011: Anthropogenic influence on long return period daily temperature extremes at regional scales. J. Clim., 24, 881-892. 